\title{
TANIA MARIE OGAWA ABE
}

Internação e mortalidade por doença cardiovascular e cerebrovascular no período anterior e posterior à lei antitabaco na cidade de São Paulo

Tese apresentada à Faculdade de Medicina da Universidade de São Paulo, para obtenção do título de Doutor em Ciências

Programa de Cardiologia Orientadora: Dra. Jaqueline Ribeiro Scholz

São Paulo

2016 
Dados Internacionais de Catalogação na Publicação (CIP)

Preparada pela Biblioteca da

Faculdade de Medicina da Universidade de São Paulo

Creprodução autorizada pelo autor

Abe, Tania Marie Ogawa

Internação e mortalidade por doença cardiovascular e cerebrovascular no período anterior e posterior à lei antitabaco na cidade de São Paulo / Tania Marie Ogawa Abe. -- São Paulo, 2016.

Tese(doutorado)--Faculdade de Medicina da Universidade de São Paulo. Programa de Cardiologia.

Orientador: Jaqueline Ribeiro Scholz.

Descritores: 1.Leis 2.Política antifumo 3.Infarto do miocárdio 4.Acidente vascular cerebral 5.Mortalidade 6.Admissão do paciente 7.Meio ambiente

USP/FM/DBD-296/16 


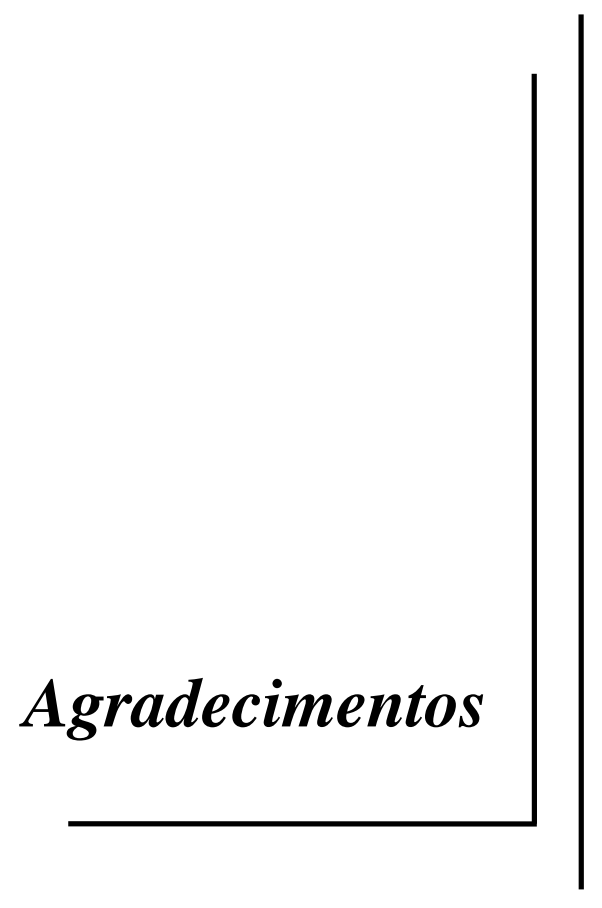


À minha orientadora, Dra. Jaqueline Ribeiro Scholz, por sua crença inabalável no projeto de pesquisa e por sempre me incentivar e ajudar durante todas as etapas da pesquisa.

Ao Dr. Moacyr Roberto Cuce Nobre, médico pesquisador da Unidade de Epidemiologia Clínica do InCor, por sua contribuição na geração de idéias e questionamentos que deveriam ser respondidos durante esta pesquisa e pela análise crítica da metodologia utilizada.

Ao Dr. Eduardo de Masi, biólogo da Coordenadoria Regional de Saúde Sul da Secretaria Municipal de Saúde de São Paulo, por sua contribuição no ajuste da metodologia e na análise estatística dos dados e na elaboração do artigo científico.

À Dra. Fabiana Goulart Marcondes Braga, médica assistente do Núcleo de Transplante Cardíaco do InCor, por sua análise crítica do documento da tese e valiosas sugestões em diferentes etapas da produção da tese.

Ao Dr. Ubiratan de Paula Santos, médico assitente da Divisão de Pneumologia do InCor, por sua análise crítica do documento de tese.

Ao Prof. Dr. Alfésio Luís Ferreira Braga, professor da UNICID e da Universidade Católica de Santos, por sua análise crítica do documento da tese, em especial à análise estatística.

Ao Dr. Márcio Sommer Bittencourt, médico assistente do Hospital Universitário e médico pesquisador vinculado à Universidade de Harvard, por sua análise crítica do documento da tese, principalmente à análise estatística.

Aos membros participantes da minha banca de qualificação, Prof. Dr. Whady Armindo Hueb, Profa. Dra. Márcia Furquim de Almeida e Dr. Alexandre da 
Costa Pereira, pelas valiosas sugestões que contribuíram para o aprimoramento desta tese.

Ao Dr. Euro Couto Carros, por sua contribuição na análise inicial dos dados.

Ao Prof. Dr. Antonio Carlos Pereira Barretto, diretor da Unidade de Prevenção e Reabilitação do InCor, que permitiu e colaborou com a realização do estudo.

Ao Sr. José Dínio Vaz Mendes, Sra. Vera Lucia Rodrigues Lopes Osiano, Sra. Mônica Aparecida Marcondes Cecilio, assistentes técnicos da Coordenadoria de Planejamento de Saúde da Secretaria Estadual de Saúde de São Paulo, pelo auxílio na aquisição dos dados.

À Sra. Maria Cristina Megid, médica diretora do Centro de Vigilância Sanitária da Secretaria Estadual de Saúde, pelo auxílio na aquisição dos dados.

À Sra. Maria Helena R. B. Dias, química, gerente da Divisão de Tecnologia de Avaliação da Qualidade do Ar da CETESB, pelo fornecimento dos dados de poluentes, temperatura e umidade do ar.

Aos hospitais Beneficência Portuguesa de São Paulo, Hospital Cruz Azul, Hospital do Coração, Hospital e Maternidade Dom Antônio de Alvarenga, Hospital e Maternidade São Camilo Ipiranga, Hospital e Maternidade São Camilo Pompéia, Hospital e Maternidade São Camilo Santana, Hospital Israelita Albert Einstein, Hospital Nove de Julho, Hospital Paulistano, Hospital Samaritano, Hospital Total Cor, Hospital Santa Cecília, Hospital Santa Cruz, Hospital Sirio Libanês, Instituto do Coração, pelo auxílio no fornecimento de dados.

À Comissão de Pós-graduação, em especial à Sra. Neusa Rodrigues Dini, Sra. Juliana Lattari Sobrinho e Sra. Monica Souto, pela atenção e orientação. 


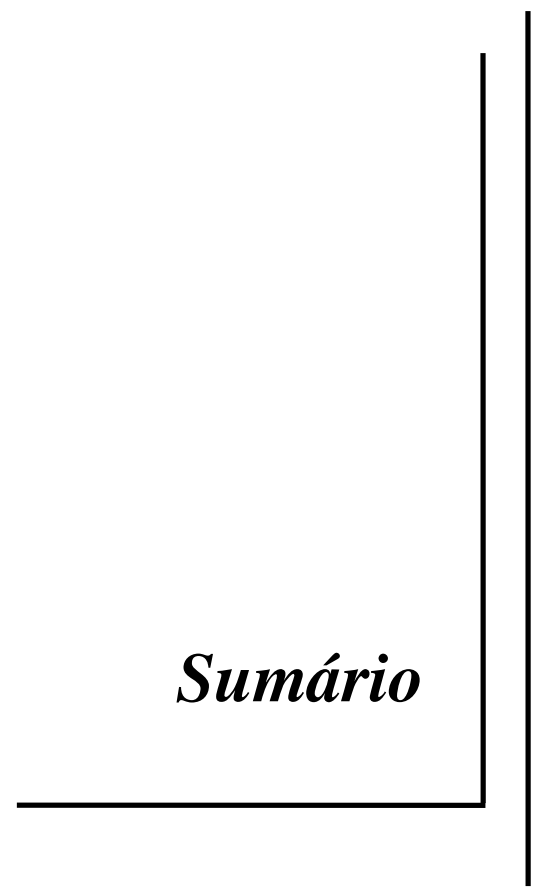




\section{SUMÁRIO}

LISTA DE ABREVIATURAS, SIGLAS E SÍMBOLOS

LISTA DE TABELAS

LISTA DE FIGURAS

RESUMO

\section{ABSTRACT}

1 INTRODUÇÃO

1.1 Tabagismo e doença cardiovascular ……………………………………... 2

1.2 Tabagismo e disfunção endotelial $\quad$ ………………………………………...

1.3 Estratégias para redução dos riscos associados ao tabagismo ........ $\quad 4$

1.3.1 Convenção-Quadro para o Controle do Tabagismo ……………. 4

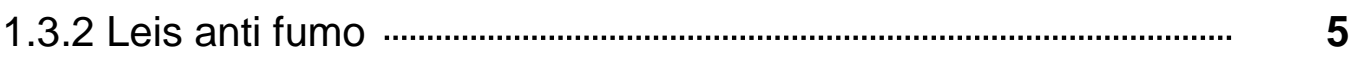

1.3.2.a História das leis anti fumo no mundo …………………………..... $\quad 6$

1.3.2.b História das leis anti fumo no Brasil ............................................. $\quad 8$

1.3.2.c A lei anti fumo no estado de São Paulo ………………………... $\quad 12$

1.4 Interferentes externos que podem influenciar na taxa de eventos

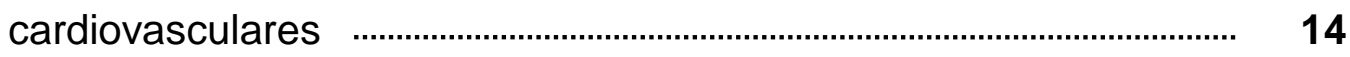

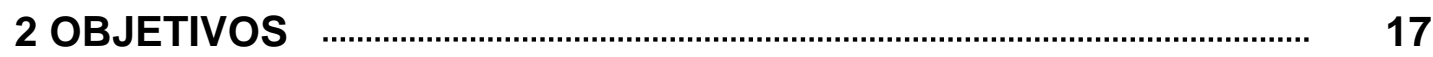

3 MATERIAIS E MÉTODOS

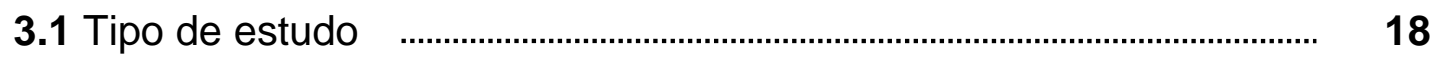

3.2 Coleta de dados $\quad$.................................................................................................... 18

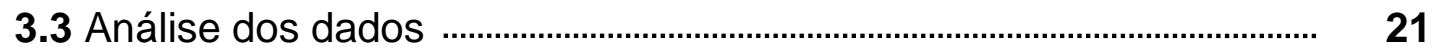

3.3.1 Modelos e programas estatísticos ………………………………..... 21

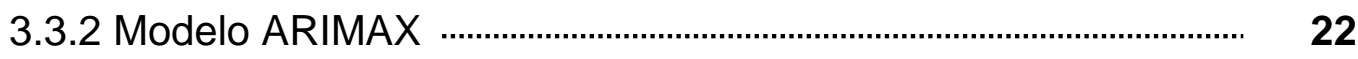

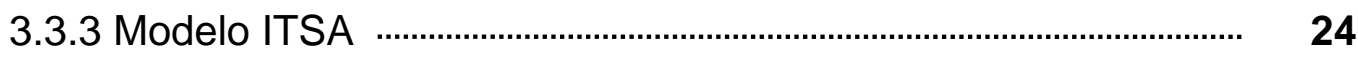

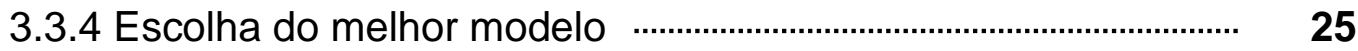

3.3.5 Diferenciação entre ARIMAX e ITSA-ARIMAX ………………….. $\quad 25$

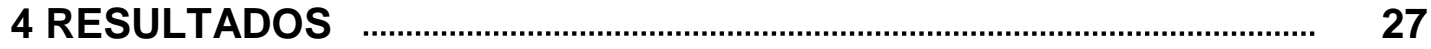

4.1 Análise da morbidade hospitalar …………………………………………. 27

4.2 Análise da mortalidade $\quad$.................................................................................. $\quad 29$

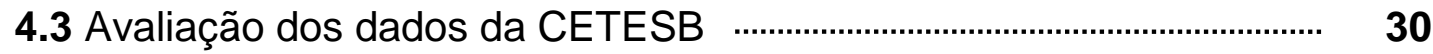

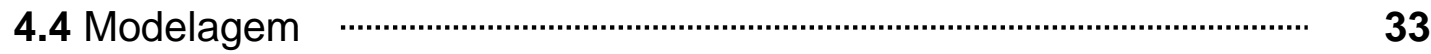


4.4.1 Modelo ARIMAX internação por "Infarto" ....................................... 33

4.4.2 Modelo ARIMAX internação por "AVC" …………………………... $\quad 34$

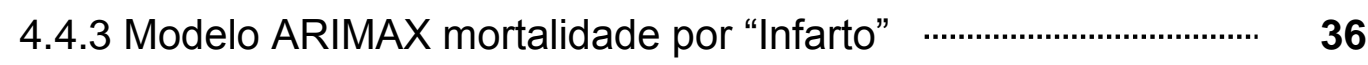

4.4.4 Modelo ARIMAX mortalidade por "AVC" ........................................ $\quad 37$

4.4.5 Modelo ITSA-ARIMAX internação por "Infarto" ............................. 38

4.4.6 Modelo ITSA-ARIMAX internação por "AVC" ................................. $\quad 39$

4.4.7 Modelo ITSA-ARIMAX mortalidade por "Infarto" ............................ $\quad 40$

4.4.8 Modelo ITSA-ARIMAX mortalidade por "AVC" ............................... 4

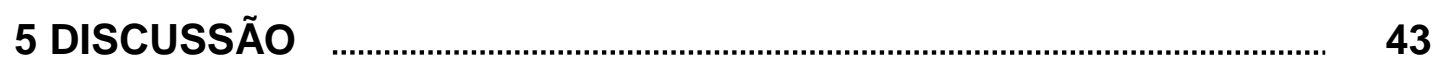

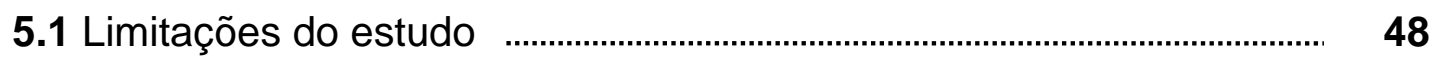

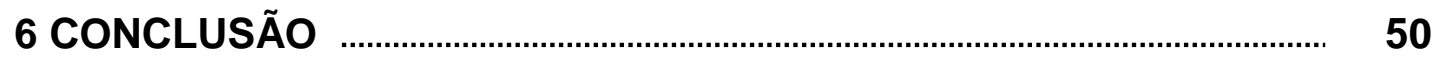

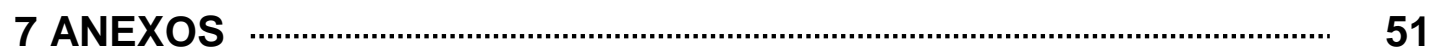

ANEXO A - Modelo estimado para taxa de internações por “Infarto", metodologia ARIMAX ………………………….. 51

ANEXO B - Modelo estimado para taxa de internações por “AVC", metodologia ARIMAX …………………………..... 54

ANEXO C - Modelo estimado para taxa de mortalidade por “Infarto", metodologia ARIMAX ………………………….. 57

ANEXO D - Modelo estimado para taxa de mortalidade por

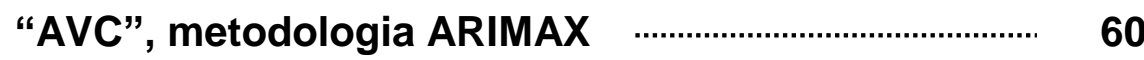

ANEXO E - Modelo estimado para taxa de internações por “Infarto”, metodologia ITSA-ARIMAX …………………... 63

ANEXO F - Modelo estimado para taxa de internações por "AVC", metodologia ITSA-ARIMAX …………………….. $\quad 70$

ANEXO G - Modelo estimado para taxa de mortalidade por “Infarto”, metodologia ITSA-ARIMAX …………………... 74

ANEXO H - Modelo estimado para taxa de mortalidade por "AVC", metodologia ITSA-ARIMAX

8 REFERÊNCIAS 


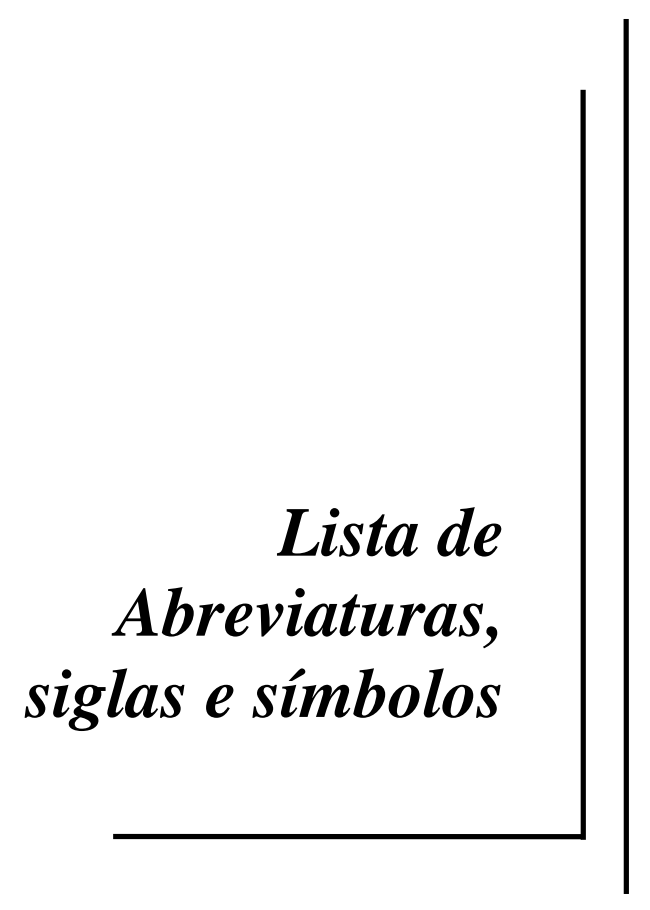




\section{LISTA DE ABREVIATURAS, SIGLAS E SÍMBOLOS (ORDEM ALFABÉTICA)}

\begin{tabular}{|c|c|}
\hline$\%$ & porcentagem \\
\hline * & multiplicação \\
\hline “AVC" & Acidente vascular cerebral (CID I63 e I64 em conjunto) \\
\hline “Infarto" & $\begin{array}{l}\text { Infarto agudo do miocárdio e síndromes correlatas (CID I21, } \\
\text { I22, I23 e I24 em conjunto) }\end{array}$ \\
\hline+ & soma \\
\hline$<$ & menor \\
\hline$=$ & igual \\
\hline$>$ & maior \\
\hline$\geq$ & maior ou igual \\
\hline$\leq$ & menor ou igual \\
\hline acf & função de autocorrelação \\
\hline aic & critério de informação de Akaike \\
\hline ANVISA & Agência Nacional de Vigilância Sanitária \\
\hline ARIMAX & $\begin{array}{l}\text { Modelo Autorregressivo, Integrado e de Médias Móveis, com } \\
\text { variáveis exógenas }\end{array}$ \\
\hline CAPPesq & Comissão de Ética para Análise de Projetos de Pesquisa \\
\hline CETESB & Companhia Ambiental do Estado de São Paulo \\
\hline CID I21 & Código para "Infarto agudo do miocárdio" \\
\hline CID I22 & Código para "Infarto do miocárdio recorrente" \\
\hline
\end{tabular}


CID I23

Código para "Complicações atuais subseqüentes ao infarto agudo do miocárdio"

CID I24

Código para "Outras doenças isquêmicas agudas do coração"

CID I63

Código para "Acidente vascular cerebral isquêmico"

CID I64

Código para "Acidente vascular cerebral, não classificado como isquêmico ou hemorrágico"

CID

Código Internacional de Doenças

$\mathrm{CO}$

monóxido de carbono

corrgram

correlograma dos resíduos

CQCT

Convenção-Quadro para o Controle do Tabaco

DATASUS Departamento de Informática do Sistema Único de Saúde

dfgls teste modificado de Dickey-Fuller

dfuller

teste aumentado de Dickey-Fuller

EQMP

erro quadrático médio de previsão

EUA

Estados Unidos da América

Fundação SEADE Fundação Sistema Estadual de Análise de Dados

HC-FMUSP

Hospital das Clínicas da Faculdade de Medicina da Universidade de São Paulo

IBGE Instituto Brasileiro de Geografia e Estatística

INCOR Instituto do Coração

ITSA Análise de séries temporais interrompida

Lag defasagem

MP10 material particulado inalável menor que 10 micrômetros 


\begin{tabular}{|c|c|}
\hline $\mathrm{n}^{0}$ & número \\
\hline NO2 & dióxido de nitrogênio \\
\hline${ }^{\circ} \mathrm{C}$ & graus Celsius \\
\hline OMS & Organização Mundial da Saúde \\
\hline OPAS & Organização Pan Americana de Saúde \\
\hline $\mathrm{p}$ & significância estatística \\
\hline pacf & função de autocorrelação parcial \\
\hline pperron & teste de Phillips-Perron \\
\hline ppm & partes por milhão \\
\hline prais & regressão de Prais-Winsten e Cochrane-Orcutt \\
\hline Pro-AIM & Programa de Aprimoramento das Informações de Mortalidade \\
\hline $\mathrm{SIH}$ & Sistema de Informações Hospitalares \\
\hline SIM & Sistema de Informação sobre Mortalidade \\
\hline SO2 & dióxido de enxofre \\
\hline SOCESP & Sociedade de Cardiologia do estado de São Paulo \\
\hline Stata & software de estatística de análise de dados® \\
\hline SUS & Sistema Único de Saúde \\
\hline swilk & teste de Shapiro-Wilk \\
\hline Tmax & temperatura máxima \\
\hline Tmin & temperatura mínima \\
\hline UR & umidade relativa do ar \\
\hline varsoc & critério de ordem de seleção autorregressiva vetorial \\
\hline xcorr & função de correlação cruzada \\
\hline
\end{tabular}


$\beta$

$\mu \mathrm{g} / \mathrm{m} 3$ erro tipo II

micrômetro por metro cúbico 


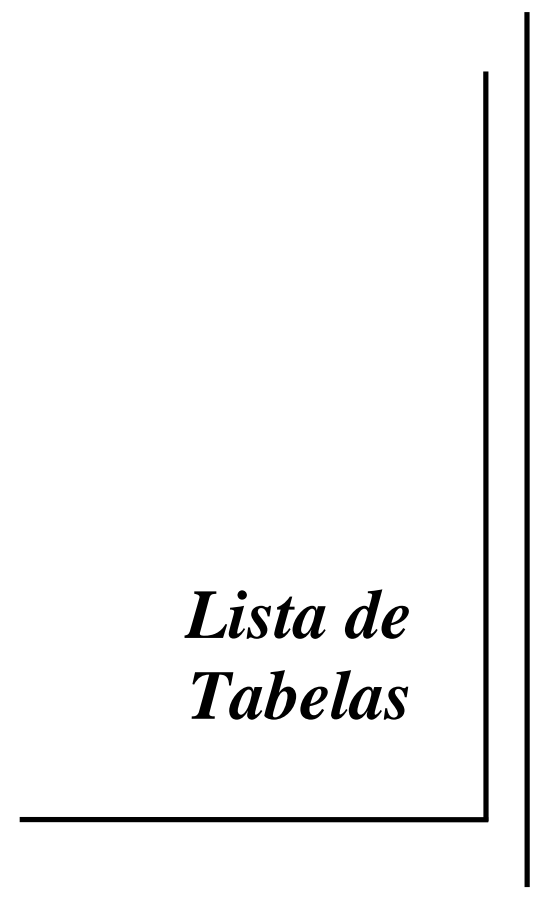




\section{LISTA DE TABELAS}

Tabela 1 - Número mensal de internações hospitalares ocorridas em hospitais do SUS com diagnóstico de "Infarto" (CIDs I21, 122, I23 e I24), Município de São Paulo, janeiro de 2005 a dezembro de $2010 \ldots \ldots \ldots \ldots \ldots \ldots \ldots \ldots \ldots . . . . . . . . .27$

Tabela 2 - Número mensal de internações hospitalares ocorridas em hospitais do SUS com diagnóstico de "AVC" (CIDs 163 e 164), Município de São Paulo, janeiro de 2005 a dezembro de 2010

Tabela 3 - Número mensal total de internações hospitalares ocorridas em hospitais do SUS, Município de São

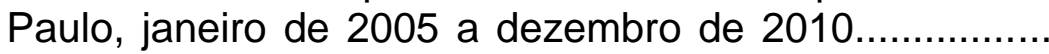

Tabela 4 - Estimativa da população SUS exclusiva do Município de São Paulo, janeiro de 2005 a dezembro de 2010.....

Tabela 5 - $\quad$ Número mensal de óbitos por "Infarto" (CIDs I21, I22, I23 e 124), Município de São Paulo, janeiro de 2005 a dezembro de 2010

Tabela 6 - $\quad$ Número mensal de óbitos por "AVC" (CIDs I63 e I64), Município de São Paulo, janeiro de 2005 a dezembro de 2010 .

Tabela 7 - Valores médios mensais da concentração de poluentes e variáveis ambientais, Município de São Paulo, janeiro de 2005 a dezembro de 2010

Tabela 8 - Percentual de tabagistas segundo a VIGITEL, Município de São Paulo, 2006 a 2011 


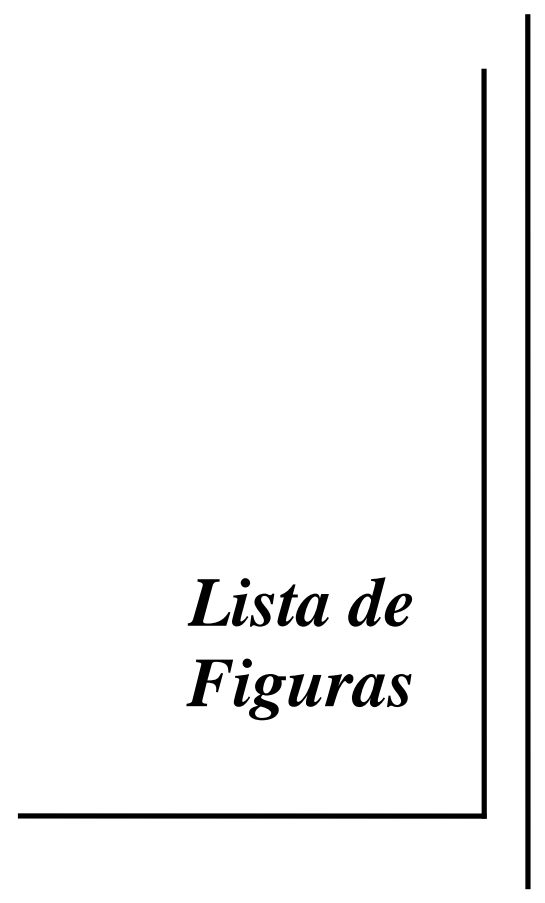




\section{LISTA DE FIGURAS}

Figura 1 - Taxa observada de internações por "Infarto" e taxa prevista de internações por "Infarto", rede hospitalar SUS, Município de São Paulo, 2005 a 2010. IC 95\%, modelo ARIMAX

Figura 2 - Taxa observada de internações por "AVC" e taxa prevista de internações por "AVC", rede hospitalar SUS, Município de São Paulo, 2005 a 2010. IC 95\%, modelo ARIMAX......

Figura 3 - Taxa observada de mortalidade por "Infarto" e taxa prevista de mortalidade por "Infarto", Município de São Paulo, 2005 a 2010. IC 95\%, modelo ARIMAX.

Figura 4 - Taxa observada de mortalidade por "AVC" e taxa prevista de mortalidade por "AVC", Município de São Paulo, 2005 a 2010. IC 95\%, modelo ARIMAX

Figura 5 - Taxa observada de internações por "Infarto" e taxa prevista de internações por "Infarto", rede hospitalar SUS, Município de São Paulo, 2005 a 2010. IC 95\%, modelo ITSA-ARIMAX

Figura 6 - Taxa observada de internações por "AVC" e taxa prevista de internações por "AVC", rede hospitalar SUS, Município de São Paulo, 2005 a 2010. IC 95\%, modelo ITSAARIMAX.

Figura 7 - Taxa observada de mortalidade por "Infarto" e taxa prevista de mortalidade por "Infarto", Município de São Paulo, 2005 a 2010. IC 95\%, modelo ITSA-ARIMAX.........

Figura 8 - Taxa observada de mortalidade por "AVC" e taxa prevista de mortalidade por "AVC", Município de São Paulo, 2005 a 2010. IC 95\%, modelo ITSA-ARIMAX 


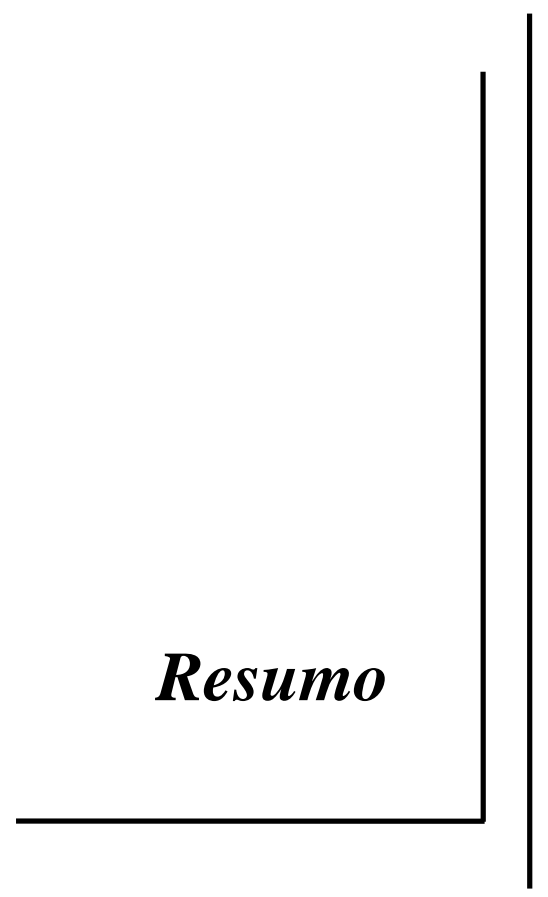


Abe TMO. Internação e mortalidade por doença cardiovascular e cerebrovascular no período anterior e posterior à lei antitabaco na cidade de São Paulo [tese]. São Paulo: Faculdade de Medicina, Universidade de São Paulo; 2016.

Introdução - As leis de restrição ao fumo têm sido amplamente difundidas pelo mundo principalmente na última década. Estudos prévios e meta análises têm demonstrado uma redução nas taxas de infarto do miocárdio após a vigência dessas leis. Entretanto, para a população latino-americana, as informações ainda são escassas. Na primeira fase deste estudo foi demonstrada a implementação bem sucedida da lei anti fumo na cidade de São Paulo, com uma redução expressiva nos níveis de monóxido de carbono em restaurantes, bares e casas noturnas. Objetivo - Avaliar se a implementação da lei anti fumo na cidade de São Paulo em 2009 foi associada a uma redução nas taxas de mortalidade e internação hospitalar por infarto do miocárdio e acidente vascular cerebral. Métodos - Foi realizado um estudo de séries temporais mensais entre janeiro de 2005 e dezembro de 2010. Os dados foram provenientes do DATASUS, o principal sistema de informação pública de saúde disponível no Brasil e do Sistema de Informação sobre Mortalidade (SIM). A análise foi realizada utilizando o método Auto-regressivo, Integrado e de Médias Móveis com variáveis exógenas (ARIMAX), modelado por variáveis ambientais e poluentes atmosféricos para avaliar as taxas de mortalidade e internação hospitalar antes da lei e prever os eventos após a lei. Também foi realizada análise utilizando o método de Análise de Séries Temporais Interrompida (ITSA), em conjunto com o ARIMAX, para 
comparar o período anterior à lei, o momento em que entrou em vigor e o período após a lei. Resultados - Foi observada uma redução nas taxas de internação hospitalar $(-5.4 \%$ nos primeiros 3 meses após a lei) e mortalidade $(-11.9 \%$ nos primeiros 17 meses após a lei) por infarto do miocárdio com o método ARIMAX e uma redução na taxa de internação hospitalar $\left(-7,4 \%\right.$ entre $07^{\circ}$ e o $12^{\circ}$ mês após a lei) e mortalidade $(-5,3 \%$ nos primeiros 17 meses após a lei) por acidente vascular cerebral, com a mesma metodologia. Conclusão - As taxas de internação hospitalar e mortalidade por infarto do miocárdio e acidente vascular cerebral foram reduzidas após o início da vigência da lei anti fumo.

Descritores: leis; política anti fumo; infarto do miocárdio; acidente vascular cerebral; mortalidade; admissão do paciente; meio ambiente. 


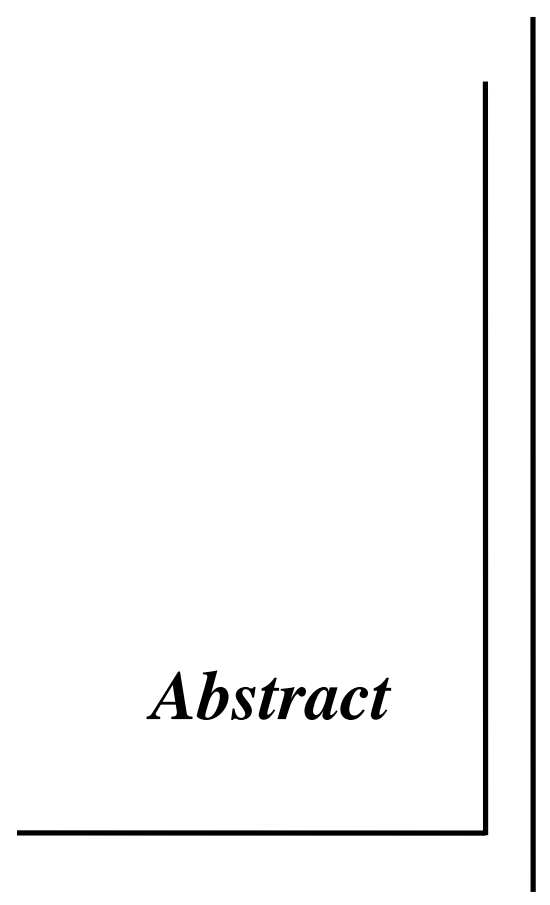


Abe TMO. Hospital admission and mortality rate for acute myocardial infarction and stroke before and after the enactment of the smoking ban law in São Paulo city [thesis]. São Paulo: "Faculdade de Medicina, Universidade de São Paulo"; 2016.

Background - Smoking restriction laws have spread worldwide during the last decade. Previous studies and meta-analyses have shown a decline in the community rates of myocardial infarction and/or heart attack after enactment of these laws. However, data are scarce about the Latin American population. In the first phase of this study, we reported the successful implementation of the law in São Paulo city, with a decrease in carbon monoxide rates in places like restaurants, bars, and nightclubs. Objective - To evaluate whether the 2009 implementation of a smoking ban law in São Paulo city was associated with a reduction in rates of mortality and hospital admissions for myocardial infarction and stroke. Methods - We performed a time series study of monthly rates of mortality and hospital admissions for acute myocardial infarction and stroke from January 2005 to December 2010. The data were derived from DATASUS, the primary public health information system available in Brazil and from Mortality Information System (SIM). Adjustments and analyses were performed using the Autoregressive Integrated Moving Average with exogenous variables (ARIMAX) method modeled by environmental variables and atmospheric pollutants to evaluate the mortality and hospital admission rate before the law and forecast events after the law. We also used Interrupted Time Series Analysis (ITSA) in conjunction with ARIMAX to compare the period before the law, the moment of 
implementation of the law, and the period after the law. Results - We observed a reduction in hospital admission rate $(-5.4 \%$ in the first 3 months after the law) and mortality rate $(-11.9 \%$ in the first 17 months after the law) for myocardial infarction with ARIMAX method. We observed a reduction in hospital admission rate $(-7,4 \%$ between the $7^{\text {th }}$ and the $12^{\text {th }}$ month after the law) and mortality rate $(-5,3 \%$ in the first 17 months after the law) for stroke with ARIMAX method. Conclusions Hospital admission rate and mortality rate for myocardial infarction and stroke were reduced after the smoking ban law was implemented.

Descriptors: laws; smoke-free policy; myocardial infarction; stroke; mortality; patient admission; environment. 


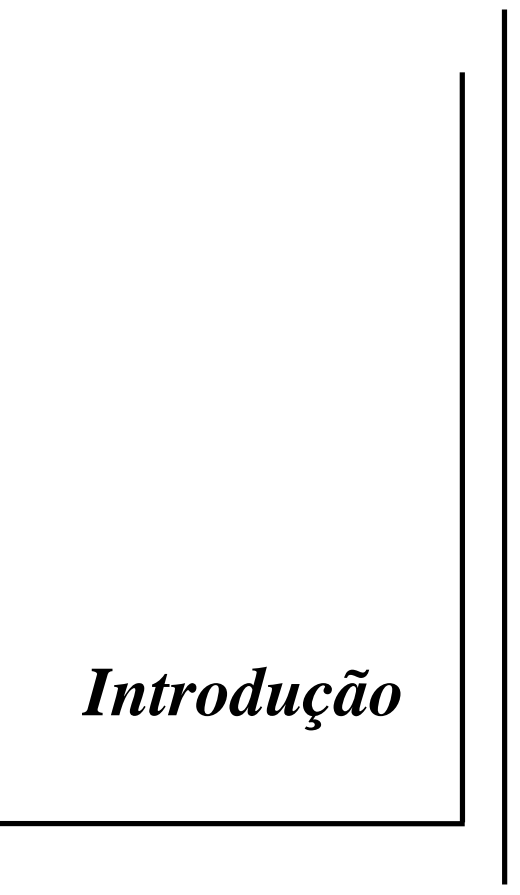


Introdução

\section{INTRODUÇÃO}

As doenças do aparelho circulatório são a principal causa de morte na maior parte dos países desenvolvidos. Contudo, nos últimos anos, tem-se observado uma tendência a declínio nas taxas de mortalidade por doenças cardiovasculares, com tendência a aproximação em relação à mortalidade por neoplasias. No Brasil, as doenças do aparelho circulatório também são a principal causa de morte, observando-se que as doenças cerebrovasculares e as doenças isquêmicas do coração são as mais prevalentes e totalizam mais de $60 \%$ dos óbitos.

Em 2011, as doenças do aparelho circulatório representaram $28,6 \%$ dos óbitos no Brasil, ${ }^{1}$ sendo que, no geral, vêm sendo observado um declínio nestas taxas ao longo do tempo. Quando se desagregam as doenças do aparelho circulatório em seus principais componentes, observa-se que a doença cerebrovascular foi a principal causa de morte, seguida pela doença isquêmica do coração. $^{2}$

Estudos que analisaram a variação geográfica da doença isquêmica do coração no mundo encontraram a participação de fatores ambientais entre os seus determinantes, destacando-se o processo de urbanização, as condições socioeconômicas da população e as mudanças no estilo de vida. ${ }^{3-7}$ Estudos brasileiros que avaliaram o risco de morte em decorrência de doenças 
Introdução

cardiovasculares concluíram que o risco está inversamente relacionado às condições socioeconômicas da população. 8,9

\subsection{Tabagismo e doença cardiovascular}

É importante ressaltar a influência do tabagismo na morbimortalidade geral e, em especial, no surgimento das doenças cardiovasculares. O tabagismo é a principal causa de morte evitável no mundo e o tabagismo passivo é a terceira causa. Estima-se que o tabagismo esteja associado a cerca de cinco milhões de mortes por ano no mundo. ${ }^{10}$ Proporcionalmente, é responsável por 1 em cada 5 mortes e, para os tabagistas que continuam a fumar até o final da vida, a chance de sofrer uma morte prematura devido a complicações do tabagismo é de aproximadamente $50 \% .{ }^{11}$ Além disso, o tabagista tem uma chance quinze vezes maior de desenvolver câncer de pulmão, onze vezes maior de desenvolver doenças pulmonares crônicas e duas vezes maior de sofrer um infarto agudo do miocárdio quando comparado a um indivíduo que não fuma. ${ }^{12}$

Considerando-se os tabagistas passivos, números expressivos em relação à doença coronariana também são observados. Estudos iniciais mostraram um aumento no risco relativo de infarto agudo do miocárdio de $25-31 \%$ em relação à população em geral $^{13-18}$ e um aumento no risco de acidente vascular cerebral em torno de $82 \%$. $^{19}$ Estudos recentes, que usaram marcadores biológicos para avaliar 
Introdução

a exposição à fumaça do cigarro mostram que o aumento do risco de infarto pode chegar a $60 \%$ entre os tabagistas passivos. ${ }^{20}$

\subsection{Tabagismo e disfunção endotelial}

Mecanismos pelos quais o tabagismo ativo e o tabagismo passivo poderiam induzir eventos cardiovasculares incluem: diminuição da oferta de oxigênio ao miocárdio, aumento da ativação plaquetária, alteração da função endotelial, aceleração do processo de aterosclerose e aumento da liberação de radicais livres, ${ }^{21}$ oxidação das lipoproteínas de baixa densidade, aumento da resistência à insulina, ${ }^{17}$ entre outros. Deve-se enfatizar que o risco de eventos cardiovasculares parece não apresentar relação linear com o nível de exposição ao tabaco 22,23 ou à fumaça do tabaco. Mesmo uma curta exposição está associada a um imediato aumento na agregabilidade plaquetária e a alterações na função endotelial - cerca de 30 minutos após a exposição estas alterações já podem ser notadas. ${ }^{17,24-27}$ Por outro lado, após a cessação do tabagismo observase uma redução no risco de infarto do miocárdio que não é proporcional ao tempo que o indivíduo parou de fumar - nota-se uma considerável redução no risco de infarto nas primeiras semanas a meses após a interrupção, ${ }^{28}$ que chega a 50\% após 1 ano sem fumar. 


\subsection{Estratégias para redução dos riscos associados ao tabagismo}

Pautando-se no fato de que o risco de infarto cai expressivamente no primeiro ano após a interrupção à exposição à fumaça do cigarro, e na experiência de pequenas localidades pelo mundo que proibiram o fumo parcial ou totalmente e observaram uma redução nas taxas de infarto do miocárdio, foi estruturada uma estratégia mundial para controle do consumo de tabaco.

\subsubsection{Convenção-Quadro para o Controle do Tabagismo}

A Convenção-Quadro para o Controle do Tabaco (CQCT) foi o primeiro tratado internacional de Saúde Pública da Organização Mundial da Saúde (OMS). Em maio de 1996 ela foi idealizada durante a Assembléia Mundial de Saúde. Em 2003, durante a 56ª Assembléia Mundial da Saúde, foi criada a CQCT. Em 27 de fevereiro de 2005 a Convenção-Quadro entrou em vigor para os países signatários. Trata-se de um instrumento legal pelo qual os países signatários se comprometeram a implantar ações integradas para controle do tabagismo em seus países e, conseqüentemente, no mundo. Entre estas ações, destacam-se a regulamentação ou banimento da publicidade, do patrocínio e da promoção de produtos de tabaco, a proteção contra a exposição à fumaça ambiental de tabaco (controle do tabagismo passivo), a promoção da cessação do tabagismo (controle do tabagismo ativo), a criação de alternativas para a fumicultura, a elevação dos 
Introdução

impostos sobre produtos fumígenos, o controle do mercado ilegal de tabaco, a realização de advertências sanitárias nos produtos que contenham derivados de tabaco, entre outros. ${ }^{29,30}$

A Convenção-Quadro estimulou o surgimento de leis anti fumo pelo mundo.

\subsubsection{Leis anti fumo}

Estudos prévios realizados em localidades do mundo que implantaram leis anti fumo verificaram reduções variáveis nas taxas de internação hospitalar por infarto agudo do miocárdio após o início da restrição ao fumo. Estas taxas variam entre 0 e $40 \%,{ }^{28,31-42}$ sendo que os melhores resultados foram observados nos estudos iniciais realizados em pequenas localidades norte americanas, enquanto que estudos maiores observaram reduções menos expressivas nessas taxas. Como exemplos, podemos citar a cidade de Nova lorque, que contabilizou uma redução em torno de $8 \%$ nas taxas de internação por infarto agudo do miocárdio, ${ }^{33}$ a Itália, com redução de cerca de $13 \%,{ }^{28}$ a Inglaterra, ${ }^{35}$ com redução de cerca de $2,4 \%$ e a Nova Zelândia, ${ }^{37}$ que não registrou redução na taxa de admissões hospitalares por infarto do miocárdio após a lei anti fumo. Enquanto isso, em localidades menores, como Helena, nos EUA, observou-se reduções mais expressivas, em torno de $40 \% .{ }^{34}$ Uma das explicações para este achado é que localidades menores têm maior facilidade no controle da população (somente um 
Introdução

hospital na cidade, em que há informações sobre os indivíduos que internam no hospital - se são cidadãos da cidade ou se provêm de outra localidade).

O principal objetivo das leis anti fumo é o benefício à saúde da população em geral, mas com especial melhoria da saúde das pessoas que não fumam, mas convivem com pessoas que fumam dentro de ambientes fechados (tabagistas passivos), principalmente nos locais de trabalho e em atividades de lazer, como idas a restaurantes, bares, lanchonetes e casas noturnas. Outro objetivo é a limitação dos locais em que os tabagistas ativos podem fumar e, com isso, tentar incentivá-los a deixar este vício ou pelo menos reduzir o consumo de produtos fumígenos.

\subsection{2.a. História das leis anti fumo no mundo}

A primeira proibição pública ao ato de fumar reconhecida na história data de 1590, quando o papa Urbano VII, em seu curto papado de 13 dias, ameaçou excomungar quem "tomasse tabaco no interior ou no adro de uma igreja, tanto por mastigá-lo, por fumá-lo com cachimbo ou por cheirá-lo em pó pelo nariz". A primeira ordenação municipal contra o cigarro e outros derivados de tabaco surgiu no Ducado da Alta Saxônia, na região da Bavária e em determinadas partes da Áustria, no século XVII. Entre as cidades de maior porte, a primeira lei anti fumo surgiu em Berlim, em 1723. Porém, cerca de um século depois, esta e muitas outras leis foram derrubadas nas revoluções liberais de 1848. Dentre as nações, a 
Introdução

primeira a impor uma restrição real ao fumo foi a Alemanha, país no qual o partido nazista liderou um movimento antitabaco intenso, com a proibição aos cidadãos de fumarem nas universidades, correios, hospitais militares, escritórios do partido nazista, bondes, ônibus e trens. Além disso, promoveu palestras aos soldados, aumentou os impostos sobre os cigarros e restringiu a propaganda. Após o término da segunda guerra mundial, houve um esmorecimento desta campanha antitabaco e o assunto voltou a crescer e se disseminar no mundo somente no final do século XX. Leis anti fumo, com a proibição de fumar em locais públicos (parcialmente ou integralmente), voltaram a surgir à partir de 1975, com o 'Minnesota Clean Indoor Air Act', que proibiu o fumo na maior parte dos locais de circulação pública, com exceção dos bares e restaurantes, na cidade americana de Minnesota. As leis se disseminaram a partir de 1990, quando estudos científicos começaram a mostrar os benefícios deste tipo de proibição, e, pela primeira vez, uma cidade norte americana passou a banir o cigarro de todos os locais de circulação pública, incluindo bares e restaurantes (cidade de San Luis Obispo, Califórnia). A Irlanda foi o primeiro país a ordenar uma lei proibindo o fumo em todos os locais de trabalho, em $2004 .^{43,44}$

Nas Américas, segundo a Organização Pan Americana de Saúde (OPAS), 16 países já estabeleceram ambientes livres de fumo em todos os locais públicos fechados e de trabalho: Argentina, Barbados, Canadá, Chile, Colômbia, Costa Rica, Equador, Guatemala, Honduras, Jamaica, Panamá, Peru, Suriname, Trinidad e Tobago, Uruguai e Venezuela. Recentemente, o Brasil se uniu a este 
Introdução

grupo. Outros países possuem restrições parciais ao fumo em locais fechados ou restrições em partes do território (cidades, estados ou regiões). O Uruguai foi o primeiro país latino americano a adotar uma lei nacional de restrição ao fumo em locais fechados, em $2006 .{ }^{45}$

\subsection{2.b. História das leis anti fumo no Brasil}

No Brasil, a luta contra o tabagismo tornou-se mais agressiva à partir de 1985, com a formação do Grupo Assessor ao Ministério da Saúde para o Controle do Tabagismo, que foi responsável pela elaboração do Programa Nacional de Combate ao Fumo, em 1986. ${ }^{46}$ Juntamente com o Canadá, o Brasil foi pioneiro na adoção de imagens ilustrativas dos malefícios do tabaco nos invólucros dos cigarros. A partir de 1980, por exemplo, algumas empresas, integrando-se, implantaram programas de combate ao tabaco entre seus funcionários. Na década de 90 , encontros científicos aconteceram por todo o Brasil, enfocando o combate ao fumo.

Um breve histórico da luta nacional contra o fumo está resumido abaixo:

- 1863: A Gazeta Médica do Rio de Janeiro publicou o trabalho "O abuso do tabaco como causa de angina do peito". Esta publicação é a primeira manifestação a respeito do assunto no âmbito científico e acadêmico brasileiro. ${ }^{47}$ 
- 1906: O município de São Paulo foi o pioneiro em legislar, promulgando a Lei no 947, datada de 25.10.1906, que proibia "a abertura das casas de fumo aos domingos e feriados". ${ }^{48}$

- 1965: Apresentado o primeiro Projeto de Lei instituindo a obrigatoriedade de advertência sobre os malefícios do fumo nas embalagens dos maços de cigarro.

- 1971: Surgiram projetos de lei com a finalidade de proibir parcialmente a propaganda do fumo em TV e Rádio. Sua regulamentação comercial reiterava a obrigatoriedade da advertência nos maços de cigarros e a não permissão da venda para menores. Neste mesmo ano, o Conselho Federal de Medicina, pela Resolução n. ${ }^{\circ}$ 440, de 26.03.71, proibiu o fumo em suas reuniões.

- 1979: acontece o "Seminário sobre tabagismo", em Salvador (BA). Desse evento, resultou a chamada Carta de Salvador, documento histórico em que médicos alertaram os poderes públicos, as instituições médicas e a população a respeito dos enormes malefícios produzidos no organismo pelo uso do tabaco. ${ }^{49}$ Neste mesmo ano foi estruturado o primeiro "Programa Nacional Contra o Fumo", coordenado pela Comissão Especial de Combate ao Tabagismo da Associação Médica Brasileira. ${ }^{50}$

- 1980: é desenvolvido material didático sobre Fumo e Saúde pela Rádio e Televisão Cultura de São Paulo para distribuição às escolas de $1^{\circ}$ e $2^{0}$ graus estaduais e municipais de São Paulo. ${ }^{51}$ 
Introdução

- 1981: Com o patrocínio da Addiction Research Foundation de Toronto Canadá, foi realizada a primeira determinação dos índices de nicotina, alcatrão e monóxido de carbono em cigarros utilizando-se metodologia científica. Duas marcas de cigarros nacionais foram testadas e os índices foram superiores aos divulgados pela indústria do tabaco.

- 1985: o Ministério da Saúde assumiu, oficialmente, a luta contra o tabagismo, criando o Grupo Assessor do Ministério da Saúde para Controle do Tabagismo no Brasil. Esta Comissão propôs o projeto de Lei ํo 7 488, para a instituição do Dia Nacional de Combate ao Fumo.

- 1986: o Congresso Nacional aprovou a Lei $n . \frac{0}{7}$ 488, de 11.06.1986, que instituiu o Dia Nacional de Combate ao Fumo.

- 1993: No dia 31 de maio ocorreu a Comemoração do Dia Mundial sem Tabaco pela primeira vez no INCOR e no Brasil, em parceria com o programa da OMS "Tobacco or Healthy".

- 1994: aconteceu o 1ํ Congresso Brasileiro sobre Tabagismo.

- 1996: aprovada a Lei oo 9 294, de 15.07.1996, que dispôs sobre as restrições ao uso e propaganda de produtos fumígenos, bebidas alcóolicas, medicamentos, terapias e defensores agrícolas. Aconteceu o $2^{\circ}$ Congresso Brasileiro sobre Tabagismo.

- 1998: Junto ao Departamento de Aviação Civil, houve a promoção e efetivação da proibição total de fumar nos aviões, em todas as viagens, com qualquer duração, no território nacional. 
Introdução

- 1999: aprovada a Lei no 9 782, de 26.01.1999, que dispôs sobre a Agência Nacional de Vigilância Sanitária (ANVISA), incluindo a regulamentação, o controle e a fiscalização dos produtos fumígenos derivados do tabaco.

- 2000: aprovada a Lei no 10167 de 27.12.2000, dando maior abrangência às medidas anti tabágicas, como restrição da propaganda de tabaco em televisão, rádio, jornais, revistas, outdoor e merchandising, limitando-a aos pontos internos de venda. Com relação ao patrocínio ao esporte, estabeleceu o prazo de dois anos para encerrar os contratos dos patrocínios esportivos. Aconteceu o $3^{\circ}$ Congresso Brasileiro sobre Tabagismo.

- 2003: o Conselho Federal de Medicina criou a Comissão de Controle do Tabagismo.

- 2005: faleceu o professor José Rosemberg, considerado o pioneiro do combate ao tabaco no Brasil.

- 2009: aprovada a lei $n^{0} 13541$ em São Paulo, que proibiu o fumo em locais fechados no estado de São Paulo.

O último e mais importante passo no combate ao fumo no Brasil ocorreu em 03 de dezembro de 2014, quando entrou em vigor a lei que proíbe o fumo em locais fechados em todo o território nacional. A lei ainda extinguiu os fumódromos e acabou com a possibilidade de propaganda comercial de cigarros, mesmo nos pontos de venda, onde até então era permitida publicidade nos locais de 
Introdução

armazenamento de cigarros. Permaneceu a permissão para a exposição dos produtos acompanhada por mensagens sobre os males provocados pelo fumo. Além disso, os fabricantes de cigarro foram obrigados a aumentar os espaços contendo avisos sobre os danos causados pelo tabaco nas embalagens dos maços de cigarro. Pela nova regra, a mensagem deveria ocupar $100 \%$ da face posterior das embalagens e de uma de suas laterais. Com o início da vigência da lei, permaneceu a permissão de fumar em residências particulares, em áreas ao ar livre, parques, praças, áreas abertas de estádios de futebol, vias públicas e tabacarias, que devem ser voltadas especificamente para esse fim. Entre as exceções estão também cultos religiosos, onde os fiéis podem fumar caso o fumo faça parte do ritual.

\subsection{2.c. A Lei anti fumo no estado de São Paulo}

No estado de São Paulo, a Lei no 13.541 , que foi aprovada em maio de 2009, proibiu o uso de cigarros e outros derivados de tabaco em locais fechados e/ou semi fechados, públicos e privados, excetuando-se residências, locais de culto religioso em que o fumo faça parte da cerimônia e locais destinados ao consumo de produtos fumígenos. O estado de São Paulo foi pioneiro no Brasil na adoção da lei anti fumo. Através desta medida, projetou-se uma redução na morbidade por doenças cardiovasculares similar à observada em outras localidades, nos meses que se sucederam à lei. 
Introdução

Para avaliar o impacto inicial desta lei, foi realizado um estudo prospectivo pelo Programa de Tratamento de Tabagismo do Instituto do Coração, Área de Cardiologia, em parceria com a Secretaria de Saúde do estado de São Paulo. Foram visitadas casas noturnas, bares, restaurantes, padarias e afins no município de São Paulo, com o intuito de avaliar o nível de CO ambiental e individual existente nestas localidades, gerado predominantemente pelo cigarro fumado dentro destes estabelecimentos. Esta medição foi realizada antes do inicio da vigência da lei anti fumo e, posteriormente, os mesmos estabelecimentos foram visitados cerca de 3 meses após o início da vigência da lei e a medição foi repetida. A avaliação consistiu na medição do $\mathrm{CO}$ ambiental, medido nestas localidades em três níveis - parte aberta, parte semi-aberta e parte fechada do estabelecimento - e na medição do $\mathrm{CO}$ exalado pelos trabalhadores destas localidades. ${ }^{52} \mathrm{~A}$ escolha da medição de CO foi realizada com base em dados preexistentes da relação entre exposição à fumaça do cigarro e elevação das taxas de $\mathrm{CO}$ - tanto no ar exalado quanto no ambiente em que há pessoas fumando. ${ }^{53-55}$

Este estudo demonstrou, de maneira inédita, uma significativa redução nas taxas de $\mathrm{CO}$ em todos os níveis avaliados (parte aberta, semi aberta e fechada do estabelecimento), tanto em restaurantes, quanto em casas noturnas, bares e demais estabelecimentos, bem como nos trabalhadores destas localidades, fossem eles não tabagistas ou tabagistas, independentemente de terem parado de fumar ou não durante este período. ${ }^{52}$ 
Introdução

Concomitantemente, iniciou-se esta segunda fase da pesquisa, com 0 levantamento dos dados de mortalidade e internações hospitalares em decorrência de doenças cardiovasculares e cerebrovasculares comparando o período anterior e posterior à lei anti fumo.

1.4. Interferentes externos que podem influenciar na taxa de eventos cardiovasculares

Fatores adicionais, como temperatura, umidade do ar, poluição ambiental, introdução de novos medicamentos para prevenção de eventos cardiovasculares ou novas tecnologias para diagnóstico de doença cardiovascular, sazonalidade dos eventos cardiovasculares e cerebrovasculares também tem demonstrado influenciar a ocorrência de infarto do miocárdio e outras síndromes coronarianas agudas e acidentes vasculares cerebrais em diversos estudos. Nos meses de inverno e primavera há uma tendência a aumentar a ocorrência de eventos, ${ }^{56-59}$ enquanto no verão observa-se a tendência oposta. Em virtude deste fato, alguns estudos prévios que avaliaram o impacto de leis anti fumo na ocorrência de eventos cardiovasculares e cerebrovasculares levaram em consideração estes outros fatores não relacionados diretamente com as leis anti-fumo, mas que poderiam surgir como variáveis de confusão.

Alguns autores, considerando as oscilações sazonais dos infartos, realizaram o ajuste fazendo uma comparação entre os mesmos meses em anos 
Introdução

subseqüentes (antes e depois do inicio da vigência da lei). ${ }^{34,36,38,39,60}$ Outros estudos, como o estudo suíço que avaliou o impacto da lei anti fumo na cidade de Graubünden, avaliaram se o nível de poluição ambiental (mensurada através da medida de material particulado inalável menor que $\left.10 \mu \mathrm{m}-\mathrm{MP}_{10}\right)^{61-63}$ e a introdução de hipolipemiantes orais tiveram influência na ocorrência dos eventos cardiovasculares concomitantemente à lei anti fumo. Neste estudo, não foi verificada interferência da poluição ou do uso de hipolipemiantes orais na magnitude da ocorrência de eventos cardiovasculares. ${ }^{61}$

No geral, poucos estudos prévios que avaliaram os efeitos das leis anti fumo levaram em consideração dados de poluição ambiental, bem como poucos estudos realizaram a avaliação conjunta da interferência da lei anti fumo nas internações por acidente vascular cerebral. Adicionalmente, poucos estudos comprovaram uma redução nas taxas de mortalidade por infarto e acidente vascular cerebral. Somente um país na América do Sul avaliou o efeito da lei anti fumo nas taxas de internação hospitalar por infarto agudo do miocárdio ${ }^{45} \mathrm{e}$ nenhum avaliou a influência nas taxas de internação por acidente vascular cerebral em conjunto.

Neste estudo, avaliamos a incidência de eventos cardiovasculares e cerebrovasculares (taxas de internação e mortalidade por infarto agudo do miocárdio e outras síndromes coronarianas agudas e acidente vascular cerebral) antes e após a implantação da lei anti fumo no município de São Paulo, avaliando 
Introdução

conjuntamente os dados ambientais de poluição atmosférica, umidade do ar e temperatura e considerando a sazonalidade dos eventos.

O fato de termos observado uma redução significativa na concentração de monóxido de carbono em locais de grande circulação de pessoas, como bares e restaurantes, foi um marcador da diminuição real do tabagismo passivo nestes locais, e, com isso, esperamos encontrar uma redução nas taxas de internação e mortalidade por eventos cardiovasculares e cerebrovasculares após o início da vigência da lei. 


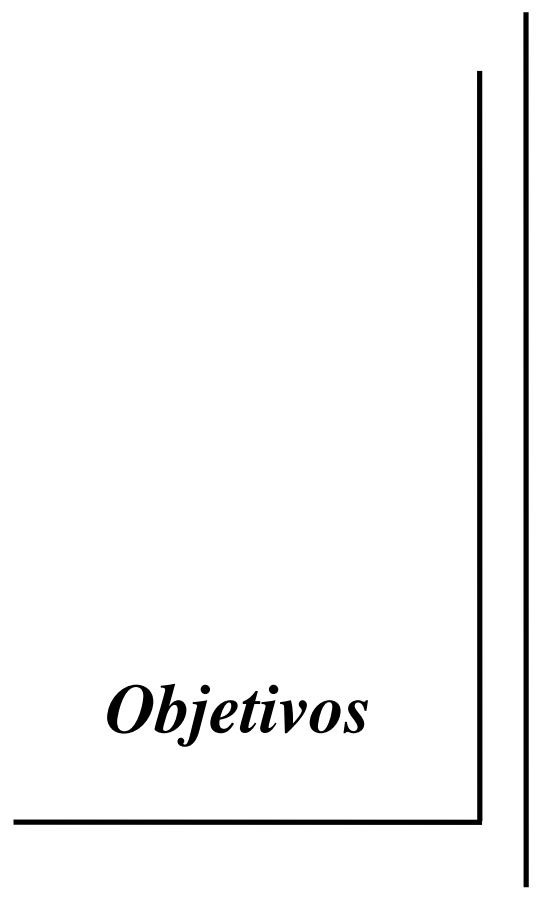


Objetivos

\section{OBJETIVOS}

Avaliar o impacto da lei antifumo nas taxas de internação hospitalar e de mortalidade devido a infarto agudo do miocárdio e síndromes coronarianas agudas correlatas (CIDs I21, I22, I23, I24) e devido a acidente vascular cerebral (CIDs I63, 164) no município de São Paulo. 


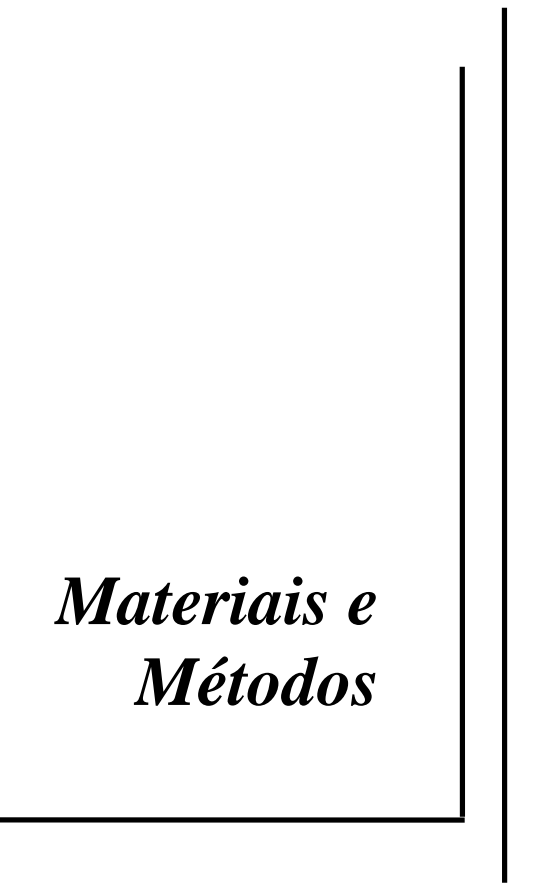




\section{MATERIAIS E MÉTODOS}

\subsection{Tipo de estudo}

Trata-se de um estudo ecológico de séries temporais aprovado em 2009 pela Comissão de Ética para Análise de Projetos de Pesquisa (CAPPesq) do Hospital das Clínicas da Faculdade de Medicina da Universidade de São Paulo (HC-FMUSP), que utilizou como base a população residente do município de São Paulo.

Foram avaliadas as taxas de mortalidade e internação em decorrência de doença cardiovascular e cerebrovascular, segundo patologias especificadas na Classificação Estatística Internacional de Doenças e Problemas Relacionados a Saúde, 10ª revisão, com 3 dígitos (CID 103 dígitos), no município de São Paulo. O levantamento foi realizado no período de janeiro de 2005 a dezembro de 2010, sendo que a lei anti fumo foi implantada em agosto de 2009.

\subsection{Coleta de dados}

Os dados de mortalidade foram obtidos a partir do Sistema de Informação sobre Mortalidade (SIM) do Programa de Aprimoramento das Informações de Mortalidade (Pro-AIM), da Secretaria Municipal de Saúde de São Paulo. Foram estudados conjuntamente os CIDs I21 (infarto agudo do miocárdio), I22 (infarto do 
Materiais e Métodos

miocárdio recorrente), I23 (complicações atuais subsequentes ao infarto agudo do miocárdio) e I24 (outras doenças isquêmicas agudas do coração), nomeados conjuntamente "Infarto" e os CIDs I63 (acidente vascular cerebral isquêmico) e 164 (acidente vascular cerebral, não classificado como isquêmico ou hemorrágico), nomeados conjuntamente "AVC".

Os dados de morbidade foram obtidos através do Sistema de Informações Hospitalares (SIH) do Ministério da Saúde, com base no sistema DATASUS, através do levantamento dos mesmos CIDs obtidos para mortalidade. O número total de internações hospitalares ocorridas no Sistema Único de Saúde (SUS) nos períodos estudados também foi obtido e recebeu a designação de "leitos".

A população residente no município de São Paulo foi obtida na Fundação SEADE (Sistema Estadual de Análise de Dados), responsável pelas estimativas da população a partir dos censos demográficos decenais do IBGE (Instituto Brasileiro de Geografia e Estatística).${ }^{64}$ Os dados compreendidos entre os censos foram estimados utilizando-se modelo de crescimento geométrico da população.

Fórmula para o cálculo de progressão geométrica da população:

$$
P n=P 0^{*}(1+r)^{n}
$$

Onde:

$P 0=$ população do município de São Paulo em julho de 2000

$P n=$ população do município de São Paulo após $n$ meses em relação ao mês 0

$r=$ taxa de crescimento geométrico

$n=$ número de meses decorridos após o mês 0 
A população atendida pelo Sistema Único de Saúde (SUS) no município de São Paulo representa uma parcela da população total do município de São Paulo. Em 2010, foi realizado levantamento pela Secretaria Municipal de Saúde, que estimou em $55,6 \%$ a população atendida exclusivamente pelo SUS ${ }^{65}$ (os outros $44,4 \%$ da população apresentam algum tipo de cobertura pelo sistema suplementar de saúde). Em nossa análise, utilizamos esta porcentagem da população que apresenta cobertura exclusiva do SUS para o cálculo das taxas mensais de internação por "Infarto" e "AVC". Para o cálculo das taxas mensais de mortalidade, o denominador utilizado foi a população total do município de São Paulo. As taxas foram obtidas para cada 100000 habitantes.

Informações sobre nível de monóxido de carbono (CO), medido em partes por milhão (ppm) e de outros poluentes como material particulado inalável (MP10), dióxido de enxofre (SO2), ozônio (O3) e dióxido de nitrogênio (NO2), medidos em micrograma por metro cúbico $\left(\mu \mathrm{g} / \mathrm{m}^{3}\right)$, além de temperatura $(T)$, medida em graus Celsius $\left({ }^{\circ} \mathrm{C}\right)$ e umidade relativa do ar (UR), medida em percentual (\%) foram obtidos junto à Companhia Ambiental do Estado de São Paulo (CETESB), os quais foram usados para avaliar a influência da poluição ambiental, temperatura e umidade relativa do ar nos desfechos em estudo. Essas variáveis são extraídas perdiodicamente das estações de medição fixas do município de São Paulo pela CETESB. Os dados foram agregados e analisados mensalmente, uma vez que há uma típica variação sazonal dos poluentes, bem como da umidade do ar e da temperatura. O nível de $\mathrm{CO}$ considerado foi a média mensal do $\mathrm{CO}$ máximo atingido a cada 8 horas. Para temperatura, foram medidas a temperatura média 
máxima e a temperatura média mínima e para umidade relativa do ar foi considerada a média mínima. Foram coletados dados mensais entre janeiro de 2005 e dezembro de 2010.

\subsection{Análise dos dados}

Foi utilizada a planilha eletrônica MS-Excel®, em sua versão do MS-Office® 2010, para a organização inicial dos dados.

A metodologia Box-Jenkins ${ }^{66}$ foi aplicada às séries de taxas de internações hospitalares e de taxas de mortalidade, possibilitando a análise conjunta dessas taxas com os dados ambientais e com o total de leitos hospitalares nos períodos estudados.

\subsubsection{Modelos e programas estatísticos}

Os modelos de função de transferência ARIMAX (modelos Autorregressivos, Integrados e de Médias Móveis, com variáveis exógenas) foram estimados usando o comando arima do "Data Analysis and Statistical Software" Stata/LP, StataCorp $®$, versão 10.1. Os procedimentos de estimação seguiram as recomendações de McDowell. ${ }^{67}$

Os modelos de intervenção em séries temporais foram conduzidos usando o comando itsa (interrupted time series analysis) do Stata/MP, versão 14, e seguiram os procedimentos descritos por Linden. ${ }^{68}$ 


\subsubsection{Modelo ARIMAX}

O processo de modelagem seguiu conforme descrito em Masi ${ }^{69}$ em que a metodologia de Box-Jenkins foi aplicada. Primeiro identificou-se o modelo mais adequado segundo as estruturas de autocorrelações e a presença de raízes unitárias. Todos esses procedimentos foram realizados no software Stata/LP, versão 10.1, e os comandos usados são descritos entre parênteses. As ordens autorregressivas $p$, de médias móveis $q$, assim como suas correspondentes ordens sazonais $P$ e $Q$ foram identificadas pela observação das funções de autocorrelação (ac) e autocorrelação parcial (pac). Para auxiliar na identificação da maior ordem autorregressiva foi utilizado o teste modificado de Dickey-Fuller (dfgls) e o comando varsoc (vector autoregressive order selection criterion), os quais usam o critério de informação de Akaike (AIC) para determiná-la. A estacionaridade das séries foi testada com o teste aumentado de Dickey-Fuller (dfuller) e com o teste de Phillips-Perron (pperron) para raízes unitárias. A correlação temporal entre as séries de entrada e as séries de saída foi estimada usando a função de correlação cruzada (xcorr). Para auxiliar na identificação da ordem das correlações realizou-se o pré-branqueamento ${ }^{1}$ das séries e a estimação da função de correlação cruzada.

Depois de identificadas as estruturas de autocorrelação e de correlações cruzadas, partiu-se para a construção dos modelos de função de transferência.

1 Pré-branqueamento é um método estatístico desenvolvido por Box e Jenkins para testar as correlações cruzadas entre duas séries. O procedimento consiste em ajustar um modelo ARIMA à variável de entrada e em seguida aplicar o mesmo modelo à variável de saída. 
Primeiro entraram as séries indicadoras do clima (temperatura e umidade relativa do ar). Em seguida, entraram as séries indicadoras dos poluentes atmosféricos (CO, MP10, NO2, SO2, O3). Após ajustado o modelo, entrou a série de intervenção (lei anti fumo), que recebeu o valor 0 para o período anterior a agosto de 2009 e 1 para o período posterior a essa data, caracterizando-a como intervenção do tipo "degrau" (step function). Com isso, o efeito esperado da intervenção foi a mudança permanente ou temporária no nível e/ou inclinação da série. Durante todo o processo o modelo foi sendo ajustado para encontrar as séries e suas respectivas defasagens que melhor explicassem o agravo de interesse. Após cada ajuste o modelo foi reestimado e as medidas de ajuste foram checadas novamente. Devido à multicolinearidade entre as séries de poluentes, optou-se por manter no modelo aquela que fornecia a melhor previsão do fenômeno em estudo. Séries de entrada sem efeito estatisticamente significativo ou sem plausibilidade biológica foram descartadas. Nesse contexto, "sem efeito significativo" menciona as séries cujo comportamento não contribuiu para a previsão da série de saída ou efeito de confundimento, ou seja, uma dada série de entrada é correlacionada à série de saída e a outra série de entrada. ${ }^{69}$

Primeiro, foi estimado um modelo geral que contemplasse todo o período de estudo (janeiro de 2005 a dezembro de 2010) para avaliar o efeito da intervenção. Em seguida, foi estimado outro modelo com os dados do período anterior à intervenção (janeiro de 2005 a julho de 2009) e, a partir desse modelo, fez-se a previsão fora da série para o período posterior à intervenção (agosto de 2009 a dezembro de 2010). A partir dessa previsão comparou-se o 
Materiais e Métodos

comportamento esperado da série sem a intervenção com o observado, que leva em consideração a ocorrência da intervenção.

\subsubsection{Modelo ITSA}

Com a finalidade de entender melhor e modelar o efeito da intervenção, outras duas técnicas de modelagem foram adotadas, tendo como referência o artigo original de Linden. ${ }^{68}$ Nesses métodos, os modelos são estimados usando regressão com mínimos quadrados ordinários, os quais, segundo os autores, são mais flexíveis e amplamente aplicados à análise de intervenção em séries temporais (ITSA).

O método Prais-Winsten and Cochrane-Orcutt regression (prais) foi utilizado para a estrutura de autocorrelações. Este método estima os parâmetros em um modelo linear no qual os resíduos são serialmente correlacionados. Especificamente, assume-se que os erros seguem um processo autorregressivo de primeira ordem. ${ }^{70}$ Para cada série de saída dois modelos foram estimados, um univariado e outro multivariado, o qual seguiu a estrutura de correlações cruzadas identificadas para os modelos de função de transferência descritos acima. Nesses dois casos, o intuito era verificar se houve alteração do nível e/ou tendência da série após a adoção da intervenção.

Por último, o procedimento itsa foi incorporado ao método ARIMAX, com a finalidade de estimar a presença de tendência nas séries de saída antes e após a 
intervenção. Neste caso também foram mantidas as estruturas de autocorrelação e correlações cruzadas identificadas nos modelos de função de transferência, conforme descrito anteriormente.

\subsubsection{Escolha do melhor modelo}

A checagem da acurácia dos modelos foi verificada pela análise do correlograma dos resíduos (corrgram), que fornece as funções de autocorrelação (acf) e autocorrelação parcial (pacf) e pela estatística $Q$, do teste $Q$ de Ljung-Box, que checa se os resíduos são ruído branco, e pelo teste de Shapiro-Wilk (swilk), para testar a adesão à distribuição normal dos resíduos. O melhor modelo foi escolhido baseando-se no critério de informação de Akaike (AIC), no erro quadrático médio de previsão (EQMP) e no desvio padrão residual, conforme estabelecido por Christopher (2000) e Morettin e Toloi (2006). Além disso, seguindo a filosofia de Box-Jenkins, sempre se optou pelos modelos mais parcimoniosos, ou seja, aqueles com o menor número de parâmetros. ${ }^{69} \mathrm{Em}$ todas as análises adotou-se o nível de significância de $5 \%(p<0,05)$.

\subsubsection{Diferenciação entre ARIMAX e ITSA-ARIMAX}

A metodologia ARIMAX permite uma avaliação integrada completa dos dados, permitindo a comparação, momento a momento, entre a ocorrência real 
Materiais e Métodos

dos eventos e a previsão de ocorrência dos mesmos, segundo o modelo estimado. Por outro lado, a metodologia ITSA-ARIMAX permite uma avaliação pontual do momento da intervenção, tornando possível avaliar se a lei anti fumo produz um efeito imediato, além de avaliar a presença de efeito posterior à mesma. Devido à menor complexidade, a metodologia ITSA é a mais utilizada em trabalhos científicos com leis anti fumo prévias a esta. 


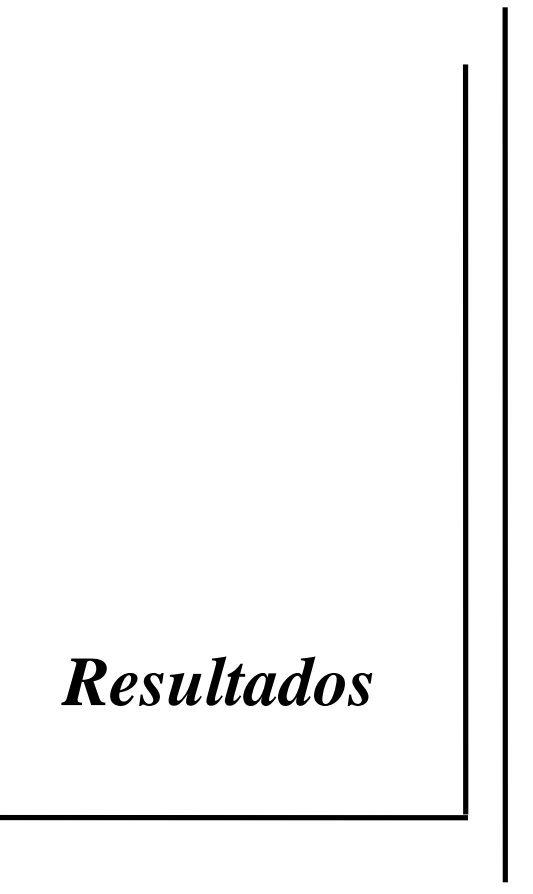


Resultados

\section{RESULTADOS}

\subsection{Análise da morbidade hospitalar}

O número de internações hospitalares do Sistema Único de Saúde (SUS) foi obtido para "Infarto" (Tabela 1), "AVC" (Tabela 2) e "leitos" (Tabela 3).

Tabela 1 - Número mensal de internações hospitalares ocorridas em hospitais do SUS com diagnóstico de "Infarto" (CIDs 121, I22, I23 e I24), Município de São Paulo - jan. 2005 a dez. 2010

\begin{tabular}{lcccccc}
\hline \multicolumn{1}{c}{ MÊS } & 2005 & 2006 & 2007 & 2008 & 2009 & 2010 \\
\hline Janeiro & 838 & 866 & 888 & 714 & 829 & 921 \\
Fevereiro & 734 & 758 & 847 & 629 & 731 & 852 \\
Março & 835 & 823 & 879 & 763 & 867 & 964 \\
Abril & 710 & 875 & 841 & 810 & 904 & 916 \\
Maio & 800 & 907 & 1005 & 888 & 927 & 1080 \\
Junho & 880 & 897 & 908 & 900 & 978 & 987 \\
Julho & 982 & 929 & 974 & 950 & 921 & 1013 \\
Agosto & 980 & 965 & 965 & 870 & 927 & 1052 \\
Setembro & 801 & 919 & 793 & 870 & 871 & 942 \\
Outubro & 757 & 910 & 853 & 908 & 896 & 970 \\
Novembro & 868 & 840 & 724 & 758 & 868 & 943 \\
Dezembro & 747 & 877 & 640 & 704 & 828 & 886 \\
\hline FONTE: Sech
\end{tabular}

FONTE: Secretaria Estadual de Saúde 
Resultados

Tabela 2 - Número mensal de internações hospitalares ocorridas em hospitais do SUS com diagnóstico de "AVC" (CIDs 163 e I64), Município de São Paulo - jan. 2005 a dez. 2010

\begin{tabular}{lcccccc}
\hline \multicolumn{1}{c}{ MÊS } & 2005 & 2006 & 2007 & 2008 & 2009 & 2010 \\
\hline Janeiro & 496 & 557 & 538 & 534 & 497 & 570 \\
Fevereiro & 488 & 444 & 524 & 499 & 460 & 568 \\
Março & 616 & 548 & 635 & 440 & 538 & 641 \\
Abril & 576 & 530 & 536 & 483 & 550 & 593 \\
Maio & 597 & 568 & 705 & 525 & 559 & 595 \\
Junho & 598 & 594 & 587 & 565 & 655 & 641 \\
Julho & 636 & 554 & 628 & 544 & 639 & 578 \\
Agosto & 598 & 590 & 719 & 563 & 596 & 598 \\
Setembro & 550 & 574 & 574 & 552 & 582 & 639 \\
Outubro & 569 & 573 & 586 & 586 & 598 & 656 \\
Novembro & 580 & 615 & 558 & 482 & 560 & 556 \\
Dezembro & 566 & 595 & 453 & 571 & 529 & 587 \\
\hline
\end{tabular}

FONTE: Secretaria Estadual de Saúde

Tabela 3 - Número mensal total de internações hospitalares ocorridas em hospitais do SUS, Município de São Paulo - jan. 2005 a dez. 2010

\begin{tabular}{lcccccc}
\hline \multicolumn{1}{c}{ MÊS } & 2005 & 2006 & 2007 & 2008 & 2009 & 2010 \\
\hline Janeiro & 41255 & 44124 & 45393 & 41852 & 41148 & 46078 \\
Fevereiro & 39256 & 39281 & 42773 & 39471 & 39918 & 43938 \\
Março & 46235 & 48340 & 50430 & 44569 & 49408 & 53536 \\
Abril & 44300 & 45105 & 46980 & 45372 & 45777 & 48889 \\
Maio & 46689 & 49152 & 49376 & 46087 & 48001 & 50626 \\
Junho & 45373 & 45205 & 45991 & 45432 & 47306 & 48198 \\
Julho & 44138 & 46303 & 47006 & 47017 & 48438 & 48521 \\
Agosto & 46869 & 47808 & 49048 & 46564 & 49415 & 51284 \\
Setembro & 43837 & 44571 & 45548 & 45255 & 48085 & 48014 \\
Outubro & 44430 & 46012 & 47266 & 45364 & 47916 & 48451 \\
Novembro & 43261 & 43207 & 40909 & 40791 & 45178 & 47782 \\
Dezembro & 39788 & 42564 & 37222 & 39873 & 42658 & 45837 \\
\hline
\end{tabular}

FONTE: Secretaria Estadual de Saúde 
Resultados

Para se determinar as taxas mensais de internações hospitalares do SUS no período estudado, foi utilizada, no denominador, a população estimada usuária do SUS no município de São Paulo (tabela 4).

Tabela 4 - Estimativa da população SUS exclusiva do Município de São Paulo jan. 2005 a dez. 2010

\begin{tabular}{lcccccc}
\hline \multicolumn{1}{c}{ MÊS } & 2005 & 2006 & 2007 & 2008 & 2009 & 2010 \\
\hline Janeiro & 5993182 & 6040299 & 6087787 & 6135648 & 6183885 & 6232501 \\
Fevereiro & 5997094 & 6044242 & 6091761 & 6139653 & 6187922 & 6236570 \\
Março & 6001009 & 6048188 & 6095738 & 6143661 & 6191961 & 6240641 \\
Abril & 6004927 & 6052136 & 6099717 & 6147671 & 6196003 & 6244715 \\
Maio & 6008847 & 6056087 & 6103699 & 6151685 & 6200048 & 6248791 \\
Junho & 6012769 & 6060040 & 6107683 & 6155701 & 6204095 & 6252871 \\
Julho & 6016694 & 6063996 & 6111670 & 6159719 & 6208145 & 6256952 \\
Agosto & 6020622 & 6067955 & 6115660 & 6163740 & 6212198 & 6261037 \\
Setembro & 6024552 & 6071916 & 6119652 & 6167764 & 6216253 & 6265124 \\
Outubro & 6028485 & 6075880 & 6123647 & 6171790 & 6220311 & 6269214 \\
Novembro & 6032421 & 6079846 & 6127645 & 6175819 & 6224372 & 6273307 \\
Dezembro & 6036359 & 6083815 & 6131645 & 6179851 & 6228435 & 6277402 \\
\hline
\end{tabular}

FONTE: Fundação SEADE, Censos demográficos - IBGE, Secretaria Municipal de Saúde

\subsection{Análise de mortalidade}

Os dados de mortalidade também foram separados entre "Infarto" (tabela 5) e "AVC" (tabela 6). 
Resultados

Tabela 5 - Número mensal de óbitos por "Infarto" (CIDs I21, I22, I23 e I24), Município de São Paulo - jan. 2005 a dez. 2010

\begin{tabular}{lllllll}
\hline \multicolumn{1}{c}{ MÊS } & 2005 & 2006 & 2007 & 2008 & 2009 & 2010 \\
\hline Janeiro & 438 & 469 & 455 & 484 & 496 & 736 \\
Fevereiro & 401 & 431 & 423 & 421 & 434 & 671 \\
Março & 458 & 443 & 491 & 492 & 477 & 706 \\
Abril & 447 & 451 & 448 & 511 & 500 & 719 \\
Maio & 525 & 590 & 582 & 634 & 514 & 837 \\
Junho & 485 & 599 & 607 & 564 & 589 & 727 \\
Julho & 529 & 562 & 604 & 570 & 547 & 813 \\
Agosto & 520 & 557 & 608 & 544 & 551 & 758 \\
Setembro & 452 & 517 & 508 & 551 & 467 & 722 \\
Outubro & 448 & 476 & 538 & 541 & 498 & 730 \\
Novembro & 529 & 442 & 487 & 476 & 386 & 779 \\
Dezembro & 527 & 458 & 526 & 459 & 497 & 745 \\
\hline
\end{tabular}

FONTE: SIM - Pro-AIM

Tabela 6 - Número mensal de óbitos por "AVC" (CIDs 163 e 164), Município de São Paulo - jan. 2005 a dez. 2010

\begin{tabular}{lcccccc}
\hline \multicolumn{1}{c}{ MÊS } & 2005 & 2006 & 2007 & 2008 & 2009 & 2010 \\
\hline Janeiro & 149 & 218 & 228 & 229 & 219 & 244 \\
Fevereiro & 135 & 187 & 171 & 240 & 215 & 206 \\
Março & 124 & 209 & 222 & 224 & 240 & 232 \\
Abril & 136 & 196 & 206 & 236 & 273 & 215 \\
Maio & 156 & 245 & 235 & 241 & 240 & 236 \\
Junho & 121 & 254 & 272 & 257 & 256 & 277 \\
Julho & 142 & 260 & 234 & 285 & 295 & 243 \\
Agosto & 119 & 248 & 254 & 230 & 255 & 230 \\
Setembro & 135 & 218 & 246 & 229 & 233 & 232 \\
Outubro & 141 & 197 & 213 & 258 & 218 & 257 \\
Novembro & 125 & 226 & 197 & 210 & 231 & 236 \\
Dezembro & 109 & 221 & 245 & 247 & 221 & 252 \\
\hline FONTE: SIM & - Pro-AIM & & & &
\end{tabular}

FONTE: SIM - Pro-AIM

\subsection{Avaliação dos dados da CETESB}

Os dados coletados junto à CETESB foram organizados na tabela 7 . 
Resultados

Tabela 7 - Valores médios mensais da concentração de poluentes e variáveis ambientais, Município de São Paulo - jan. 2005 a dez. 2010

\begin{tabular}{|c|c|c|c|c|c|c|c|c|}
\hline MÊS/ANO & $\mathrm{CO}$ & MP10 & NO2 & 03 & $\mathrm{SO} 2$ & Tmax & Tmin & UR \\
\hline Jan/05 & 2,0 & 29,9 & 70,7 & 73,6 & 7,3 & 26,4 & 19,5 & 59,7 \\
\hline Fev/05 & 2,1 & 33,8 & 100,0 & 94,0 & 9,7 & 27,6 & 18,2 & 44,0 \\
\hline Mar/05 & 2,4 & 35,5 & 97,5 & 80,0 & 9,7 & 27,0 & 19,0 & 49,3 \\
\hline $\mathrm{Abr} / 05$ & 2,4 & 39,1 & 115,5 & 94,2 & 11,3 & 27,4 & 18,9 & 45,0 \\
\hline Mai/05 & 3,0 & 44,3 & 113,5 & 66,8 & 10,0 & 25,2 & 15,7 & 41,0 \\
\hline Jun/05 & 3,5 & 50,4 & 127,0 & 54,4 & 12,7 & 23,3 & 15,1 & 48,0 \\
\hline Jul/05 & 3,2 & 44,3 & 103,0 & 59,4 & 9,7 & 21,9 & 13,5 & 48,0 \\
\hline Ago/05 & 3,1 & 53,1 & 125,7 & 87,7 & 11,3 & 25,0 & 14,9 & 39,7 \\
\hline Set/05 & 2,0 & 28,4 & 95,0 & 68,2 & 7,3 & 22,8 & 14,3 & 58,3 \\
\hline Out/05 & 2,3 & 33,9 & 106,5 & 87,2 & 9,0 & 25,3 & 17,3 & 56,0 \\
\hline Nov/05 & 1,7 & 28,8 & 71,0 & 81,2 & 6,3 & 24,2 & 16,6 & 53,7 \\
\hline Dez/05 & 1,6 & 27,1 & 71,8 & 79,2 & 8,5 & 25,7 & 17,1 & 52,3 \\
\hline Jan/06 & 1,7 & 33,9 & 89,5 & 117,6 & 6,8 & 28,3 & 19,4 & 48,7 \\
\hline $\mathrm{Fev} / 06$ & 1,9 & 30,2 & 85,0 & 83,7 & 7,0 & 27,4 & 19,8 & 52,7 \\
\hline Mar/06 & 1,9 & 30,8 & 84,5 & 83,6 & 6,7 & 27,4 & 19,1 & 51,3 \\
\hline $\mathrm{Abr} / 06$ & 1,8 & 34,6 & 82,8 & 81,4 & 8,3 & 25,1 & 16,8 & 49,3 \\
\hline Mai/06 & 2,4 & 39,9 & 97,0 & 70,8 & 8,7 & 22,1 & 14,1 & 52,5 \\
\hline Jun/06 & 3,0 & 47,9 & 130,5 & 66,5 & 11,0 & 22,8 & 13,9 & 52,0 \\
\hline Jul/06 & 4,1 & 65,1 & 143,0 & 78,0 & 14,0 & 24,3 & 13,4 & 47,0 \\
\hline Ago/06 & 3,2 & 51,5 & 128,4 & 79,3 & 12,0 & 25,0 & 14,2 & 45,0 \\
\hline Set/06 & 2,3 & 40,3 & 116,8 & 90,8 & 10,5 & 24,7 & 15,2 & 49,5 \\
\hline Out/06 & 1,7 & 31,4 & 97,3 & 84,8 & 7,5 & 25,7 & 17,3 & 55,0 \\
\hline Nov/06 & 1,7 & 30,8 & 96,2 & 77,3 & 8,0 & 26,2 & 17,7 & 54,5 \\
\hline Dez/06 & 1,6 & 27,0 & 80,4 & 77,7 & 7,0 & 27,8 & 19,7 & 55,5 \\
\hline Jan/07 & 1,5 & 24,2 & 69,0 & 63,0 & 7,5 & 26,8 & 19,8 & 60,0 \\
\hline $\mathrm{Fev} / 07$ & 1,7 & 31,8 & 82,8 & 92,9 & 8,5 & 29,2 & 19,9 & 50,5 \\
\hline Mar/07 & 1,9 & 42,8 & 106,5 & 120,0 & 11,5 & 30,2 & 19,5 & 43,0 \\
\hline Abr/07 & 1,8 & 34,3 & 86,3 & 83,6 & 8,0 & 26,8 & 19,0 & 54,0 \\
\hline Mai/07 & 2,6 & 37,2 & 96,5 & 56,1 & 10,0 & 22,6 & 14,6 & 54,5 \\
\hline Jun/07 & 3,8 & 55,9 & 116,3 & 65,9 & 12,0 & 24,4 & 13,7 & 44,5 \\
\hline Jul/07 & 3,0 & 50,0 & 106,7 & 56,9 & 11,5 & 21,8 & 12,6 & 51,5 \\
\hline Ago/07 & 3,1 & 52,3 & 116,6 & 80,7 & 13,0 & 25,5 & 13,8 & 41,0 \\
\hline Set/07 & 2,0 & 53,4 & 99,0 & 110,4 & 9,5 & 27,3 & 16,1 & 34,0 \\
\hline Out/07 & 1,9 & 47,9 & 89,6 & 106,7 & 9,0 & 27,7 & 17,9 & 46,0 \\
\hline Nov/07 & 1,5 & 29,9 & 71,1 & 85,6 & 6,0 & 26,0 & 17,4 & 49,3 \\
\hline Dez/07 & 1,5 & 31,8 & 73,6 & 112,8 & 7,0 & 28,4 & 18,8 & 45,7 \\
\hline Jan/08 & 1,3 & 26,6 & 60,3 & 76,3 & 5,0 & 27,2 & 18,8 & 49,3 \\
\hline $\mathrm{Fev} / 08$ & 1,6 & 32,2 & 65,6 & 84,8 & 6,5 & 28,9 & 19,4 & 45,3 \\
\hline
\end{tabular}

continua 
Resultados

\begin{tabular}{|c|c|c|c|c|c|c|c|c|}
\hline MÊS/ANO & $\mathrm{CO}$ & MP10 & $\mathrm{NO} 2$ & $\mathrm{O} 3$ & $\mathrm{SO} 2$ & Tmax & Tmin & UR \\
\hline Mar/08 & 1,5 & 31,6 & 66,7 & 81,0 & 7,0 & 28,0 & 18,9 & 46,7 \\
\hline Abr/08 & 2,0 & 35,6 & 77,0 & 61,3 & 7,5 & 26,5 & 17,5 & 47,7 \\
\hline Mai/08 & 2,3 & 47,0 & 90,4 & 59,1 & 10,5 & 23,5 & 14,1 & 38,0 \\
\hline Jun/08 & 2,6 & 46,1 & 85,9 & 49,4 & 11,0 & 23,1 & 14,3 & 44,3 \\
\hline Jul/08 & 3,2 & 66,9 & 105,7 & 80,8 & 12,5 & 26,1 & 11,7 & 33,5 \\
\hline Ago/08 & 2,6 & 46,8 & 99,5 & 77,4 & 9,5 & 25,4 & 14,7 & 48,5 \\
\hline Set/08 & 1,7 & 35,6 & 85,8 & 75,3 & 10,5 & 24,3 & 14,6 & 49,5 \\
\hline Out/08 & 1,6 & 31,4 & 85,4 & 95,4 & 8,0 & 27,1 & 17,4 & 52,5 \\
\hline Nov/08 & 1,3 & 26,8 & 66,1 & 85,2 & 7,5 & 26,9 & 17,6 & 53,5 \\
\hline Dez/08 & 1,2 & 28,8 & 68,0 & 98,2 & 7,0 & 27,8 & 17,4 & 48,5 \\
\hline Jan/09 & 1,3 & 23,8 & 66,2 & 72,3 & 6,5 & 27,2 & 18,8 & 51,0 \\
\hline $\mathrm{Fev} / 09$ & 1,5 & 32,8 & 79,3 & 102,8 & 5,0 & 29,2 & 20,0 & 47,0 \\
\hline Mar/09 & 1,5 & 30,3 & 79,3 & 92,8 & 11,5 & 28,7 & 20,0 & 46,0 \\
\hline Abr/09 & 1,7 & 30,2 & 73,9 & 74,9 & 9,0 & 23,4 & 14,3 & 50,5 \\
\hline Mai/09 & 2,0 & 39,2 & 89,9 & 73,4 & 9,5 & 22,7 & 13,7 & 52,5 \\
\hline Jun/09 & 2,3 & 38,7 & 86,9 & 56,7 & 9,5 & 19,0 & 10,1 & 52,0 \\
\hline Jul/09 & 2,4 & 36,9 & 83,0 & 44,9 & 8,5 & 19,3 & 11,8 & 64,5 \\
\hline Ago/09 & 2,1 & 40,3 & 86,6 & 76,1 & 8,5 & 22,4 & 12,5 & 46,5 \\
\hline Set/09 & 1,8 & 33,7 & 85,1 & 71,9 & 8,0 & 22,7 & 14,8 & 60,5 \\
\hline Out/09 & 1,5 & 29,5 & 76,4 & 83,4 & 11,5 & 23,5 & 15,2 & 61,5 \\
\hline Nov/09 & 1,4 & 33,2 & 74,0 & 103,2 & 5,5 & 28,4 & 18,6 & 49,0 \\
\hline Dez/09 & 1,5 & 28,7 & 67,9 & 85,6 & 6,5 & 25,9 & 17,8 & 59,5 \\
\hline Jan/10 & 1,7 & 25,0 & 74,1 & 84,4 & 4,5 & 27,4 & 18,6 & 56,0 \\
\hline $\mathrm{Fev} / 10$ & 1,9 & 33,3 & 76,4 & 99,1 & 6,0 & 29,2 & 18,7 & 45,0 \\
\hline Mar/10 & 1,6 & 32,6 & 70,5 & 78,2 & 8,0 & 26,9 & 17,7 & 53,0 \\
\hline Abr/10 & 1,5 & 29,6 & 67,6 & 69,1 & 5,5 & 24,4 & 15,5 & 56,5 \\
\hline Mai/10 & 1,8 & 35,9 & 79,0 & 67,3 & 6,5 & 22,5 & 13,3 & 55,5 \\
\hline Jun/10 & 2,5 & 47,8 & 92,0 & 66,2 & 9,0 & 21,3 & 10,4 & 49,5 \\
\hline Jul/10 & 2,2 & 44,3 & 84,4 & 73,0 & 7,0 & 22,7 & 12,8 & 51,5 \\
\hline $\mathrm{Ago} / 10$ & 2,5 & 58,1 & 106,9 & 97,8 & 8,0 & 19,7 & 11,5 & 64,5 \\
\hline Set/10 & 1,9 & 46,6 & 91,4 & 99,3 & 6,5 & 26,3 & 15,1 & 40,0 \\
\hline Out/10 & 1,3 & 33,1 & 64,1 & 99,5 & 4,5 & 24,9 & 15,3 & $\ldots$ \\
\hline Nov/10 & 1,3 & 30,8 & 59,7 & 97,4 & 5,5 & 26,9 & 17,4 & $\ldots$ \\
\hline Dez/10 & 1,2 & 24,6 & 53,0 & 91,4 & 4,0 & 28,2 & 19,6 & $\ldots$ \\
\hline
\end{tabular}

FONTE: CETESB

NOTA: CO - Monóxido de carbono máximo a cada 8 horas (ppm); MP10 - Material particulado inalável $\left(\mu \mathrm{g} / \mathrm{m}^{3}\right)$; NO2 - Dióxido de nitrogênio $\left(\mu \mathrm{g} / \mathrm{m}^{3}\right)$; O3 - Ozônio $\left(\mu \mathrm{g} / \mathrm{m}^{3}\right)$; SO2 Dióxido de enxofre $\left(\mu \mathrm{g} / \mathrm{m}^{3}\right)$; Tmax - Temperatura máxima $\left({ }^{\circ} \mathrm{C}\right)$; Tmin - Temperatura mínima $\left({ }^{\circ} \mathrm{C}\right)$; UR - Umidade relativa do ar mínima (\%) 
Os dados originais provenientes da CETESB são encontrados no apêndice.

\subsection{Modelagem}

A análise do efeito da lei anti fumo na morbimortalidade por "Infarto" e por "AVC" foi testada utilizando-se modelos de função de transferência controlados pelos fatores ambientais e pelo número total de leitos hospitalares ("leitos"). Foram criados modelos independentes para "Infarto" e para "AVC", bem como para internações e mortalidade.

\subsubsection{Modelo ARIMAX internação por "Infarto"}

O modelo estimado para taxa mensal de internações por "Infarto" na rede hospitalar do SUS foi ajustado para número de leitos hospitalares SUS, umidade relativa do ar mínima, temperatura mínima e concentração máxima de monóxido de carbono, conforme Equação (1).

$$
\begin{gathered}
\text { TX }_{\text {Infarto }}=0,0031 \text { LEITOS }_{t-0}-0,1741 \text { MIN }_{t-0}+0,4015 \text { URMIN }_{t-7}+0,3151 \text { COMAX }_{t-5} \\
-0,7816 \text { LEI }_{t-0}+0,8296 \text { LEI I }_{t-3}+\left(1+0,4602 B^{14}\right) \text { at }
\end{gathered}
$$

Neste modelo, observou-se que a intervenção teve um efeito instantâneo sobre a série "taxa de internação por infarto", ou seja, à partir do momento em que a lei entrou em vigor, o efeito foi imediatamente sentido, com uma redução de 0,78 casos de "Infarto" por mês por 100000 usuários SUS. Esse efeito foi verificado no 
Resultados

mês em que a lei entrou em vigor e persistiu por 2 meses após, totalizando 3 meses de efeito (Figura 1, Anexo A), com uma queda de $5,4 \%$ ao mês ( $p=0,022$; IC95\% -1,45- -0,11). Em números absolutos, estima-se que foram evitados 142 casos de "Infarto" nos 3 meses iniciais da vigência da lei (agosto a outubro de 2009). Posteriormente a esse período, a curva retornou ao seu nível anterior (prélei).

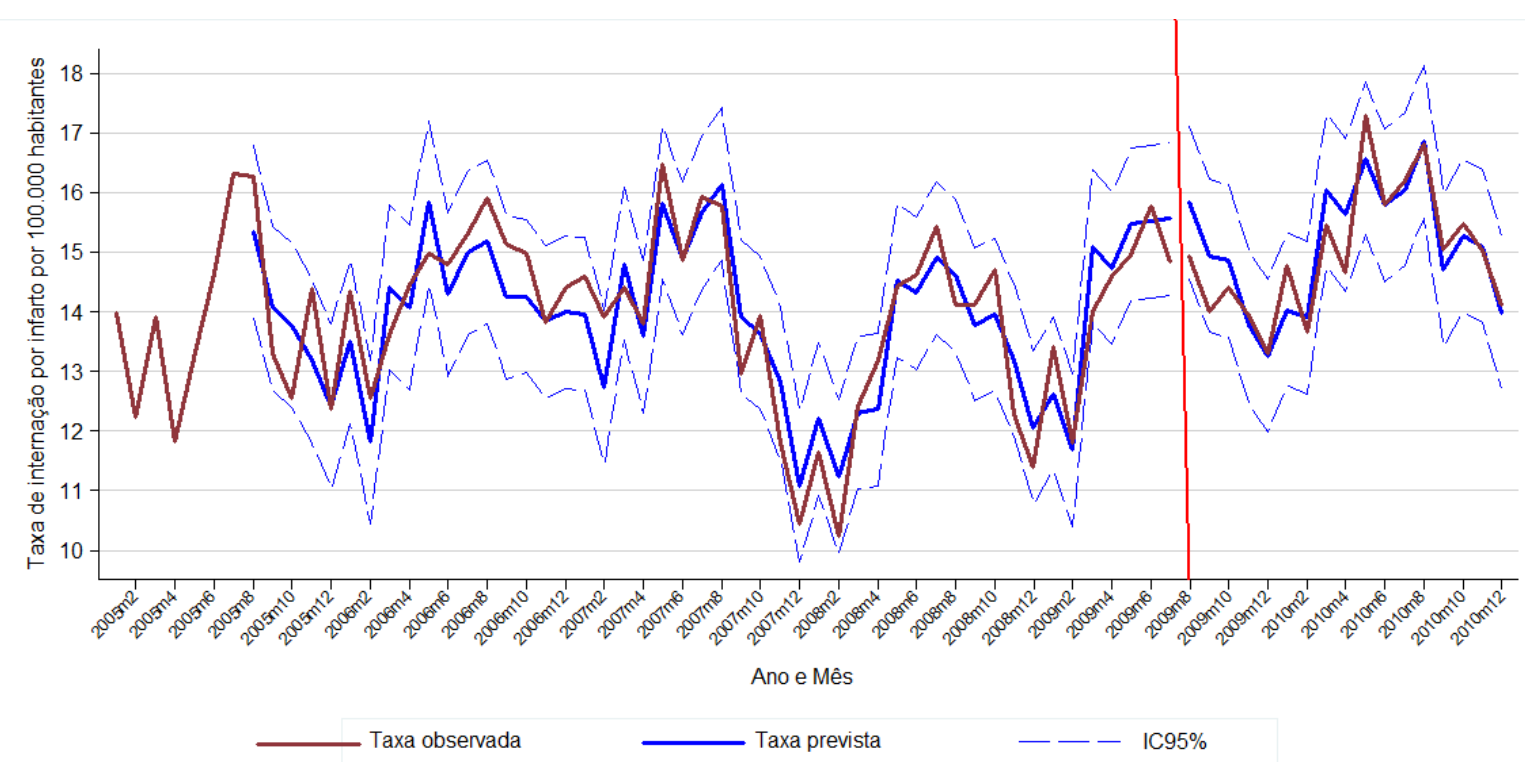

Figura 1 - Taxa observada de internações por "Infarto" e taxa prevista ${ }^{* *}$ de internações por "Infarto", rede hospitalar SUS, Município de São Paulo - 2005 a 2010, IC 95\%, modelo ARIMAX

*por 100000 habitantes

**ajustada por número de leitos hospitalares SUS, URmin, Tmin, CO

\subsubsection{Modelo ARIMAX internação por "AVC"}

O modelo estimado para taxa mensal de internações por "AVC" na rede hospitalar do SUS foi ajustado para número de leitos hospitalares SUS, 
Resultados

temperatura mínima e concentração máxima de monóxido de carbono, conforme Equação (2).

$$
\begin{gathered}
T x_{A V C}=0,00022 \text { LEITOS }_{t-0}-0,1503 \text { TMIN }_{t-0}+0,7250 \operatorname{COMAX}_{t-5}-0,7202 L E I_{t-7} \\
+1,019 L E I_{t-13}+\left(1+0,2821 B^{7}\right) a t
\end{gathered}
$$

Neste modelo, observou-se efeito após 7 meses da intervenção (Lag 7), com uma redução de 0,72 casos de "AVC" por mês por 100000 usuários SUS. Esse efeito persistiu até 12 meses após a intervenção (Figura 2, Anexo B), com uma queda de $7,4 \%$ ao mês entre o $7^{\circ}$ e o $12^{\circ}$ meses $(p=0,012 ;$ IC95\% $-1,28$ - 0,16) . Em números absolutos, estima-se que foram evitados 255 casos de "AVC" entre $07^{\circ}$ e $012^{\circ}$ meses após a lei anti fumo.

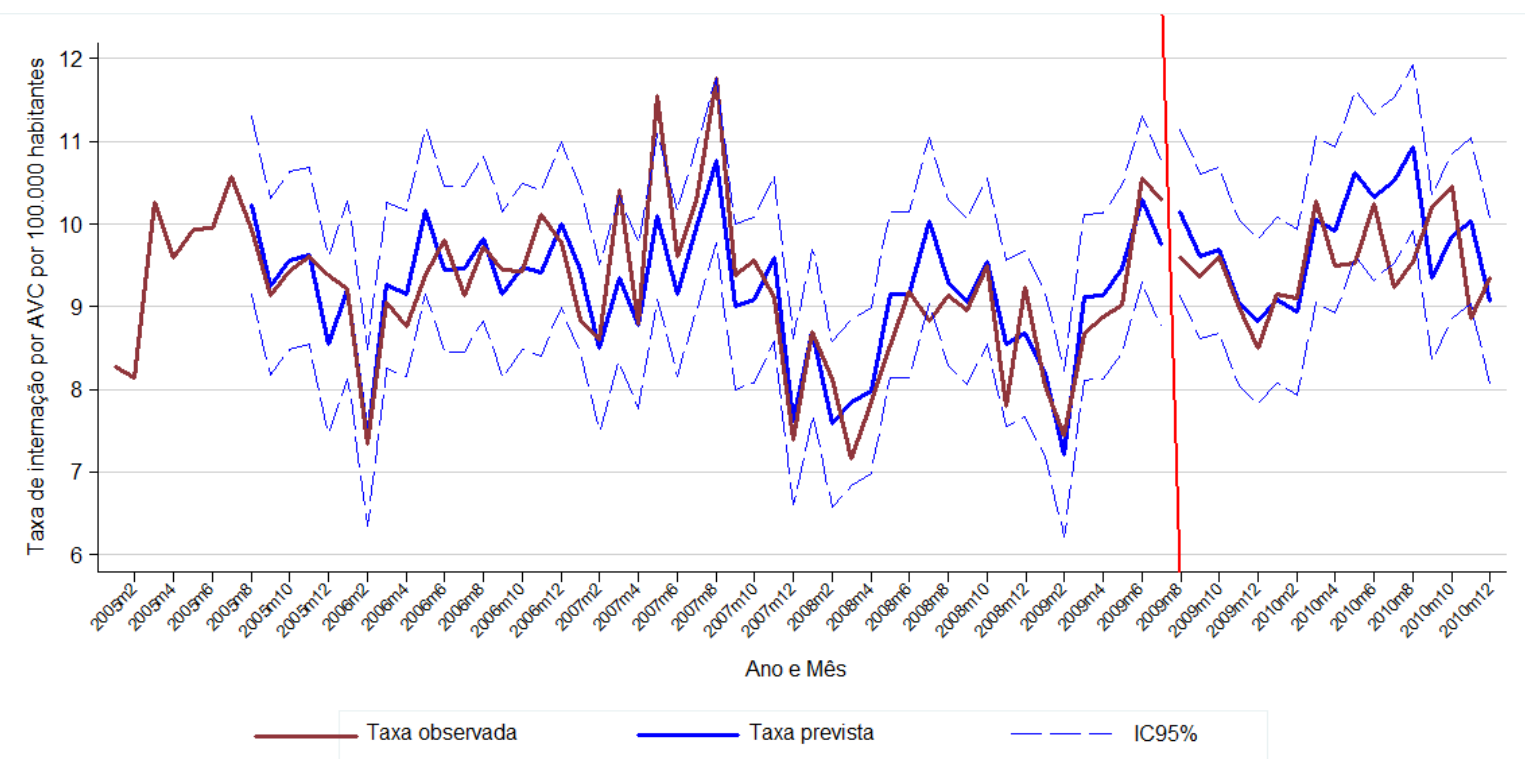

Figura 2 - Taxa observada de internações por "AVC" e taxa prevista ${ }^{* *}$ de internações por "AVC", rede hospitalar SUS, Município de São Paulo - 2005 a 2010, IC 95\%, modelo ARIMAX 
*por 100000 habitantes

**ajustada por número de leitos hospitalares SUS, Tmin, CO

\subsubsection{Modelo ARIMAX mortalidade por "Infarto"}

O modelo estimado para taxa mensal de mortalidade por "Infarto" foi ajustado para número de leitos hospitalares SUS, temperatura mínima e concentração máxima de monóxido de carbono, conforme Equação (3).

Mort $_{\text {Infarto }}=0,00012$ LEITOS $_{t-0}-0,0856$ TMIN $_{t-0}+0,2398$ COMAX $_{t-5}-0,5245 L E I_{t-0}$

Neste modelo, observou-se que a intervenção teve efeito imediato sobre a taxa de mortalidade, com uma redução de 0,94 casos por mês por 100000 habitantes. Esse efeito foi persistente por todo o período de estudo (Figura 3, Anexo C), com uma queda de $11,9 \%$ ao mês $(p<0,001 ;$ IC95\% $-1,34--0,54)$. Em números absolutos, estima-se que foram evitados 571 óbitos por "Infarto" durante os 17 meses analisados após a lei anti fumo. 


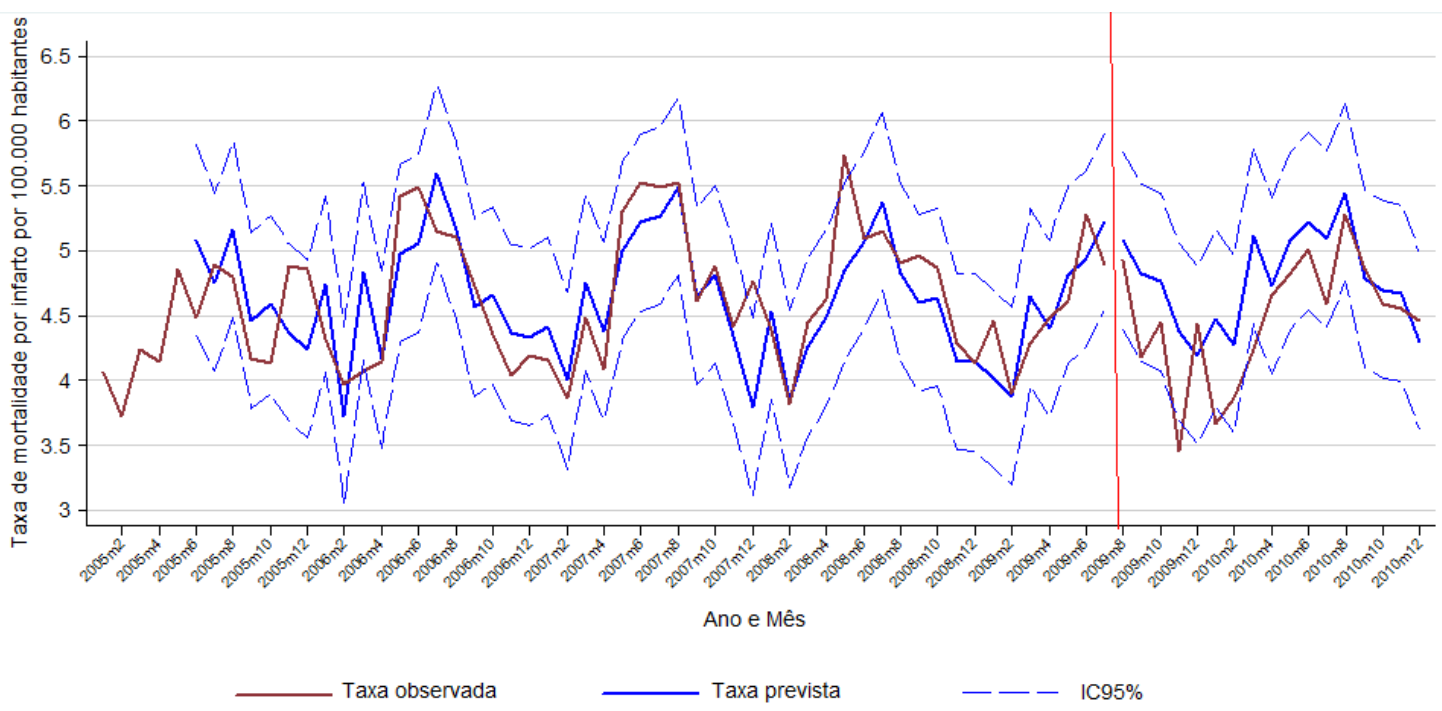

Figura 3 - Taxa observada de mortalidade por "Infarto" e taxa prevista ${ }^{* *}$ de mortalidade por "Infarto", Município de São Paulo - 2005 a 2010, IC 95\%, modelo ARIMAX

*por 100000 habitantes

**ajustada por número de leitos hospitalares SUS, Tmin, CO

\subsubsection{Modelo ARIMAX mortalidade por "AVC"}

O modelo estimado para taxa mensal de mortalidade por "AVC" foi ajustado para número de leitos hospitalares SUS, temperatura mínima e concentração máxima de monóxido de carbono, conforme Equação (4).

$$
\begin{gathered}
\text { Mort }_{\text {AVC }}=0,00003 \text { LEITOS }_{t-0}-0,0391 \text { MIN }_{t-0}+0,1053 \text { COMAX }_{t-6} \\
+0,9951 \text { CIDJJNON }_{t-0}-0,1109 \text { LEI I }_{t-0}
\end{gathered}
$$

Neste modelo, observou-se que a intervenção teve efeito imediato sobre a taxa de mortalidade (Lag 0), com uma redução de 0,11 casos por mês por 100000 
habitantes. Esse efeito foi persistente por todo o período de estudo (Figura 4, Anexo D), com uma queda de 5,3\% ao mês ( $p=0,031 ; \mathrm{IC} 95 \%-0,21--0,01)$. Em números absolutos, estima-se que foram evitados 228 óbitos por "AVC" nos 17 meses iniciais após a lei anti fumo.

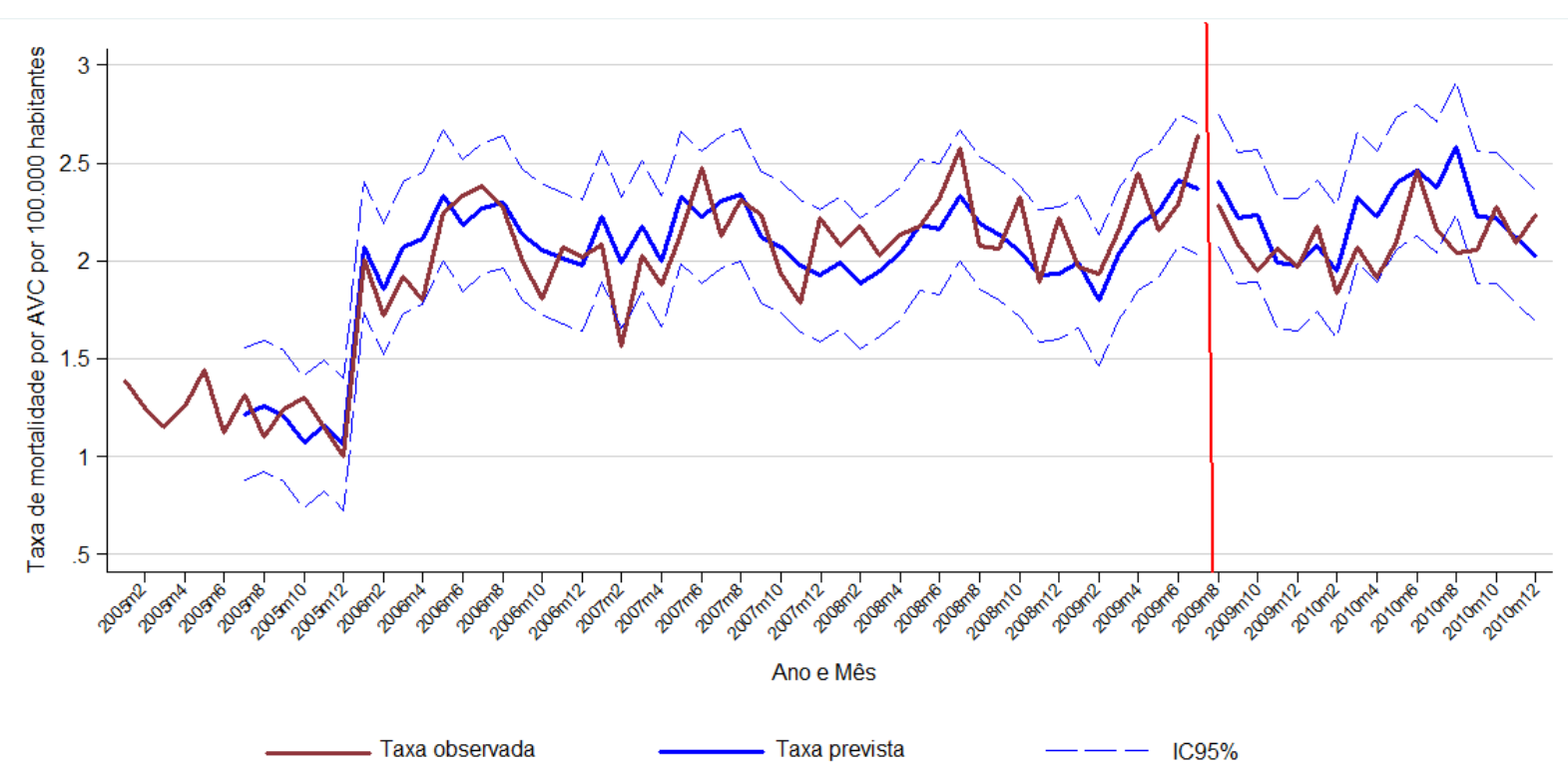

Figura 4 - Taxa observada de mortalidade por "AVC" e taxa prevista" de mortalidade por "AVC", Município de São Paulo - 2005 a 2010. IC 95\%, modelo ARIMAX

*por 100000 habitantes

**ajustada por número de leitos hospitalares SUS, Tmin, CO

\subsubsection{Modelo ITSA-ARIMAX internação por "Infarto"}

O modelo estimado para taxa mensal de internações por "Infarto" na rede hospitalar do SUS foi ajustado para número de leitos hospitalares SUS, umidade relativa mínima, temperatura mínima e concentração máxima de monóxido de carbono. Neste modelo, observou-se que a série taxa de internações por infarto 
Resultados

apresentava tendência decrescente, com uma redução mensal de 0,016 casos por 100000 usuários SUS, antes da publicação da lei anti fumo $(p=0,003)$. Após a publicação da lei (intervenção), essa tendência foi perdida $(\beta=0,43, p=0,21)$, com a série estabilizando-se em torno do nível médio, o qual não sofreu nenhum efeito significativo devido à intervenção (Figura 5, Anexo E).

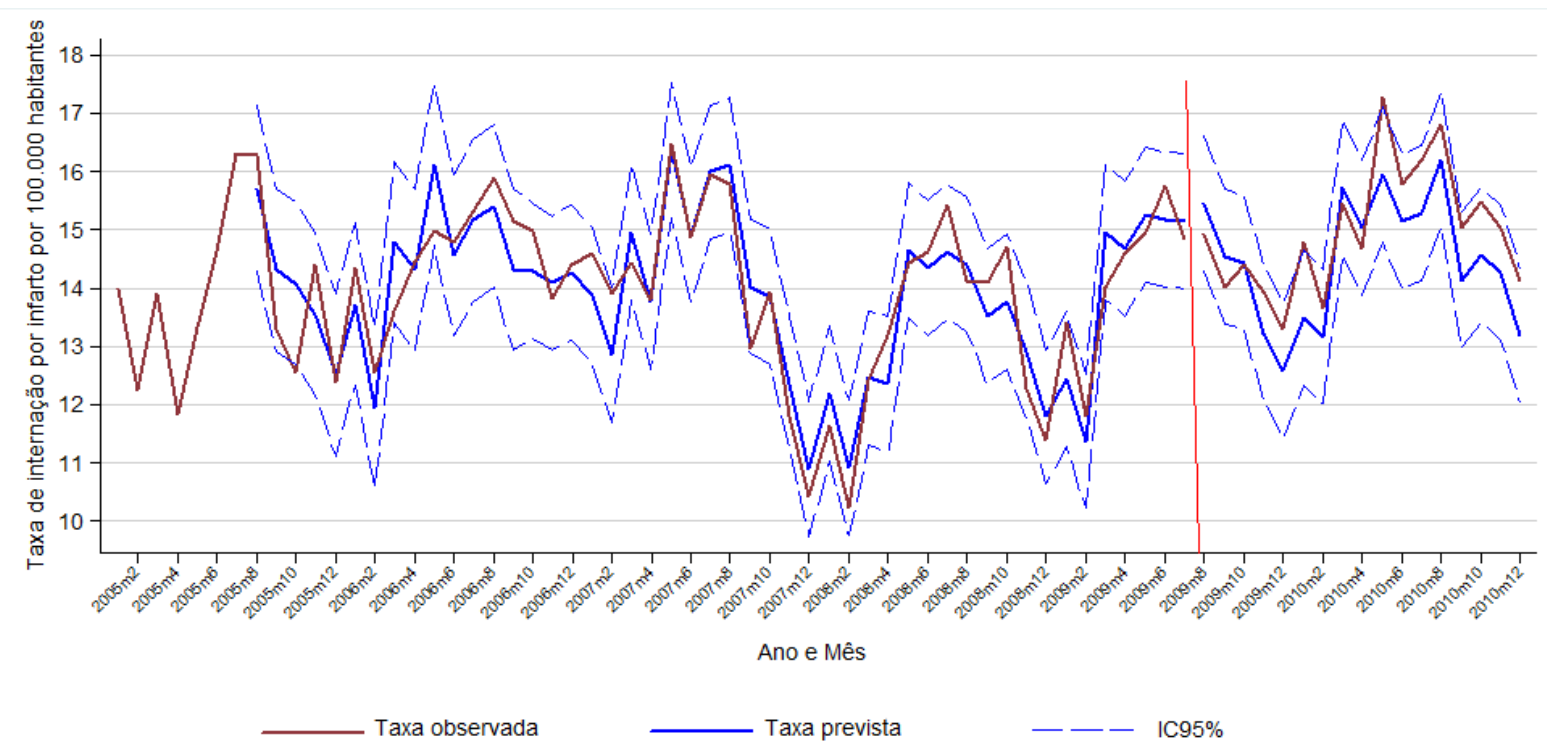

Figura 5 - Taxa observada de internações por "Infarto" e taxa prevista ${ }^{* *}$ de internações por "Infarto", rede hospitalar SUS, Município de São Paulo - 2005 a 2010, IC 95\%, modelo ITSA-ARIMAX

*por 100000 habitantes

**ajustada por número de leitos hospitalares SUS, URmin, Tmin, CO

\subsubsection{Modelo ITSA-ARIMAX internação por "AVC"}

O modelo estimado para taxa mensal de internações por "AVC" na rede hospitalar do SUS foi ajustado para número de leitos hospitalares SUS, umidade relativa mínima, temperatura mínima e concentração máxima de monóxido de carbono. Neste modelo, observou-se que a série taxa de internações por AVC 
Resultados

apresentava tendência decrescente, com uma redução média de 0,009 casos por 100000 usuários SUS, antes da publicação da lei anti fumo $(p=0,015)$ e que, após a publicação da lei (intervenção), essa tendência foi perdida ( $\beta=0,86, p=0,041$ ). Observou-se uma mudança de nível no Lag 8 após a intervenção, com redução de 1,2 casos por mês por 100000 usuários SUS $(p=0,024)$ (Figura 6, Anexo F).

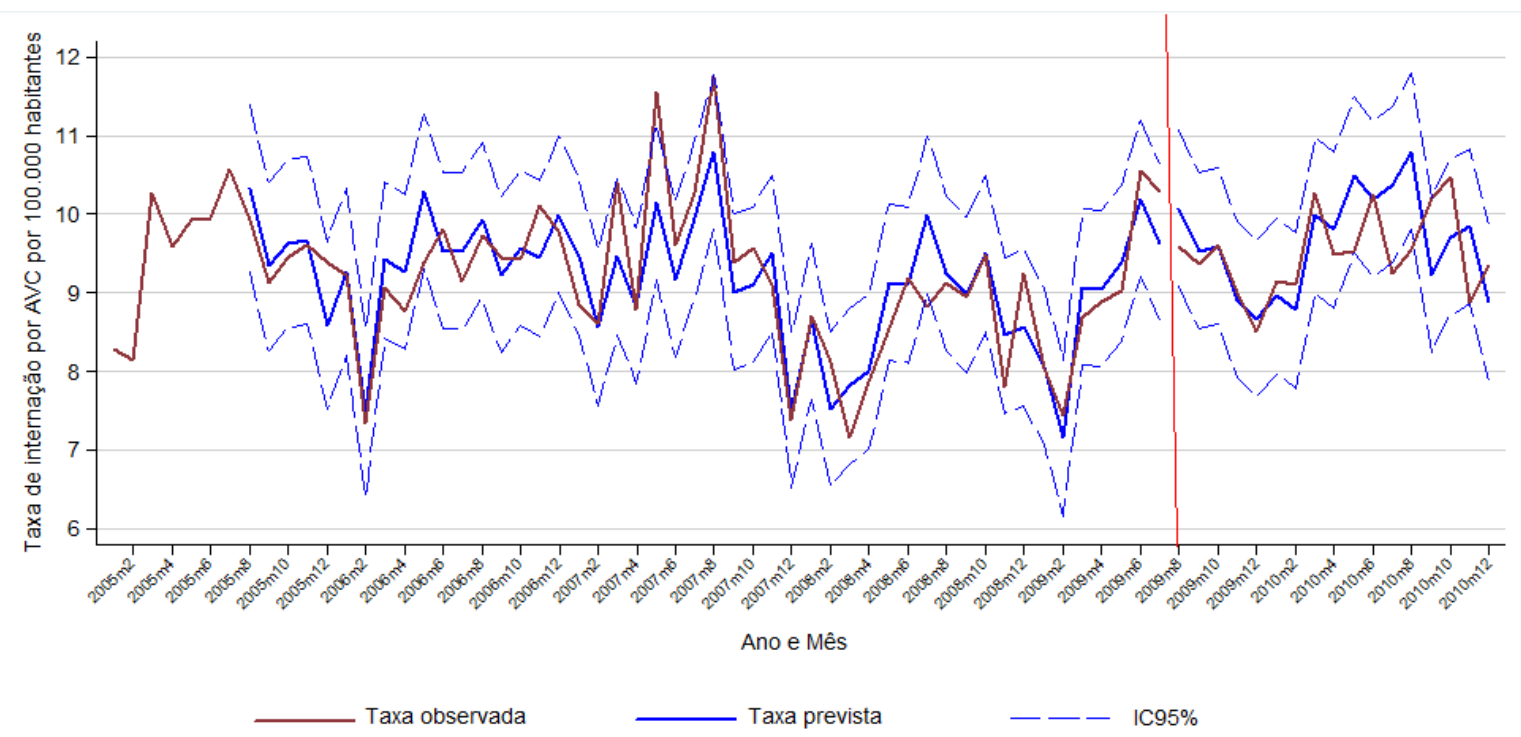

Figura 6 - Taxa observada de internações por "AVC" e taxa prevista" de internações por "AVC", rede hospitalar SUS, Município de São Paulo - 2005 a 2010, IC 95\%, modelo ITSA-ARIMAX

*por 100000 habitantes

**ajustada por número de leitos hospitalares SUS, URmin, Tmin, CO

\subsubsection{Modelo ITSA-ARIMAX mortalidade por "Infarto"}

O modelo estimado para taxa mensal de mortalidade por "Infarto" foi ajustado para número de leitos hospitalares SUS, temperatura mínima e concentração máxima de monóxido de carbono. Neste modelo, observou-se que a 
Resultados

série taxa de mortalidade por infarto não apresentava tendência $(\beta=0,12, p=0,07)$ antes da publicação da lei anti fumo e que, após a publicação da lei (intervenção), permaneceu constante $(\beta=0,35, p=0,294)$. Observou-se mudança imediata do nível da série após a intervenção, com uma redução mensal de 1,6 casos por 100000 habitantes ( $p=0,024)$ (Figura 7, Anexo G).

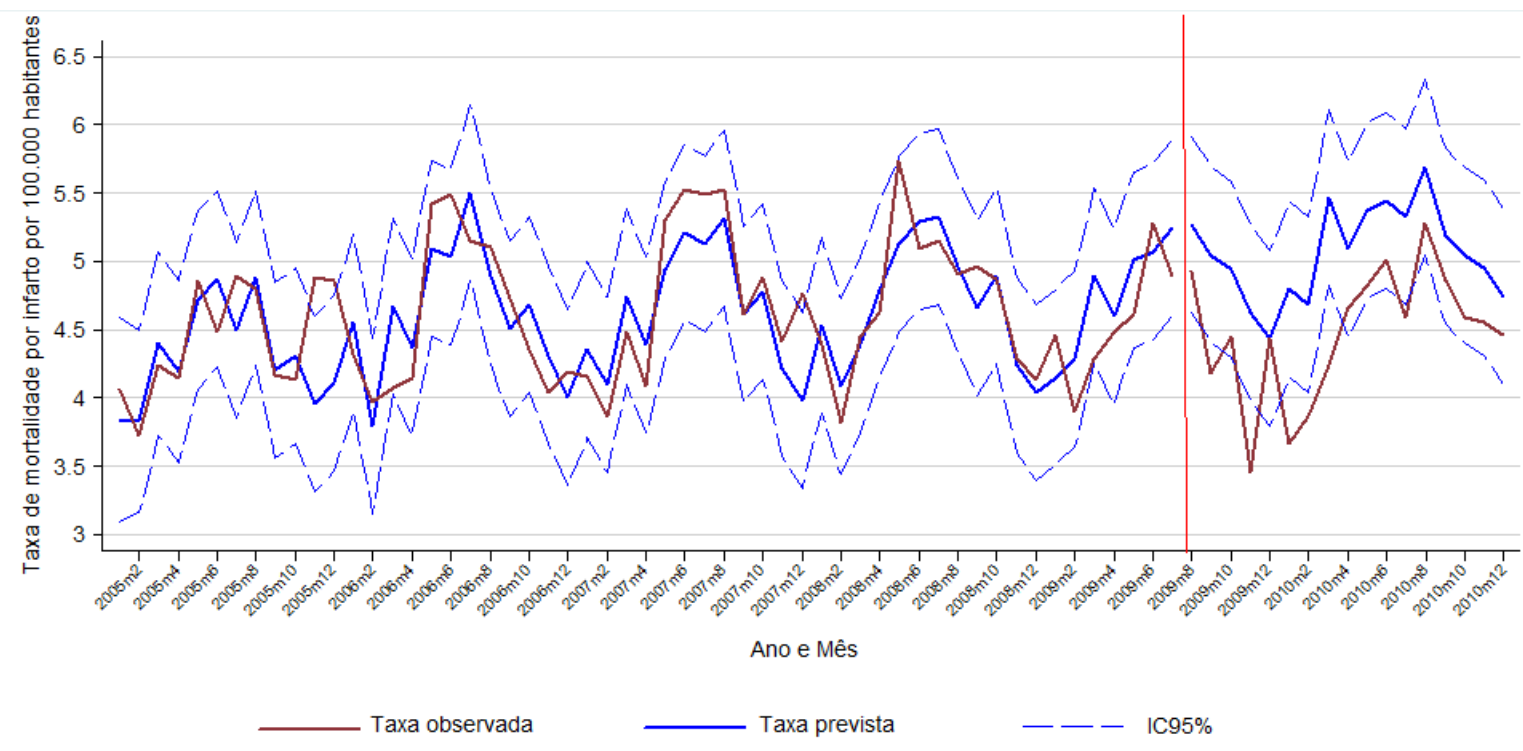

Figura 7 - Taxa observada de mortalidade por "Infarto" e taxa prevista ${ }^{* *}$ de mortalidade por "Infarto", Município de São Paulo - 2005 a 2010, IC 95\%, modelo ITSA-ARIMAX

*por 100000 habitantes

**ajustada por número de leitos hospitalares SUS, Tmin, CO

\subsubsection{Modelo ITSA-ARIMAX mortalidade por "AVC"}

O modelo estimado para taxa mensal de mortalidade por "AVC" foi ajustado para número de leitos hospitalares SUS, umidade relativa mínima, temperatura mínima e concentração máxima de monóxido de carbono. Neste modelo, observou-se que a série taxa de mortalidade por AVC não apresentava tendência 
Resultados

( $\beta=0,12, p=0,07$ ) antes da publicação da lei anti fumo e que, após a publicação da lei (intervenção), a série manteve-se constante $(\beta=0,30, p=0,283)$. Observouse mudança de nível imediata após a intervenção, com uma redução mensal de $0,8$ casos por 100000 habitantes $(p=0,006)$ (Figura 8 , Anexo $H)$.

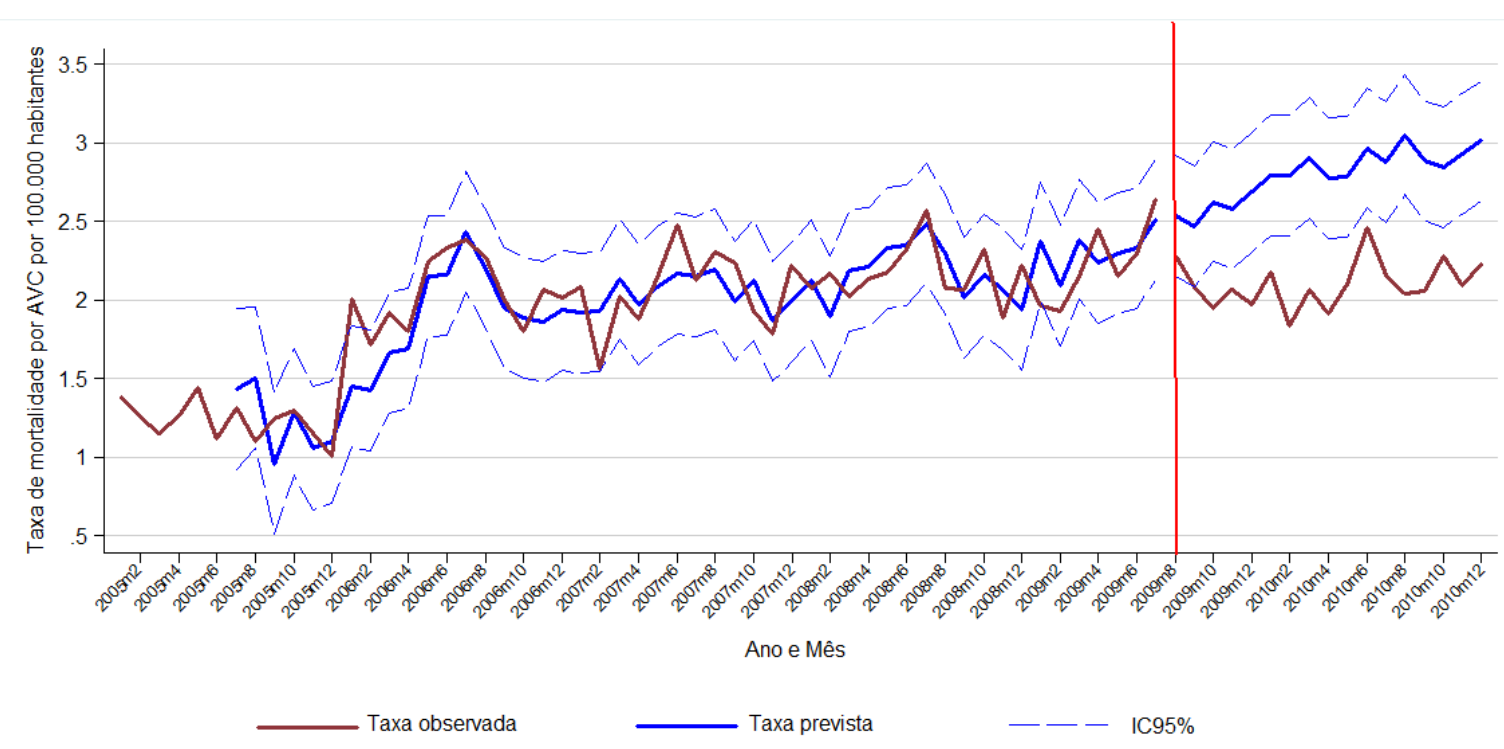

Figura 8 - Taxa observada de mortalidade por "AVC" e taxa prevista ${ }^{* *}$ de mortalidade por "AVC", Município de São Paulo - 2005 a 2010, IC 95\%, modelo ITSA-ARIMAX

*por 100000 habitantes

**ajustada por número de leitos hospitalares SUS, URmin, Tmin, CO 


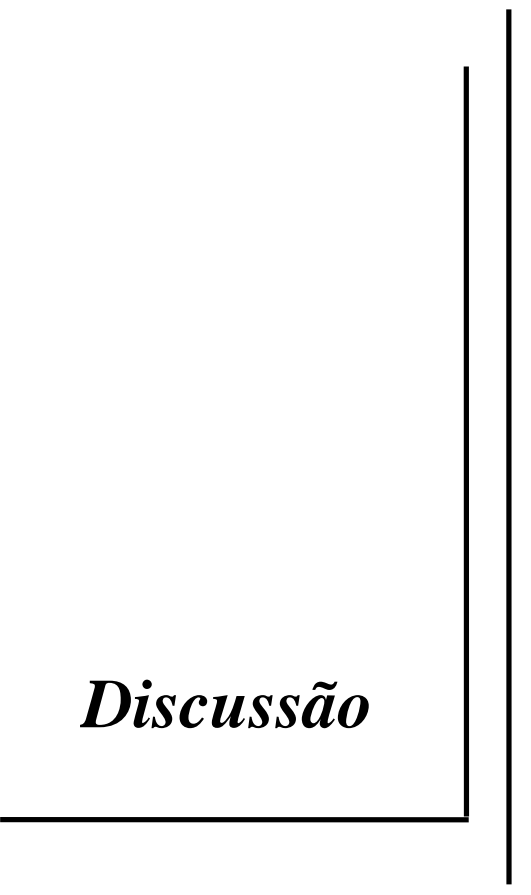




\section{DISCUSSÃO}

Este estudo epidemiológico buscou analisar se as taxas de internação e de mortalidade por doenças cardiovasculares e cerebrovasculares sofreram influência da lei antifumo, bem como se interferentes ambientais e número de leitos hospitalares disponíveis influenciaram a ocorrência destas taxas.

Em nosso resultado, encontramos uma redução nas taxas de mortalidade e internação, tanto por "Infarto" quanto por "AVC". A redução nas taxas de mortalidade por "Infarto" e por "AVC" foi muito mais expressiva que a redução nas taxas de internação hospitalar pelas mesmas causas, além de ter um efeito mais duradouro após a lei anti fumo. Uma das explicações para esta diferença baseiase no fato de que o dado de mortalidade é mais completo e menos sujeito a erros que o dado de internações hospitalares. Além disso, a taxa mortalidade calculada contempla toda a população do município de São Paulo, ao passo que a taxa de internação refere-se somente à população coberta exclusivamente pelo Sistema Único de Saúde (SUS), ou seja, cerca de 55\% da população do município de São Paulo. Os demais $45 \%$ da população são atendidos pelo sistema suplementar de saúde, cujos dados não se encontram disponíveis para consulta, não são padronizados e nem controlados em sua aquisição.

Analisando estudos prévios realizados para a avaliação da influência das leis anti fumo na incidência de internações e mortalidade por infarto agudo do miocárdio, nota-se uma redução nas taxas de internação na maior parte dos 
estudos $^{28,34,36,42,60,71,72}$ e uma redução na mortalidade em uma parte dos estudos. $^{73-76}$ Esta redução é verificada de maneira mais significativa no primeiro ano após o início da vigência das leis, sendo que a maior parte dos estudos avaliou períodos de 12 a 24 meses após o início da vigência de suas leis. Além disso, observa-se que estudos realizados em cidades ou localidades menores, em que é possível realizar um controle da população e dos locais de atendimeto médico, o efeito da lei foi mais exuberante, ${ }^{34,42}$ ao passo que em localidades maiores ou países os efeitos da lei são pequenos ou moderados. ${ }^{35,71}$ Estudos que avaliaram a influência da lei anti fumo e AVCs são esparsos na literatura e tem resultados divergentes. ${ }^{72,77}$

Por outro lado, nenhum estudo encontrado na literatura avaliou conjuntamente variáveis biológicas, como o monóxido de carbono, durante este processo em amostra de magnitude semelhante à nossa. O fato de termos observado uma redução significativa na concentração de monóxido de carbono em locais como bares e restaurantes comprovou a obediência imediata à lei e um provável benefício para a população em geral e principalmente para os tabagistas passivos.

Inúmeros estudos encontraram associação significativa entre níveis de monóxido de carbono, material particulado inalável (com diâmetro $\leq 10 \mu \mathrm{m})$, ozônio e outros poluentes e uma série de efeitos deletérios à saúde da população exposta a estes poluentes, que vão desde sintomas respiratórios, cefaléia, tontura, sintomas cardiovasculares, até aumento da taxa de mortalidade na população. ${ }^{78-81}$ 
Estudo realizado no Brasil com doenças do aparelho circulatório e, mais especificamente, doenças isquêmicas do coração encontrou associações estatisticamente significantes para todos os poluentes, com exceção do O3. Um aumento de $10 \mu \mathrm{g} / \mathrm{m} 3$ nos níveis de PM10 esteve associado a um incremento nas hospitalizações em idosos por doenças do aparelho circulatório de 1\% e de 1,5\% para doenças isquêmicas do coração. ${ }^{82}$

Entretanto, apesar de existirem inúmeros estudos avaliando leis antifumo e taxas de internação e diversos outros estudos avaliando a influência de poluentes ambientais com a mobimortalidade por doenças cardiovasculares, poucos estudos avaliaram em conjunto todas estas variáveis.

No presente estudo, houve uma redução do nível de monóxido de carbono em ambientes coletivos fechados onde foi proibido o fumo. Porém, não era esperada uma alteração nos níveis de monóxido de carbono e outros poluentes na atmosfera (ambientes abertos) somente em virtude da lei, uma vez que estes poluentes tendem a ficar concentrados em ambientes fechados onde há pessoas que fumam, mas se dispersam rapidamente em ambientes abertos.

A inclusão de dados ambientais na análise foi realizada para se excluir a possibilidade de que alterações nos níveis destes poluentes pudessem ser as responsáveis pelo resultado obtido. Esta hipótese foi levada em consideração, pois ao longo destes 6 anos analisados no estudo foram introduzidas algumas medidas governamentais para a redução da poluição ambiental, sobretudo aquela gerada pela emissão de poluentes por veículos automotores. Em 1997 foi introduzido o rodízio municipal de veículos, em que veículos particulares e de 
Discussão

empresas de qualquer cidade, excetuando-se aqueles que realizam funções essenciais, transporte urbano e escolar, atendimento médico, transporte de produtos perecíveis, veículos dirigidos por pessoas portadoras de deficiência física ou que transportem estas últimas, são impedidos de circular no centro expandido da capital paulista uma vez por semana, segundo uma escala pré estabelecida, em duas faixas de horário do dia: das 7 às 10 horas e das 17 às 20 horas. Neste mesmo ano, o uso dos catalisadores de veículos se tornou obrigatório. Mais recentemente, a inspeção veicular ambiental foi introduzida também com o intuito principal de reduzir a emissão de poluentes na atmosfera.

A inspeção veicular teve início na cidade de São Paulo em 1962, sofreu diversas modificações ao longo dos anos e vem sendo realizado pela Prefeitura do município de São Paulo de maneira mais estruturada desde 2008, quando iniciou a inspeção da frota a diesel registrada na cidade. Em 2009 passaram pela inspeção todos os veículos a diesel, as motos e os carros movidos a álcool, gás natural ou gasolina registrados na cidade de São Paulo entre 2003 e 2008. Em 2010, o programa atingiu $100 \%$ da frota (6,5 milhões de veículos).

Com relação a mudanças no tratamento do infarto agudo do miocárdio e do acidente vascular cerebral, houve o início de um importante protocolo para aprimorar o tratamento do infarto agudo do miocárdio em hospitais públicos da cidade de São Paulo. Coordenado pela Sociedade de Cardiologia do Estado de São Paulo (SOCESP), o "Projeto Infarto" teve início em abril de 2010, em uma ação conjunta da SOCESP, da Secretaria Municipal de Saúde e da Secretaria Estadual de Saúde, além do Hospital São Paulo da Universidade Federal de São 
Paulo. A principal finalidade deste projeto foi a melhoria no atendimento inicial ao paciente com infarto agudo do miocárdio, visando à redução da mortalidade por esta causa. Durante o ano de 2010, foi realizado um piloto do projeto em 5 hospitais da periferia de São Paulo. A primeira reunião do grupo ocorreu em abril de 2010 e as palestras e treinamentos nos 5 hospitais iniciaram em maio de 2010. Os hospitais englobados no projeto piloto foram: Hospital Geral do Grajau, Hospital do Tatuapé, Hospital Tide Setubal (ou Hospital de São Miguel Paulista), Hospital Artur Ribeiro de Saboya (ou Hospital do Jabaquara) e Hospital Doutor José Soares Hungria (ou Hospital de Pirituba). Paralelamente a esses treinamentos, foi disponibilizado na rede pública do município de São Paulo o trombolítico Tenecteplase, um importante medicamento no tratamento do infarto agudo do miocárdio com supradesnivelamento do segmento ST, principalmente em hospitais que não possuem serviço de radiologia intervencionista cardíaca (serviço de hemodinâmica) disponível 24 horas por dia.

No ano de 2011, o projeto foi expandido para outros hospitais da cidade de São Paulo e, em 2014, foi estendido para o interior do estado. Uma vez que o período de análise de nosso estudo termina em dezembro de 2010, acreditamos que não houve influência deste Projeto nos resultados que encontramos (encontrava-se na fase piloto do projeto).

Outras modificações, seja no atendimento, seja na utilização de medicações para infarto e AVC, não foram encontradas durante o levantamento realizado. As principais diretrizes assistenciais não tiveram modificações 
importantes entre os anos de 2009 e 2010 que pudessem contribuir para os resultados obtidos.

Em relação ao número de tabagistas ativos, a Vigilância de Fatores de Risco e Proteção para Doenças Crônicas por Inquérito Telefônico (VIGITEL) mostrou que, no município de São Paulo, a porcentagem de tabagistas ativos oscilou entre 18 e $21 \%$ entre os anos de 2006 e $2011,{ }^{83-87}$ ou seja, não houve uma queda expressiva de fumantes ativos durante este período (Tabela 8).

Tabela 8: Percentual de tabagistas segundo a VIGITEL, Município de São Paulo 2006 a 2011

\begin{tabular}{cc}
\hline ANO & TOTAL (\%) \\
\hline 2006 & 18,8 \\
2007 & 21,7 \\
2008 & 21 \\
2009 & 18,8 \\
2010 & 19,6 \\
2011 & 19,3 \\
\hline
\end{tabular}

FONTE: VIGITEL

\subsection{Limitações do estudo}

Trata-se de estudo ecológico de séries temporais em que houve uma intervenção populacional legislativa em determinado ponto da série. De acordo com este desenho, não é possível estabelecer relação de causalidade entre os eventos descritos e, por este motivo, foram pesquisados outros fatores que 
Discussão

poderiam interferir da mesma maneira nas séries. Não foram encontrados outros fatores que explicassem as mudanças nas séries.

Avaliando-se a proposição inicial do trabalho, também encontramos uma dificuldade muito grande em obter dados do sistema de saúde suplementar, para compor a totalidade da cobertura hospitalar no município de São Paulo. Devido a isso, os dados de morbidade (internações hospitalares) foram analisados somente para a parcela da população atendida pelo SUS. 


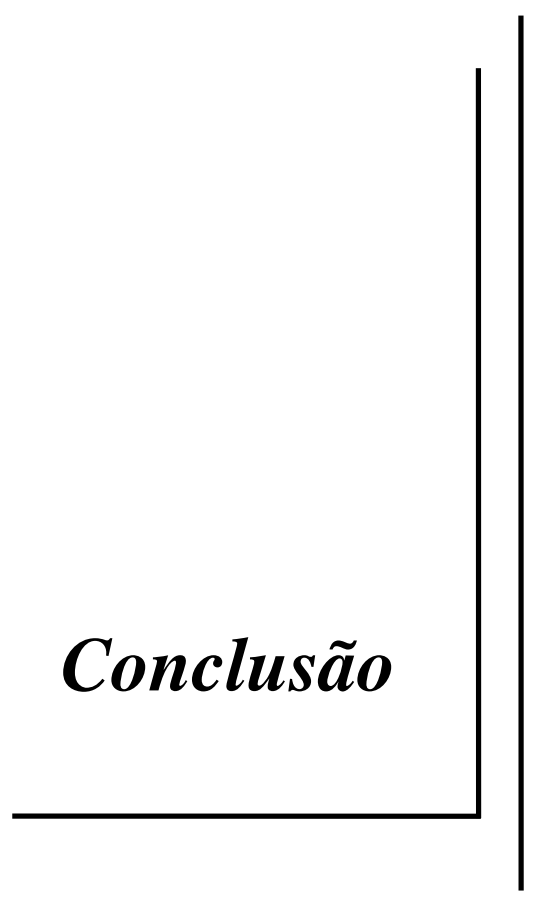




\section{CONCLUSÃO}

Após a lei anti fumo foram observadas:

i. Redução nas taxas de internação por "Infarto" nos 3 meses iniciais

ii. Redução nas taxas de internação por "AVC" entre o $7^{\circ}$ e o $12^{\circ}$ meses

iii. Redução nas taxas de mortalidade por "Infarto" por todo o período estudado

iv. Redução nas taxas de mortalidade por "AVC" por todo o período estudado 


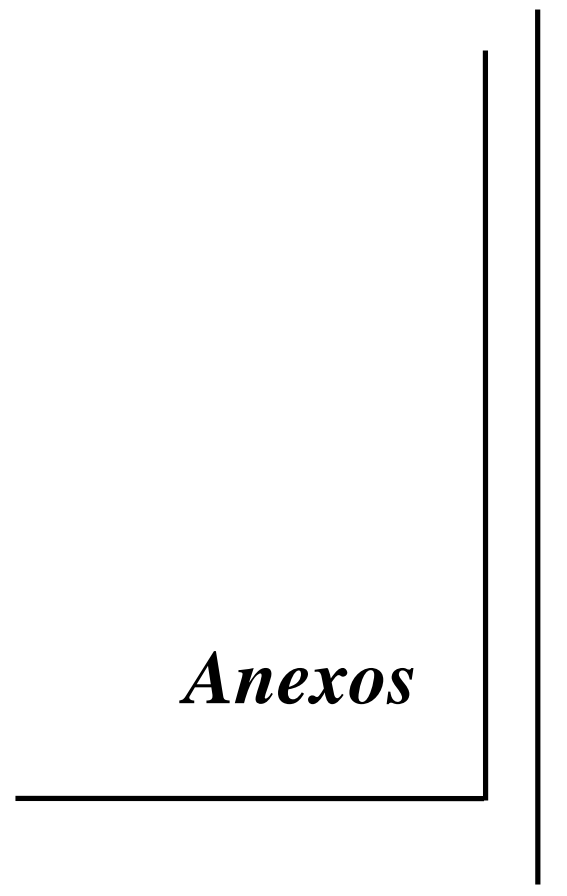




\section{ANEXOS}

\section{Anexo A: Modelo estimado para taxa de internações por "Infarto", metodologia}

ARIMAX

Modelol - Completo Monday December 21 11:32:25 2015 Page 1

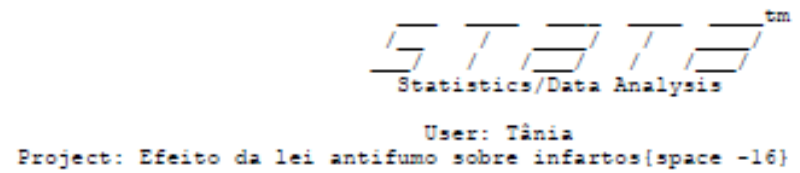

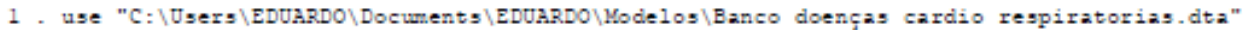

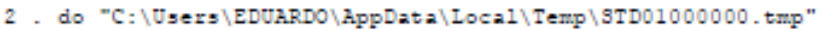

3. arima infartots $1(0)$. leitos $1(0)$. tmin $1(7)$.urmenin $1(5)$. comax $1\left(\begin{array}{ll}0 & 3\end{array}\right)$. intervencao, ar (14) nocon

Iteration $0: \quad \log$ likelihood $=-60.579963$

Iteration 1: log likelihood $=-60.553877$

$\log$ likelihood $=-60.502909$

Iteration 3: $\log$ likelihood $=-60.432879$

Iteration 4: $\log$ likelihood $=-60.379826$

Iteration 5: $\log$ likelihood $=-60.37964$

Iteration 6: $\log$ likelihood $=-60.376678$

Iteration 7: log likelihood $=-60.295336$

Iteration 8: log likelihood $=-60.274951$

Iteration 9: log likelihood $=-60.239848$

BFGS stepping has contracted, resetting BFGS Hessian (0)

Iteration 10: log likelihood $=-60.226185$

Iteration 11: log likelihood $=-60.226153$ (backed up)

Iteration 12: $\log$ likelihood $=-60.225934$ (backed up)

Iteration 13: log likelihood $=-60.224695$ (backed up)

Itceration 14: log likelihood $=-60.224643$ (backed up)

(backed up)

log likelihood $=-60.223857$

Iteration 16: log likelihood $=-60.223842$

teration 17: log likelihood $=-60.22384$

Iteration 18: $\log$ likelihood $=-60.223768$

BFGS stepping has contracted, resetting BFGS Hessian (1)

Iteration 19: log likelihood $=-60.223766$

Iteration 20: $\log$ likelihood $=-60.223766$ (backed up)

Iteration 21: log likelihood $=-60.223766$ (backed up)

Iteration 22: log likelihood $=-60.223766$ (backed up)

Tteration 23: $\log$ likelihod $=-60.223765$ (backed up)

(backed up

Iteration 24: log likelihood $=-60.22376$ (backed up)

Iteration 25: log likelikood $=-60.22375$

teration 26: $\log$ likelihood $=-60.223756$

ARIMA regression

Sample: $2005 n 8-2010 \mathrm{~m} 12$

$\begin{array}{llr}\text { Nurber of obs } & = & 65 \\ \text { Nald chi2 }(7) & = & 68150.80\end{array}$

Log likelihood $=-60.22376$

Prob>chi2 $\quad 0.0000$

\begin{tabular}{|c|c|c|c|c|c|c|}
\hline infartotx & Coef. & OIM & $z$ & P> $>1$ & [95: Conf. & Intervall \\
\hline \multicolumn{7}{|l|}{ infartotx } \\
\hline leitos & .0003151 & .0000148 & 21.32 & 0.000 & .0002861 & .000344 \\
\hline tmin & -.1740876 & .0277798 & -6.27 & 0.000 & -.2285351 & -.1196401 \\
\hline I7. & .040148 & .0125655 & 3.20 & 0.001 & .0155201 & .064776 \\
\hline $\begin{array}{r}\text { comax } \\
\text { I5 }\end{array}$ & 3150745 & 1285895 & 2.45 & 0.014 & 0630438 & .5671053 \\
\hline intervencao & & & & & & \\
\hline- & -.7816501 & .3418954 & -2.29 & 0.022 & -1.451753 & -.1115474 \\
\hline L3. & .8296034 & .3956082 & 2.10 & 0.036 & .0542256 & 1.604981 \\
\hline \multicolumn{7}{|l|}{ ARUA } \\
\hline 214. & -.4602325 & .1150364 & -4.00 & 0.000 & -.6856998 & -.2347652 \\
\hline /signa & .5956811 & .0529505 & 11.25 & 0.000 & .4919 & .6994622 \\
\hline
\end{tabular}


Modelol - Completo Monday December 21 11:32:26 2015 Page 2

4 .

end of do-file

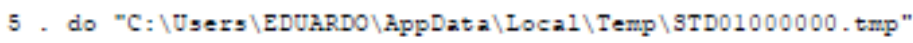

6 . estat ic

\begin{tabular}{c|cccccc}
\hline Kodel & Obs & 11 (nul1) & 11 (model) & df & AIC & BIC \\
\hline & 65 & & -60.22376 & 6 & 136.4475 & 153.8426 \\
\hline
\end{tabular}

7 . estat vee, corr

Correlation matrix of coefficients of arima model

\begin{tabular}{|c|c|c|c|c|c|c|c|c|}
\hline$e(\mathrm{~V})$ & $\begin{array}{r}\text { infanotu } \\
\text { leitos }\end{array}$ & tmin & $27.4 \mathrm{rm} \sim \mathrm{n}$ & 25. comax & interv 0 & L3. int $\sim 0$ & $\begin{array}{l}\text { ARMR } \\
\quad \text { I14.25 }\end{array}$ & $\begin{array}{l}\text { signa } \\
\text { cons }\end{array}$ \\
\hline $\begin{array}{r}\text { infartotx } \\
\text { leitos } \\
\text { tmin } \\
27 \text {.urmmin } \\
\text { L5. comax } \\
\text { intervencao } \\
\text { L3.interve o }\end{array}$ & $\begin{array}{r}1.0000 \\
-0.4645 \\
-0.8742 \\
0.3174 \\
-0.2353 \\
0.2330\end{array}$ & $\begin{array}{r}1.0000 \\
0.0901 \\
-0.7244 \\
0.1412 \\
-0.1304\end{array}$ & $\begin{array}{r}1.0000 \\
-0.2600 \\
0.1244 \\
-0.1997\end{array}$ & $\begin{array}{r}1.0000 \\
-0.0023 \\
0.0941\end{array}$ & $\begin{array}{r}1.0000 \\
-0.8718\end{array}$ & 1.0000 & & \\
\hline I14.ar & -0.0981 & 0.0324 & 0.1034 & -0.0289 & -0.0201 & -0.0116 & 1.0000 & \\
\hline $\begin{array}{l}\text { zigma } \quad \text { cons } \\
\text { nand }\end{array}$ & -0.0159 & 0.0052 & 0.0167 & -0.0046 & -0.0035 & -0.0016 & 0.1627 & 1.0000 \\
\hline
\end{tabular}

8 - predict resido, I

( 7 missing values generated)

9. xcorr infartotu resido, table

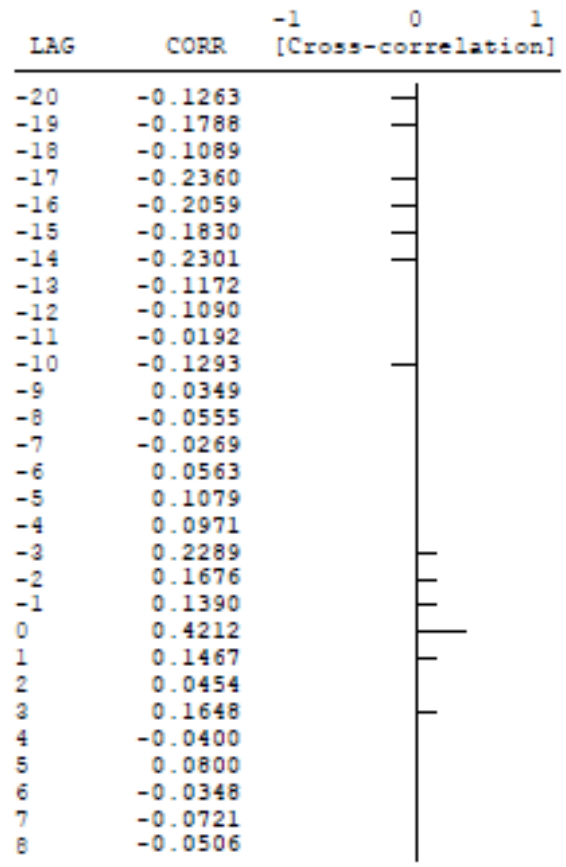




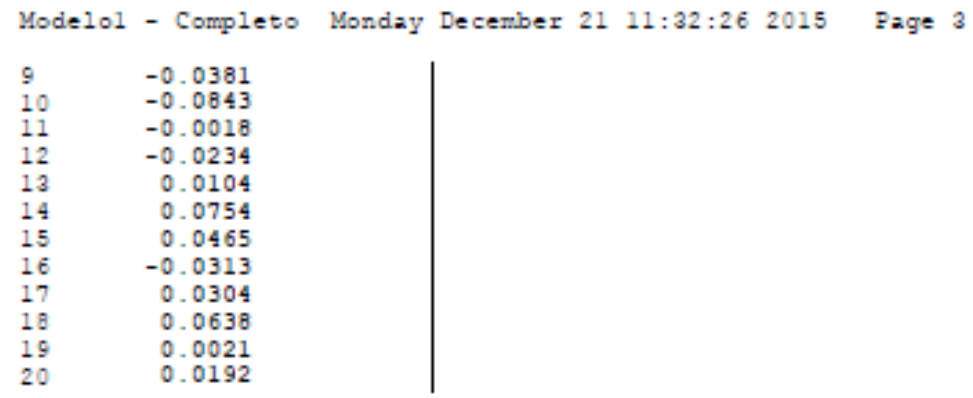

10. corrgram resido

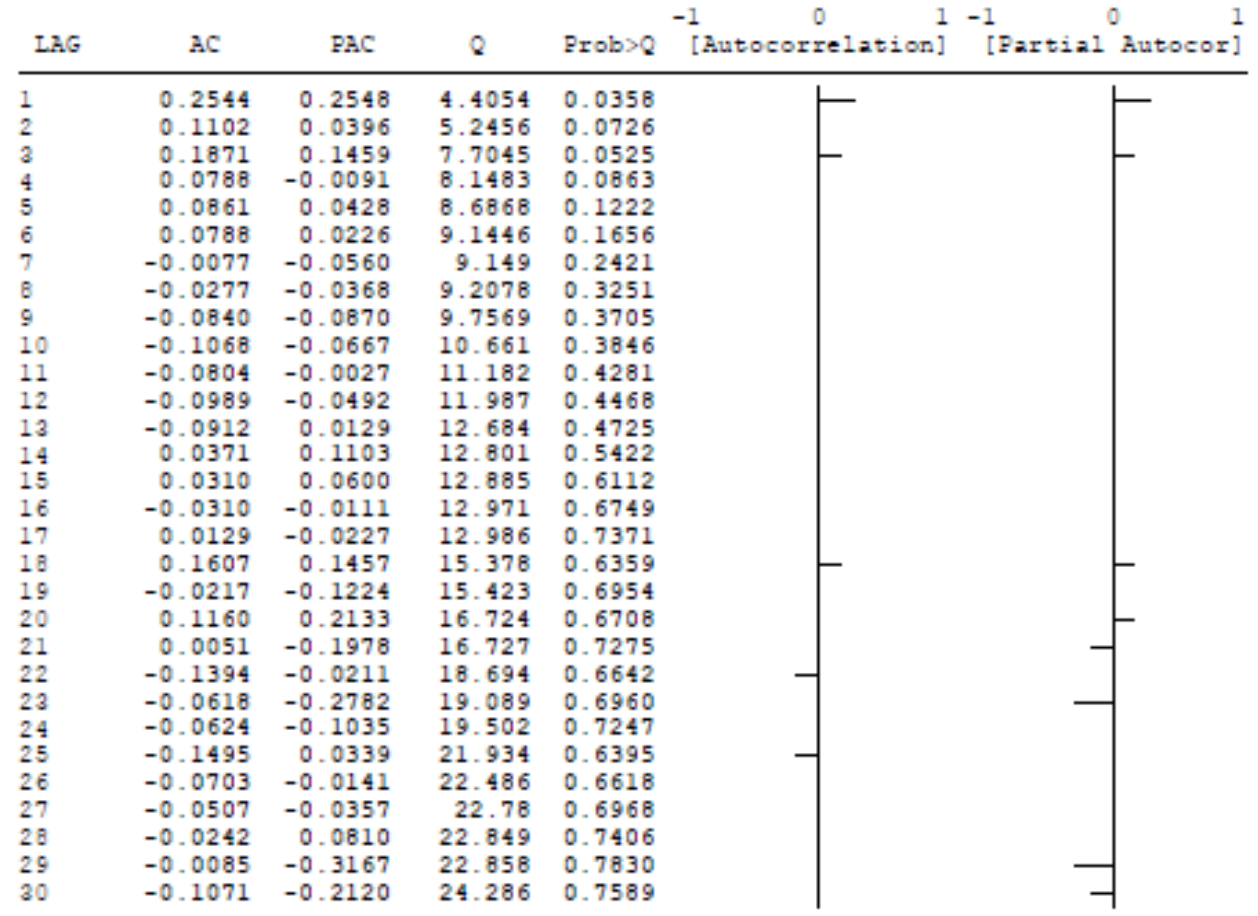

11 . pnorm resido

12 . swilk resido

Shapiro-Wilk w test for normal data

\begin{tabular}{r|rcccc} 
Variable & Obs & W & $V$ & $z$ & Prob>z \\
\hline resido & 65 & 0.98354 & 0.954 & -0.102 & 0.54071
\end{tabular}

13 . sum resido

\begin{tabular}{r|rrrrr} 
Variable & Obs & Mean & Std. Dev & Min & Max \\
\hline resido & 65 & .0218666 & .6232921 & -1.37495 & 1.352804
\end{tabular}


Anexos

Anexo B: Modelo estimado para taxa de internações por "AVC", metodologia ARIMAX

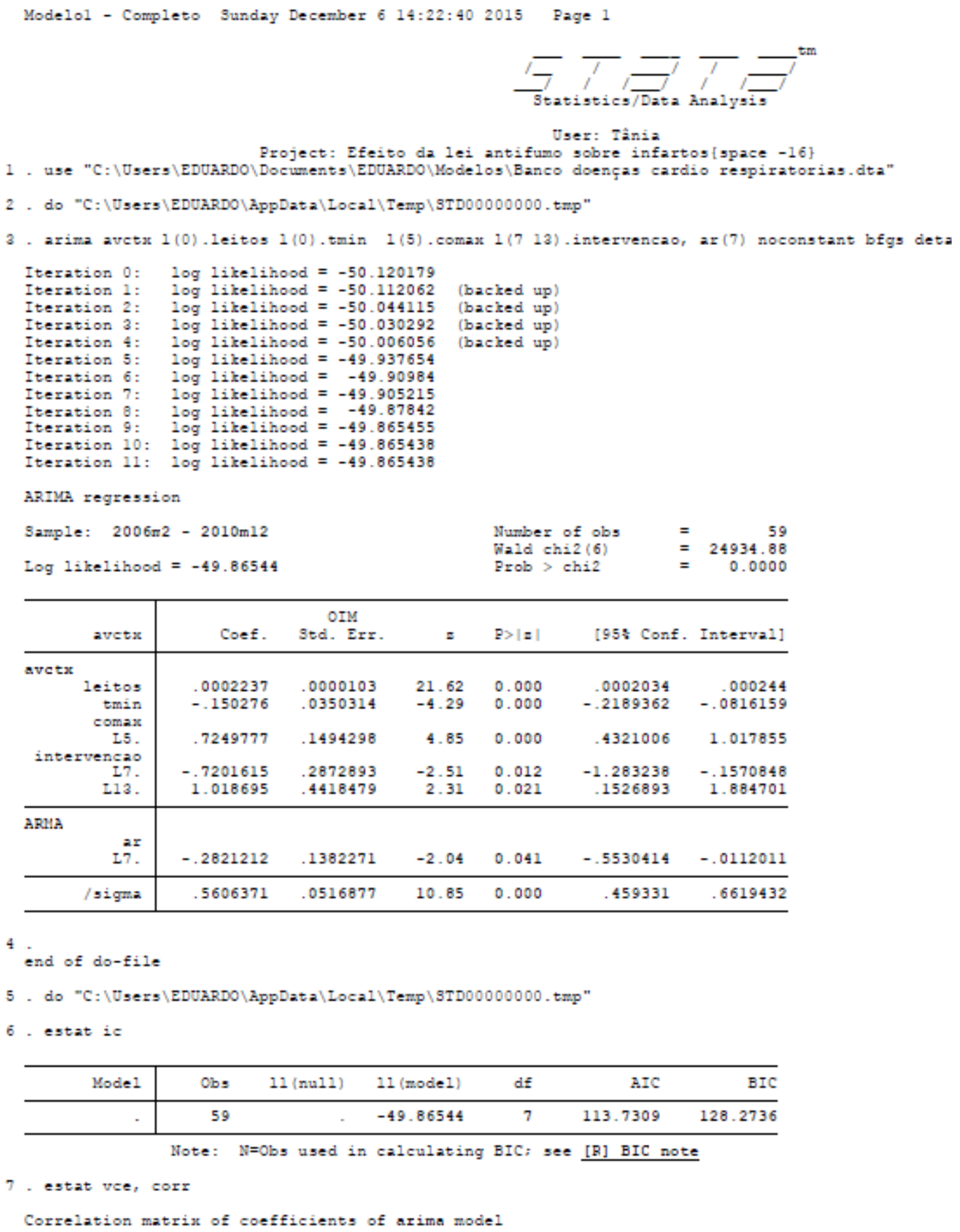

\begin{tabular}{|c|c|c|c|c|c|c|}
\hline avets & Coef. & $\begin{array}{c}\text { OIM } \\
\text { Std. Err. }\end{array}$ & $z$ & P> $|z|$ & [95s Conf. & Interval] \\
\hline \multicolumn{7}{|l|}{$\operatorname{svctx}$} \\
\hline leitos & .0002237 & .0000103 & 21.62 & 0.000 & .0002034 & .000244 \\
\hline $\operatorname{tmin}$ & -.150276 & .0350314 & -4.29 & 0.000 & -.2189362 & -.0816159 \\
\hline I5. & .7249777 & .1494298 & 4.85 & 0.000 & .4321006 & 1.017855 \\
\hline intervencao & & & & & & \\
\hline $\begin{array}{r}\text { I7. } \\
\text { L13. }\end{array}$ & $\begin{array}{r}-.7201615 \\
1.018695\end{array}$ & $\begin{array}{l}.2872893 \\
.4418479\end{array}$ & $\begin{array}{r}-2.51 \\
2.31\end{array}$ & $\begin{array}{l}0.012 \\
0.021\end{array}$ & $\begin{array}{r}-1.283238 \\
.1526893\end{array}$ & $\begin{array}{r}-.1570848 \\
1.884701\end{array}$ \\
\hline \multicolumn{7}{|l|}{ ARMA } \\
\hline L7. & -.2821212 & .1382271 & -2.04 & 0.041 & -.5530414 & -.0112011 \\
\hline /sigma & .5606371 & .0516877 & 10.85 & 0.000 & .459331 & .6619432 \\
\hline
\end{tabular}

end of do-file

5 . do "C: $\backslash$ Users $\backslash E D U A R D O \backslash$ AppData $\backslash$ Local $\backslash$ Temp $\backslash$ STD00000000 . tmp"

6. estat ic

\begin{tabular}{r|rrrrrr}
\hline Nodel & Obs & 11 (nu11) & 11 (mode1) & de & AIC & BIC \\
\hline & 59 & & -49.86544 & 7 & 113.7309 & 128.2736 \\
\hline \multicolumn{5}{c}{ Note: N=Obs used in calculating } & BIC; see [R] BIC note \\
\cline { 1 - 1 }
\end{tabular}

7. estat vee, corr

Correlation matrix of coefficients of arima model

4 


\begin{tabular}{|c|c|c|c|c|c|c|c|}
\hline$e(V)$ & $\begin{array}{l}\text { avetu } \\
\text { leitos }\end{array}$ & tmin & 25. $\cos 2 x$ & 27. int $\sim 0$ & I. .int $\sim 0$ & $\begin{array}{l}\text { ARMA } \\
27.2 x\end{array}$ & $\begin{array}{l}\text { sigma } \\
\text { cons }\end{array}$ \\
\hline $\begin{array}{r}\text { avctur leitos } \\
\text { tmin } \\
\text { L5. comax } \\
\text { 27.interve } 0 \\
\text { L13. interv } 0\end{array}$ & $\begin{array}{r}1.0000 \\
-0.8391 \\
0.0754 \\
-0.5336 \\
0.3710\end{array}$ & $\begin{array}{r}1.0000 \\
-0.5942 \\
0.4072 \\
-0.3555\end{array}$ & $\begin{array}{r}1.0000 \\
-0.0171 \\
0.1141\end{array}$ & $\begin{array}{r}1.0000 \\
-0.7327\end{array}$ & 1.0000 & & \\
\hline 27.2x & -0.1820 & 0.2257 & -0.1615 & 0.2529 & -0.3231 & 1.0000 & \\
\hline 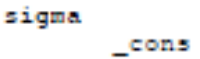 & -0.0099 & 0.0123 & -0.0088 & 0.0138 & -0.0176 & 0.0545 & 1.0000 \\
\hline
\end{tabular}

B - predict resido, r

(13 missing values generated)

9. xcorr avetu resido, table

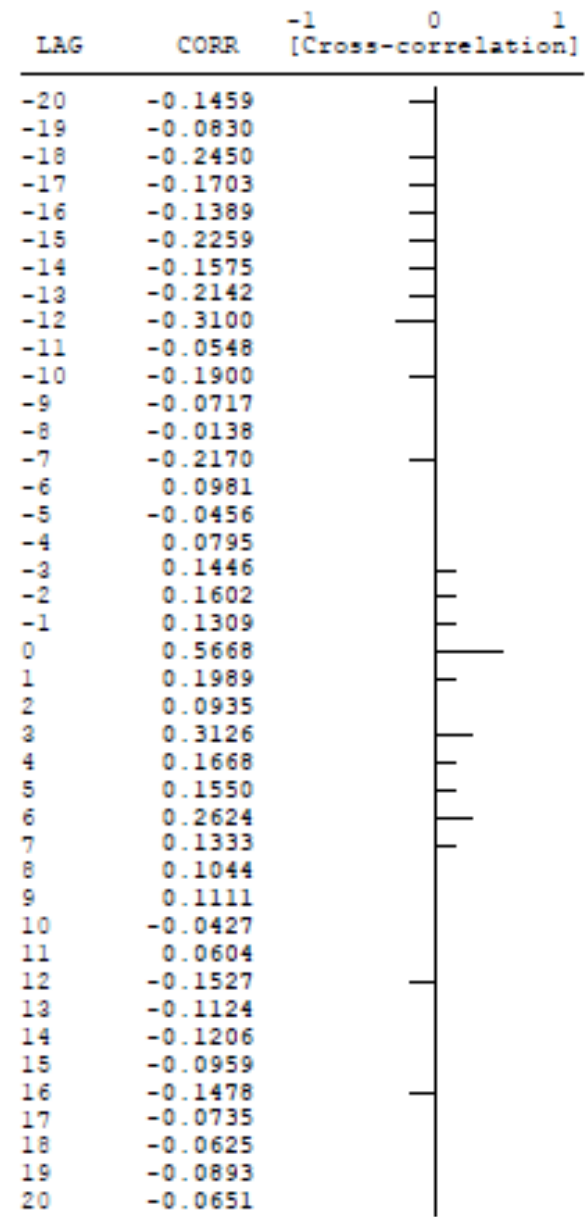


Modelol - Completo Sunday December 6 14:22:41 2015 Page 3

10. corrgram resido

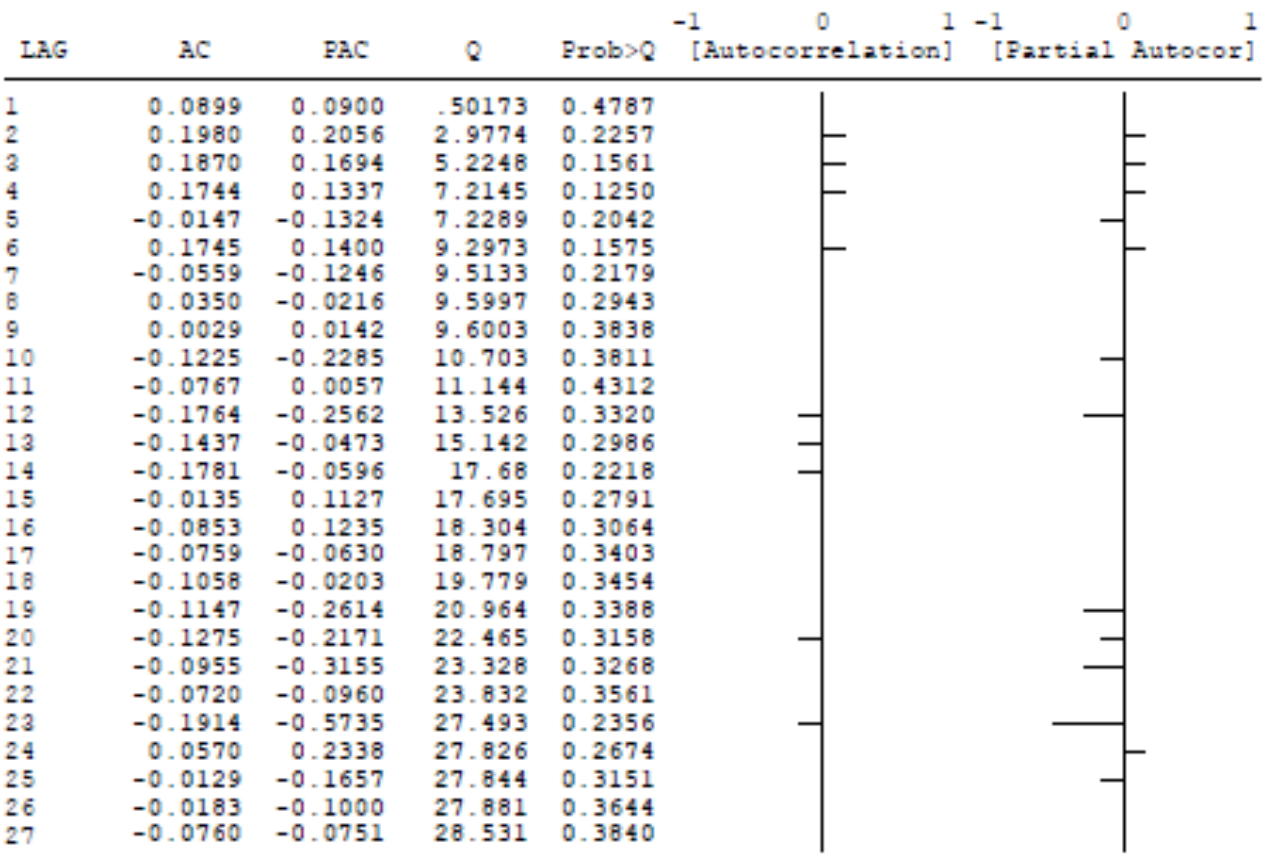

11 . pnorm resido

swilk resido

Shapiro-Wilk w test for normal data

\begin{tabular}{r|rcccr} 
Variable & Obs & W & $V$ & $z$ & Prob>z \\
\hline resido & 59 & 0.98921 & 0.579 & -1.177 & 0.88040
\end{tabular}

13 . sum resido

\begin{tabular}{r|rrrrr} 
Variable & Obs & Mean & Std. Dev & Min & Max \\
\hline resido & 59 & -.0059257 & .567011 & -1.20304 & 1.525969
\end{tabular}

14

end of do-file 
Anexos

Anexo C: Modelo estimado para taxa de mortalidade por "Infarto", metodologia ARIMAX

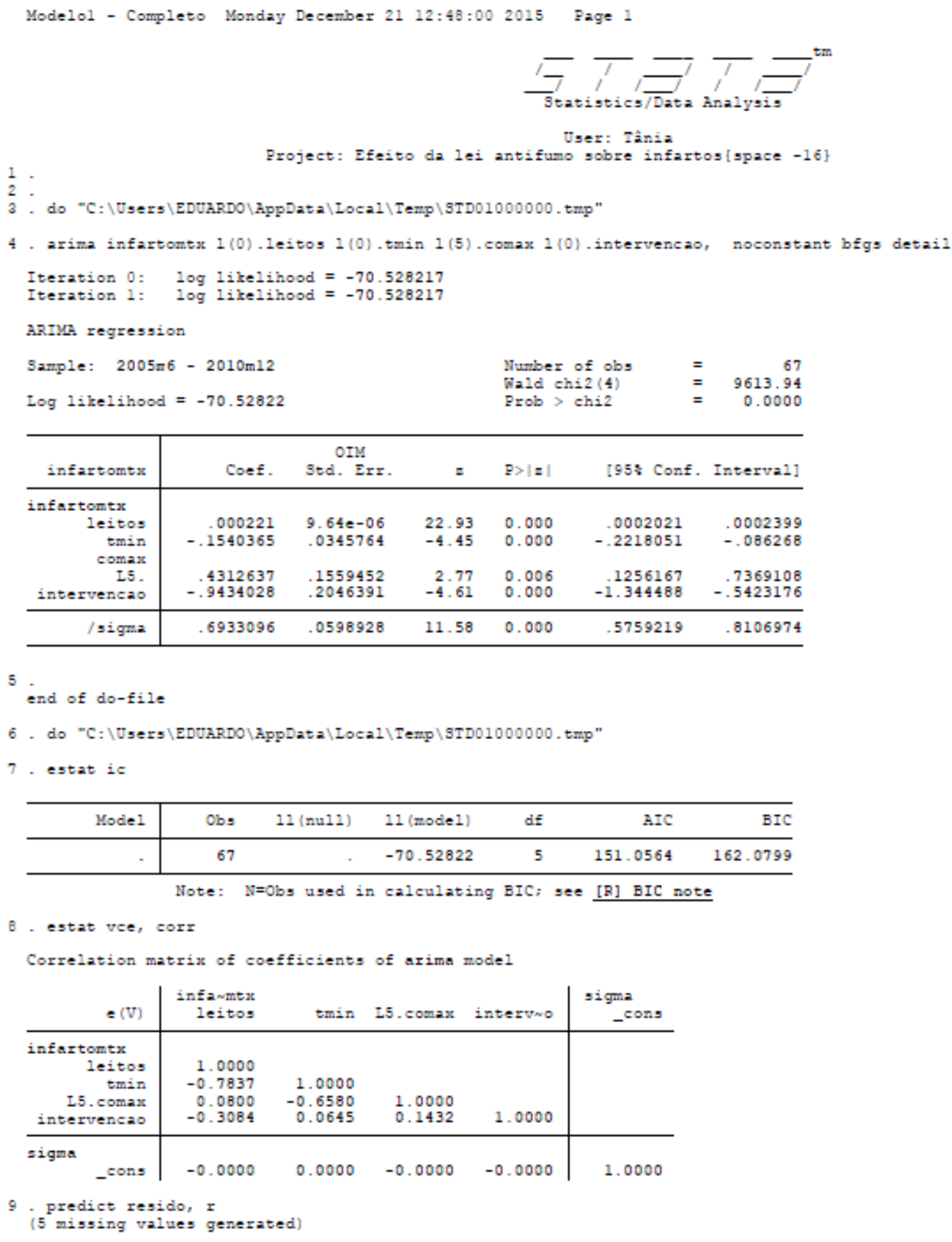

\begin{tabular}{|c|c|c|c|c|c|c|}
\hline infartomta & Coef. & $\begin{array}{c}\text { OIM } \\
\text { Std. Err. }\end{array}$ & $z$ & $P>\mid=1$ & [95: Conf. & Interval] \\
\hline \multicolumn{7}{|l|}{ infartontu } \\
\hline leitos & .000221 & $9.64 e-06$ & 22.93 & 0.000 & .0002021 & .0002399 \\
\hline tmin & -.1540365 & .0345764 & -4.45 & 0.000 & -.2218051 & -.086268 \\
\hline L5. & .4312637 & .1559452 & 2.77 & 0.006 & .1256167 & .7369108 \\
\hline intervencao & -.9434028 & .2046391 & -4.61 & 0.000 & -1.344488 & -.5423176 \\
\hline /sigma & .6933096 & .0598928 & 11.58 & 0.000 & .5759219 & .8106974 \\
\hline
\end{tabular}

5 end of do-file

\begin{tabular}{|c|c|c|c|c|c|c|}
\hline Mode1 & $\mathrm{Obs}$ & 11 (nu11) & 11 (mode1) & df & AIC & BIC \\
\hline . & 67 & & -70.52822 & 5 & 151.0564 & 162.0799 \\
\hline
\end{tabular}

8. estat vee, corr

Correlation matrix of coefficient, of arima model

\begin{tabular}{|c|c|c|c|c|c|}
\hline$=(\mathrm{V})$ & $\begin{array}{r}\text { infawmex } \\
\text { leitos }\end{array}$ & $\operatorname{tmin}$ & L5. $\cos 2 x$ & interv 0 & $\begin{array}{l}\text { sigma } \\
\text { cons }\end{array}$ \\
\hline $\begin{array}{r}\text { infartomtx } \\
\text { leitos } \\
\text { tmin } \\
\text { L5. comax } \\
\text { intervencao }\end{array}$ & $\begin{array}{r}1.0000 \\
-0.7837 \\
0.0800 \\
-0.3084\end{array}$ & $\begin{array}{r}1.0000 \\
-0.6580 \\
0.0645\end{array}$ & $\begin{array}{l}1.0000 \\
0.1432\end{array}$ & 1.0000 & \\
\hline cons & -0.0000 & 0.0000 & -0.0000 & -0.0000 & 1.0000 \\
\hline
\end{tabular}

9 . predict resido, $r$

(5 missing values generated) 


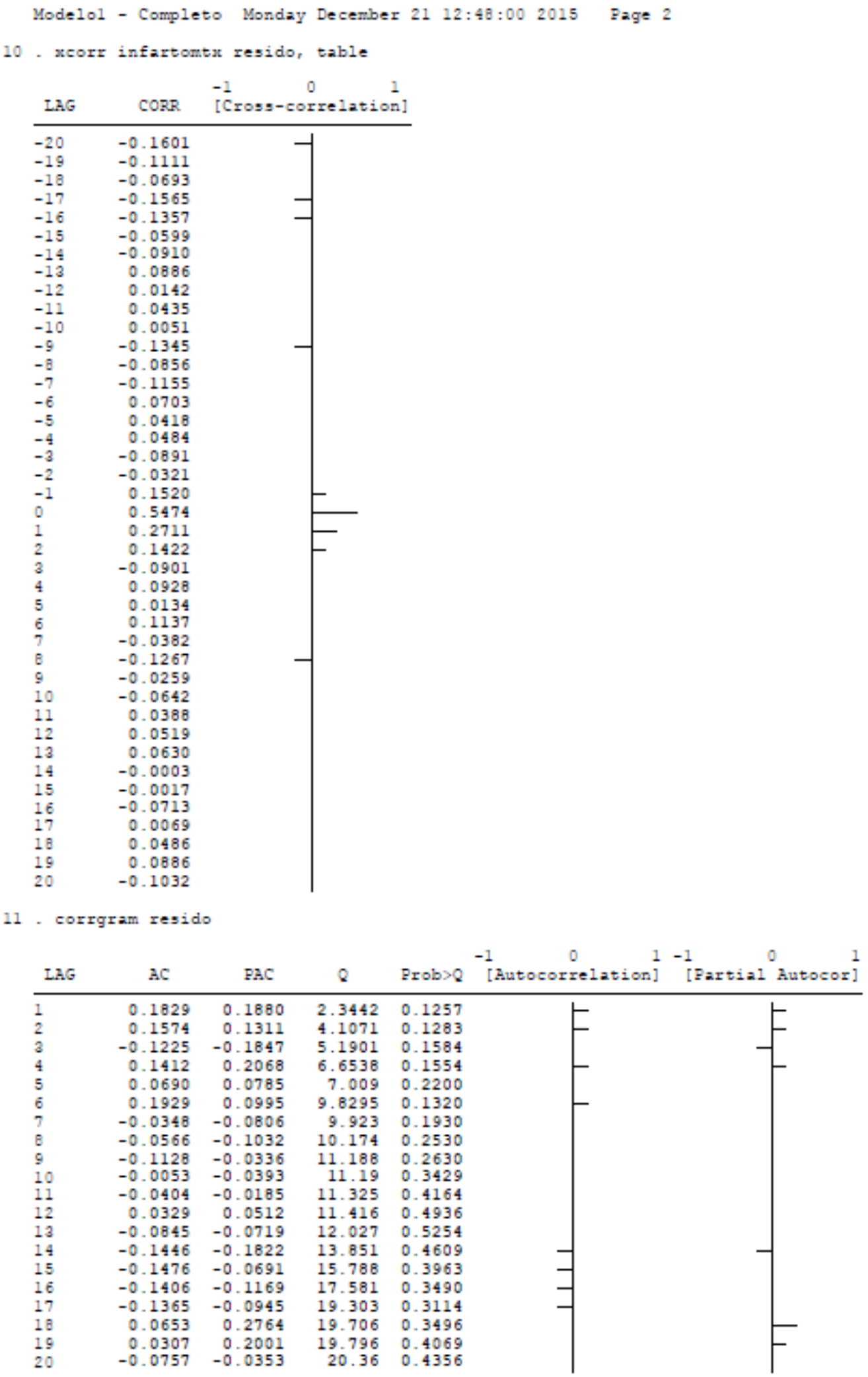




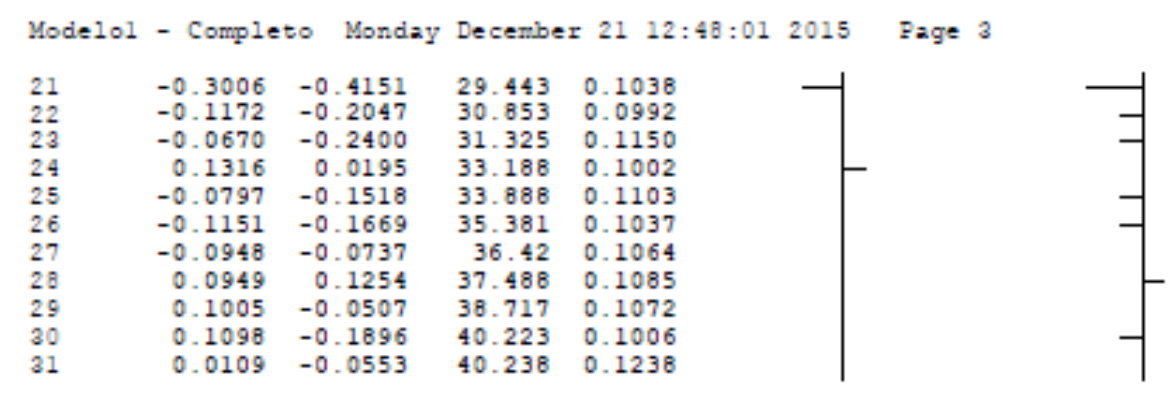

12 . pnorm resido

13. swilk resido

Shapiro-Wilk $W$ test for normal data

\begin{tabular}{r|ccccc} 
Variable & Obs & W & V & $z$ & Prob>z \\
\hline resido & 67 & 0.97383 & 1.555 & 0.957 & 0.16927
\end{tabular}

14. sum resido

\begin{tabular}{r|rrrrr} 
Variable & Obs & Mean & Std. Dev & Min & Max \\
\hline resido & 67 & .016888 & .698335 & -1.387731 & 1.944575
\end{tabular}

15.

end of do-file

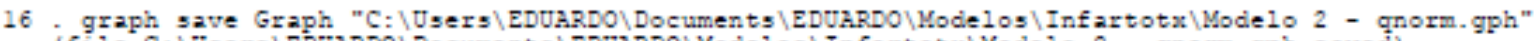

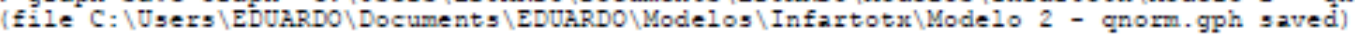

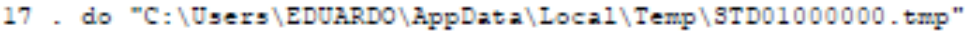

18. ac resido 


\section{Anexo D: Modelo estimado para taxa de mortalidade por "AVC", metodologia} ARIMAX

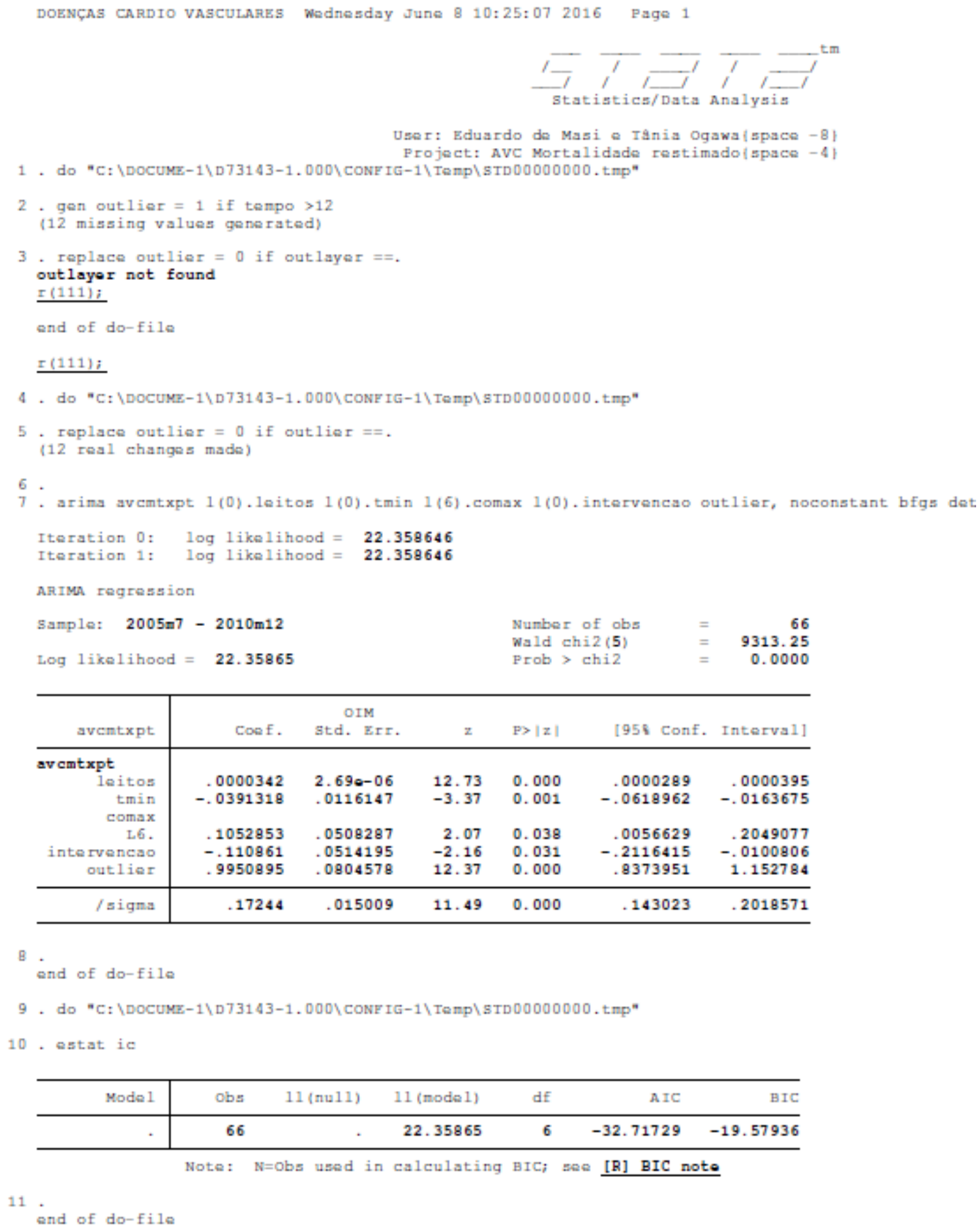

\begin{tabular}{|c|c|c|c|c|c|c|}
\hline avcentxpt & $\cos f$. & $\begin{array}{c}\text { OrM } \\
\text { std. Err. }\end{array}$ & $z$ & P> $|z|$ & [958 Conf. & Interval] \\
\hline \multicolumn{7}{|l|}{ avcmtxpt } \\
\hline $\begin{array}{r}\text { leitos } \\
\text { tmin }\end{array}$ & $\begin{array}{r}.0000342 \\
-.0391318\end{array}$ & $\begin{array}{l}2.690-06 \\
.0116147\end{array}$ & $\begin{array}{l}12.73 \\
-3.37\end{array}$ & $\begin{array}{l}0.000 \\
0.001\end{array}$ & $\begin{array}{r}.0000289 \\
-.0618962\end{array}$ & $\begin{array}{r}.0000395 \\
-.0163675\end{array}$ \\
\hline $\begin{array}{r}\text { L6. } \\
\text { intervencao } \\
\text { outiler }\end{array}$ & $\begin{array}{r}1052853 \\
-.110861 \\
.9950895\end{array}$ & $\begin{array}{r}.0508287 \\
.0514195 \\
.0804578\end{array}$ & $\begin{array}{r}2.07 \\
-2.16 \\
12.37\end{array}$ & $\begin{array}{l}0.038 \\
0.031 \\
0.000\end{array}$ & $\begin{array}{r}.0056629 \\
-.2116415 \\
.8373951\end{array}$ & $\begin{array}{r}.2049077 \\
-.0100806 \\
1.152784\end{array}$ \\
\hline /signa & .17244 & .015009 & 11.49 & 0.000 & .143023 & 2018571 \\
\hline
\end{tabular}
end of do-file

\begin{tabular}{r|rrrrrr}
\hline Model & Obs & 11 (nul1) & 11 (mode 1 ) & df & AIC & BIC \\
\hline$\cdot$ & 66 & & 22.35865 & 6 & $\mathbf{- 3 2 . 7 1 7 2 9}$ & $\mathbf{- 1 9 . 5 7 9 3 6}$ \\
\hline
\end{tabular}

Note: N=Obs used in calculating BIC; see [R] BIC note end of do-file

11. 
DOENÇAs CARDIO VAsculares Wednesday June 8 10:25:07 2016 Page 2

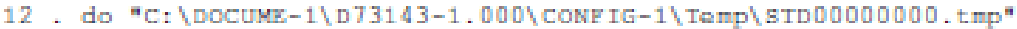

13. estat vee, corr

Correlation matrix of coefficients of arima model

\begin{tabular}{|c|c|c|c|c|c|c|}
\hline$\simeq(V)$ & $\begin{array}{l}\text { aventxpt } \\
\text { leitos }\end{array}$ & $\operatorname{tmin}$ & L6. comax & interv-o & outlier & $\begin{array}{l}\text { sigms } \\
\text { cons }\end{array}$ \\
\hline $\begin{array}{r}\text { avcmtxpt } \\
\text { leitos } \\
\text { tmin } \\
\text { L6. comax } \\
\text { intervencao } \\
\text { outlier }\end{array}$ & $\begin{array}{r}1.0000 \\
-0.6142 \\
0.2057 \\
-0.2086 \\
-0.3215\end{array}$ & $\begin{array}{r}1.0000 \\
-0.8237 \\
0.0313 \\
-0.3424\end{array}$ & $\begin{array}{l}1.0000 \\
0.1224 \\
0.2999\end{array}$ & $\begin{array}{r}1.0000 \\
-0.0928\end{array}$ & 1.0000 & \\
\hline -cons & -0.0000 & 0.0000 & -0.0000 & -0.0000 & -0.0000 & 1.0000 \\
\hline
\end{tabular}

14.

and of do-file

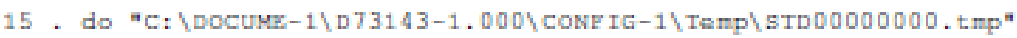

16 . predict resido, r

( 6 missing values generated)

17.

end of do-file

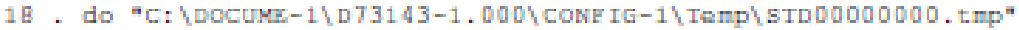

19. xcorr avcntxpt resido, table

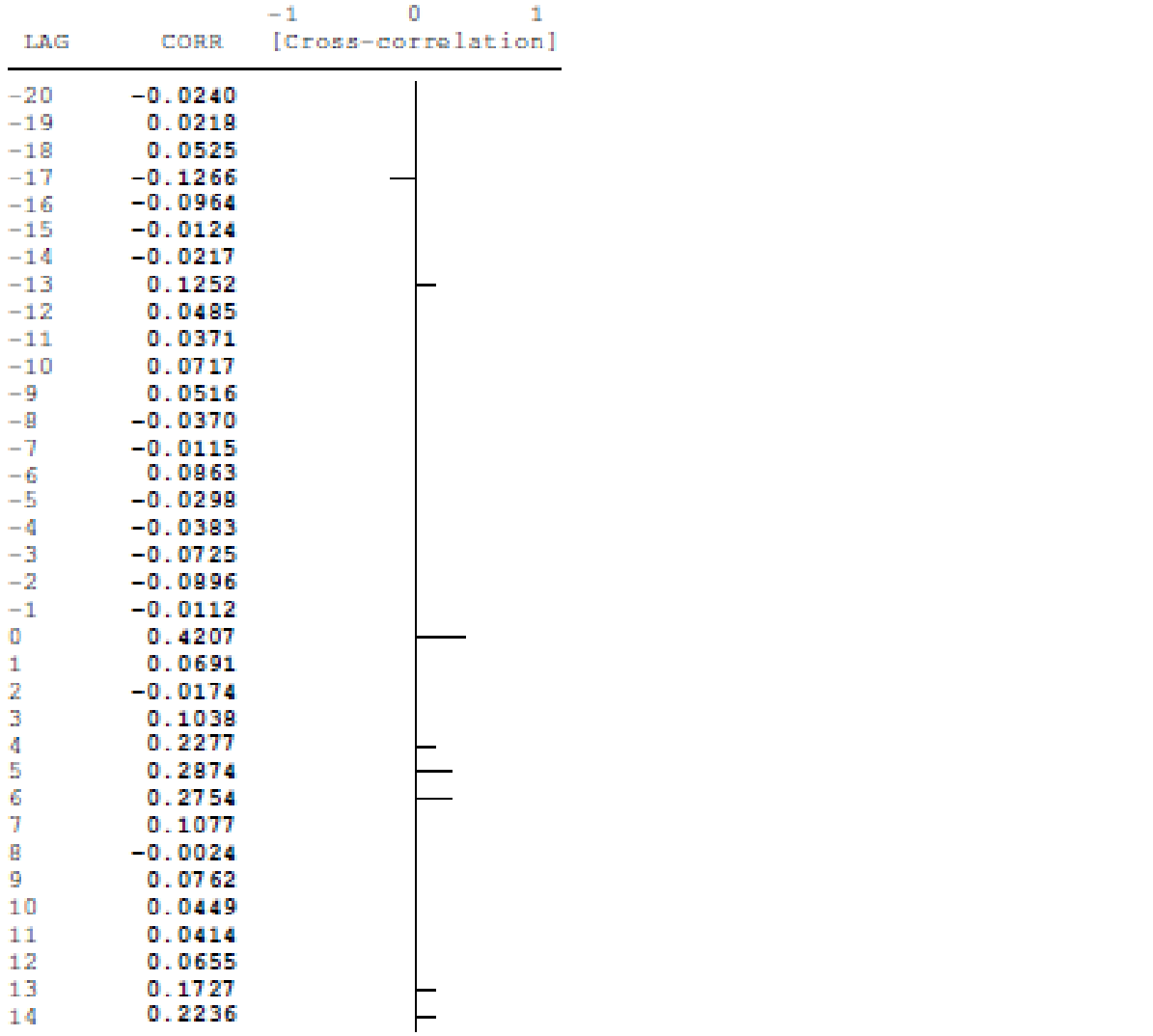


DOENÇA CARDIO VAsCuLARES Wednesday June 8 10:25:07 2016 Page 3

$\begin{array}{llll}1.5 & 0.2285 & \\ 16 & 0.1826 & \\ 17 & 0.2539 & \\ 18 & 0.3340 & \\ 19 & 0.3024 & \\ 20 & 0.0823 & \end{array}$

20. corrgram resido

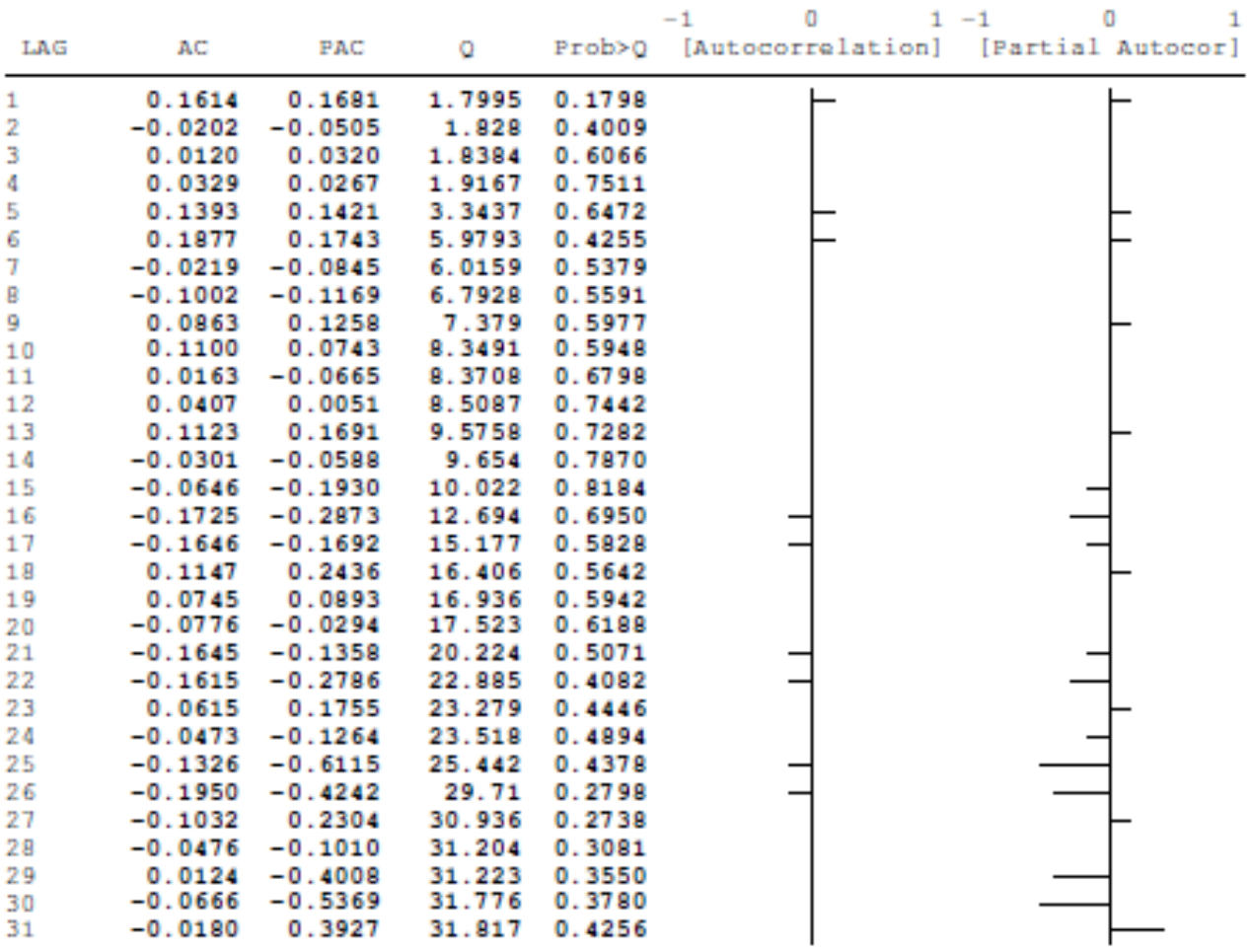

21.

and of do-file

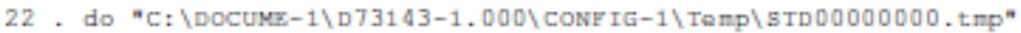

23. swilk resido

Shapiro-wilk w test for normal data

\begin{tabular}{c|ccccc} 
Variable & obs & w & V & z & Prob $>z$ \\
\hline resido & 66 & 0.97616 & 1.399 & 0.728 & 0.23331
\end{tabular}

24 . sum resido

\begin{tabular}{c|ccccc} 
Variable & obs & Moan & Std. Dev & Min & Max \\
\hline resido & 66 & .0027709 & .173739 & -.4554271 & .318488
\end{tabular}

25

and of do-file

26. 


\section{Anexo E: Modelo estimado para taxa de internações por "Infarto", metodologia} ITSA-ARIMAX

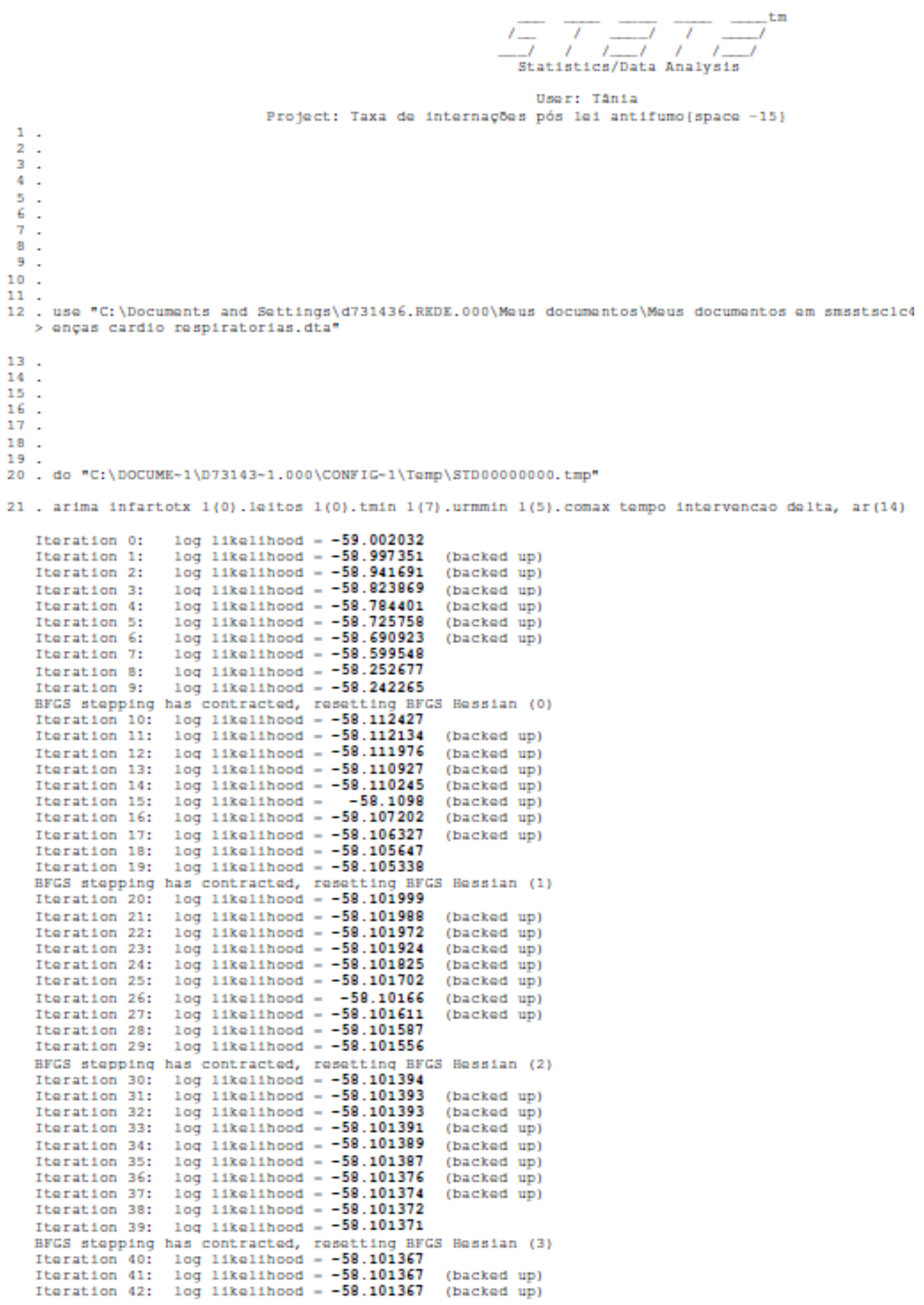




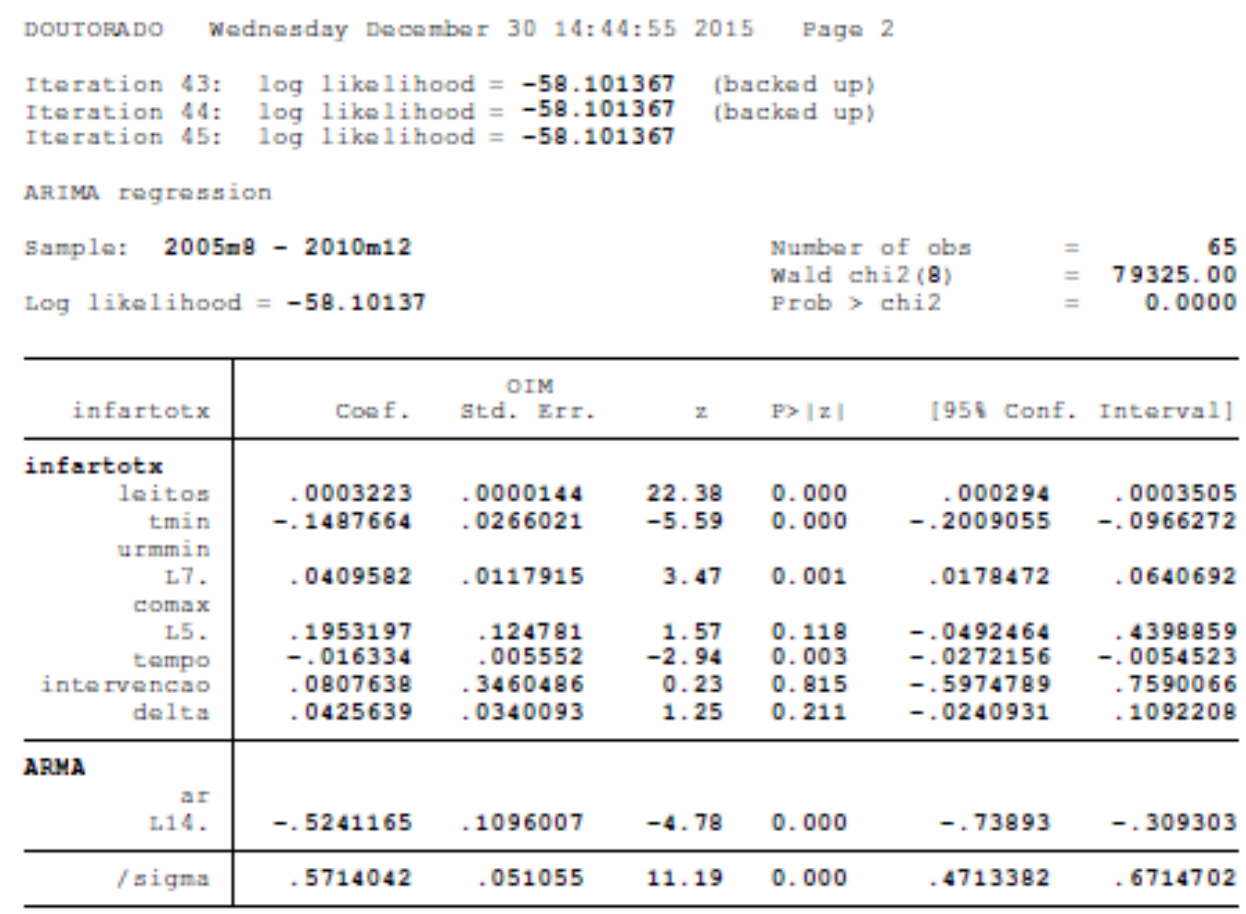

22 .

end of do-file

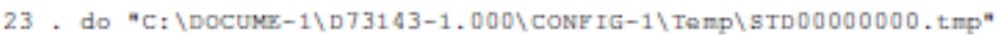

24 . estat. ic

\begin{tabular}{|c|c|c|c|c|c|c|}
\hline Mode 1 & $\mathrm{obs}$ & 11 (nu11) & 11 (node 1 ) & df & AIC & BIC \\
\hline . & 65 & . & -58.10137 & 9 & 134.2027 & 153.7722 \\
\hline
\end{tabular}

25. estat vee, corr

Correlation matrix of coefficients of arima nodel

\begin{tabular}{|c|c|c|c|c|c|c|c|c|}
\hline$\simeq(V)$ & $\begin{array}{r}\text { infa-otx } \\
\text { leitos }\end{array}$ & tmin & L7. $4 \mathrm{rm}-\mathrm{n}$ & 2.5. coms $x$ & tempo & interv-o & delta & $\begin{array}{l}\text { ARMA } \\
\text { L14.ar }\end{array}$ \\
\hline $\begin{array}{r}\text { infartotx } \\
\text { leitos } \\
\text { tmin } \\
\text { 47.urmmin } \\
\text { L5. comax } \\
\text { tempo } \\
\text { intervencao } \\
\text { delta }\end{array}$ & $\begin{array}{r}1.0000 \\
-0.3450 \\
-0.8511 \\
0.2025 \\
-0.3230 \\
0.1021 \\
0.0612\end{array}$ & $\begin{array}{r}1.0000 \\
0.0398 \\
-0.7386 \\
-0.2048 \\
0.0594 \\
0.1082\end{array}$ & $\begin{array}{r}1.0000 \\
-0.2152 \\
0.0939 \\
-0.0276 \\
-0.0877\end{array}$ & $\begin{array}{r}1.0000 \\
0.2558 \\
-0.0400 \\
-0.0439\end{array}$ & $\begin{array}{r}1.0000 \\
-0.4698 \\
-0.1227\end{array}$ & $\begin{array}{r}1.0000 \\
-0.6913\end{array}$ & 1.0000 & \\
\hline ARMA & -0.1520 & -0.0060 & 0.1198 & 0.0213 & 0.1595 & -0.1671 & 0.0779 & 1. 0000 \\
\hline _cons & -0.0290 & -0.0012 & 0.0229 & 0.0041 & 0.0305 & -0.0319 & 0.0149 & 0.1910 \\
\hline
\end{tabular}


Anexos

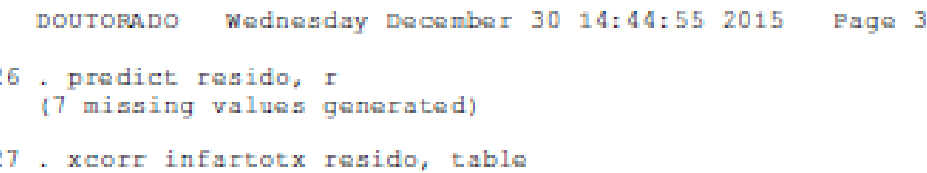

\begin{tabular}{|c|c|c|}
\hline LAG & CORR & $\begin{array}{ccc}-1 & 0 & 1 \\
\text { [Crosz-correlation] }\end{array}$ \\
\hline-20 & 0.0287 & \\
\hline-19 & -0.0346 & \\
\hline-18 & 0.0293 & \\
\hline-17 & -0.1226 & \\
\hline-16 & -0.1028 & \\
\hline-15 & -0.1125 & \\
\hline-14 & -0.1823 & - \\
\hline-13 & -0.0808 & \\
\hline-12 & -0.1002 & \\
\hline-11 & 0.0154 & \\
\hline-10 & -0.1013 & \\
\hline-9 & 0.0756 & \\
\hline-8 & 0.0060 & \\
\hline-7 & 0.0195 & \\
\hline-6 & 0.1492 & - \\
\hline-5 & 0.1500 & - \\
\hline-4 & 0.1462 & - \\
\hline-3 & 0.2289 & - \\
\hline-2 & 0.1633 & - \\
\hline-1 & 0.1176 & \\
\hline 0 & 0.3767 & \begin{tabular}{|l} 
\\
\end{tabular} \\
\hline 1 & 0.1060 & \\
\hline 2 & -0.0346 & \\
\hline 3 & 0.1117 & \\
\hline 4 & -0.1232 & \\
\hline 5 & 0.0036 & \\
\hline 6 & -0.0826 & \\
\hline 7 & -0.1499 & - \\
\hline$B$ & -0.0736 & \\
\hline 9 & -0.0908 & \\
\hline 10 & -0.1500 & 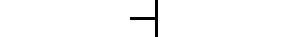 \\
\hline 11 & -0.0854 & \\
\hline 12 & -0.1173 & \\
\hline 13 & -0.0720 & \\
\hline 14 & 0.0062 & \\
\hline 15 & -0.0345 & \\
\hline 16 & -0.1012 & \\
\hline 17 & -0.0060 & \\
\hline 18 & 0.0610 & \\
\hline 19 & 0.0160 & \\
\hline 20 & 0.0723 & \\
\hline
\end{tabular}

28 . corrgram resido

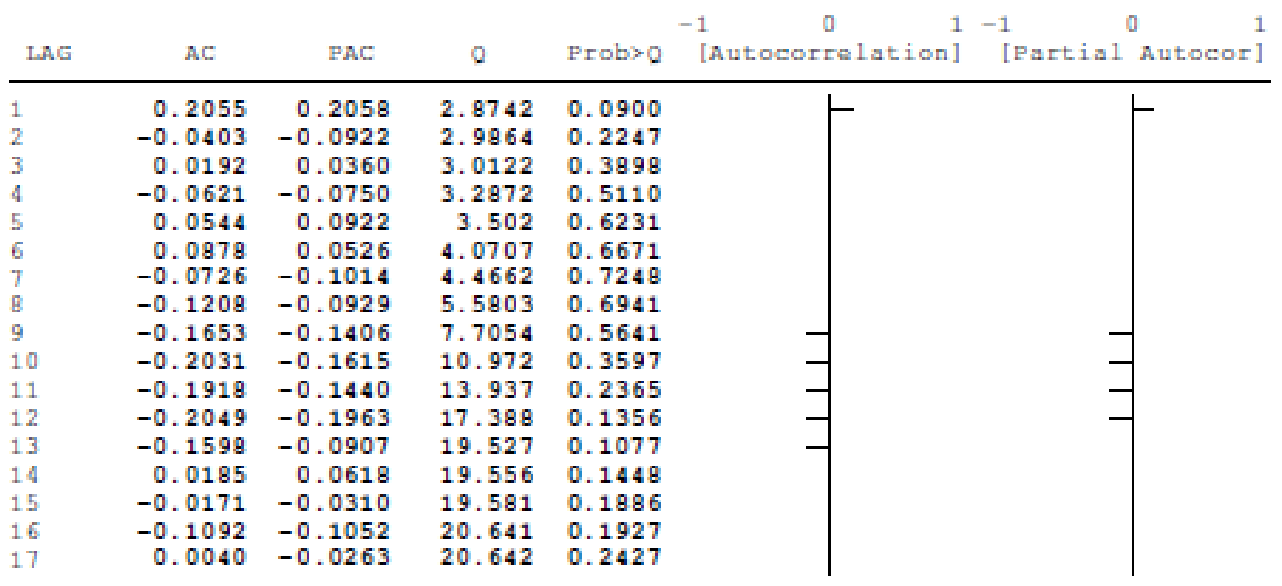




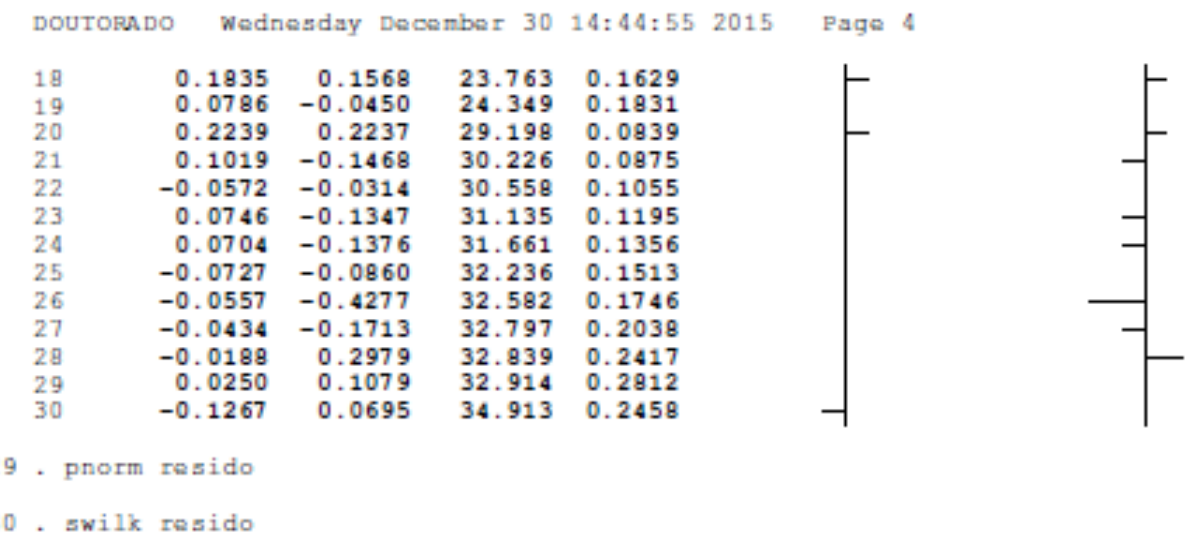

Shapiro-wilk w test for normal data

\begin{tabular}{r|rrrrr} 
Variable & obs & W & V & z & Prob>z \\
\hline resido & 65 & 0.97597 & 1.393 & 0.718 & 0.23639
\end{tabular}

31. sum resido

\begin{tabular}{r|rrrrr} 
Variable & obs & Mean & Std. Dev. & Min & Max \\
\hline resido & 65 & -.0015876 & .6056203 & -1.643786 & 1.124761
\end{tabular}

32 . end of do-file

33 . graph save Graph "C:\Documents and Settings \d731436. REDE.000\Meus documentos\Meus documentos $>$ elos\ARIMA_ITSA infartotx \pnorm_nodelo1.gph", replace

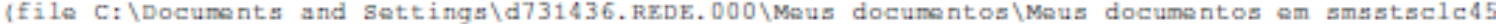
$>$ A \infartotx (pnorn_modelo1.gph saved)

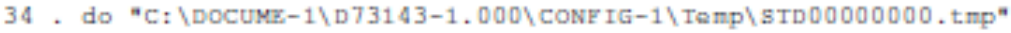

35 . ac resido

36. end of do-file

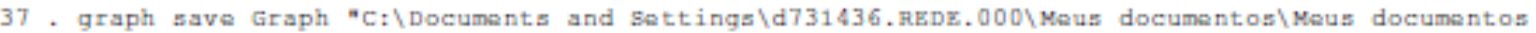

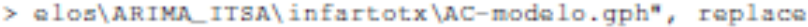

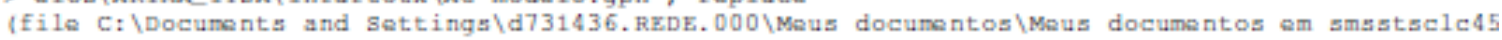
$>A \backslash$ infartotx $\backslash A C-m o d e l o . g p h$ saved)

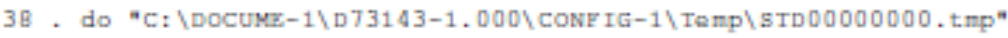

39 . pac resido

40. end of do-file

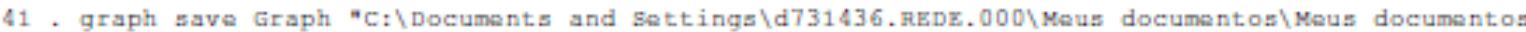
$>$ elos\ARIMA_ITSA infartotx\PAC-modelo.gph", replace

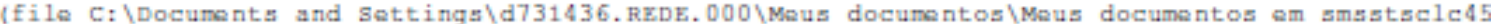
$>$ A \infartotx $\backslash P A C-n o d e l o . g p h ~ s a v e d\}$ 


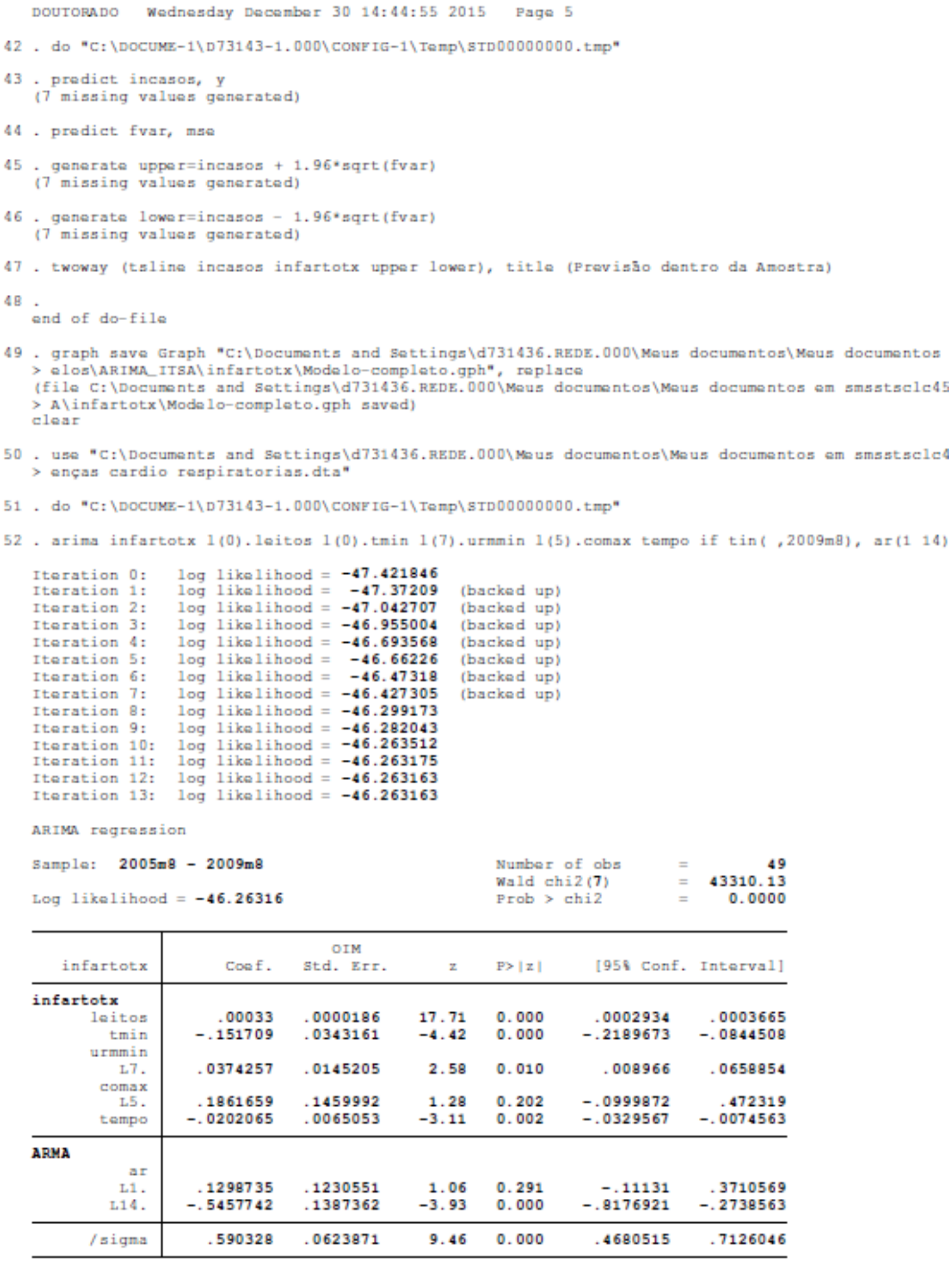

\begin{tabular}{|c|c|c|c|c|c|c|}
\hline infartot $x$ & $\operatorname{Cos} \mathrm{f}$. & $\begin{array}{c}\text { OIM } \\
\text { std. Err. }\end{array}$ & $z$ & P> $|z|$ & [95\& Conf. & Intervall \\
\hline \multicolumn{7}{|l|}{ infartot $x$} \\
\hline $\begin{array}{r}\text { leitos } \\
\text { tmin } \\
\text { urmmin }\end{array}$ & $\begin{array}{r}.00033 \\
-.151709\end{array}$ & $\begin{array}{l}.0000186 \\
.0343161\end{array}$ & $\begin{array}{l}17.71 \\
-4.42\end{array}$ & $\begin{array}{l}0.000 \\
0.000\end{array}$ & $\begin{array}{r}.0002934 \\
-.2189673\end{array}$ & $\begin{array}{r}.0003665 \\
-.0844508\end{array}$ \\
\hline L7. & .0374257 & .0145205 & 2.58 & 0.010 & .008966 & .0658854 \\
\hline $\begin{array}{r}\text { comax } \\
\text { L5. } \\
\text { tempo }\end{array}$ & $\begin{array}{r}.1861659 \\
-.0202065\end{array}$ & $\begin{array}{l}.1459992 \\
.0065053\end{array}$ & $\begin{array}{r}1.28 \\
-3.11\end{array}$ & $\begin{array}{l}0.202 \\
0.002\end{array}$ & $\begin{array}{l}-.0999872 \\
-.0329567\end{array}$ & $\begin{array}{r}.472319 \\
-.0074563\end{array}$ \\
\hline \multicolumn{7}{|l|}{ ARMA } \\
\hline ar & & & & & & \\
\hline $\begin{array}{l}\text { L1. } \\
\text { L14. }\end{array}$ & $\begin{array}{r}.1298735 \\
-.5457742\end{array}$ & $\begin{array}{l}.1230551 \\
.1387362\end{array}$ & $\begin{array}{r}1.06 \\
-3.93\end{array}$ & $\begin{array}{l}0.291 \\
0.000\end{array}$ & $\begin{array}{r}-.11131 \\
-.8176921\end{array}$ & $\begin{array}{r}.3710569 \\
-.2738563\end{array}$ \\
\hline / signa & .590328 & .0623871 & 9.46 & 0.000 & .4680515 & .7126046 \\
\hline
\end{tabular}


Dourokado Wednesday Decenber $30 \quad 14: 44: 552015$ Page 6

53

end of do-file

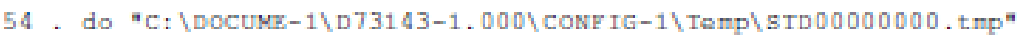

55. estat ic

\begin{tabular}{r|rrrrrr}
\hline Node 1 & obs & 11 (nul1) & 11 (node 1$)$ & df & AIC & BIC \\
\hline$\cdot$ & 49 & & -46.26316 & 8 & 108.5263 & 123.6609 \\
\hline
\end{tabular}

Note: N=Obs used in calculating BIC; see [R] BIC note

56. estat vee, corr

Correlation matrix of coefficients of arima nodel

\begin{tabular}{|c|c|c|c|c|c|c|c|c|}
\hline$\simeq(V)$ & $\begin{array}{r}\text { infa-otx } \\
\text { leitos }\end{array}$ & $\operatorname{tmin}$ & L7. urm-n & L.5. cons $x$ & tempo & $\begin{array}{l}\text { AFMA } \\
\text { L.ar }\end{array}$ & L14.ar & $\begin{array}{l}\text { sigms } \\
\quad \text { cons }\end{array}$ \\
\hline $\begin{array}{r}\text { infartotx } \\
\text { leitos } \\
\text { tmin } \\
\text { 27.urmmin } \\
\text { L5. comax } \\
\text { tempo }\end{array}$ & $\begin{array}{r}1.0000 \\
-0.3973 \\
-0.8469 \\
0.1936 \\
-0.3389\end{array}$ & $\begin{array}{r}1.0000 \\
0.0518 \\
-0.7303 \\
-0.1702\end{array}$ & $\begin{array}{r}1.0000 \\
-0.1729 \\
0.1250\end{array}$ & $\begin{array}{l}1.0000 \\
0.2681\end{array}$ & 1.0000 & & & \\
\hline $\begin{array}{r}\text { L. ar } \\
\text { L14.ar }\end{array}$ & $\begin{array}{l}-0.2834 \\
-0.2968\end{array}$ & $\begin{array}{l}0.0522 \\
0.0499\end{array}$ & $\begin{array}{l}0.3150 \\
0.2553\end{array}$ & $\begin{array}{l}-0.0487 \\
-0.0115\end{array}$ & $\begin{array}{r}-0.0094 \\
0.2211\end{array}$ & $\begin{array}{l}1.0000 \\
0.4112\end{array}$ & 1.0000 & \\
\hline _cons & -0.0815 & 0.0135 & 0.0677 & -0.0019 & 0.0679 & 0.0929 & 0.2923 & 1.0000 \\
\hline
\end{tabular}

57 . predict resido, I

(7 misaing values generated)

5B. xcorr infartotx resido, table

\begin{tabular}{|c|c|c|}
\hline LAG & CORR & $\begin{array}{lcc}-1 & 0 & 1 \\
\text { [Cross-correlation] }\end{array}$ \\
\hline-20 & 0.0331 & \\
\hline-19 & -0.0083 & \\
\hline-18 & 0.0654 & \\
\hline-17 & -0.1000 & \\
\hline-16 & -0.0548 & \\
\hline-15 & -0.0766 & \\
\hline-14 & -0.1432 & -1 \\
\hline-13 & -0.0381 & \\
\hline-12 & -0.0868 & \\
\hline-11 & 0.0468 & \\
\hline-10 & -0.0712 & \\
\hline-9 & 0.1288 & - \\
\hline-8 & 0.0650 & \\
\hline-7 & 0.0984 & \\
\hline-6 & 0.2418 & - \\
\hline-5 & 0.2232 & - \\
\hline-4 & 0.2254 & - \\
\hline-3 & 0.2997 & 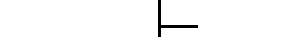 \\
\hline-2 & 0.2188 & - \\
\hline-1 & 0.1953 & - \\
\hline 0 & 0.4279 & \begin{tabular}{|l} 
\\
\end{tabular} \\
\hline 1 & 0.1660 & - \\
\hline 2 & 0.0735 & \\
\hline 3 & 0.2449 & - \\
\hline 4 & 0.0163 & \\
\hline 5 & 0.1419 & - \\
\hline 6 & 0.0354 & \\
\hline 7 & -0.0435 & \\
\hline
\end{tabular}




$$
\begin{array}{r}
-0.0102 \\
-0.0511 \\
-0.1231 \\
-0.0640 \\
-0.1123 \\
-0.0450 \\
0.0187 \\
-0.0012 \\
-0.0719 \\
0.0099 \\
0.0550 \\
-0.0430 \\
-0.0121
\end{array}
$$

59. corrgram resido

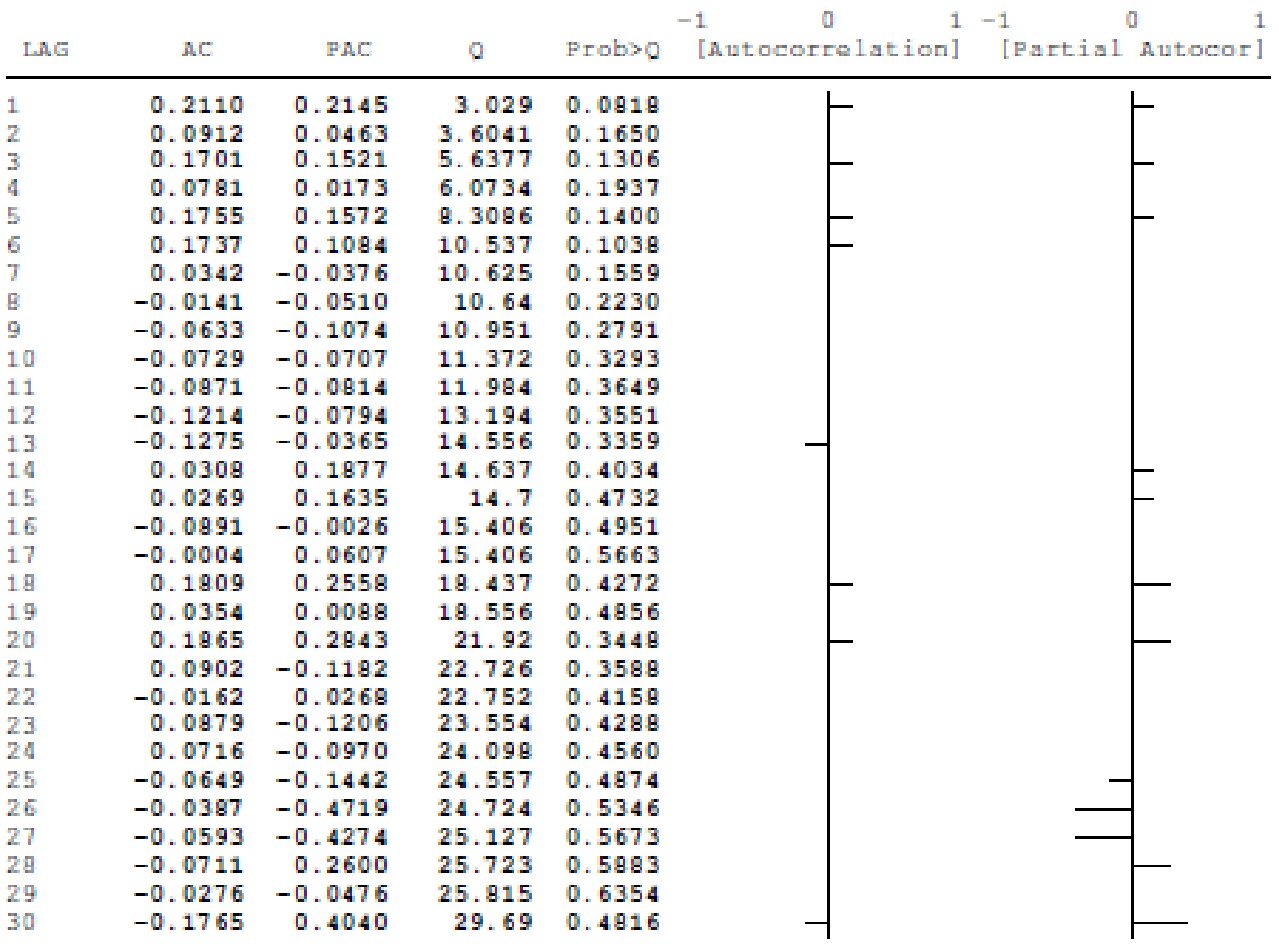

60 . pnorm resido

61. swilk resido

shapiro-wilk w test for normal data

\begin{tabular}{c|ccccc} 
Variable & obs & w & V & z & Prob>z \\
\hline resido & 65 & 0.97088 & 1.688 & 1.134 & 0.12839
\end{tabular}

62 . sum resido

\begin{tabular}{r|rrrrr} 
Variable & obs & Mean & Std. Dev & Min & Max \\
\hline resido & 65 & .1497094 & .6552381 & -1.525236 & 1.290184
\end{tabular}

63.

end of do-file

64. 
Anexos

Anexo F: Modelo estimado para taxa de internações por "AVC", metodologia ITSAARIMAX

DOUTOHADO Wednsaday Desambar 30 15:44:02 2015 Page

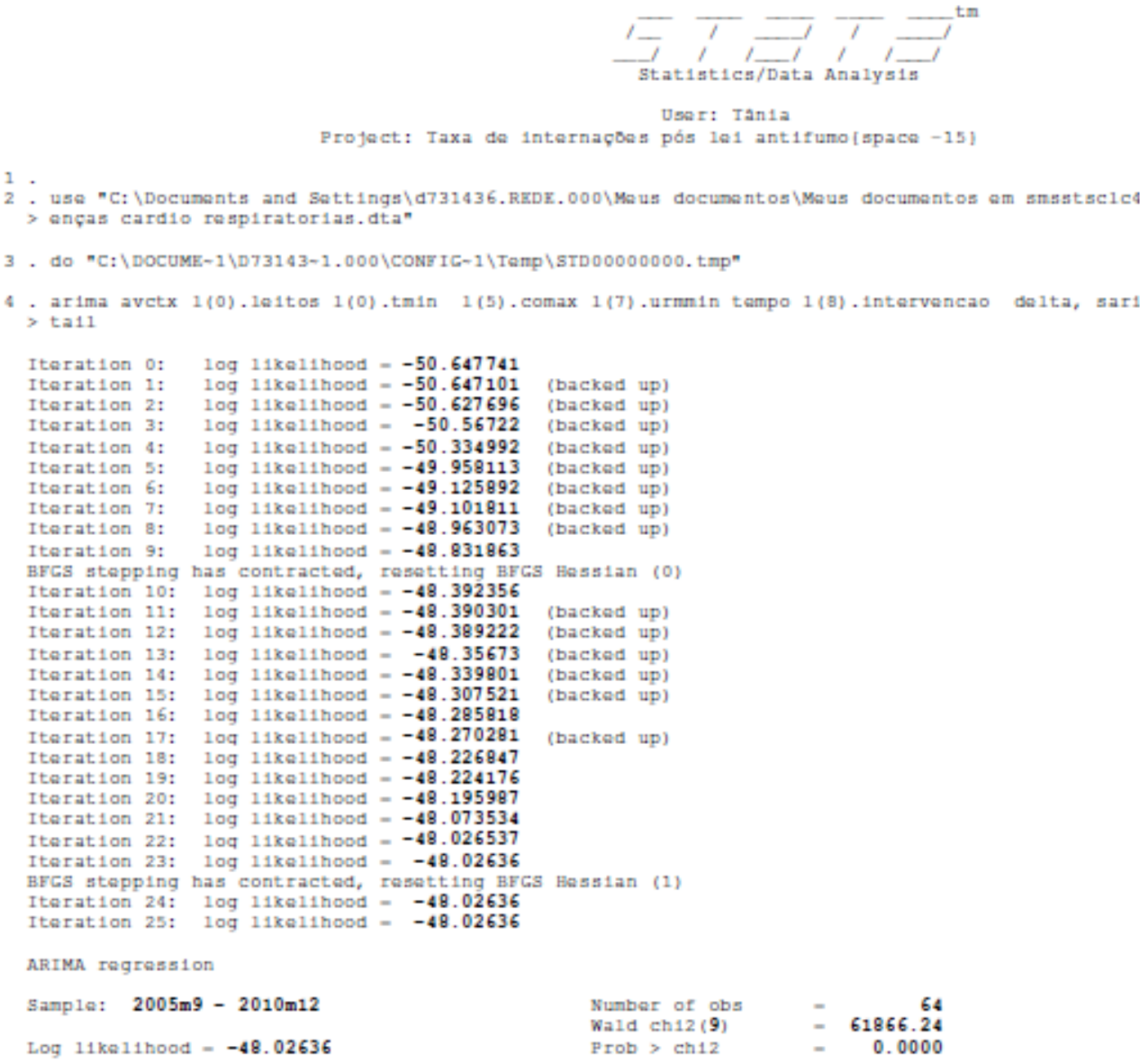

Nugber of obs Wald ch12(9) Prob $>$ ch 12

64
$-\quad 61866.24$

0.0000

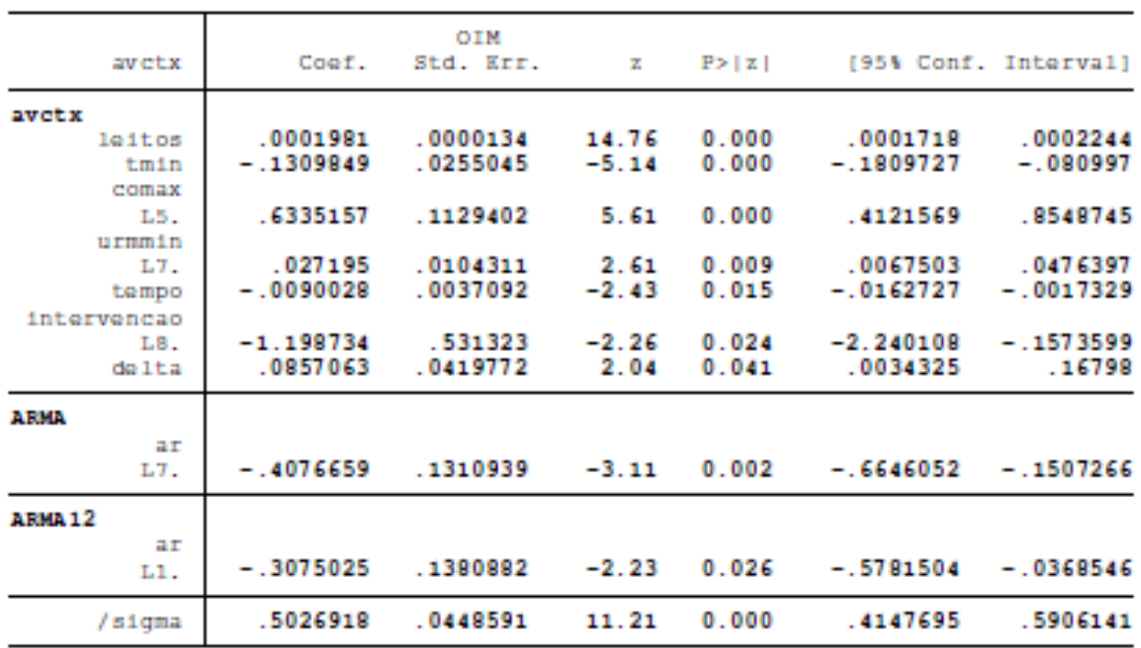




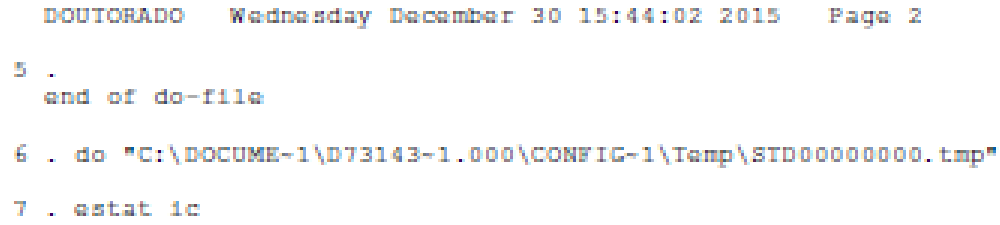

\begin{tabular}{|c|c|c|c|c|c|c|}
\hline Moden 1 & Obs & 11 (nul1) & 11 (modn 1$)$ & de & AIC & BIC \\
\hline . & 64 & . & -48.02636 & 10 & 116.0527 & 137.6416 \\
\hline
\end{tabular}

8. astat ves, corr

Correlation matrix of confficiants of arima modal

\begin{tabular}{|c|c|c|c|c|c|c|c|c|}
\hline$\approx(V)$ & $\begin{array}{l}\text { avct. } x \\
\quad \ln 1 \mathrm{t} t o s\end{array}$ & $\operatorname{tm} 1 \mathrm{n}$ & L5. comax & LT. uran-n & tempo & LB. $1 \mathrm{nt}-0$ & delta & $\begin{array}{l}\text { A PRMA } \\
\text { L.7. aI }\end{array}$ \\
\hline $\begin{array}{r}\text { avetx } \\
\text { lestos } \\
\text { tmin } \\
\text { L5. comax } \\
\text { L7. urmmin } \\
\text { tempo } \\
\text { L8.1ntervaro } \\
\text { den } 1 \text { ta }\end{array}$ & $\begin{array}{r}1.0000 \\
-0.5631 \\
0.2752 \\
-0.7686 \\
-0.3394 \\
-0.0940 \\
0.1857\end{array}$ & $\begin{array}{r}1.0000 \\
-0.5535 \\
0.1105 \\
0.0112 \\
0.2694 \\
-0.1997\end{array}$ & $\begin{array}{r}1.0000 \\
-0.3973 \\
0.2047 \\
-0.0812 \\
0.1003\end{array}$ & $\begin{array}{r}1.0000 \\
0.0469 \\
-0.1052 \\
-0.0162\end{array}$ & $\begin{array}{r}1.0000 \\
0.1779 \\
-0.4341\end{array}$ & $\begin{array}{r}1.0000 \\
-0.9018\end{array}$ & 1. 0000 & \\
\hline ARMA & 0.0763 & -0.0587 & 0.0596 & -0.0900 & 0.0641 & 0.1625 & -0.1258 & 1.0000 \\
\hline ARMA 12 & -0.0194 & 0.0747 & -0.0740 & -0.0458 & 0.1318 & 0.0497 & -0.0519 & 0.1981 \\
\hline _cons & 0.0041 & 0.0027 & -0.0026 & -0.0116 & 0.0180 & 0.0177 & -0.0150 & 0.0981 \\
\hline
\end{tabular}

9 . pradict rasido, r

(8 missing valuss gensatad)

$10 . x \operatorname{cor} r$ avetx rasido, tabla

\begin{tabular}{|c|c|c|}
\hline & & $\begin{array}{lll}-1 & 0 & 1\end{array}$ \\
\hline ILAG & CORR & [Cross-correlation] \\
\hline-20 & 0.0125 & \\
\hline-19 & 0.0248 & \\
\hline-18 & -0.1668 & - \\
\hline-17 & -0.0573 & \\
\hline-16 & -0.1295 & - \\
\hline-15 & -0.1314 & - \\
\hline-14 & -0.0805 & \\
\hline-13 & -0.1620 & - \\
\hline-12 & -0.2314 & - \\
\hline-11 & -0.0502 & \\
\hline-10 & -0.1913 & - \\
\hline-9 & -0.0879 & \\
\hline-8 & -0.0428 & \\
\hline-7 & -0.3108 & $\rightarrow$ \\
\hline-6 & -0.0522 & \\
\hline-5 & -0.1073 & \\
\hline-4 & -0.0272 & \\
\hline-3 & 0.0004 & \\
\hline-2 & -0.0486 & \\
\hline-1 & -0.0203 & \\
\hline 0 & 0.4631 & \\
\hline 1 & 0.1162 & \\
\hline 2 & 0.0611 & \\
\hline 3 & 0.2582 & - \\
\hline 4 & 0.0468 & \\
\hline
\end{tabular}


DOUTORADO Wednasday Desambar 30 15:44:02 2015 Pago 3

\begin{tabular}{lr|l}
5 & -0.0603 & \\
6 & 0.1287 & \\
7 & 0.0559 & \\
8 & -0.0062 & \\
9 & 0.0088 & \\
10 & -0.0427 & \\
11 & 0.0791 & \\
12 & 0.0045 & \\
13 & -0.0330 & \\
14 & -0.0548 & \\
15 & 0.0205 & \\
16 & -0.0390 & \\
17 & -0.0040 & \\
18 & 0.0221 & \\
19 & -0.0859 & \\
20 & -0.0718 &
\end{tabular}

11 . corrgram rasido

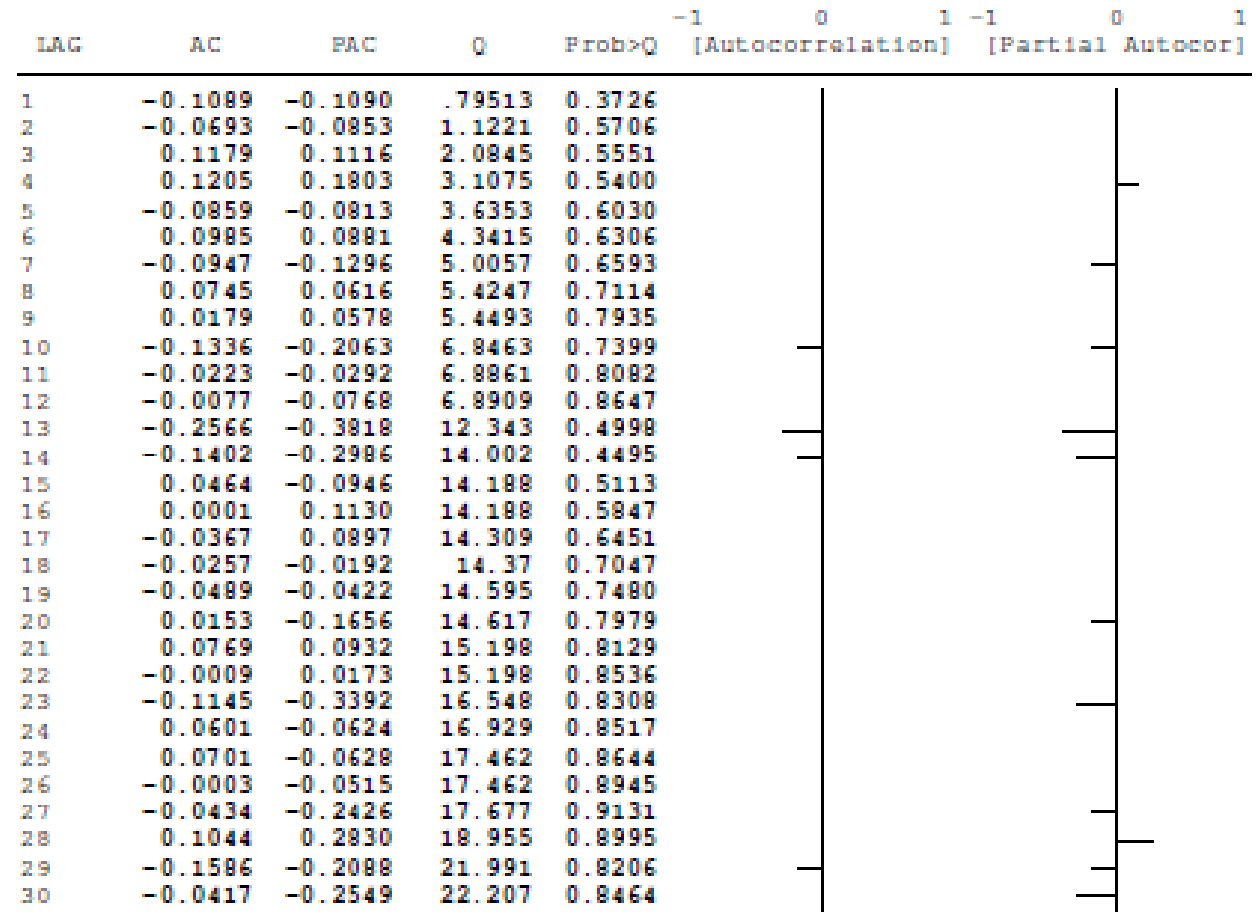

12 . pnorm resido

13 . 3 w1 $11 \mathrm{k}$ rasido

Shapiro-W11k $\mathrm{W}$ tast for normal data

\begin{tabular}{c|ccccc} 
Var1abia & Obs & w & V & z & Prob>z \\
\hline rasido & 64 & 0.98496 & 0.861 & -0.324 & 0.62718
\end{tabular}


DOUTORADO Wednesday Decomber 30 15:44:02 2015 Page 4

14 . sum resido

\begin{tabular}{r|rrrrrr} 
Var1abla & Obs & Man & Std. Dev & Nin & Max \\
\hline resido & 64 & -.0157569 & .5122421 & -1.064961 & 1.192225
\end{tabular}

15. and of do-tile

16 . do "C: $\backslash$ DOCUME-1 $\backslash D 73143-1.000 \backslash C O N E I G-1 \backslash$ T $2 m p \backslash S T D 00000000 . t m p "$

17 . ac rasido

18. snd of do-fild

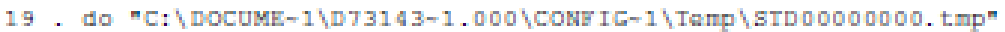

20. pac resido

21 . and of do-f1le

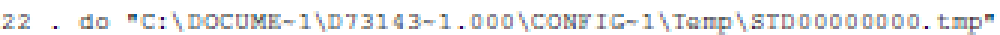

23 . preadict incasos, y (8 missing valuss gensrated)

24 . predict fvar, man

25 . generate upper-1ncasos + $1.96 *$ sqrt. (fvar) (8 missing valuss qunarated)

26 . gunarate 10 wor-1ncasos - $1.96 *$ sqrt. (fvar) (8 missing valuse generated)

27 . twoway (tal1no 1ncasos avetx uppor lowar), titis (Previsao dantro da Anostra)

28 snd of do-f1le

29 . do "C: \DOCUME-1 $\backslash D 73143-1.000 \backslash C O N E I G-1 \backslash$ Temp $\backslash S T D 00000000$. tmp"

30. twoway (tsi1na 1ncasos avetx uppar lowsr), titis (Previsao dentro da Amostra)

31. and of do-fild

32. 
Anexos

Anexo G: Modelo estimado para taxa de mortalidade por "Infarto", metodologia ITSA-ARIMAX

Doutorado Wednesday Decenber 30 15:18:05 2015 Page 1

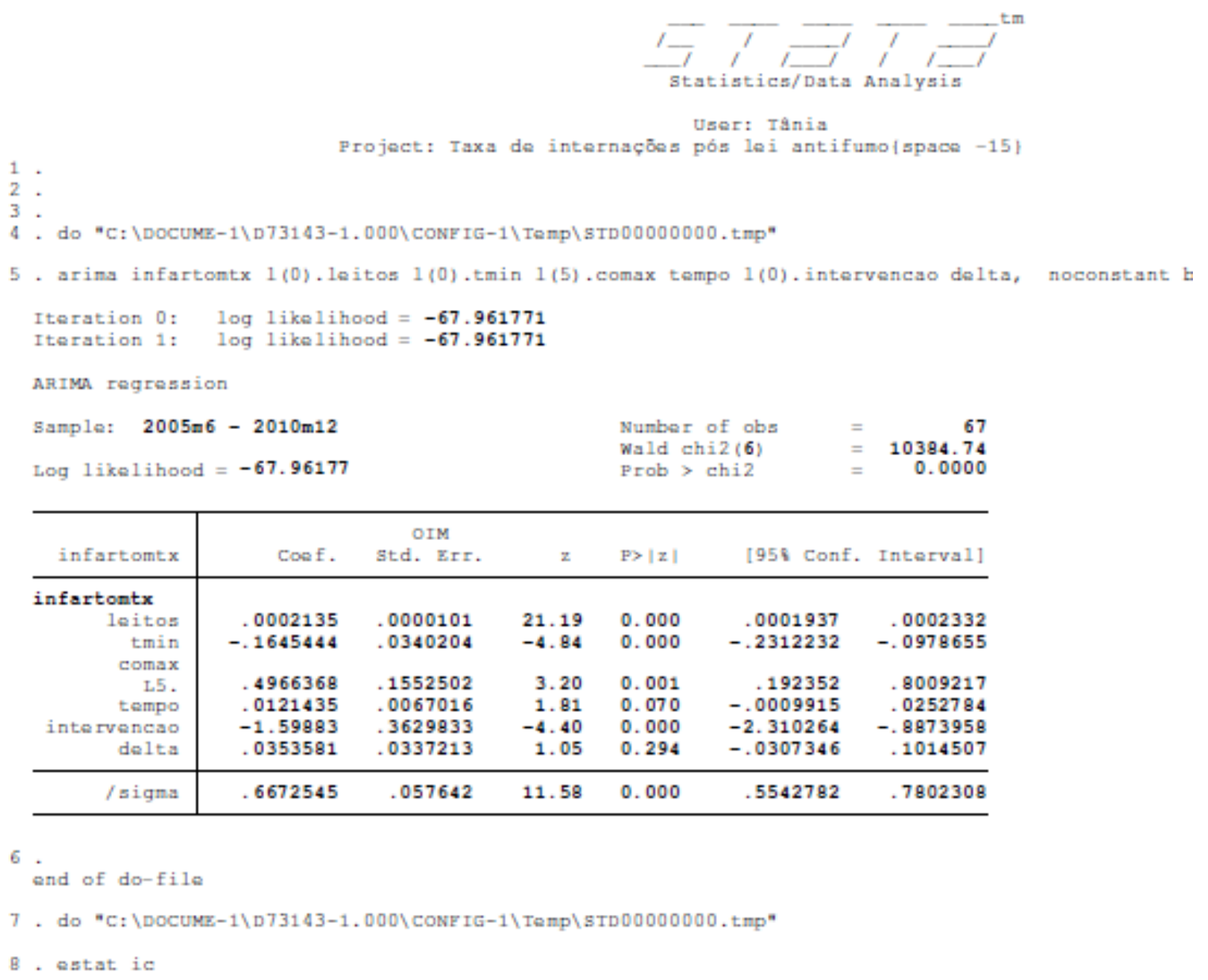

\begin{tabular}{|c|c|c|c|c|c|c|}
\hline Mode 1 & $\mathrm{Obs}$ & 11 (nu11) & 11 (mode 1 ) & $d f$ & AIC & BIC \\
\hline . & 67 & . & -67.96177 & 7 & 149.9235 & 165.3564 \\
\hline
\end{tabular}

Note: $\mathrm{N}=\mathrm{Obs}$ used in calculating BIC; see [R] BIC note

9. estat vce, corr

Correlation matrix of coefficients of arima model

\begin{tabular}{|c|c|c|c|c|c|c|c|}
\hline$\approx(v)$ & $\begin{array}{r}\text { infa-mt:x } \\
\text { leitos }\end{array}$ & tmin & L.5. comax & tempo & interv-o & delta & $\begin{array}{l}\text { sigma } \\
\text { _cons }\end{array}$ \\
\hline $\begin{array}{r}\text { infartontx } \\
\text { leitos } \\
\text { tmin } \\
\text { L5. comax } \\
\text { tempo } \\
\text { intervencao } \\
\text { delta }\end{array}$ & $\begin{array}{r}1.0000 \\
-0.6281 \\
-0.0267 \\
-0.3886 \\
0.0333 \\
0.0452\end{array}$ & $\begin{array}{r}1.0000 \\
-0.6752 \\
-0.2036 \\
0.0899 \\
0.0811\end{array}$ & $\begin{array}{r}1.0000 \\
0.2543 \\
-0.0123 \\
-0.0771\end{array}$ & $\begin{array}{r}1.0000 \\
-0.4228 \\
-0.1964\end{array}$ & $\begin{array}{r}1.0000 \\
-0.6287\end{array}$ & 1.0000 & \\
\hline - cons & -0.0000 & -0.0000 & -0.0000 & 0.0000 & -0.0000 & 0.0000 & 1.0000 \\
\hline
\end{tabular}


10 . predict resido, r

( 5 missing values generated)

11. xcorr infartontx resido, table

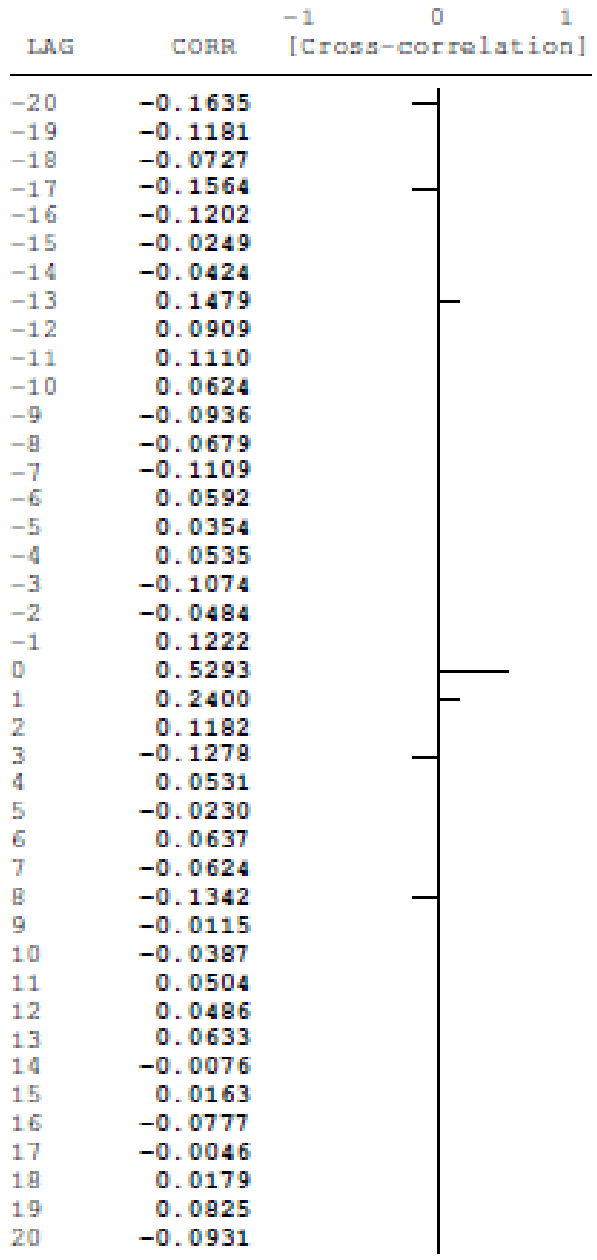

12 . corrgram resido

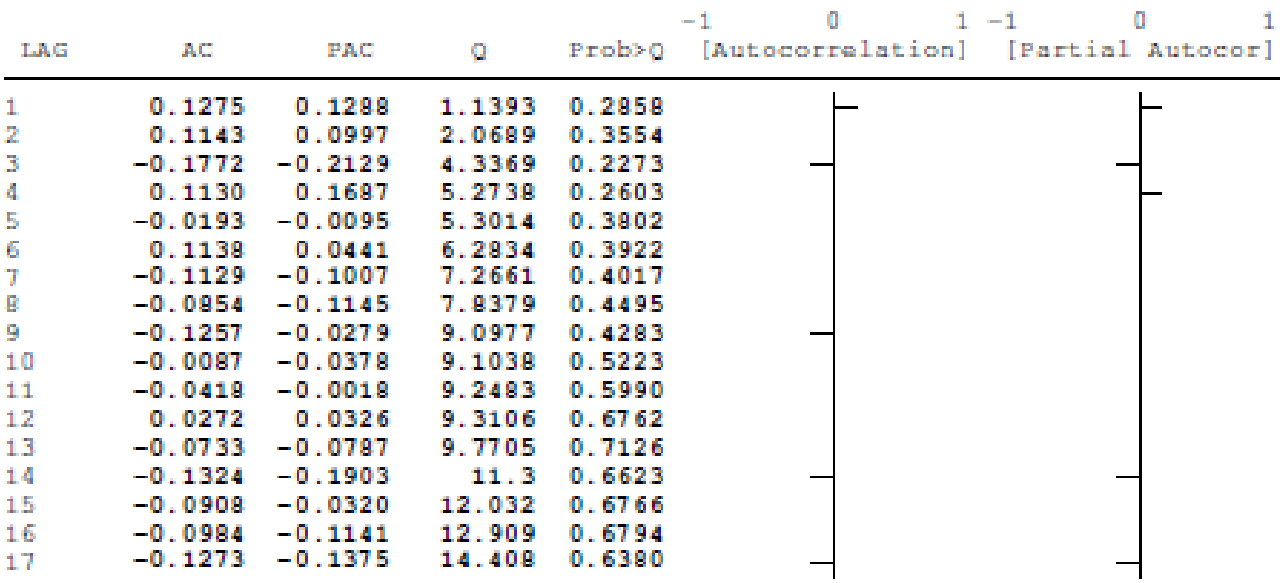




\begin{tabular}{lrrrr|l}
18 & 0.0886 & 0.2784 & 15.148 & 0.6518 \\
19 & 0.0657 & 0.1446 & 15.563 & 0.6862 \\
20 & 0.0115 & -0.0104 & 15.576 & 0.7425 \\
21 & -0.2464 & -0.4366 & 21.676 & 0.4184 \\
22 & -0.0589 & -0.1345 & 22.032 & 0.4580 \\
23 & -0.0214 & -0.1037 & 22.081 & 0.5154 \\
24 & 0.1922 & 0.2069 & 26.05 & 0.3506 \\
25 & -0.0405 & -0.1422 & 26.231 & 0.3953 \\
26 & -0.0473 & -0.1009 & 26.484 & 0.4368 & \\
27 & -0.0236 & 0.1096 & 26.548 & 0.4884 \\
28 & 0.1521 & 0.1746 & 29.291 & 0.3979 & \\
29 & 0.1199 & -0.0352 & 31.04 & 0.3636 & \\
30 & 0.1098 & -0.1234 & 32.545 & 0.3426 & \\
31 & -0.0223 & -0.0594 & 32.609 & 0.3877 &
\end{tabular}

13. pnorm resido

14 . swilk resido

Shapiro-wilk w test for normal data

\begin{tabular}{r|ccccc} 
Variable & obs & w & V & z & Prob>z \\
\hline resido & 67 & 0.96734 & 1.940 & 1.438 & 0.07522
\end{tabular}

15. sum resido

\begin{tabular}{r|rrrrr} 
Variable & obs & Mean & Std. Dev & Min & Max \\
\hline resido & 67 & .0154686 & .6721098 & -1.151714 & 1.788906
\end{tabular}

16.

end of do-file 
Anexos

Anexo $\mathrm{H}$ : Modelo estimado para taxa de mortalidade por "AVC", metodologia ITSA-ARIMAX

Doutorado Wednesday Decenber 30 15:54:40 2015 Page 1

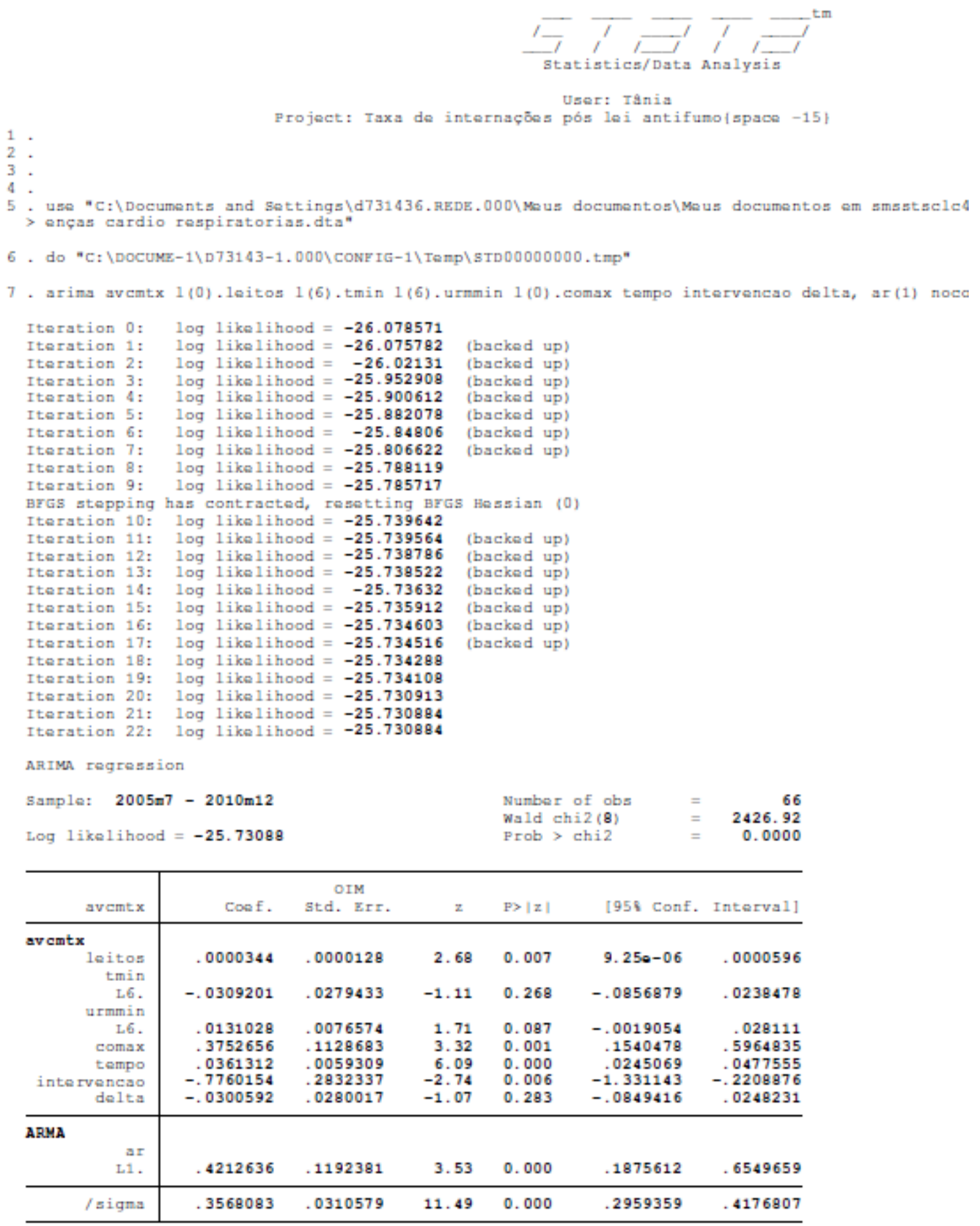


DouToRado Wednezday December 30 15:54:40 2015 Page 2

B.

end of do-file

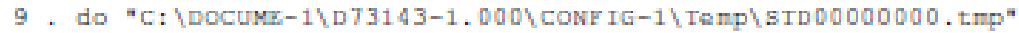

10 .

11 . est.at ic

\begin{tabular}{r|rrrrrr}
\hline Model & obs & 11 (nul1) & 11 (model) & df & AIC & BIC \\
\hline$\cdot$ & 66 & & -25.73088 & 9 & 69.46177 & 89.16866 \\
\hline
\end{tabular}

12 . estat ves, corr

Correlation matrix of coefficients of arima model

\begin{tabular}{|c|c|c|c|c|c|c|c|c|}
\hline$\approx(V)$ & $\begin{array}{l}\text { aventx } \\
\text { leitos }\end{array}$ & 1.6. tmin & L6. urm-n & $\operatorname{cons} x$ & tenpo & interv-o & delta & $\begin{array}{l}\text { ARMA } \\
\text { L.ar }\end{array}$ \\
\hline $\begin{array}{r}\text { avcmtx } \\
\text { leitos } \\
\text { L6. tmin } \\
\text { L6.urmmin } \\
\text { comax } \\
\text { tempo } \\
\text { intervencao } \\
\text { delta }\end{array}$ & $\begin{array}{r}1.0000 \\
-0.5332 \\
-0.5970 \\
-0.0942 \\
-0.3799 \\
0.0243 \\
0.0347\end{array}$ & $\begin{array}{r}1.0000 \\
-0.0799 \\
-0.4850 \\
-0.0696 \\
0.0471 \\
0.1735\end{array}$ & $\begin{array}{r}1.0000 \\
0.0017 \\
0.0440 \\
-0.0070 \\
-0.0803\end{array}$ & $\begin{array}{r}1.0000 \\
0.2175 \\
0.0326 \\
-0.0873\end{array}$ & $\begin{array}{r}1.0000 \\
-0.3542 \\
-0.2977\end{array}$ & $\begin{array}{r}1.0000 \\
-0.5501\end{array}$ & 1.0000 & \\
\hline ARMA & -0.1390 & 0.1728 & -0.0322 & -0.0394 & 0.0599 & 0.1311 & -0.0470 & 1.0000 \\
\hline _cons & 0.0015 & -0.0018 & 0.0003 & 0.0004 & -0.0006 & -0.0014 & 0.0005 & -0.0106 \\
\hline
\end{tabular}

13. predict resido, r

(6 missing values generated)

14 . xcorr avcmtx resido, table

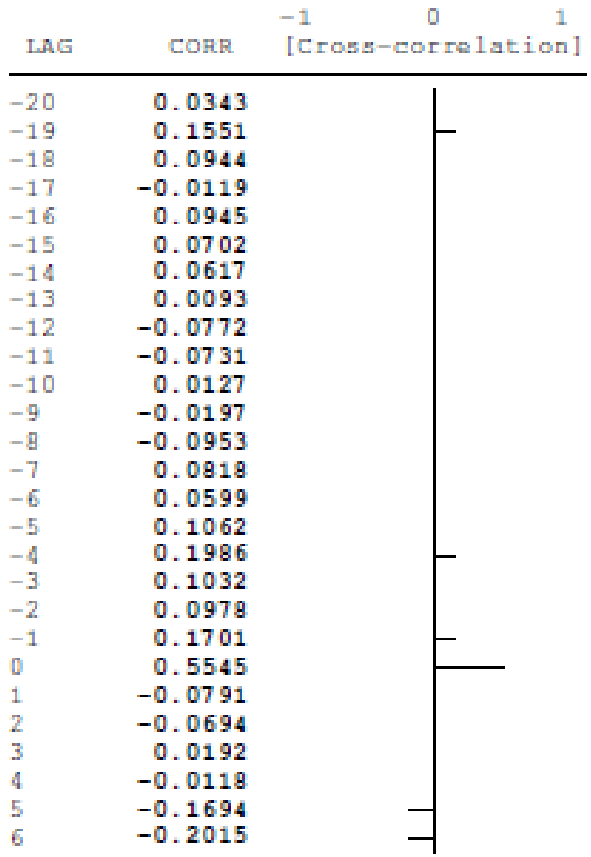


Doutorado Wednesday December 30 15:54:40 2015 Page 3

$\begin{array}{llll}7 & -0.2696 & \\ B & -0.2596 & \\ 9 & -0.2107 & \\ 10 & -0.2250 & \\ 11 & -0.2120 & \\ 12 & -0.1657 & \\ 13 & -0.1061 & \\ 14 & -0.0586 & \\ 15 & -0.0389 & \\ 16 & -0.0557 & \\ 17 & -0.0452 & \\ 18 & 0.0881 & \\ 19 & 0.0665 & \\ 20 & -0.0905 & \end{array}$

15 . corgram resido

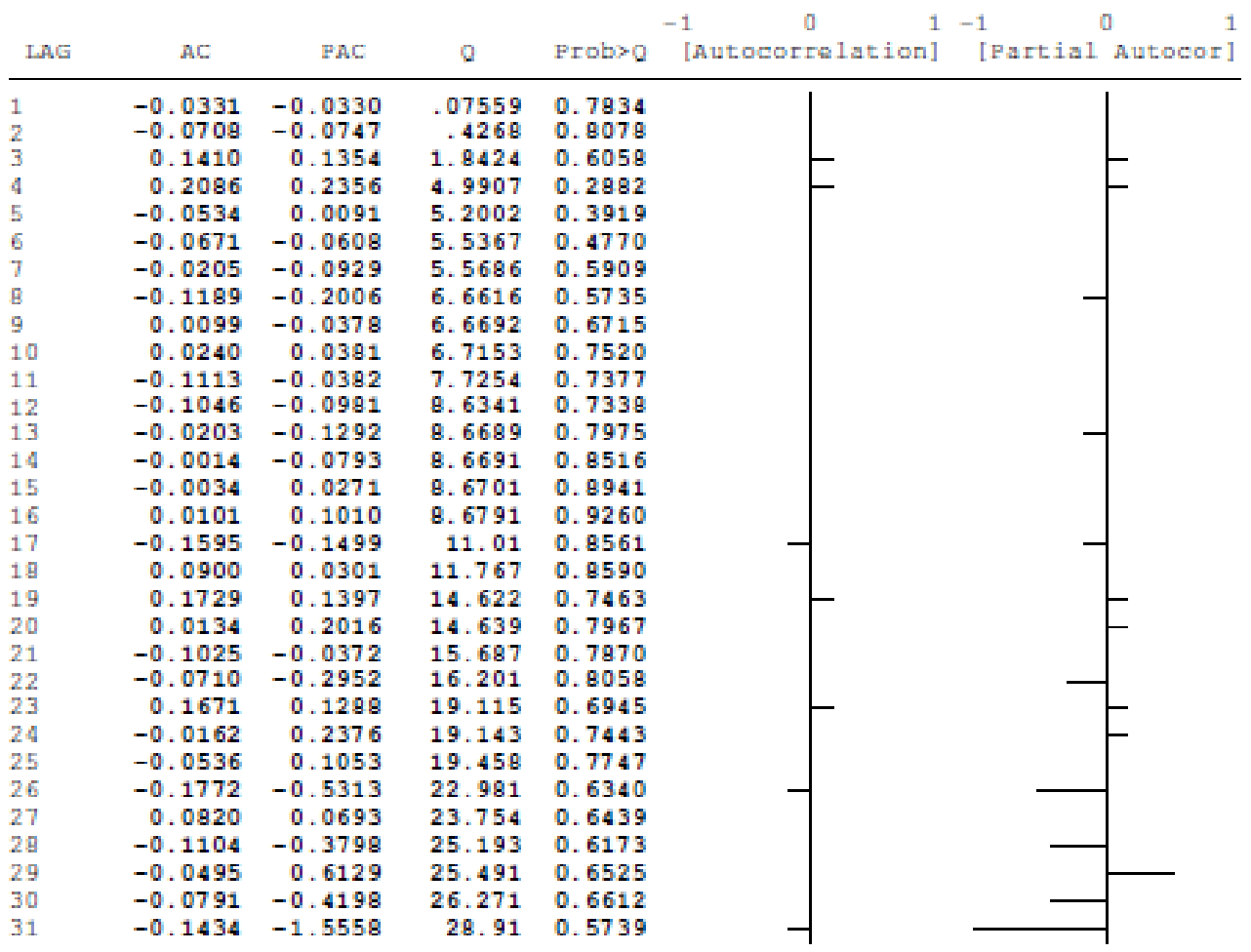

16. pnorm resido

17. swilk resido

Shapiro-wilk w test for normal data

\begin{tabular}{c|ccccc} 
Variable & obs & w & V & z & Prob $>z$ \\
\hline resido & 66 & 0.98653 & 0.790 & -0.510 & 0.69481
\end{tabular}

1B. sum resido

\begin{tabular}{c|ccccc} 
Variable & Obs & Mean & Std. Dev. & Min & Max \\
\hline resido & 66 & .0068651 & .3618553 & -.7928735 & 1.074152
\end{tabular}

19.

end of do-file 


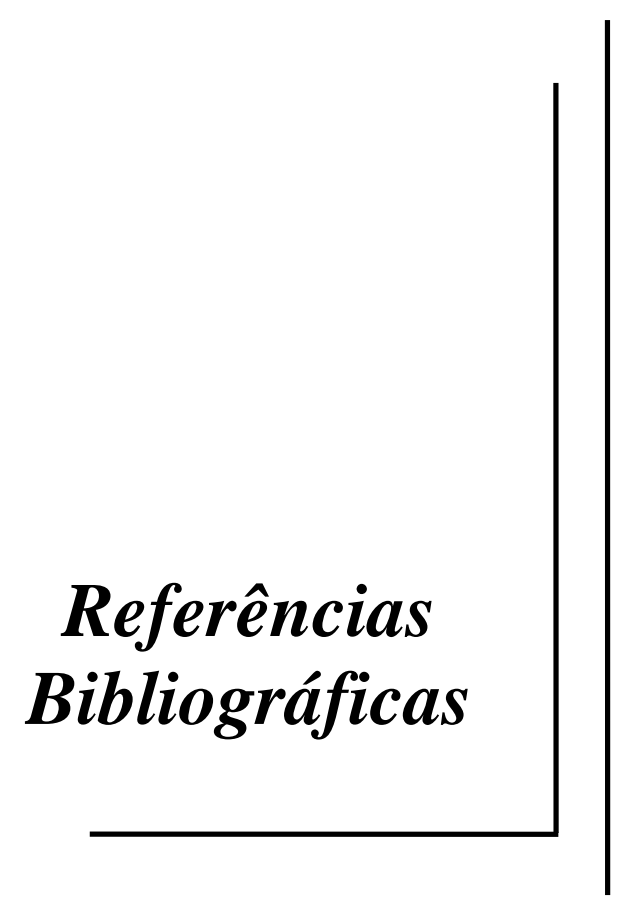




\section{REFERÊNCIAS BIBLIOGRÁFICAS}

1. Saúde Md. tabnet.datasus.gov.br/cgi/sim/Consolida_Sim_2011.pdf. 2011 [updated 2011; cited 201517 june]; Available from.

2. de Souza Mde F, Alencar AP, Malta DC, Moura L, Mansur Ade P. Serial temporal analysis of ischemic heart disease and stroke death risk in five regions of Brazil from 1981 to 2001. Arq Bras Cardiol. 2006 Dec;87(6):735-40.

3. Silva MAD, A.G.M.R. Sousa and Schargodsky. Fatores de risco para infarto do miocárdio no Brasil - Estudo FRICAS. Arq Bras Cardiol. 1998;71(5):677-75.

4. Tyden P, Hansen O, Engstrom G, Hedblad B, Janzon L. Myocardial infarction in an urban population: worse long term prognosis for patients from less affluent residential areas. J Epidemiol Community Health. 2002 Oct;56(10):785-90.

5. Wamala SP, Lynch J, Kaplan GA. Women's exposure to early and later life socioeconomic disadvantage and coronary heart disease risk: the Stockholm Female Coronary Risk Study. Int J Epidemiol. 2001 Apr;30(2):275-84.

6. Cooper R, Cutler J, Desvigne-Nickens P, Fortmann SP, Friedman L, Havlik R, et al. Trends and disparities in coronary heart disease, stroke, and other cardiovascular diseases in the United States: findings of the national conference on cardiovascular disease prevention. Circulation. 2000 Dec 19;102(25):3137-47.

7. Philbin EF, McCullough PA, DiSalvo TG, Dec GW, Jenkins PL, Weaver WD. Socioeconomic status is an important determinant of the use of invasive procedures after acute myocardial infarction in New York State. Circulation. 2000 Nov 7;102(19 Suppl 3):III107-15.

8. Szwarcwald CL, Bastos FI, Esteves MA, de Andrade CL, Paez MS, Medici EV, et al. [Income inequality and health: the case of Rio de Janeiro]. Cad Saude Publica. 1999 JanMar;15(1):15-28.

9. Mansur AP, M.F.M. Souza, et al. Trends of the risk of death due to circulatory, cerebrovascular and ischemic heart diseases in 11 brazilian capitals from 1980 to 1998. Arq Bras Cardiol. 2002;79(3):277-84.

10. Hatsukami DK, Stead LF, Gupta PC. Tobacco addiction. Lancet. 2008 Jun 14;371(9629):2027-38.

11. Doll R, Peto R, Boreham J, Sutherland I. Mortality in relation to smoking: 50 years' observations on male British doctors. BMJ. 2004 Jun 26;328(7455):1519.

12. Meyers DG, Neuberger JS. Cardiovascular effect of bans on smoking in public places. Am J Cardiol. 2008 Nov 15;102(10):1421-4.

13. Husten CG, S.L. Thorne. Tobacco: health effects and control. 15th ed. Wallace RB, N. Kohatsu, editor. New York: McGraw Hill; 2008.

14. Services USDoHaH. The Health Consequences of Involuntary Exposure to Tobacco Smoke. A Report of the Surgeon General. . In: U.S. Department of Health and Human Services CfDCaP, office of smoking and health., editor. Atlanta; 2006. p. 1-15.

15. Law MR, Morris JK, Wald NJ. Environmental tobacco smoke exposure and ischaemic heart disease: an evaluation of the evidence. BMJ. 1997 Oct 18;315(7114):97380 . 
16. He J, Vupputuri S, Allen K, Prerost MR, Hughes J, Whelton PK. Passive smoking and the risk of coronary heart disease--a meta-analysis of epidemiologic studies. N Engl J Med. 1999 Mar 25;340(12):920-6.

17. Barnoya J, Glantz SA. Cardiovascular effects of secondhand smoke: nearly as large as smoking. Circulation. 2005 May 24;111(20):2684-98.

18. Thun M, Henley J, Apicella L. Epidemiologic studies of fatal and nonfatal cardiovascular disease and ETS exposure from spousal smoking. Environ Health Perspect. 1999 Dec;107 Suppl 6:841-6.

19. Bonita R, Duncan J, Truelsen T, Jackson RT, Beaglehole R. Passive smoking as well as active smoking increases the risk of acute stroke. Tob Control. 1999 Summer;8(2):156-60.

20. Whincup PH, Gilg JA, Emberson JR, Jarvis MJ, Feyerabend C, Bryant A, et al. Passive smoking and risk of coronary heart disease and stroke: prospective study with cotinine measurement. BMJ. 2004 Jul 24;329(7459):200-5.

21. Glantz SA, Parmley WW. Passive smoking and heart disease. Mechanisms and risk. JAMA. 1995 Apr 5;273(13):1047-53.

22. Howard G, Thun MJ. Why is environmental tobacco smoke more strongly associated with coronary heart disease than expected? A review of potential biases and experimental data. Environ Health Perspect. 1999 Dec;107 Suppl 6:853-8.

23. Law MR, Wald NJ. Environmental tobacco smoke and ischemic heart disease. Prog Cardiovasc Dis. 2003 Jul-Aug;46(1):31-8.

24. Pechacek TF, Babb S. How acute and reversible are the cardiovascular risks of secondhand smoke? BMJ. 2004 Apr 24;328(7446):980-3.

25. Otsuka R, Watanabe H, Hirata K, Tokai K, Muro T, Yoshiyama M, et al. Acute effects of passive smoking on the coronary circulation in healthy young adults. JAMA. $2001 \mathrm{Jul} 25 ; 286(4): 436-41$.

26. Dinno A, Glantz S. Clean indoor air laws immediately reduce heart attacks. Prev Med. 2007 Jul;45(1):9-11.

27. Raupach T, Schafer K, Konstantinides S, Andreas S. Secondhand smoke as an acute threat for the cardiovascular system: a change in paradigm. Eur Heart J. 2006 Feb;27(4):386-92.

28. Vasselli S, Papini P, Gaelone D, Spizzichino L, De Campora E, Gnavi R, et al. Reduction incidence of myocardial infarction associated with a national legislative ban on smoking. Minerva Cardioangiol. 2008 Apr;56(2):197-203.

29. Decreto n. 5.658, de 2 de janeiro de 2006. Promulga a convenção-quadro sobre o Controle do uso do Tabaco. In: Legislativa A, editor. Brasilia-DF: Diario Oficial da Uniao; 2006. p. 1.

30. Correa PCRP, S.M. Barreto, et al. Metodos de estimativa da mortalidade atribuível ao tabagismo: uma revisão da literatura. Epidemiol Serv Saude. 2008;17(1):43-57.

31. Bartecchi C, Alsever RN, Nevin-Woods C, Thomas WM, Estacio RO, Bartelson $\mathrm{BB}$, et al. Reduction in the incidence of acute myocardial infarction associated with a citywide smoking ordinance. Circulation. 2006 Oct 3;114(14):1490-6.

32. Khuder SA, Milz S, Jordan T, Price J, Silvestri K, Butler P. The impact of a smoking ban on hospital admissions for coronary heart disease. Prev Med. 2007 Jul;45(1):3-8. 
33. Juster HR, Loomis BR, Hinman TM, Farrelly MC, Hyland A, Bauer UE, et al. Declines in hospital admissions for acute myocardial infarction in New York state after implementation of a comprehensive smoking ban. Am J Public Health. 2007 Nov;97(11):2035-9.

34. Sargent RP, Shepard RM, Glantz SA. Reduced incidence of admissions for myocardial infarction associated with public smoking ban: before and after study. BMJ. 2004 Apr 24;328(7446):977-80.

35. Sims M, Maxwell R, Bauld L, Gilmore A. Short term impact of smoke-free legislation in England: retrospective analysis of hospital admissions for myocardial infarction. BMJ.340:c2161.

36. Pell JP, Haw S, Cobbe S, Newby DE, Pell AC, Fischbacher C, et al. Smoke-free legislation and hospitalizations for acute coronary syndrome. N Engl J Med. $2008 \mathrm{Jul}$ 31;359(5):482-91.

37. Edwards EBC, D. O'Dea, et al. After the smoke has cleared: evaluation of the impact of a new smoke free law. In: Health NZMo, editor.; 2006.

38. Barone-Adesi F, Vizzini L, Merletti F, Richiardi L. Short-term effects of Italian smoking regulation on rates of hospital admission for acute myocardial infarction. Eur Heart J. 2006 Oct;27(20):2468-72.

39. Lemstra M, Neudorf C, Opondo J. Implications of a public smoking ban. Can J Public Health. 2008 Jan-Feb;99(1):62-5.

40. Cesaroni G, Forastiere F, Agabiti N, Valente P, Zuccaro P, Perucci CA. Effect of the Italian smoking ban on population rates of acute coronary events. Circulation. 2008 Mar 4;117(9):1183-8.

41. Mulcahy M, Evans DS, Hammond SK, Repace JL, Byrne M. Secondhand smoke exposure and risk following the Irish smoking ban: an assessment of salivary cotinine concentrations in hotel workers and air nicotine levels in bars. Tob Control. 2005 Dec;14(6):384-8.

42. Reduced hospitalizations for acute myocardial infarction after implementation of a smoke-free ordinance--City of Pueblo, Colorado, 2002-2006. MMWR Morb Mortal Wkly Rep. 2009 Jan 2;57(51):1373-7.

43. McNabola A, Broderick B, Johnston P, Gill L. Effects of the smoking ban on benzene and 1,3-butadiene levels in pubs in Dublin. J Environ Sci Health A Tox Hazard Subst Environ Eng. 2006;41(5):799-810.

44. Mulcahy M, D.S. Evans, et al. How does the Irish smoking ban measure up? A before and after study of particle concentrations in Irish pubs. Indoor Air. 2005;15:28-9.

45. Sebrie EM, Sandoya E, Hyland A, Bianco E, Glantz SA, Cummings KM. Hospital admissions for acute myocardial infarction before and after implementation of a comprehensive smoke-free policy in Uruguay. Tob Control. May;22(e1):e16-20.

46. Goncalves HS. Antitabagismo no Brasil: da mobilização da comunidade médica à política de saúde pública (1950-1986). . Belo Horizonte: Universidade Federal de Minas Gerais; 2009.

47. Torres-Homem. O Abuso do Tabaco como causa de angina do peito. Gazeta Medica do Rio de Janeiro. 1863:15.

48. Legislação sobre tabagismo no estado de São Paulo: proposta de lei municipal padrão. . Monografia. Sao Paulo: Monografia - Comissão Estadual de Prevenção e 
Controle do Tabagismo - Centro de Vigilância Epidemiológica Prof. Alexandre Vranjac; 1997.

49. Silveira J, J.S. Neves, et al. Carta de Salvador: O tabagismo. Um novo desafio. JBM. 1979;36:62-8.

50. Rosemberg J. Tabagismo. Serio problema de saude publica. 2a ed ed. Sao Paulo: Ed Almed Edusp; 1988.

51. Tabagismo: sistema de radiopostos. Monografia - Secretaria de Estado da Educação. Sao Paulo; 1980.

52. Issa JS, Abe TM, Pereira AC, Megid MC, Shimabukuro CE, Valentin LS, et al. The effect of Sao Paulo's smoke-free legislation on carbon monoxide concentration in hospitality venues and their workers. Tob Control. Mar;20(2):156-62.

53. Jarvis MJ, Belcher M, Vesey C, Hutchison DC. Low cost carbon monoxide monitors in smoking assessment. Thorax. 1986 Nov;41(11):886-7.

54. Wald NJ, Idle M, Boreham J, Bailey A. Carbon monoxide in breath in relation to smoking and carboxyhaemoglobin levels. Thorax. 1981 May;36(5):366-9.

55. Burnett RT, Cakmak S, Raizenne ME, Stieb D, Vincent R, Krewski D, et al. The association between ambient carbon monoxide levels and daily mortality in Toronto, Canada. J Air Waste Manag Assoc. 1998 Aug;48(8):689-700.

56. Crawford VL, McCann M, Stout RW. Changes in seasonal deaths from myocardial infarction. QJM. 2003 Jan;96(1):45-52.

57. Douglas AS, Dunnigan MG, Allan TM, Rawles JM. Seasonal variation in coronary heart disease in Scotland. J Epidemiol Community Health. 1995 Dec;49(6):575-82.

58. Kloner RA. The "Merry Christmas Coronary" and "Happy New Year Heart Attack" phenomenon. Circulation. 2004 Dec 21;110(25):3744-5.

59. Pell JP, Cobbe SM. Seasonal variations in coronary heart disease. QJM. 1999 Dec;92(12):689-96.

60. Seo DC, Torabi MR. Reduced admissions for acute myocardial infarction associated with a public smoking ban: matched controlled study. J Drug Educ. 2007;37(3):217-26.

61. Bonetti PO, Trachsel LD, Kuhn MU, Schulzki T, Erne P, Radovanovic D, et al. Incidence of acute myocardial infarction after implementation of a public smoking ban in Graubunden, Switzerland: two year follow-up. Swiss Med Wkly.141:w13206.

62. Brook RD, Franklin B, Cascio W, Hong Y, Howard G, Lipsett M, et al. Air pollution and cardiovascular disease: a statement for healthcare professionals from the Expert Panel on Population and Prevention Science of the American Heart Association. Circulation. 2004 Jun 1;109(21):2655-71.

63. Sun Q, Hong X, Wold LE. Cardiovascular effects of ambient particulate air pollution exposure. Circulation. Jun 29;121(25):2755-65.

64. IBGE. Sinopse do censo demográfico 2010 Brasil. 2010.

65. Boletim Eletrônico CEInfo. In: saúde Sd, editor. Sao Paulo; 2010.

66. Helfenstein U. Box-Jenkins modelling in medical research. Stat Methods Med Res. 1996 Mar;5(1):3-22.

67. McDowell A. From the help desk: Transfer functions. . Stata Journal. 2002;2(1):71-

85.

68. Linden A. Conducting interrupted time series analysis for single and multiple group comparisons. Stata Journal. 2015;15(2):480-500. 
69. Masi Ed. Análise de intervenção em séries temporais de dengue e leptospirose da cidade de São Paulo: influência de fatores políticos, administrativos, técnicos e ambientais. Sao Paulo: USP; 2014.

70. Becketti S. Introduction to Time Series Using Stata. . College Station, TX: Stata Press; 2013.

71. Reduced secondhand smoke exposure after implementation of a comprehensive statewide smoking ban--New York, June 26, 2003-June 30, 2004. MMWR Morb Mortal Wkly Rep. 2007 Jul 20;56(28):705-8.

72. Loomis BR, Juster HR. Association of indoor smoke-free air laws with hospital admissions for acute myocardial infarction and stroke in three states. J Environ Public Health.2012:589018.

73. Cox B, Vangronsveld J, Nawrot TS. Impact of stepwise introduction of smoke-free legislation on population rates of acute myocardial infarction deaths in Flanders, Belgium. Heart. Sep 15;100(18):1430-5.

74. Stallings-Smith S, Zeka A, Goodman P, Kabir Z, Clancy L. Reductions in cardiovascular, cerebrovascular, and respiratory mortality following the national irish smoking ban: interrupted time-series analysis. PLoS One. 2013;8(4):e62063.

75. Thach TQ, McGhee SM, So JC, Chau J, Chan EK, Wong CM, et al. The smoke-free legislation in Hong Kong: its impact on mortality. Tob Control. Nov 19.

76. Aguero F, Degano IR, Subirana I, Grau M, Zamora A, Sala J, et al. Impact of a partial smoke-free legislation on myocardial infarction incidence, mortality and casefatality in a population-based registry: the REGICOR Study. PLoS One.8(1):e53722.

77. Mackay DF, Haw S, Newby DE, Langhorne P, Lloyd SM, McConnachie A, et al. Impact of Scotland's comprehensive, smoke-free legislation on stroke. PLoS One.8(5):e62597.

78. Conceicao GM, Miraglia SG, Kishi HS, Saldiva PH, Singer JM. Air pollution and child mortality: a time-series study in Sao Paulo, Brazil. Environ Health Perspect. 2001 Jun;109 Suppl 3:347-50.

79. Daumas RP, Mendonca GA, Ponce de Leon A. [Air pollution and mortality in the elderly in Rio de Janeiro: a time-series analysis]. Cad Saude Publica. 2004 JanFeb;20(1):311-9.

80. Gouveia N, Fletcher T. Time series analysis of air pollution and mortality: effects

by cause, age and socioeconomic status. J Epidemiol Community Health. 2000 Oct;54(10):750-5.

81. Stieb DM, Judek S, Burnett RT. Meta-analysis of time-series studies of air pollution and mortality: effects of gases and particles and the influence of cause of death, age, and season. J Air Waste Manag Assoc. 2002 Apr;52(4):470-84.

82. Gouveia N, de Freitas CU, Martins LC, Marcilio IO. [Respiratory and cardiovascular hospitalizations associated with air pollution in the city of Sao Paulo, Brazil]. Cad Saude Publica. 2006 Dec;22(12):2669-77.

83. Vigitel 2007. In: Saude Md, editor. Brasilia: Ministerio da Saude; 2009.

84. Vigitel 2008. In: Saude Md, editor. Brasilia: Ministerio da Saude; 2009.

85. Vigitel 2009. In: Saude Md, editor. Brasilia: Ministerio da Saude; 2010.

86. Vigitel 2010. In: Saude Md, editor. Brasilia: Ministerio da Saude; 2011.

87. Vigitel 2011. In: Saúde Md, editor. Brasília: Ministerio da Saude; 2012. 


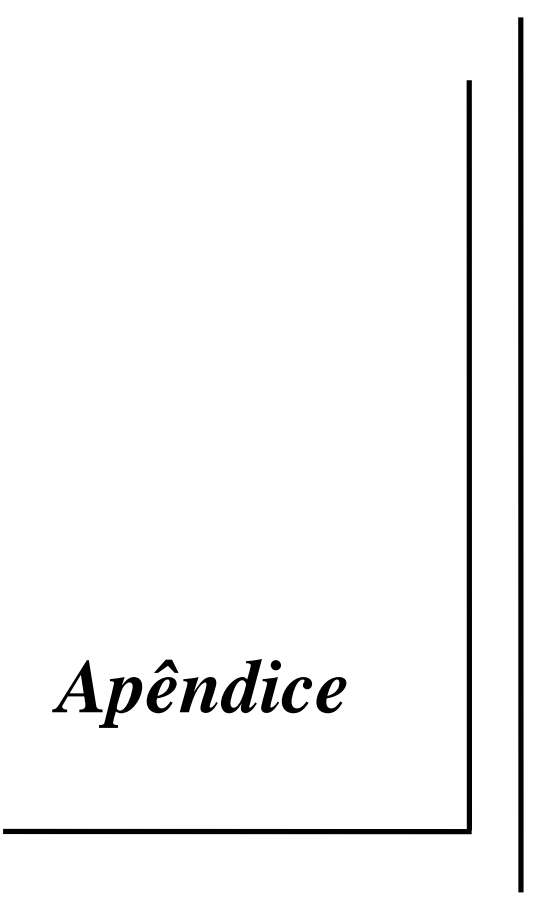




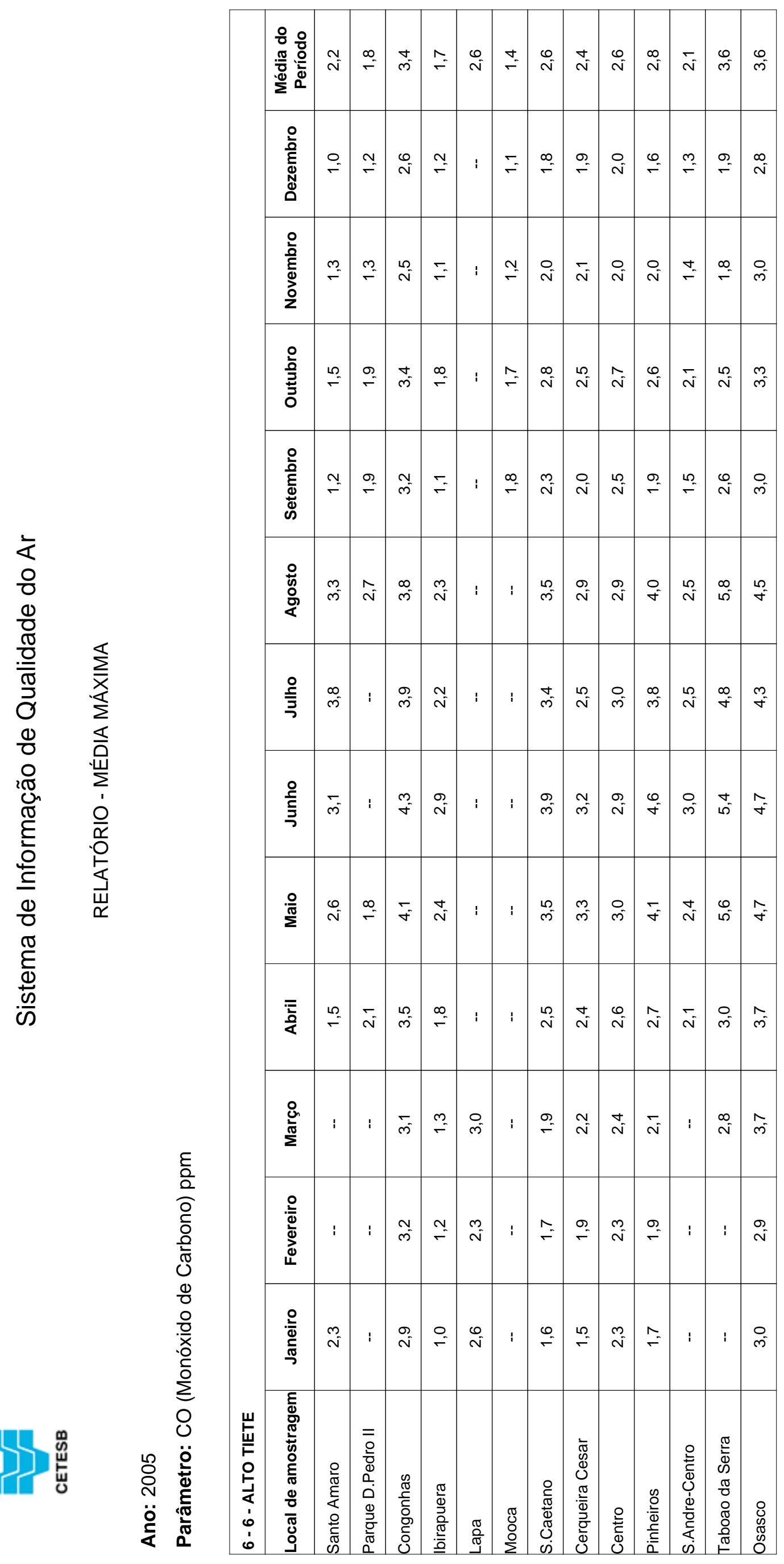




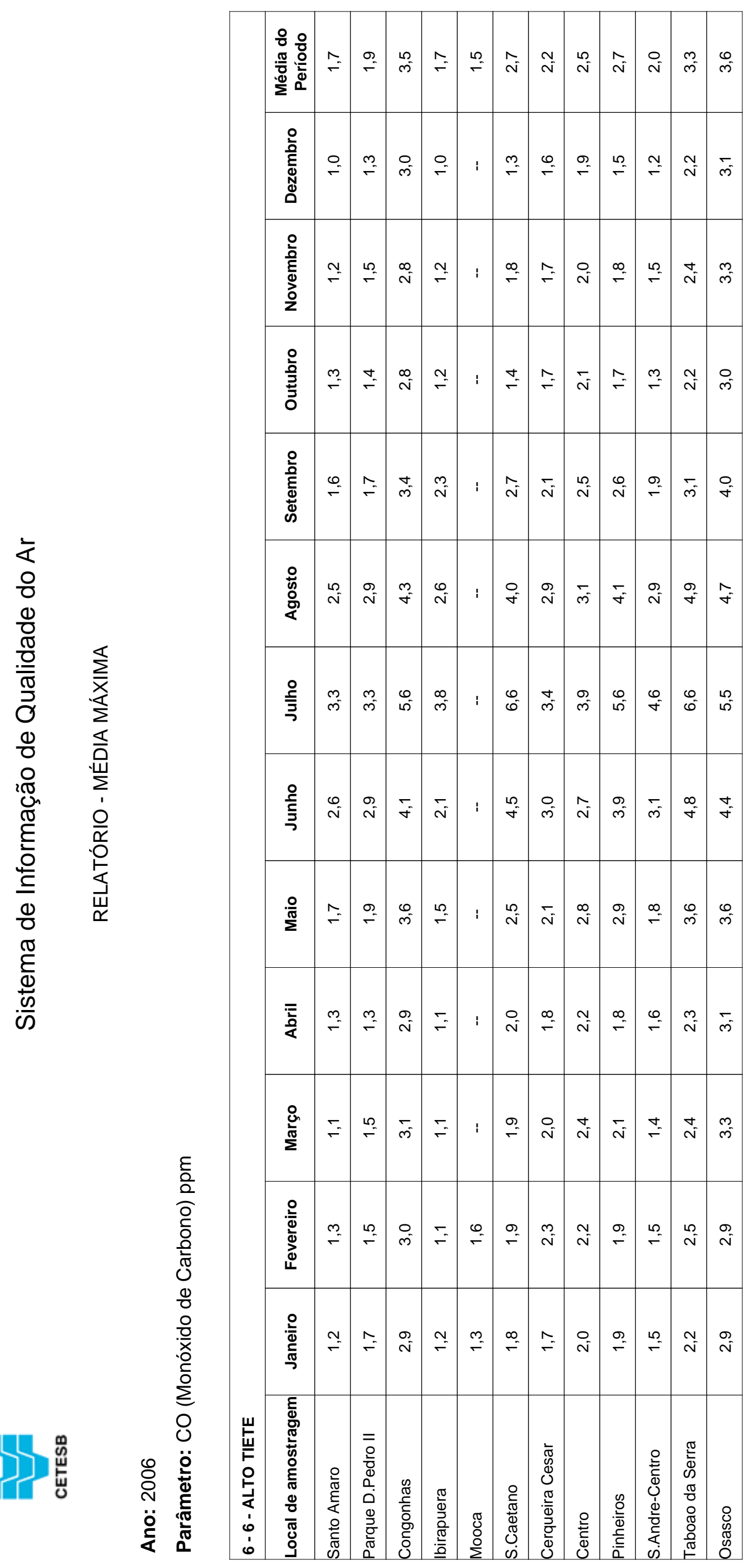




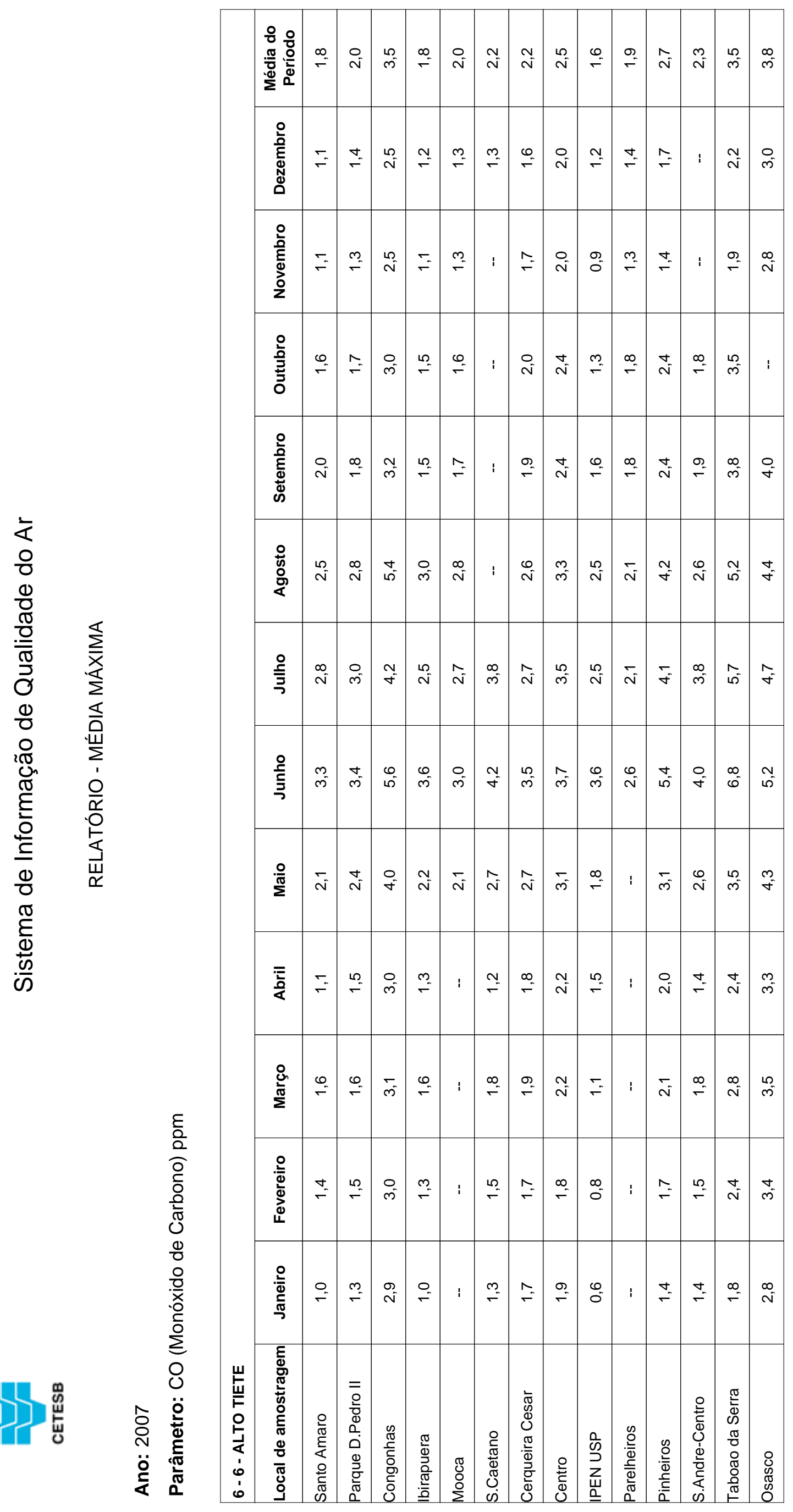




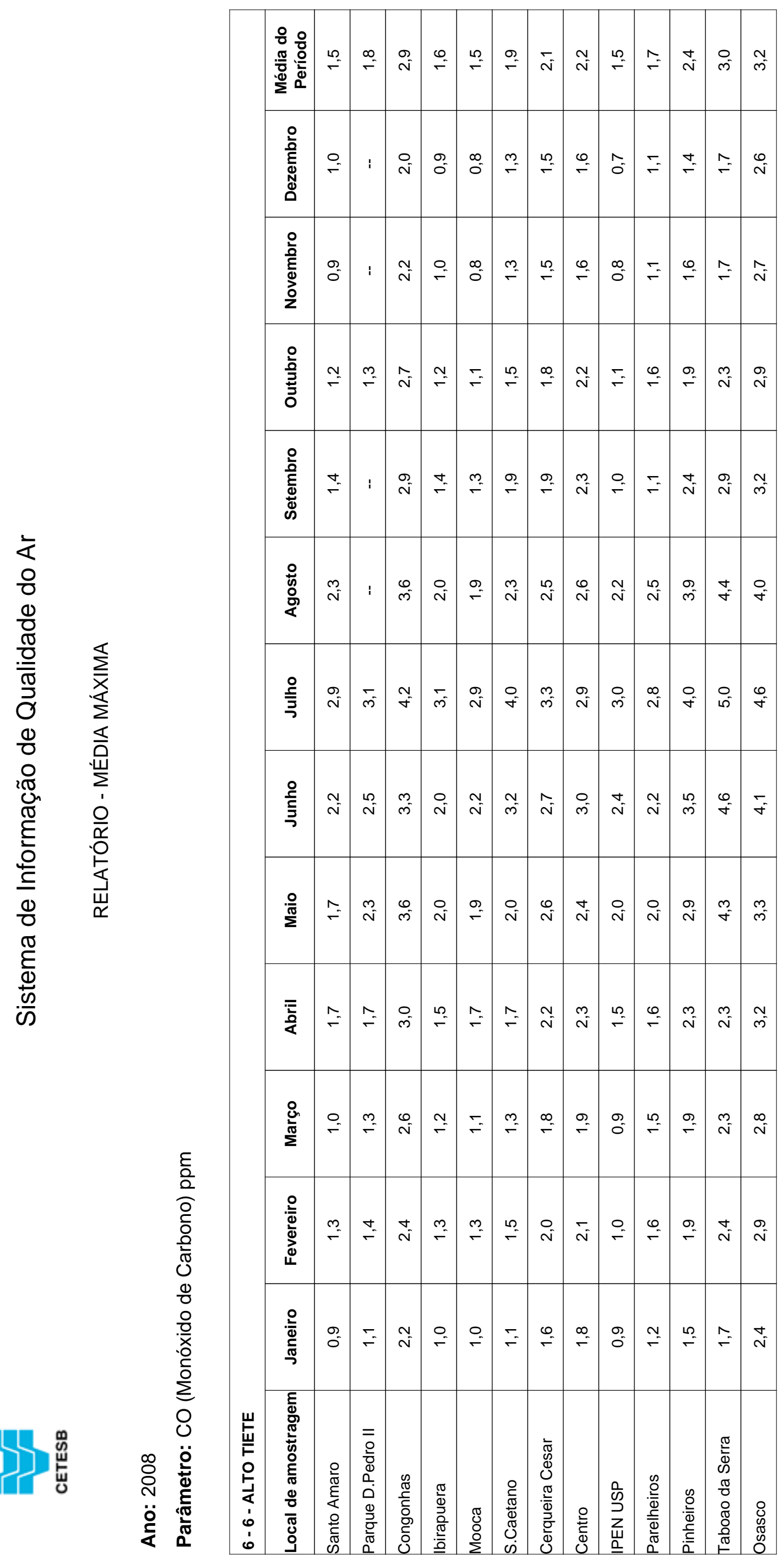




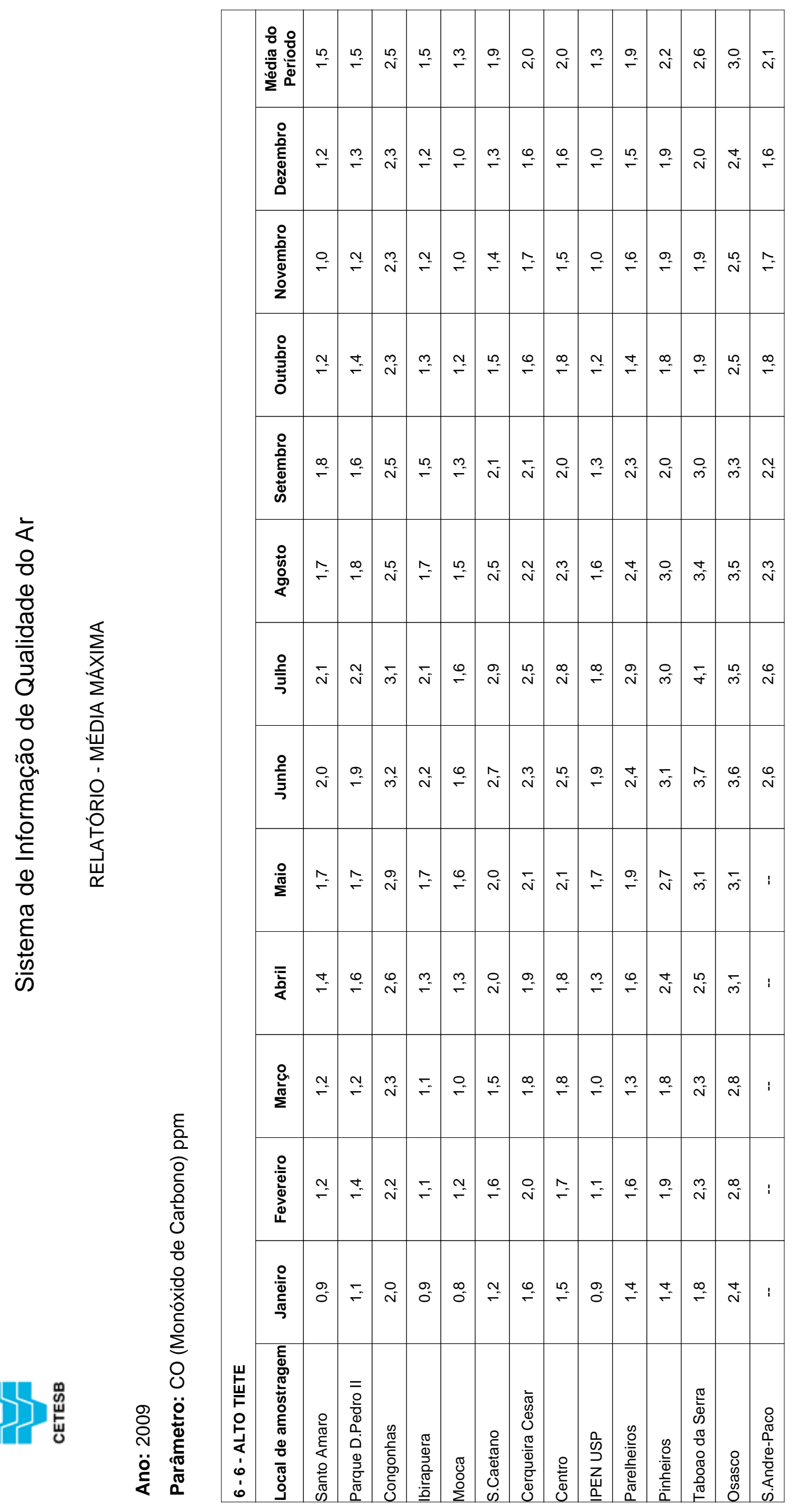




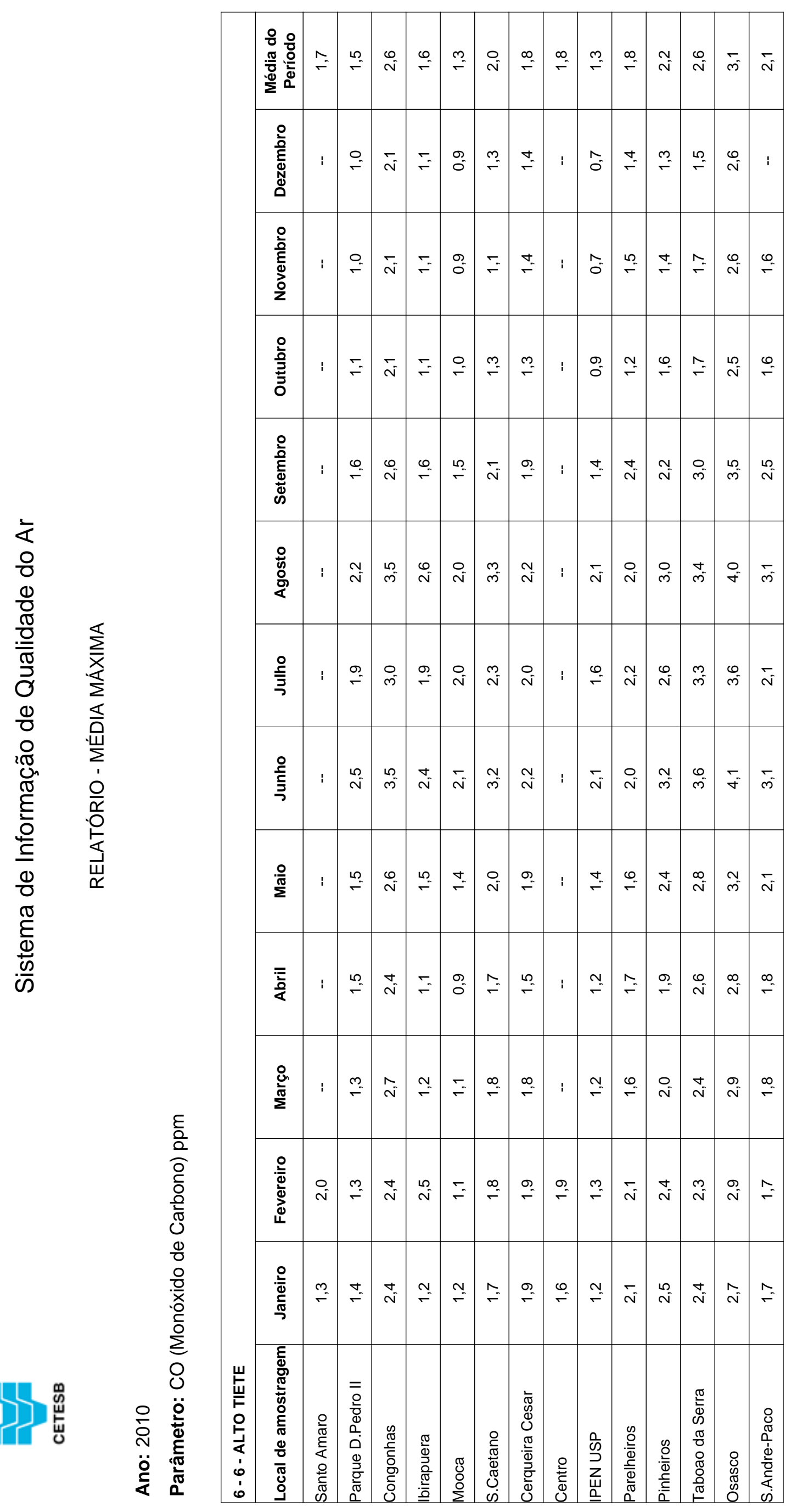




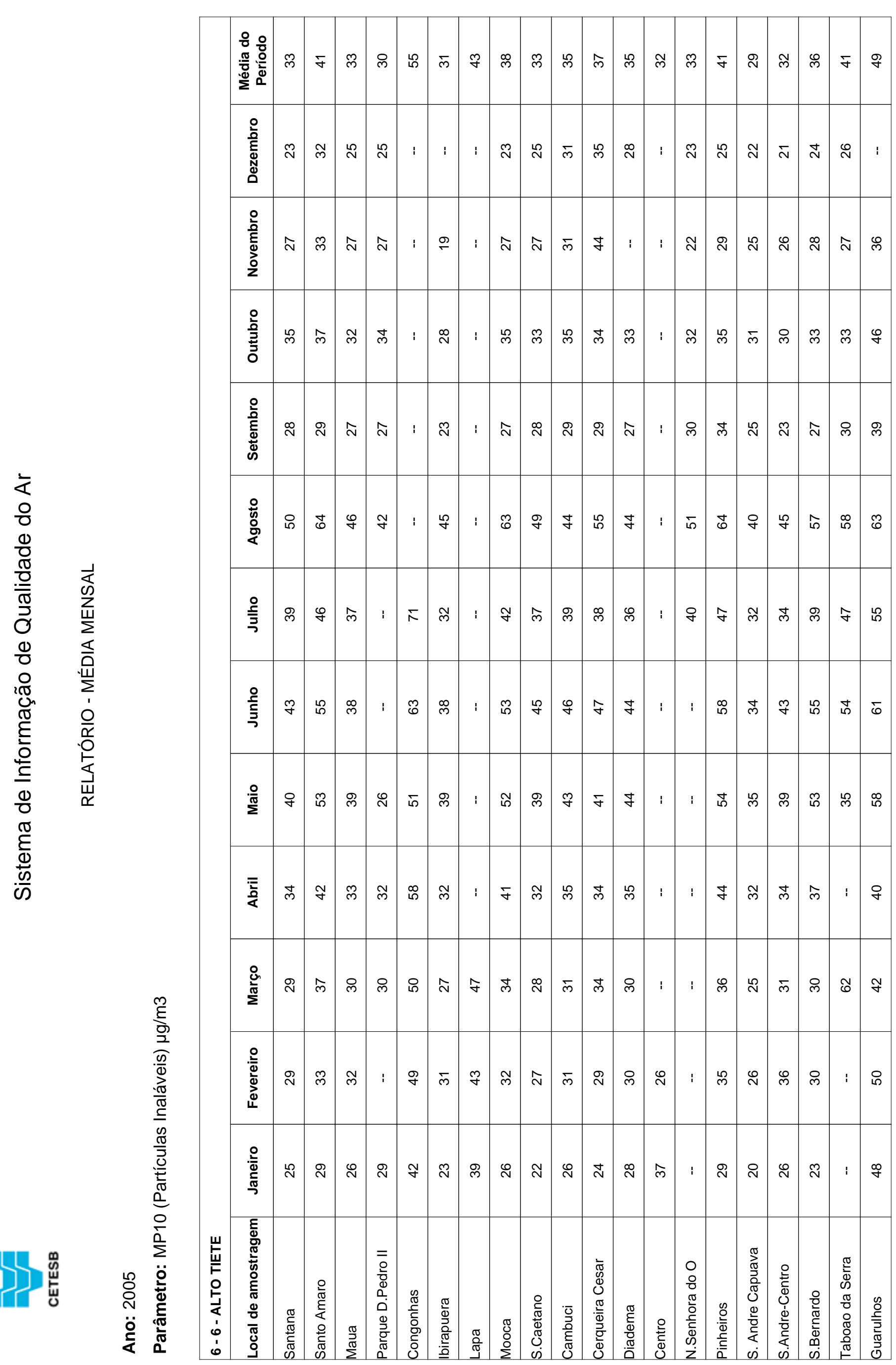




\begin{tabular}{|c|c|c|c|c|c|c|c|c|c|c|c|c|c|c|c|c|c|c|c|c|}
\hline 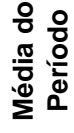 & ల్ల & $\bar{\gamma}$ & ల్ల & $\bar{\gamma}$ & in & $\stackrel{\infty}{m}$ & ले & లి & ల్లి & $\ddot{m}$ & ले & $\hat{m}$ & 邑 & q & ల్ల & 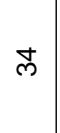 & $\hat{m}$ & $\hat{m}$ & $\hat{\theta}$ & ᄂ \\
\hline 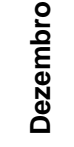 & $\approx$ & $\stackrel{\infty}{\sim}$ & $\stackrel{d}{N}$ & $\stackrel{\sim}{N}$ & $\stackrel{\wp}{\circ}$ & $\stackrel{d}{N}$ & : & $\stackrel{\infty}{\sim}$ & $\hat{N}$ & లి & $\stackrel{N}{\sim}$ & ల్ల & $\stackrel{d}{N}$ & న్ & $\hat{N}$ & జ & $\hat{N}$ & $\stackrel{\leftrightarrow}{\sim}$ & : & ले \\
\hline 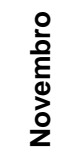 & $\stackrel{\llcorner}{\sim}$ & है & $\stackrel{\mathbb{N}}{ }$ & ৪্ল & 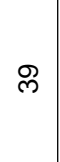 & $\stackrel{\sim}{N}$ & : & ল & ৪ & ల్ల & ని & $\hat{m}$ & $\hat{N}$ & $\stackrel{N}{\sim}$ & $\stackrel{\llcorner}{\sim}$ & $\stackrel{\sim}{N}$ & ल & $\stackrel{\infty}{N}$ & : & @ \\
\hline 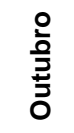 & ల & ஓ & $\hat{N}$ & ল্ & F & $\stackrel{\infty}{N}$ & i & 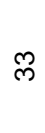 & ' & ల్ల & $\stackrel{\infty}{N}$ & $\hat{m}$ & I & $\hat{N}$ & $\stackrel{\sim}{\sim}$ & $\hat{N}$ & న & $\stackrel{d}{N}$ & F & স্ \\
\hline 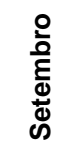 & $\stackrel{\infty}{\infty}$ & F & $\ddot{ల}$ & 寸 & 요 & P & : & ঙ̊ & ల్ల & স্ & 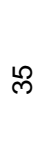 & F & $\ddot{ల}$ & F & ले & $\hat{m}$ & $\hat{\text { m }}$ & $\hat{m}$ & $\widetilde{్}$ & f \\
\hline 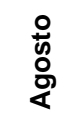 & $\stackrel{\infty}{q}$ & in & \% & $\stackrel{\infty}{\cap}$ & ธิ & is & : & กิ & is & भ & \& & : & f & 吕 & ๆ & g & in & f & 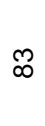 & 욤 \\
\hline
\end{tabular}

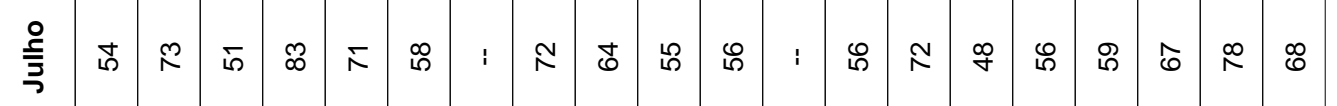

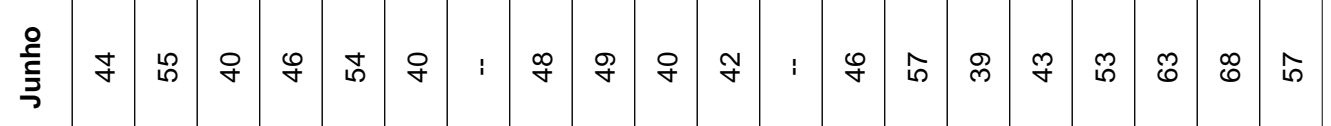

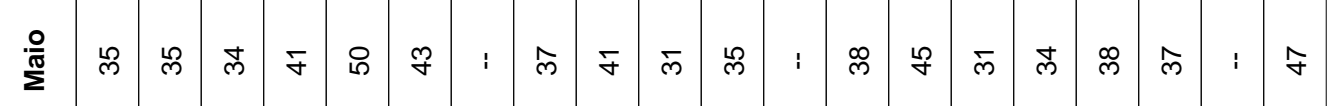

$\overline{\bar{c}}$

总

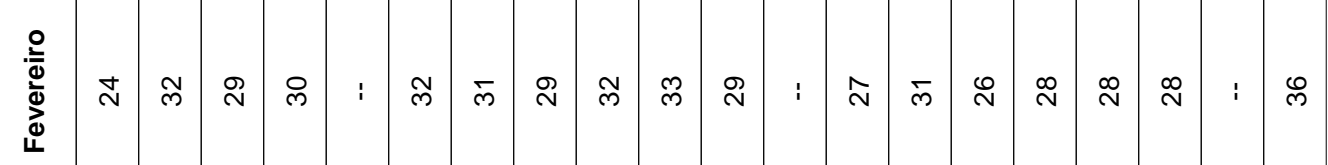

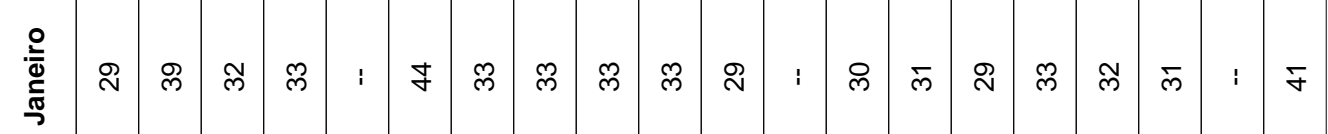

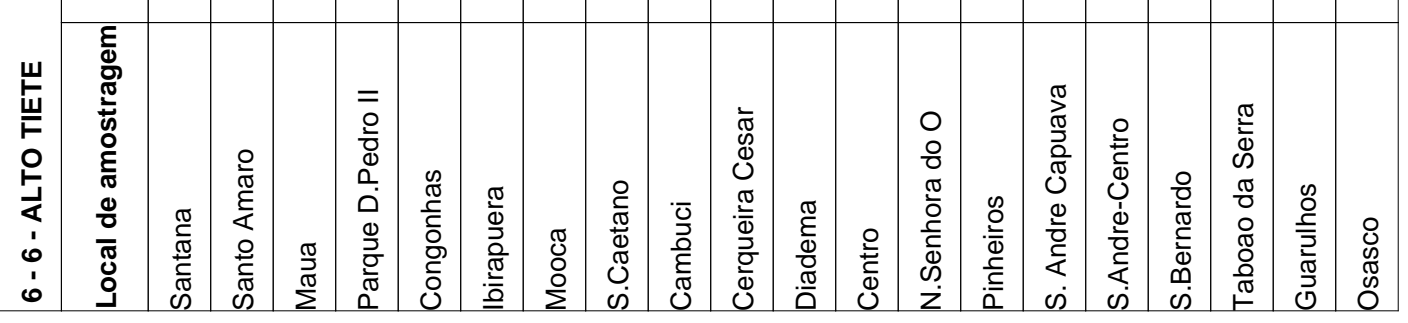




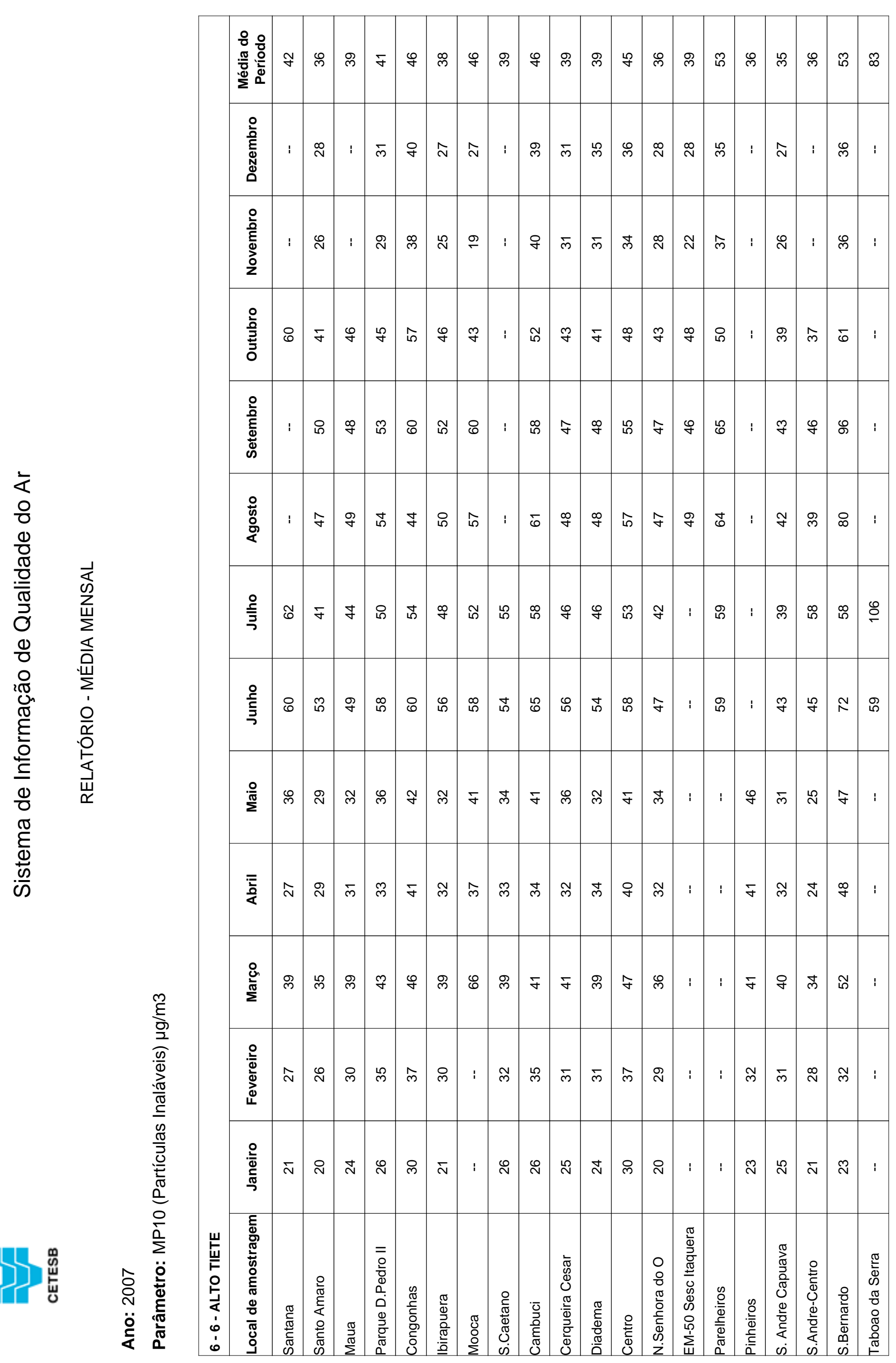




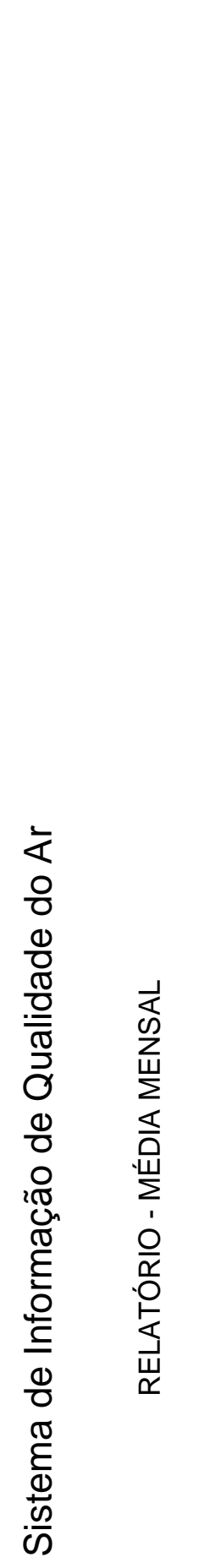

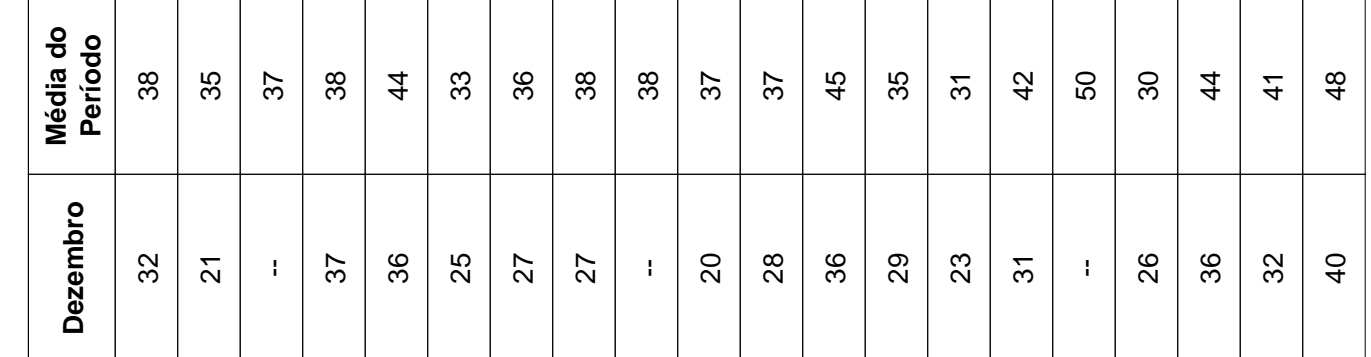

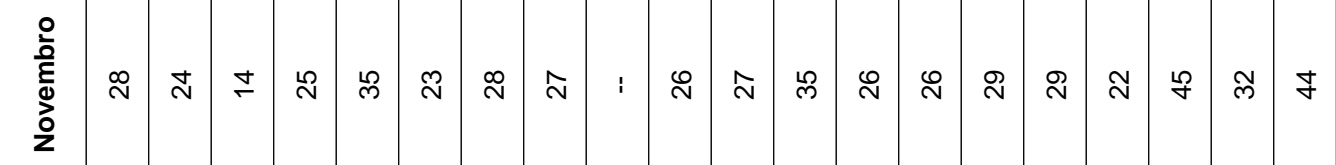

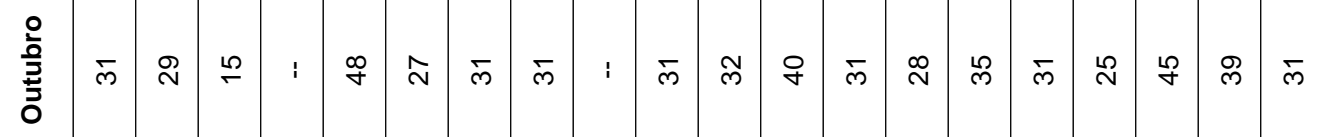

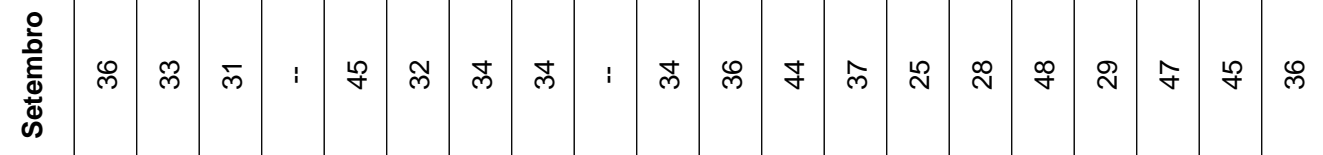

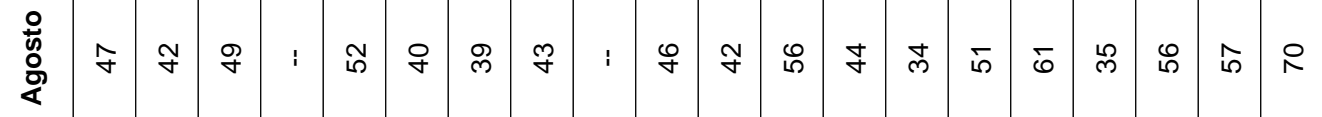

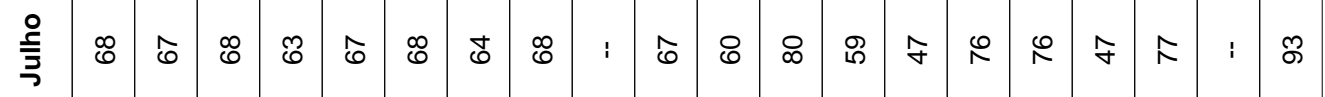

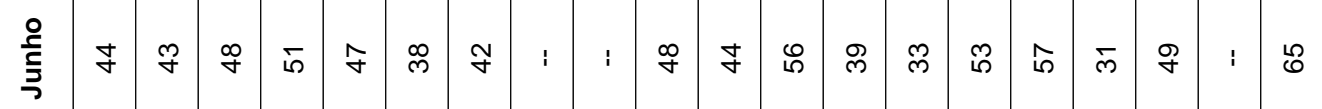

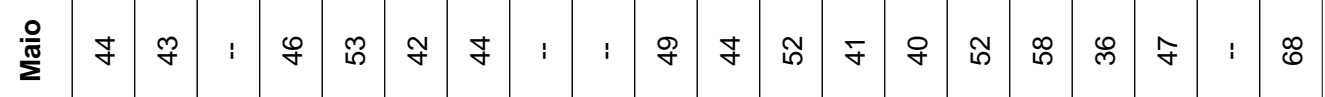

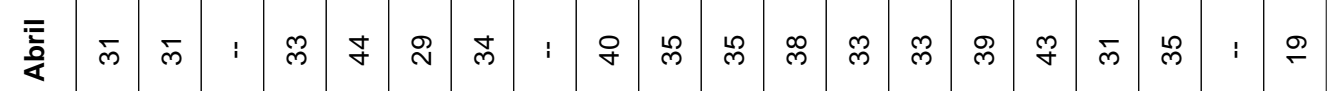

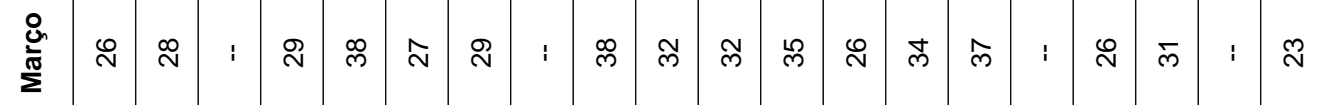

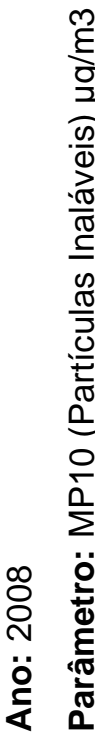

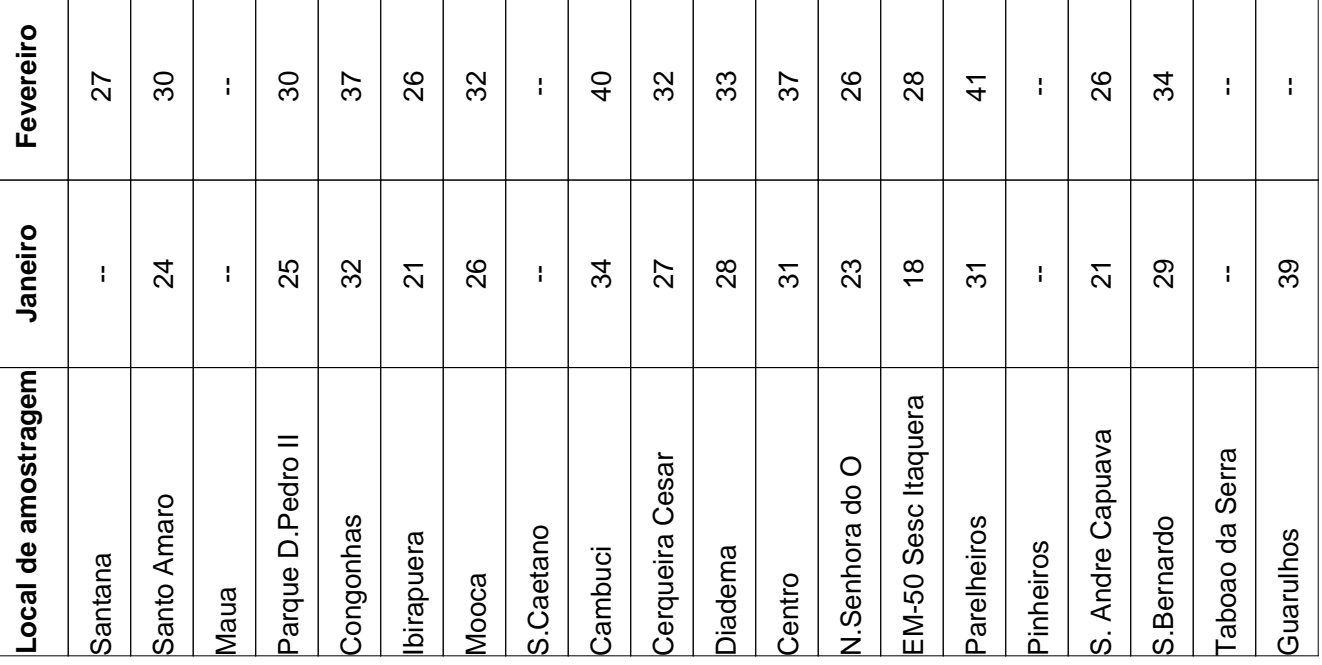




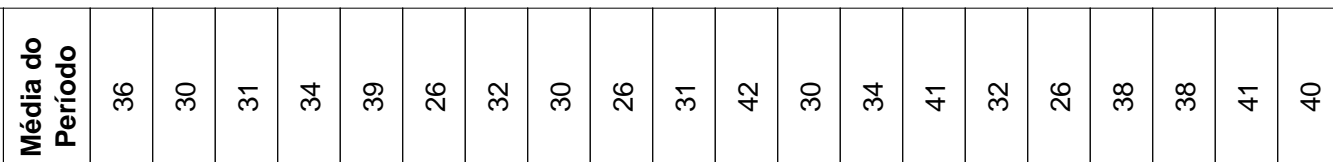

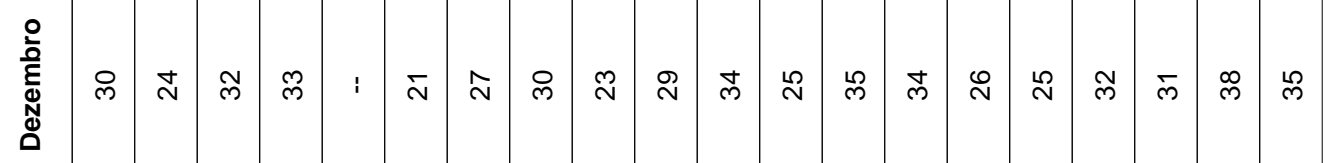

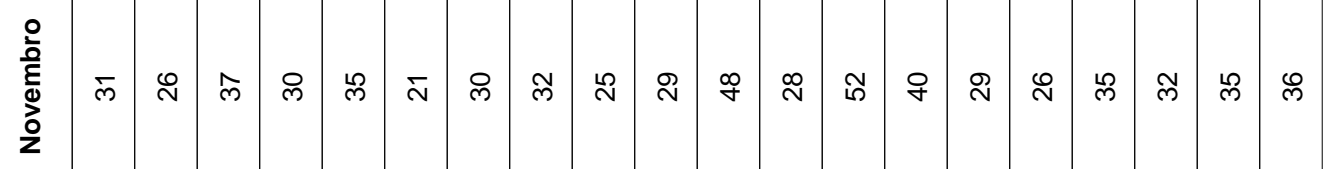

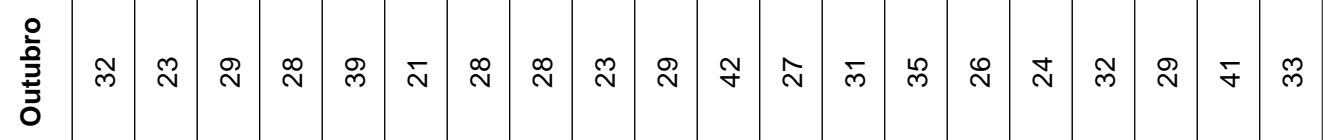

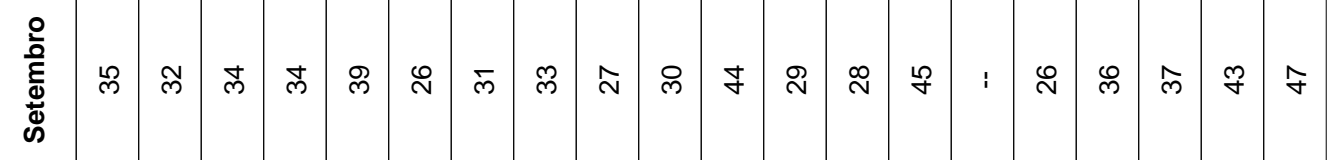

$$
\begin{aligned}
& \text { 离 }
\end{aligned}
$$

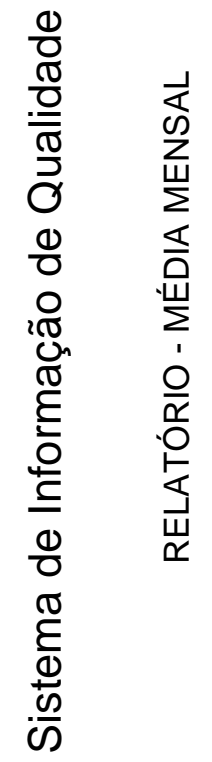$$
\text { 煞 }
$$$$
\text { 亮 }
$$$$
\text { 产 }
$$

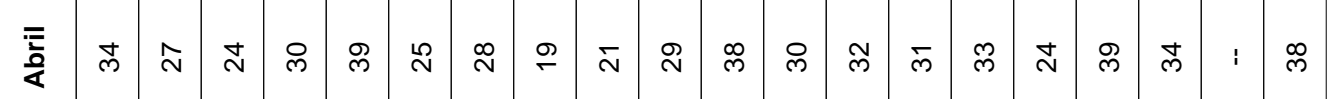

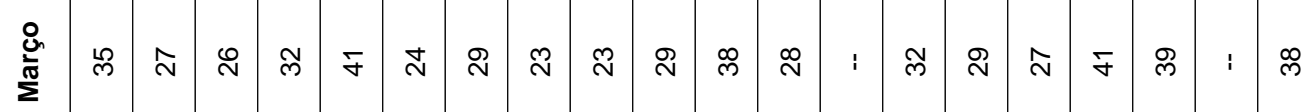

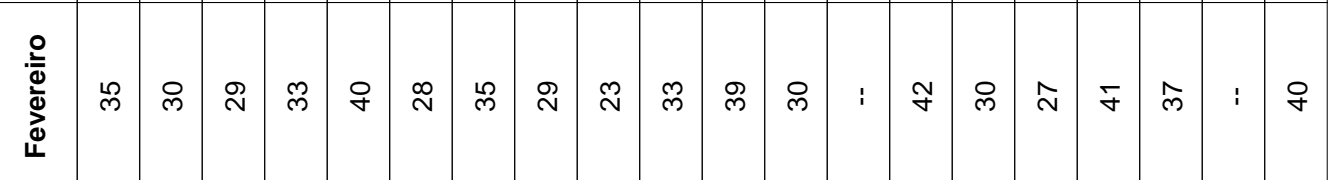

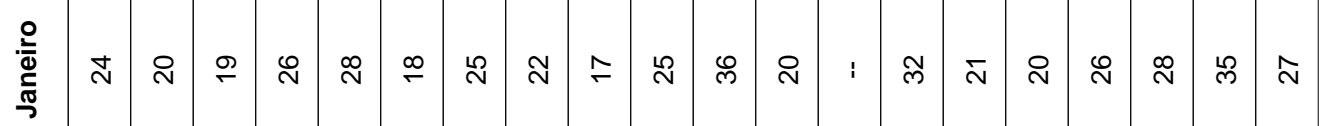

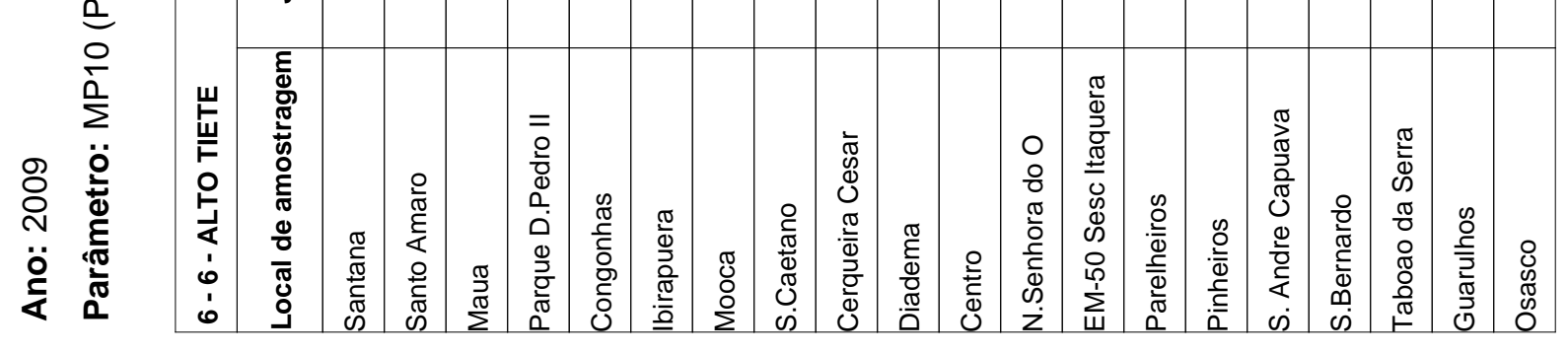




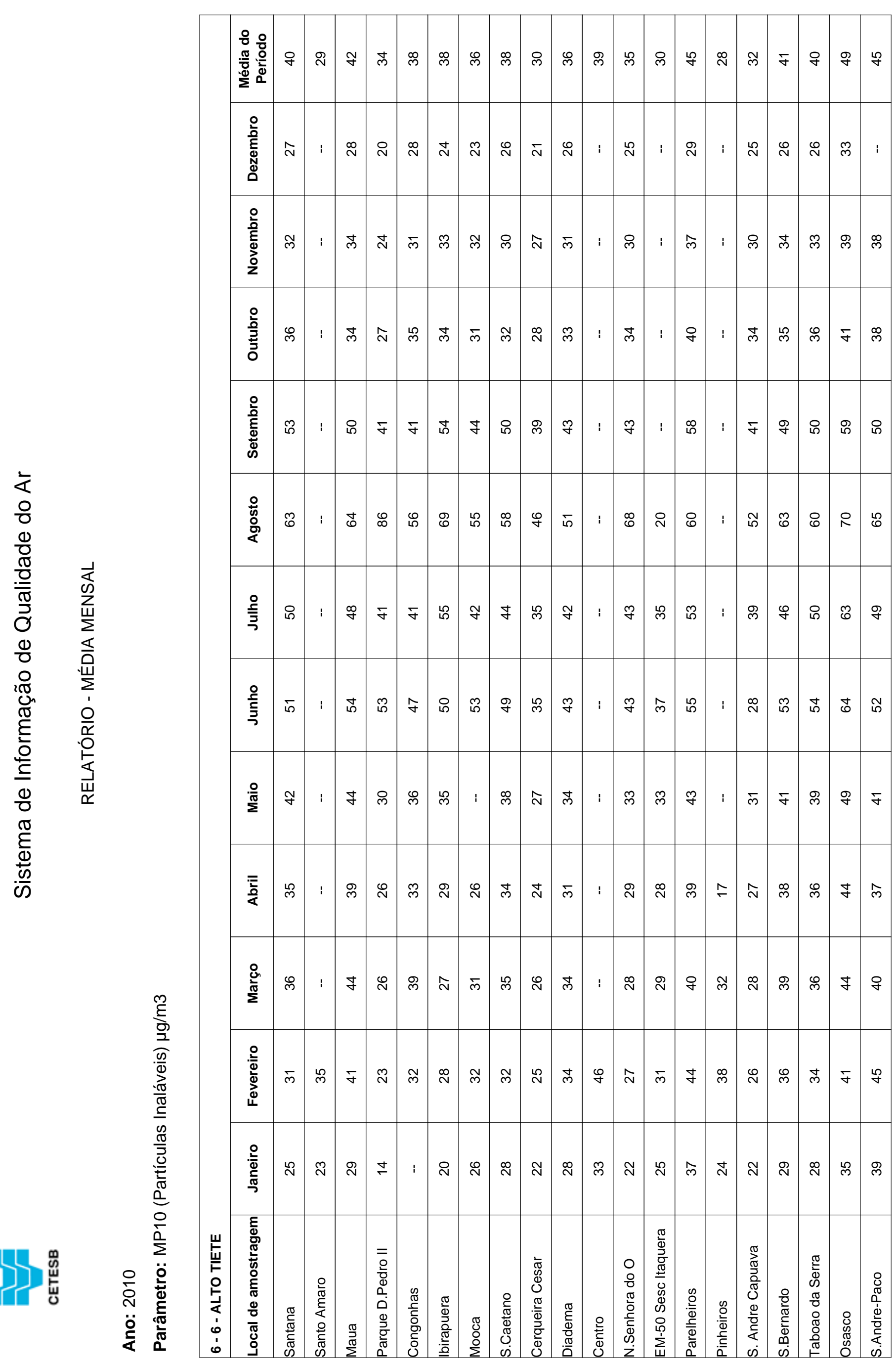




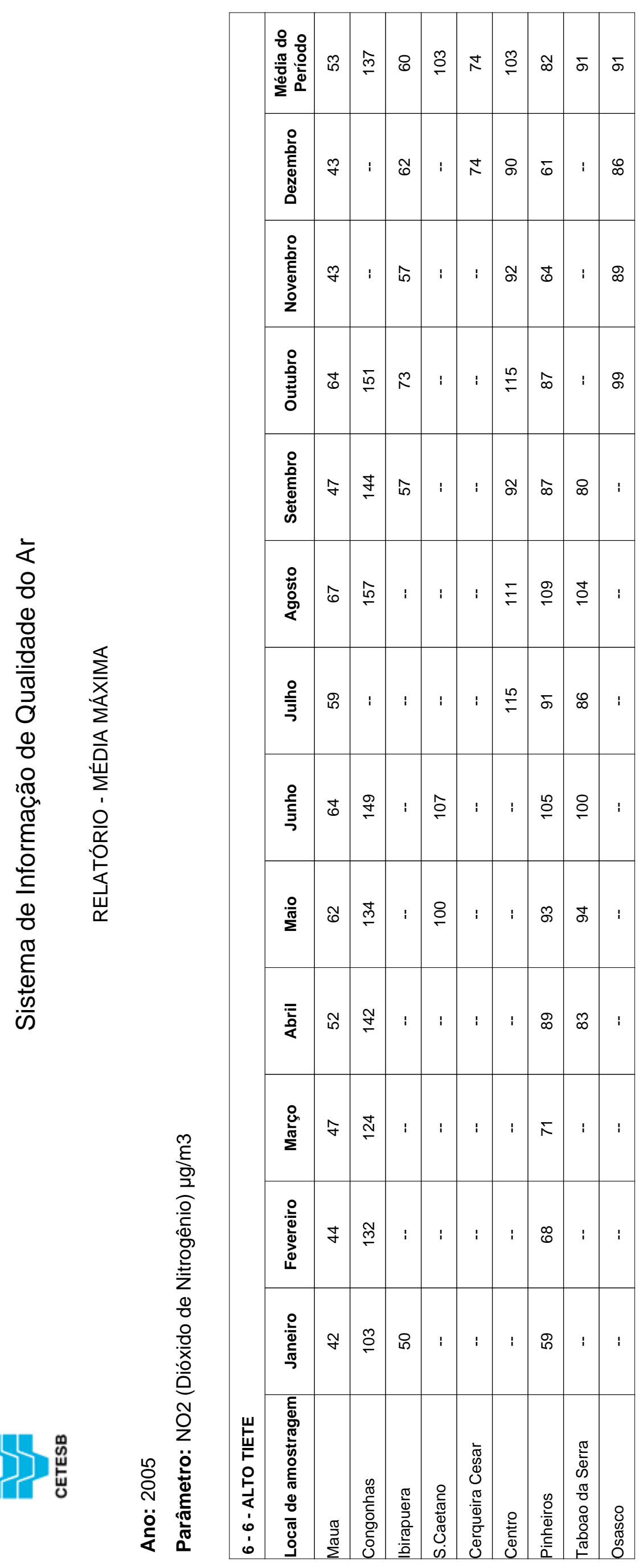




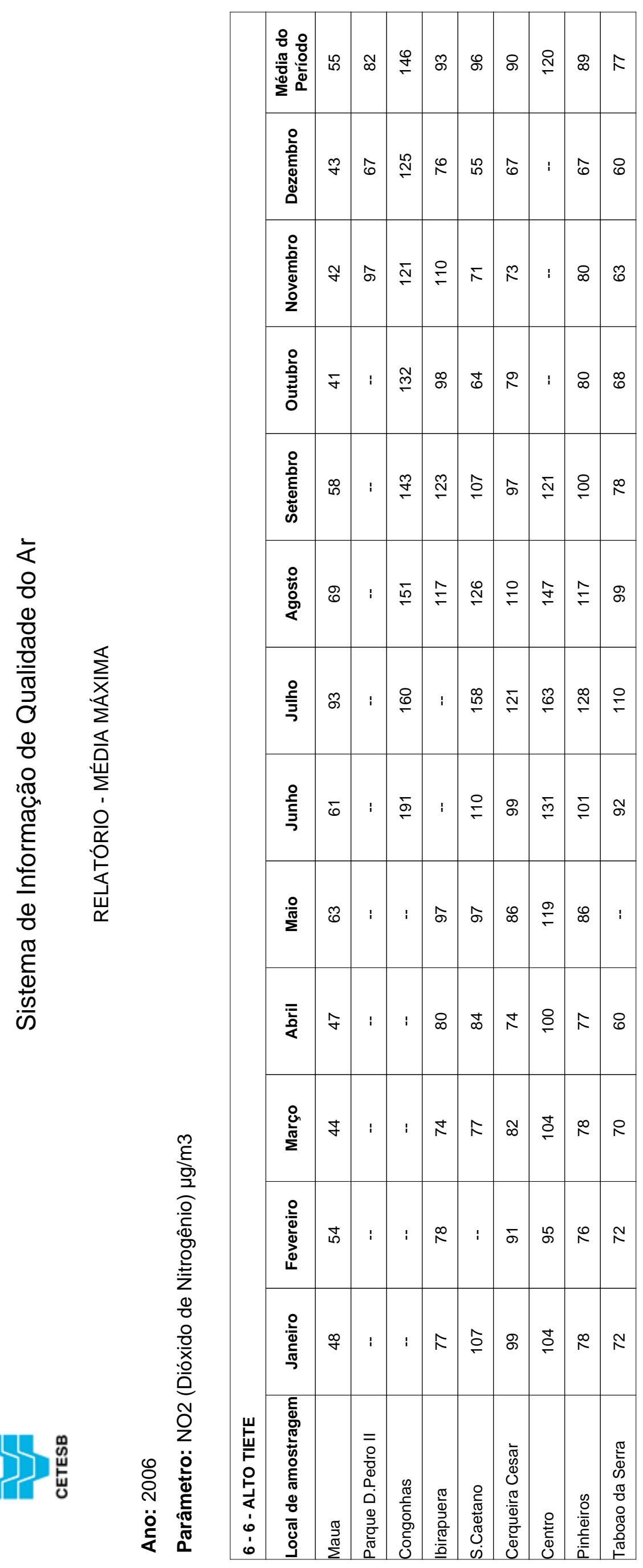




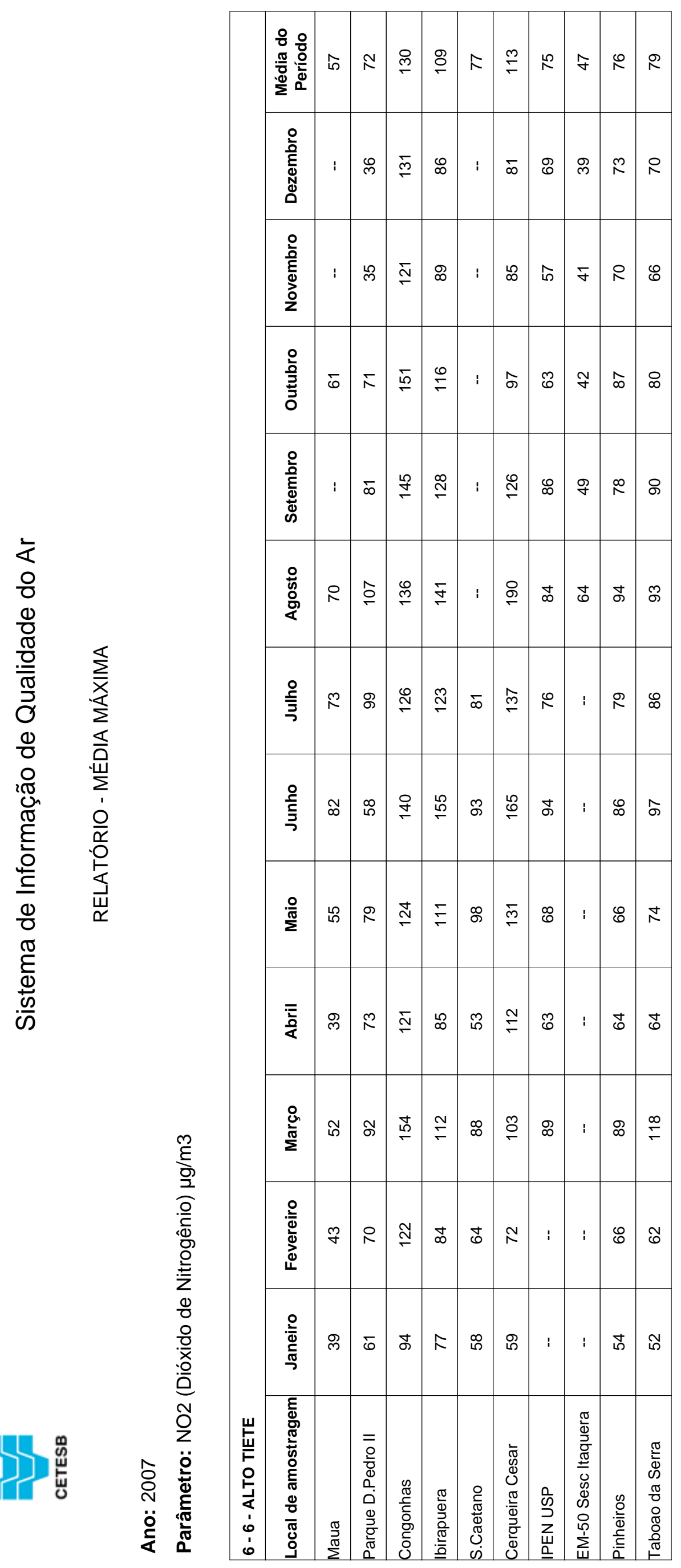




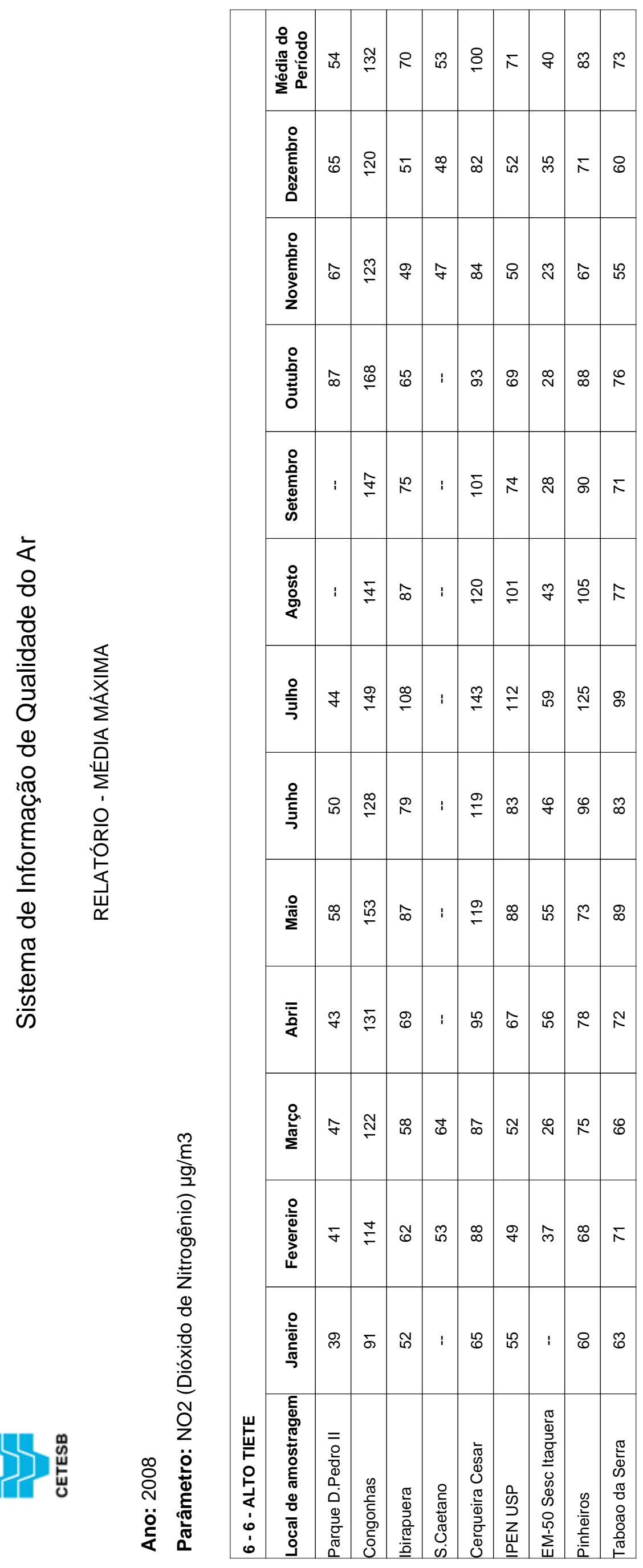




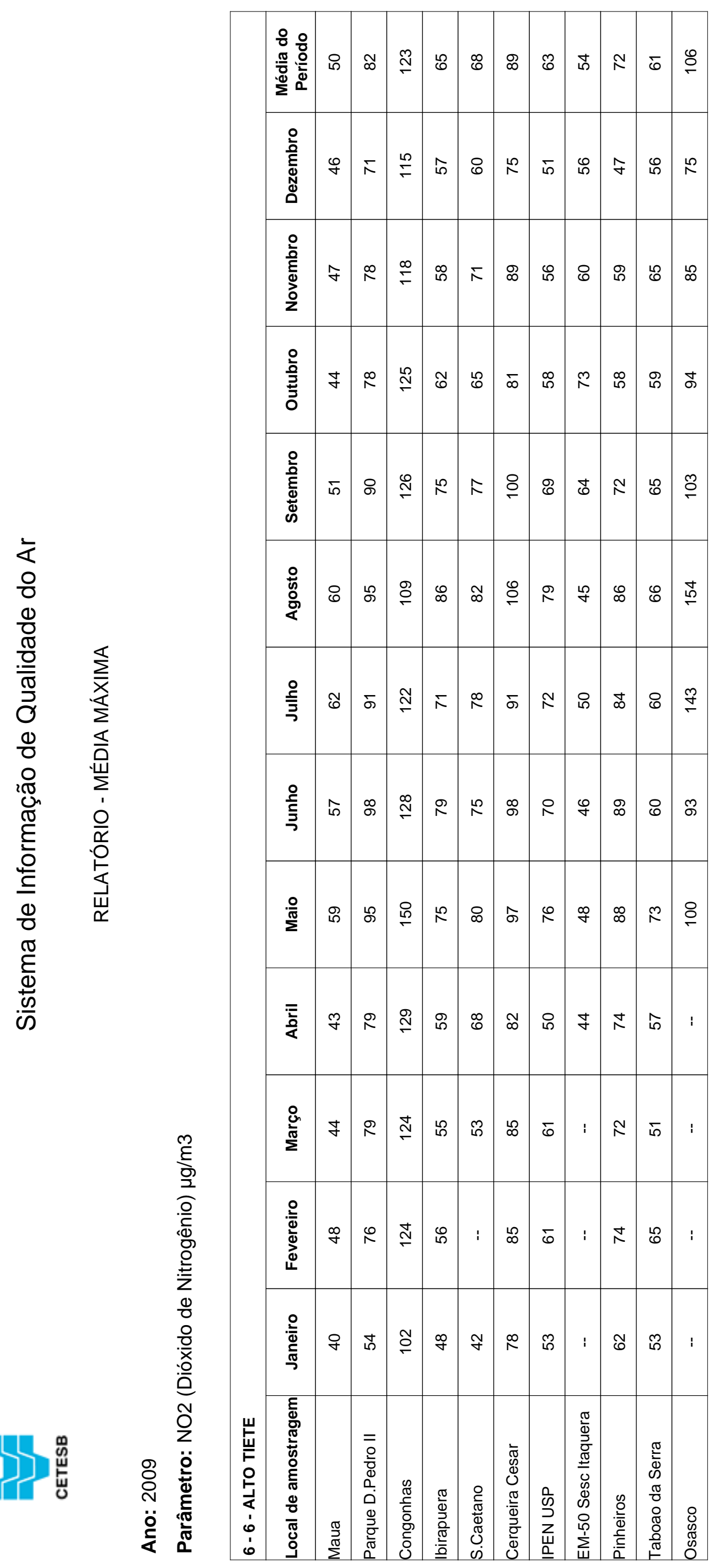




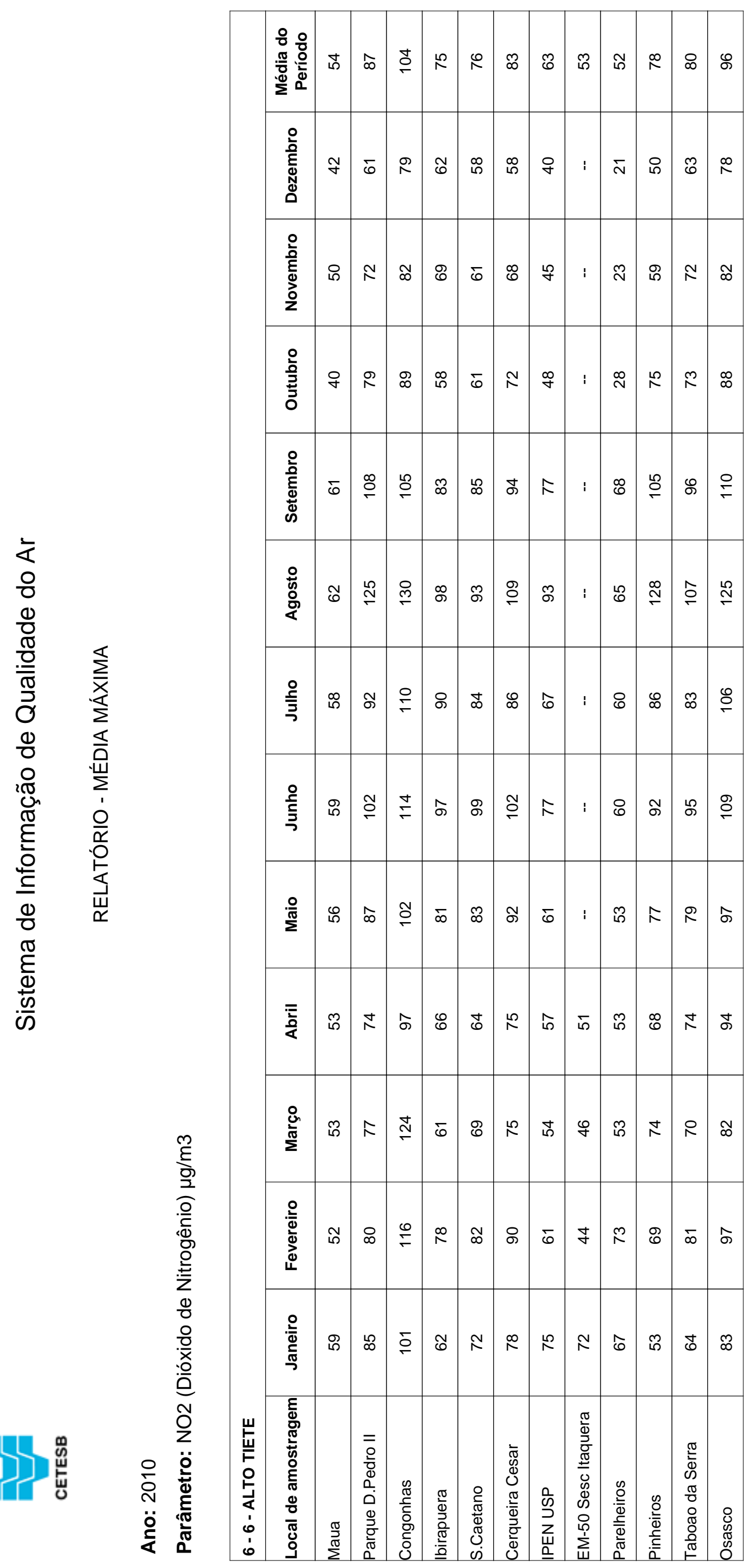




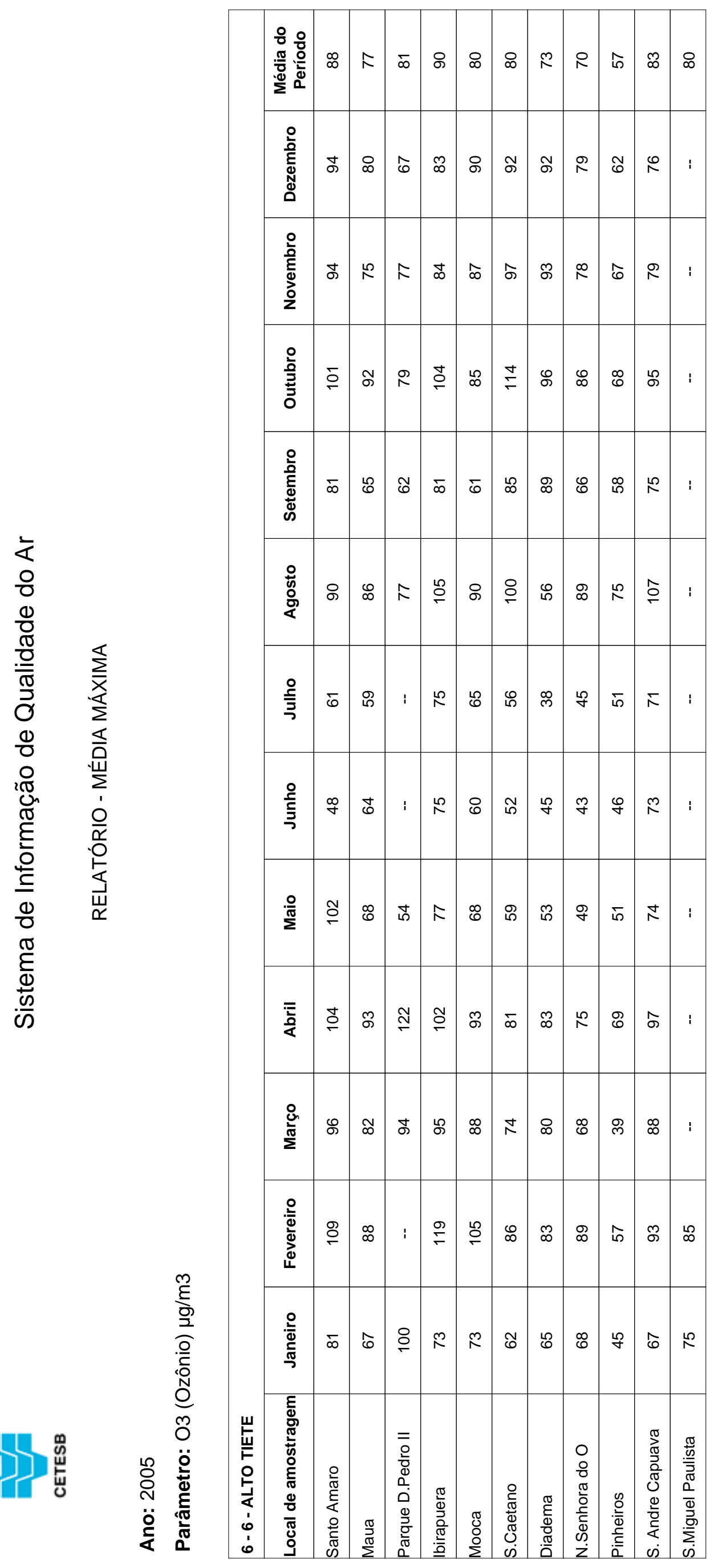




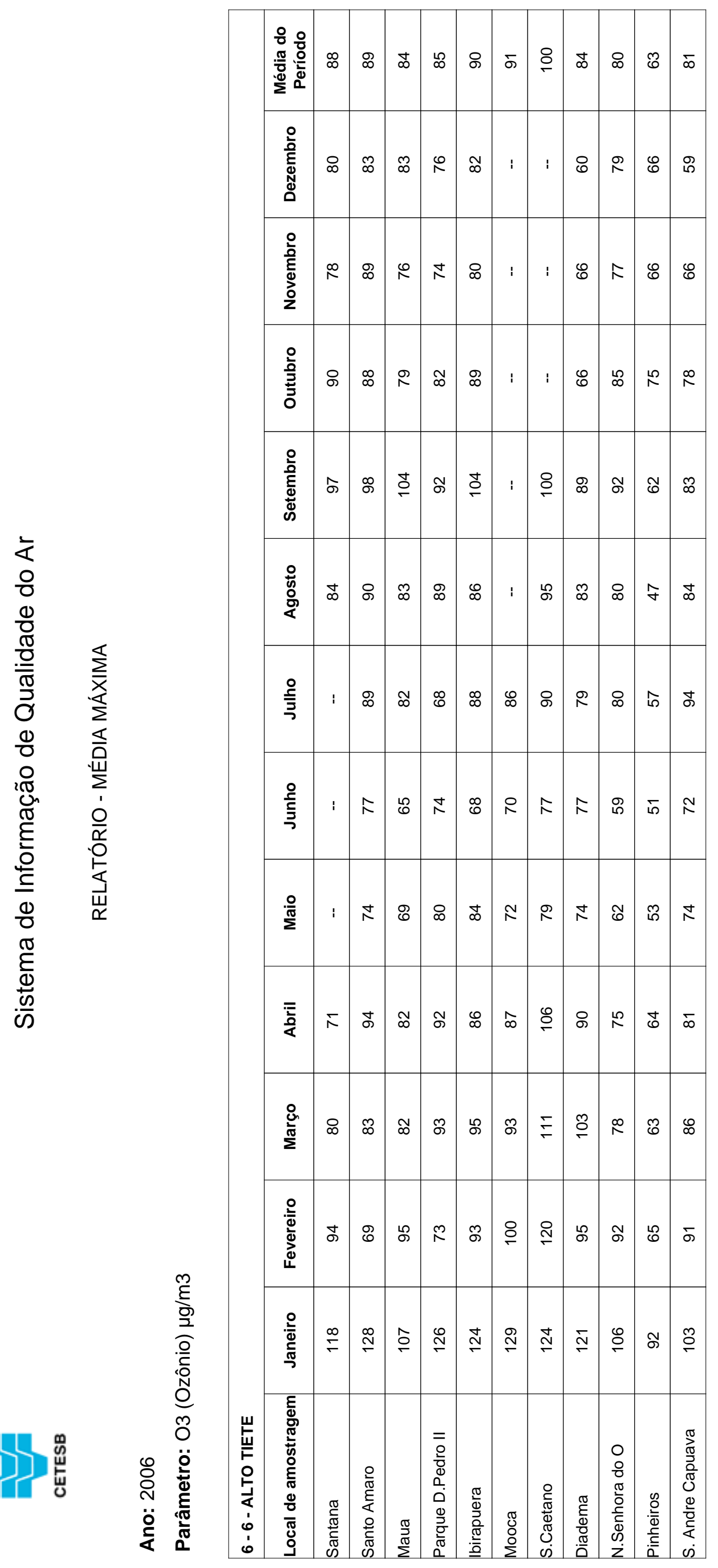




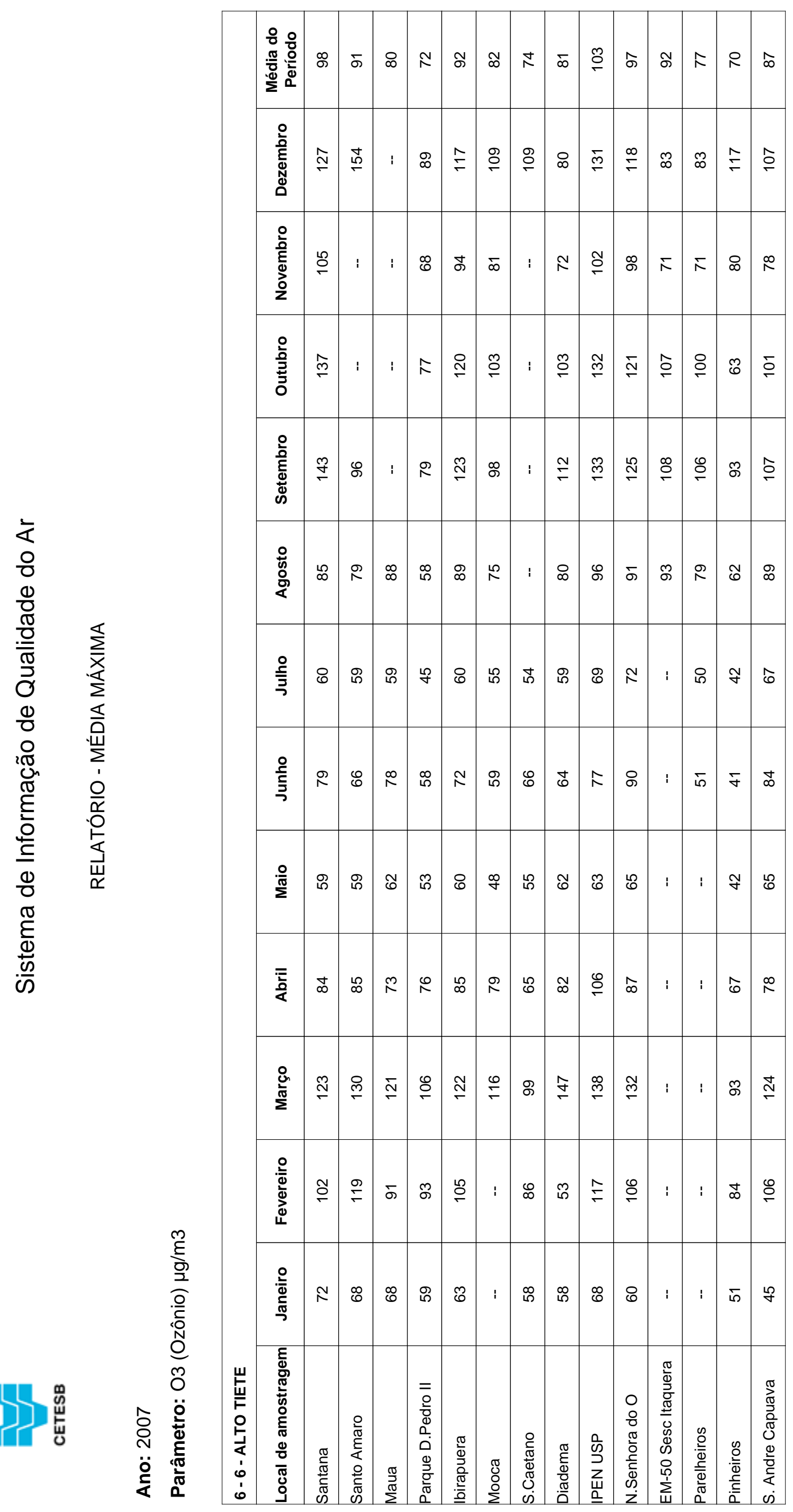




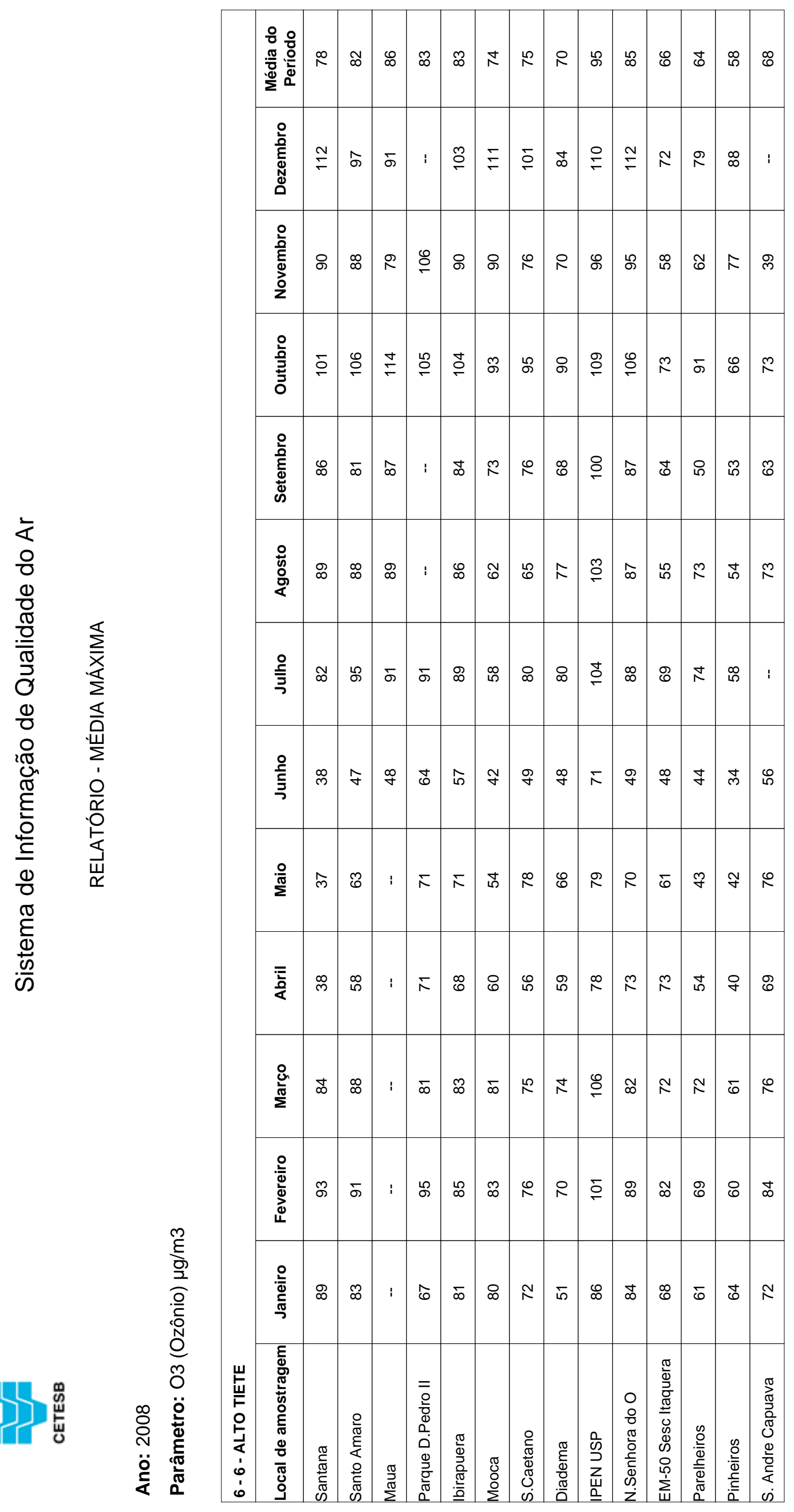




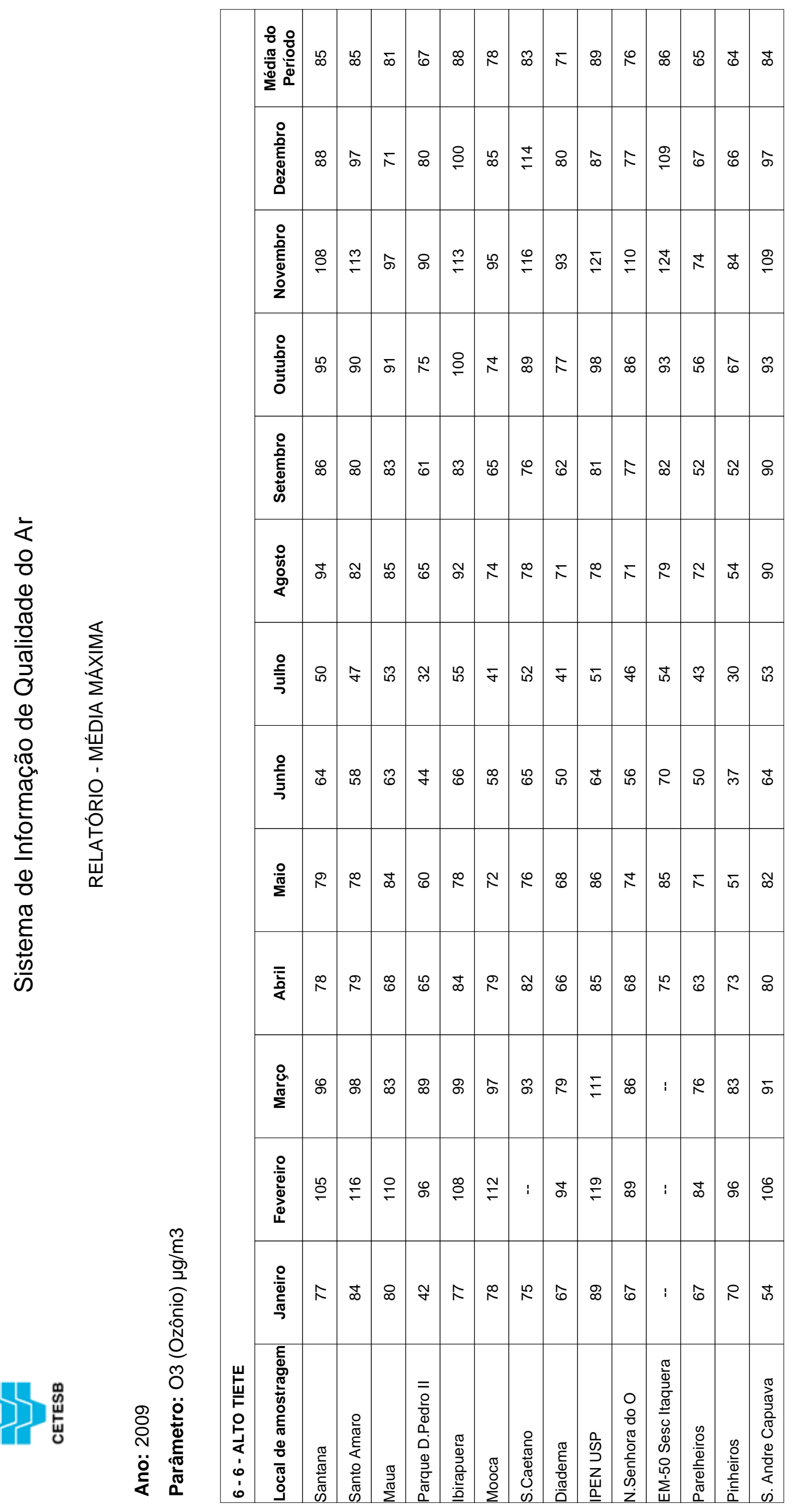




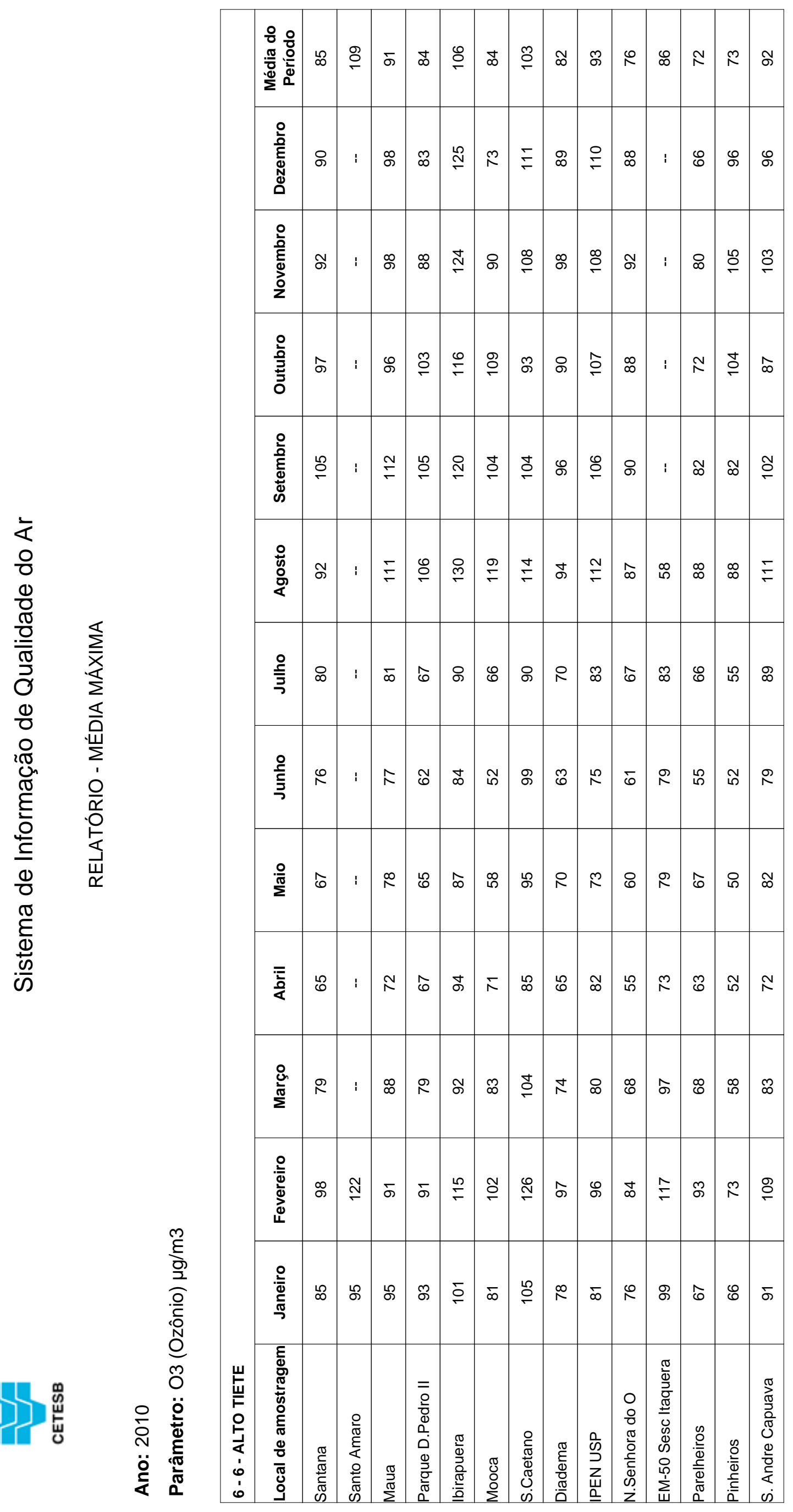




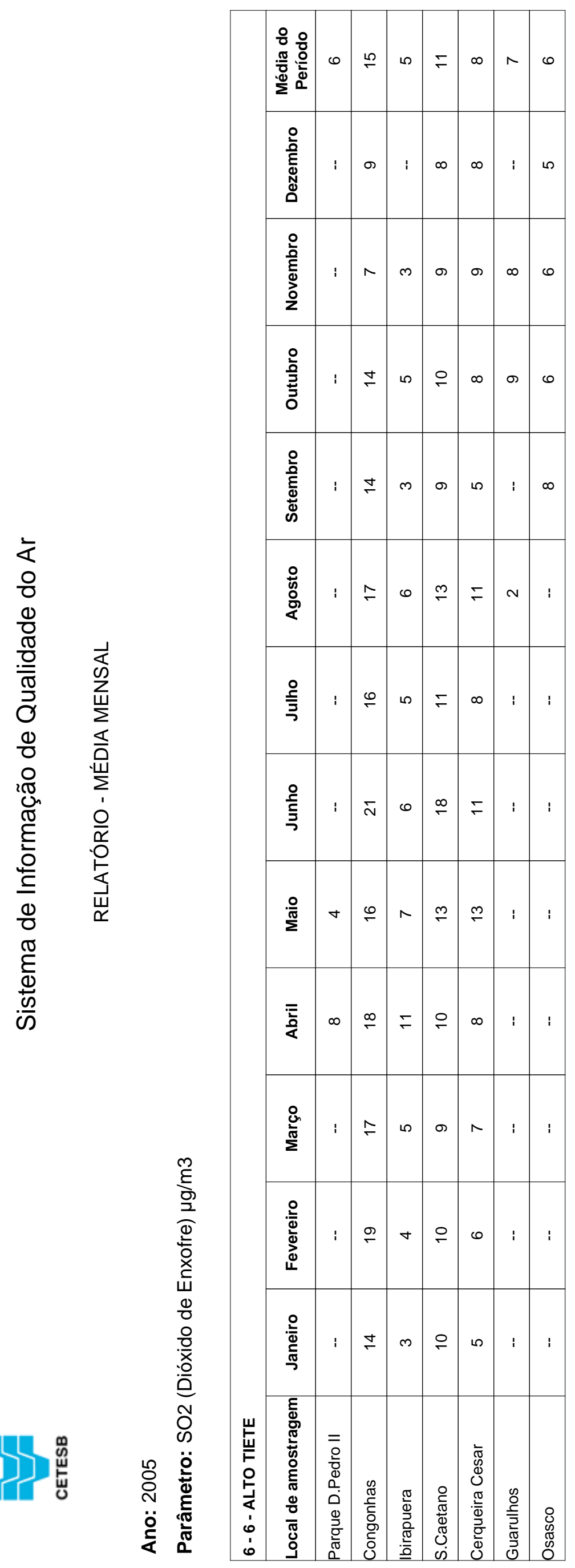




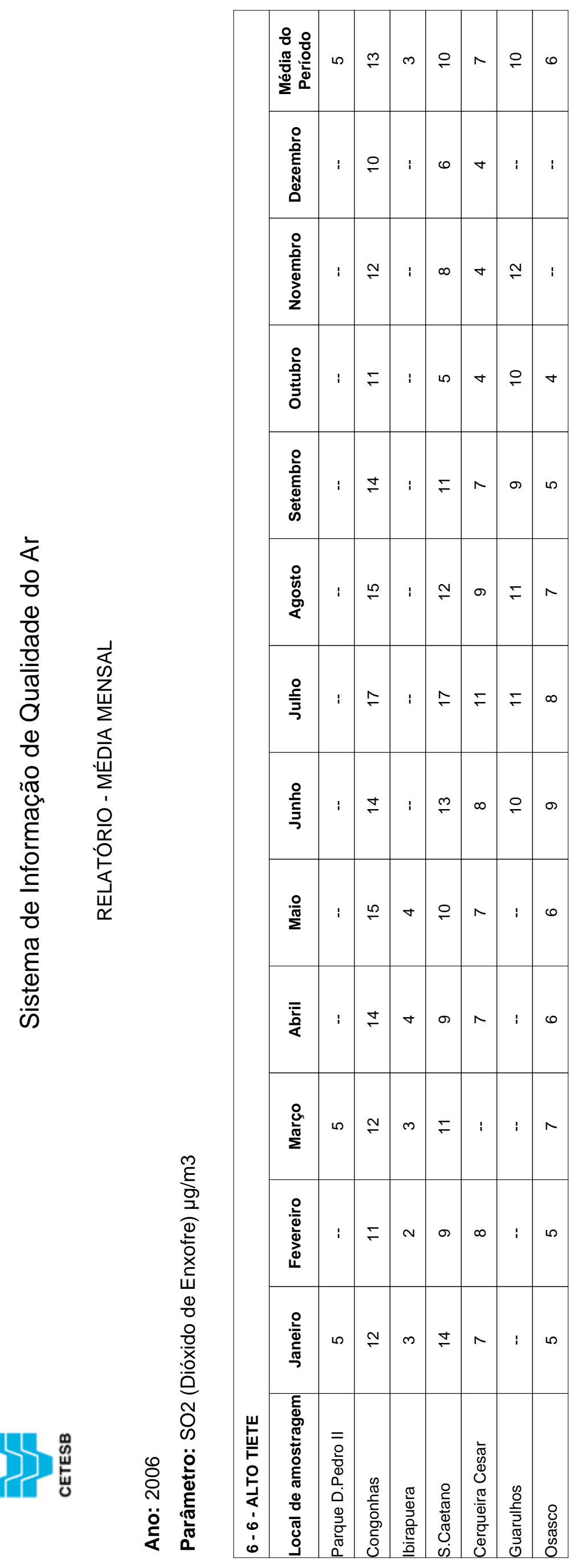




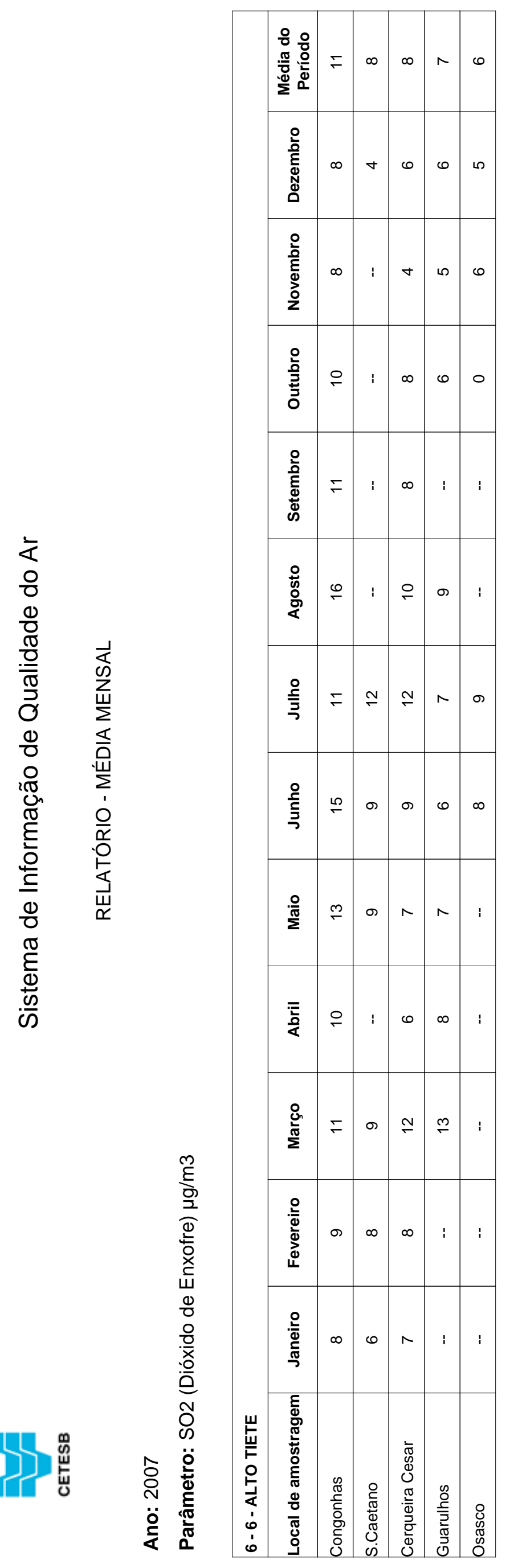




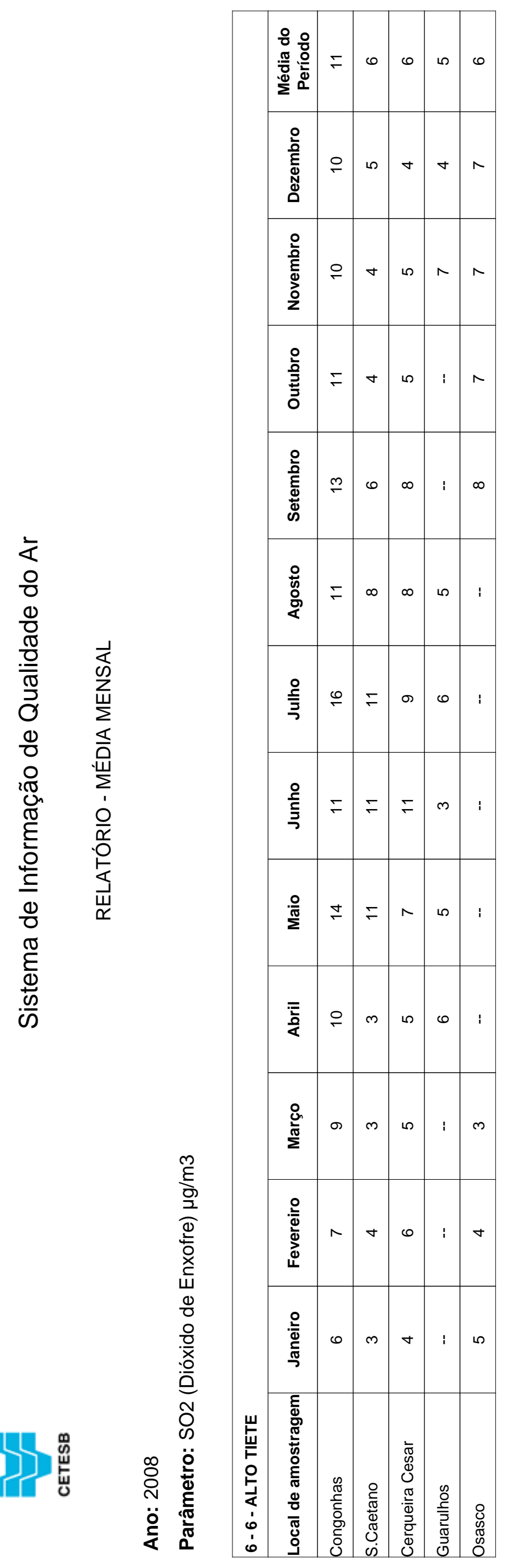




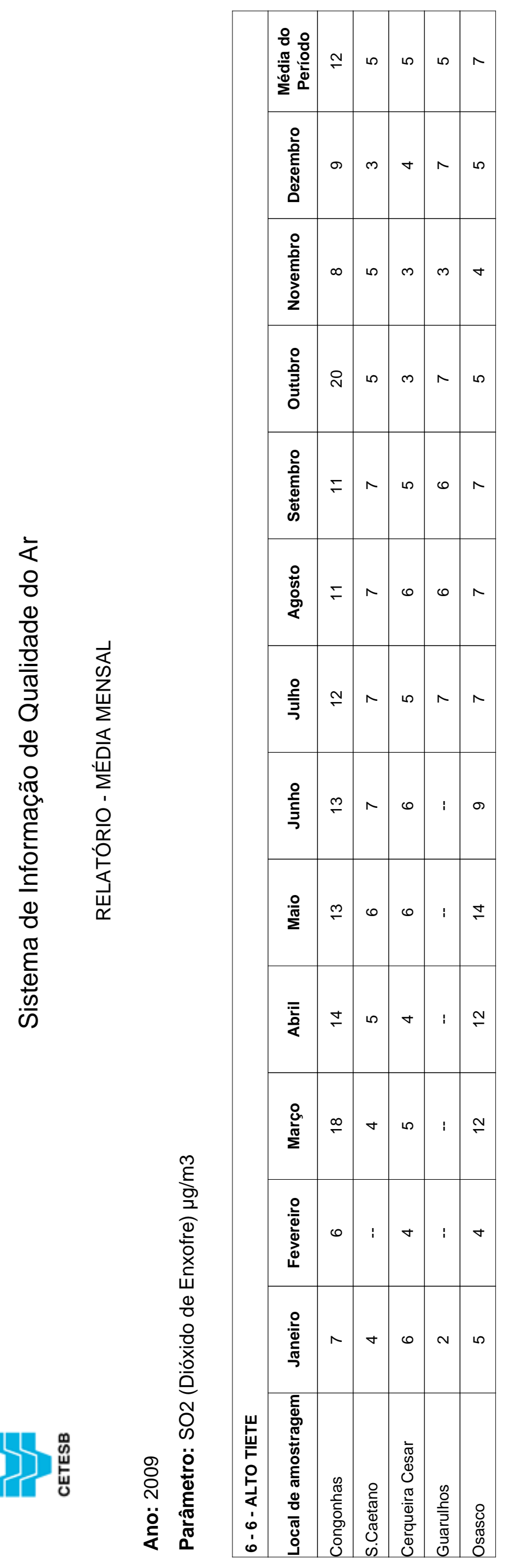




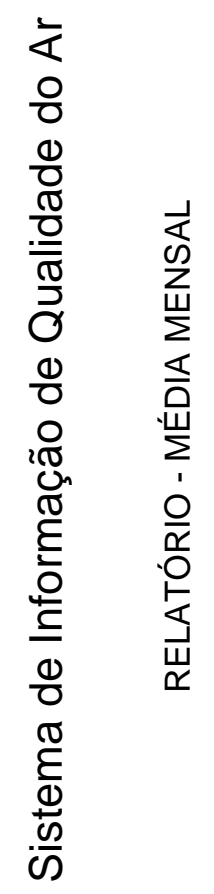

\begin{tabular}{|c|c|c|c|c|c|c|}
\hline & & 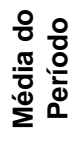 & $\infty$ & م) & $\forall$ & 0 \\
\hline & & 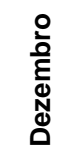 & 6 & $\theta$ & $\sim$ & $\forall$ \\
\hline & & 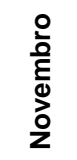 & $\wedge$ & 0 & $\nabla$ & 0 \\
\hline & & $\begin{array}{l}\text { 옹 } \\
\text { 을 } \\
\text { 호 }\end{array}$ & $\omega$ & 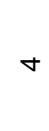 & m & 0 \\
\hline & & $\begin{array}{l}\text { 을 } \\
\text { है } \\
\text { Фँ } \\
\text { ले }\end{array}$ & $\infty$ & 0 & م & 0 \\
\hline & & $\begin{array}{l}\frac{0}{0} \\
\text { के } \\
\text { Q }\end{array}$ & $\mp$ & 0 & م & $\Lambda$ \\
\hline & & $\frac{\stackrel{\circ}{\bar{J}}}{\mathfrak{\zeta}}$ & $\sigma$ & $\wedge$ & م & $\infty$ \\
\hline & & $\begin{array}{l}\frac{\circ}{5} \\
\frac{\mathfrak{5}}{5}\end{array}$ & 으 & 0 & $\infty$ & $\sigma$ \\
\hline & & $\frac{0}{\frac{0}{\pi N}}$ & $\infty$ & 0 & ما & 0 \\
\hline & & Г̄ & $\infty$ & م & m & $\wedge$ \\
\hline & & 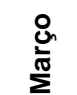 & $\mp$ & م & ما & $\infty$ \\
\hline 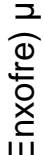 & & 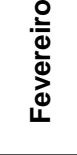 & $\infty$ & $\wedge$ & $\forall$ & 10 \\
\hline $\begin{array}{l}\frac{1}{0} \\
\frac{0}{0} \\
\frac{0}{x} \\
0 \\
0\end{array}$ & & 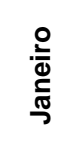 & 0 & m & m & م \\
\hline 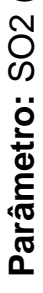 & 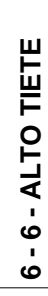 & 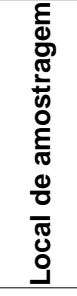 & 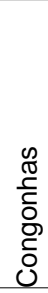 & 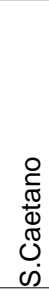 & 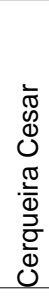 & $\begin{array}{l}\text { O } \\
\mathbb{2} \\
\mathbb{0} \\
0 \\
0\end{array}$ \\
\hline
\end{tabular}




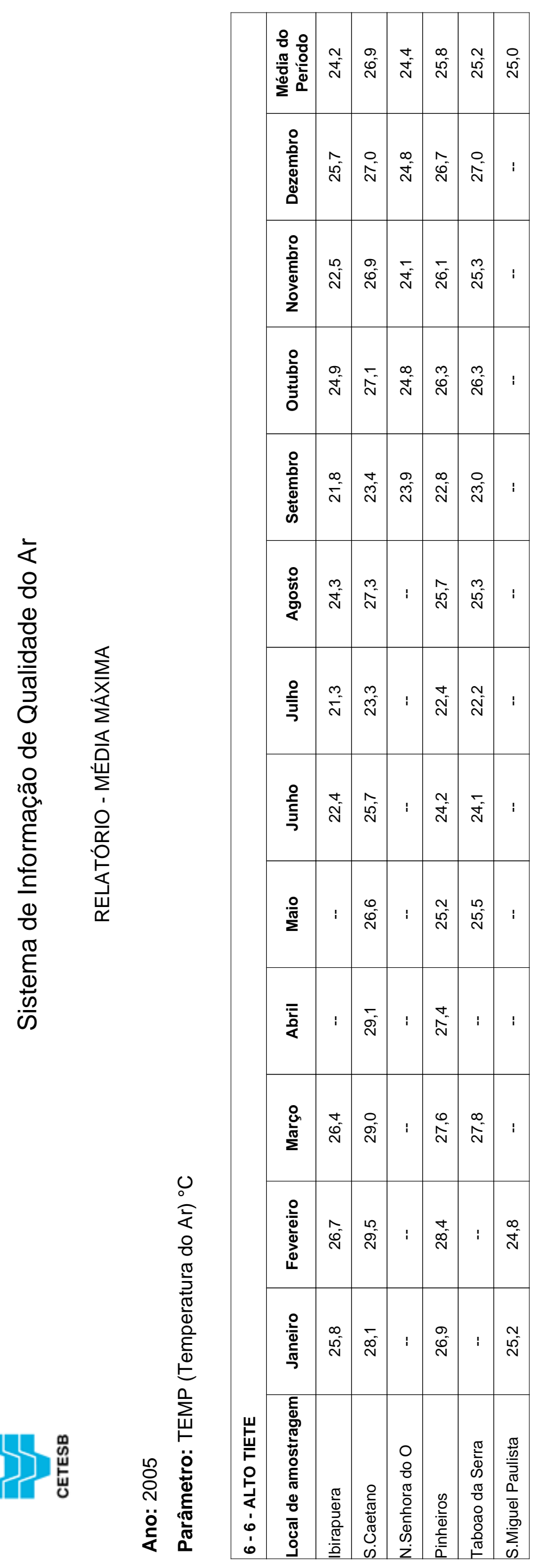




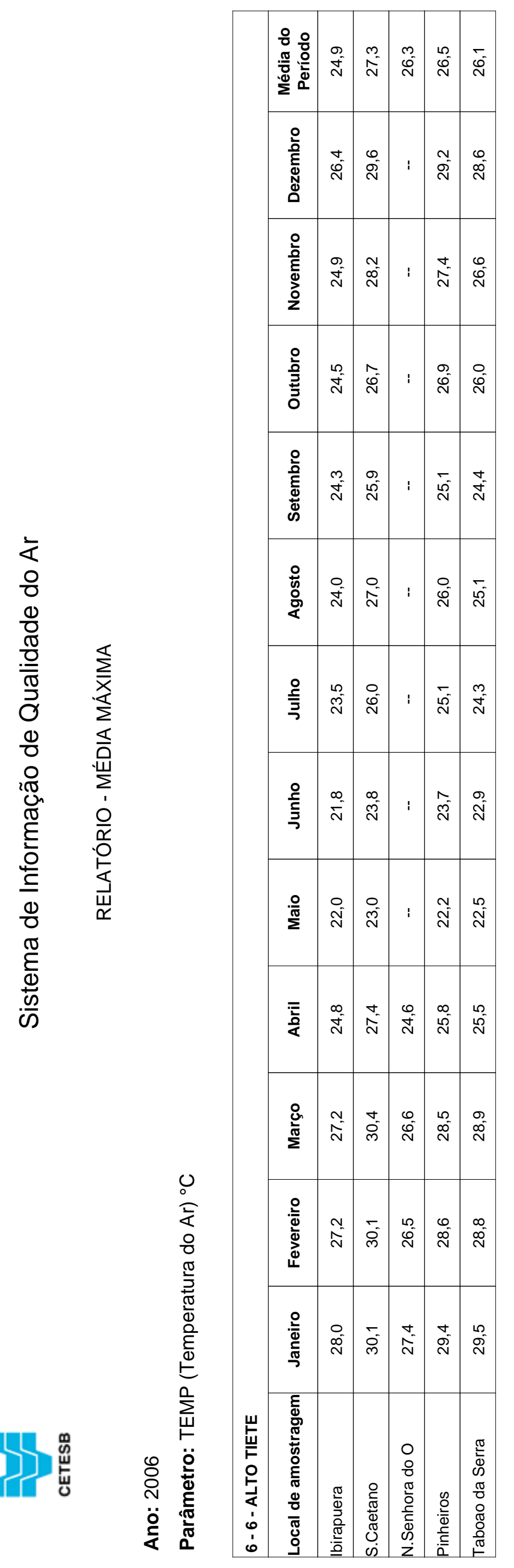




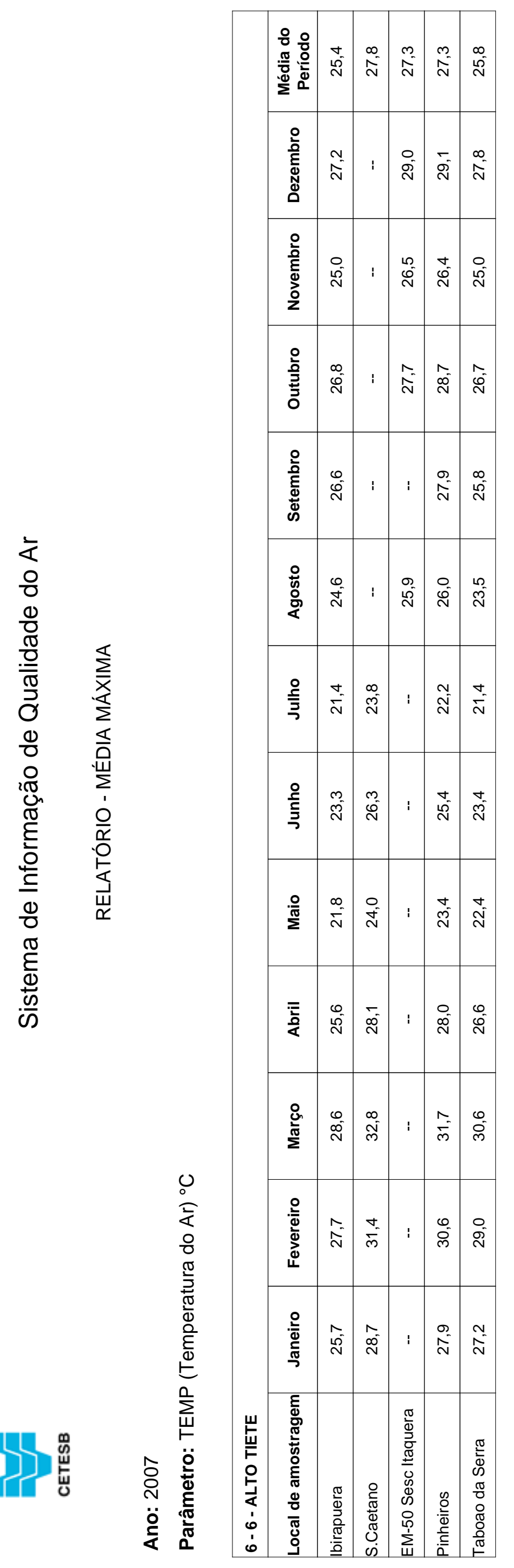




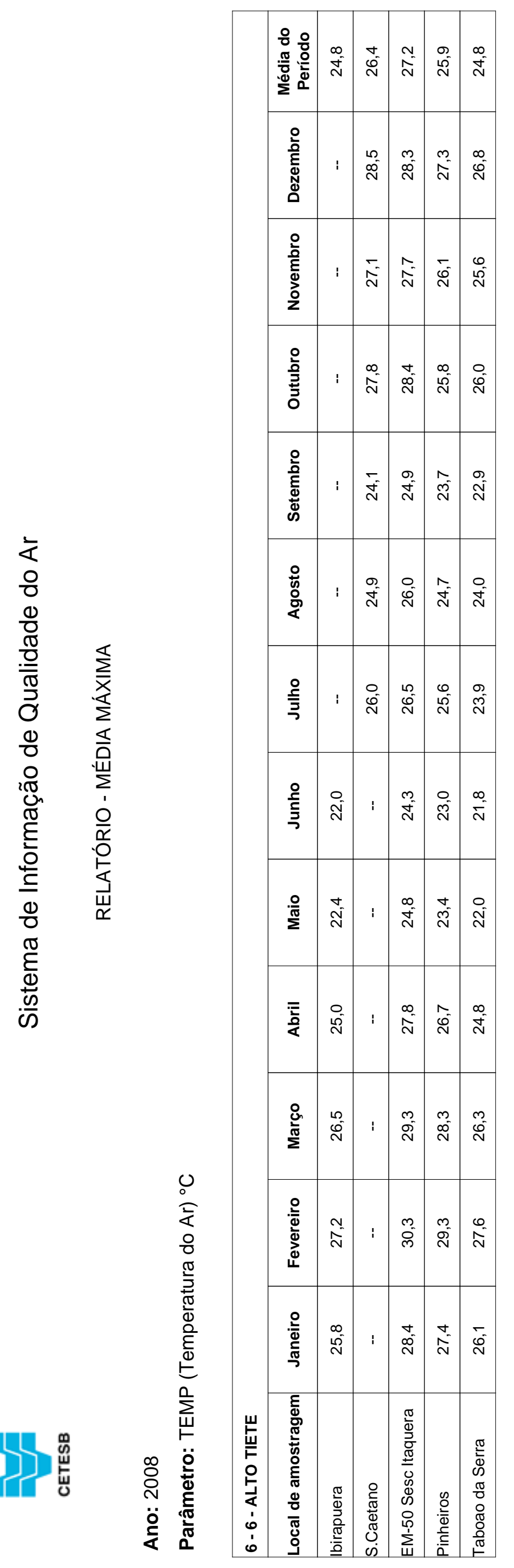




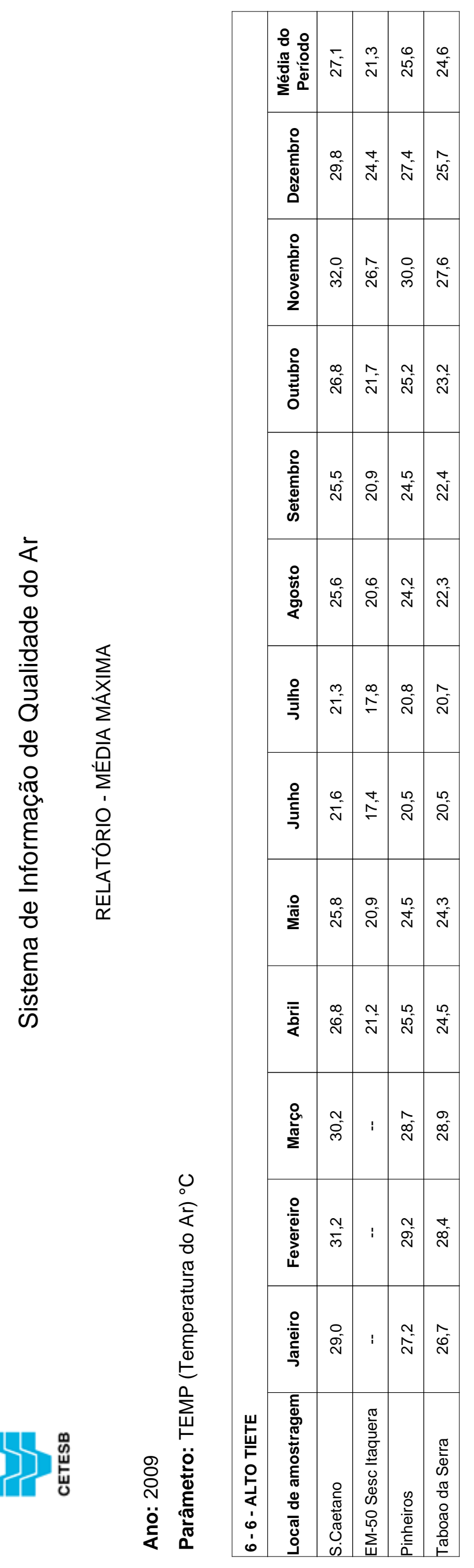




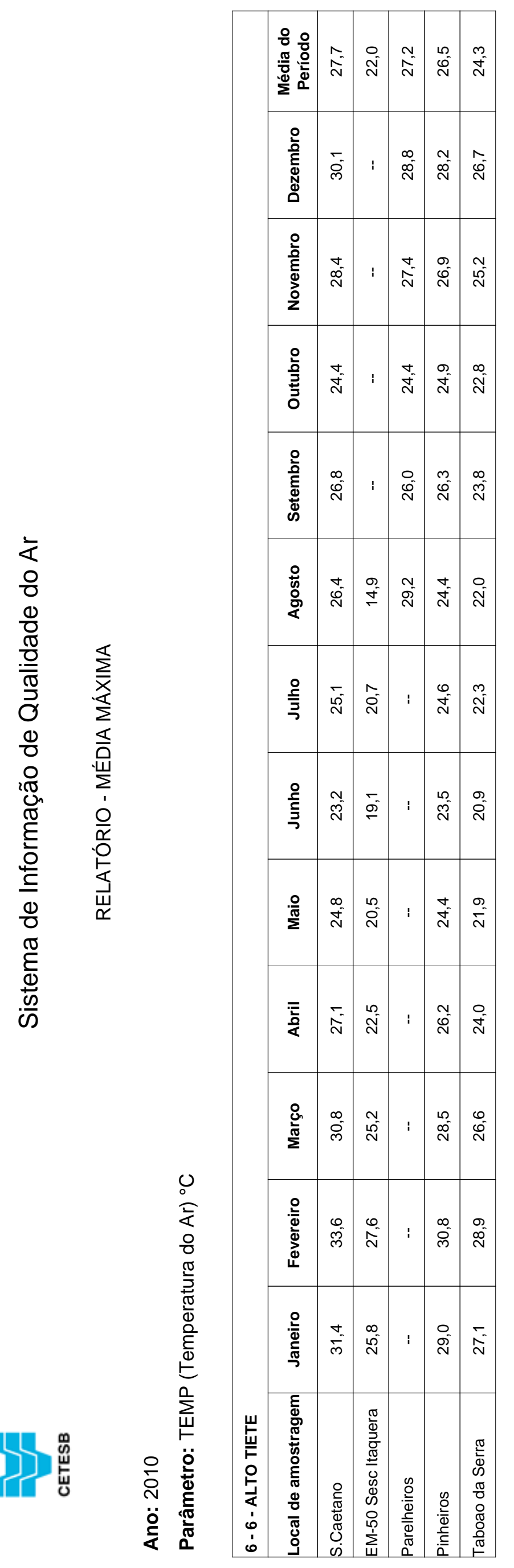




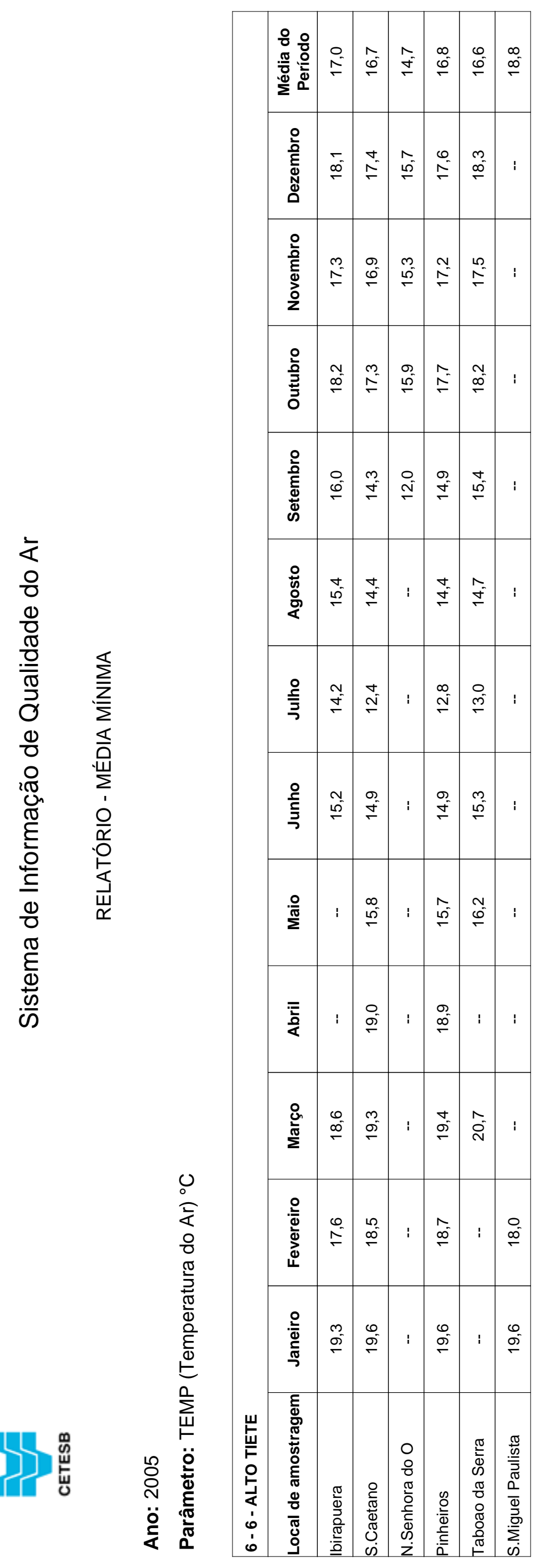




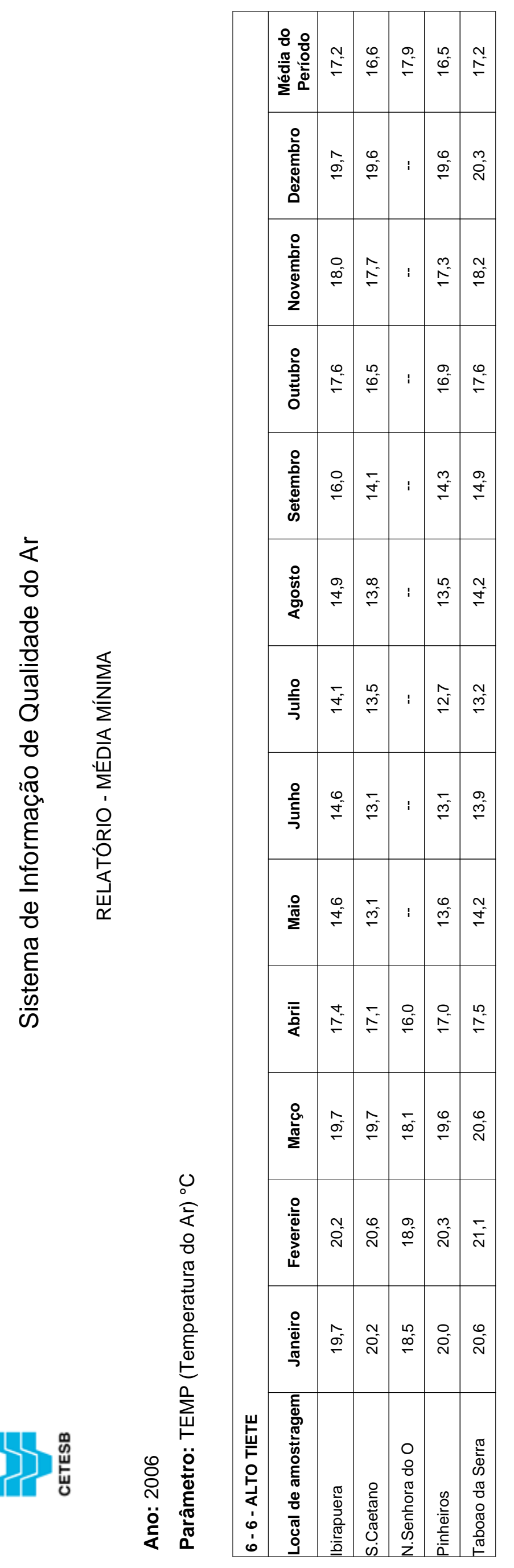




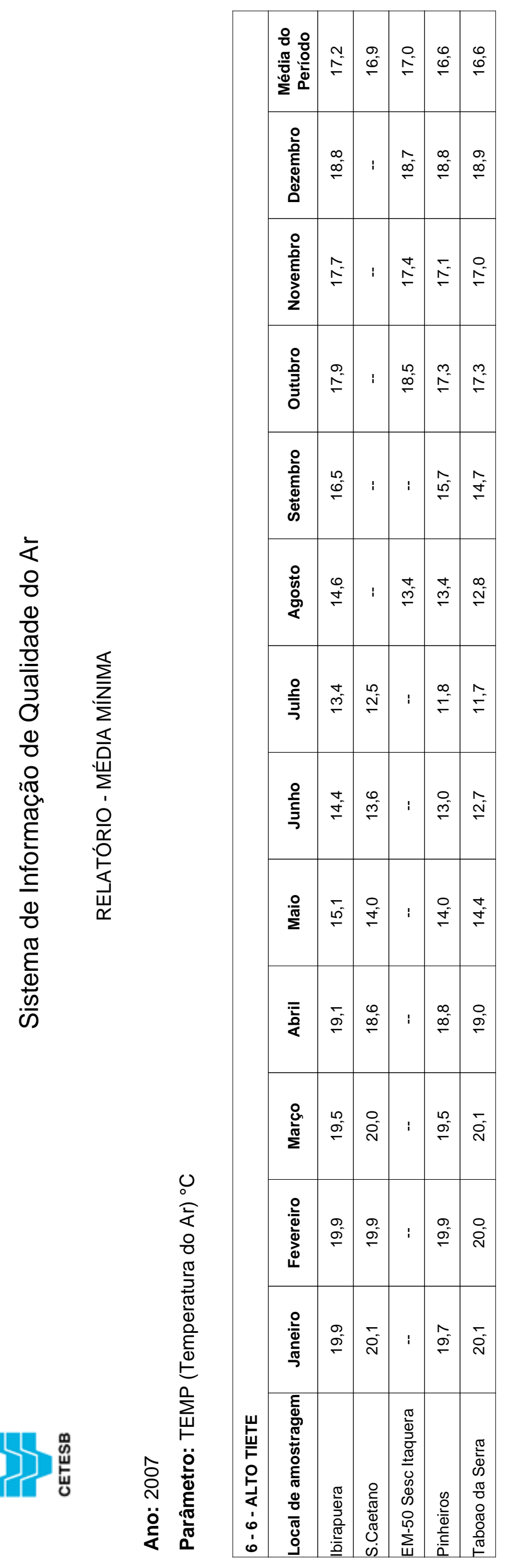




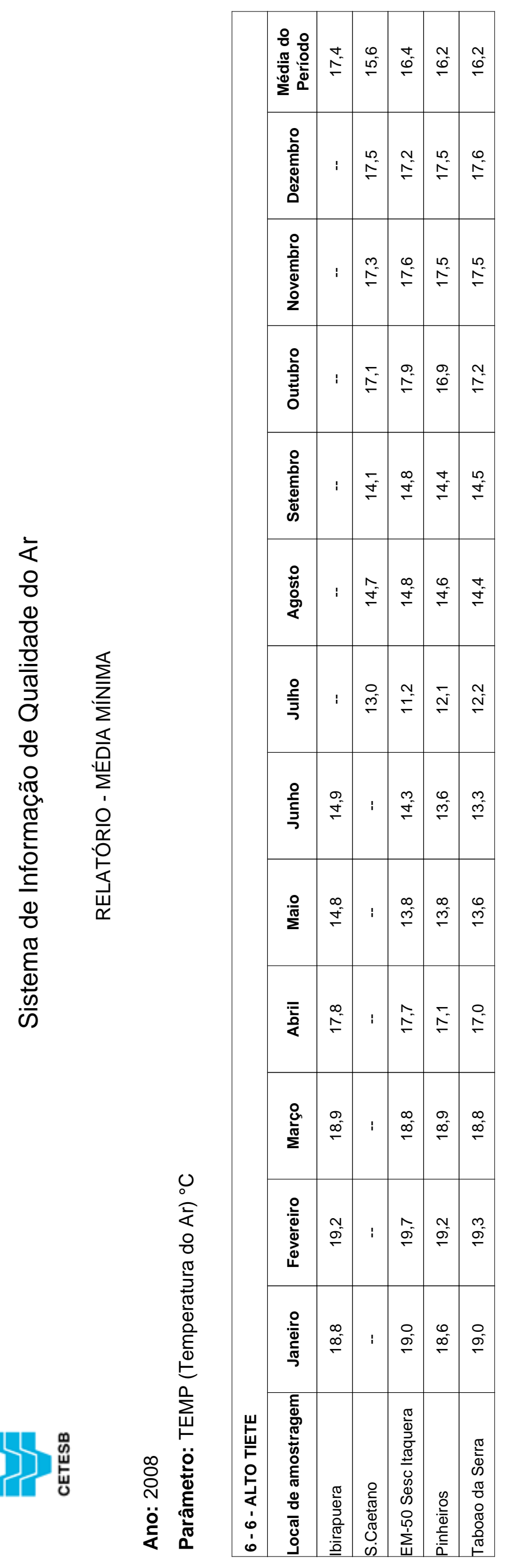




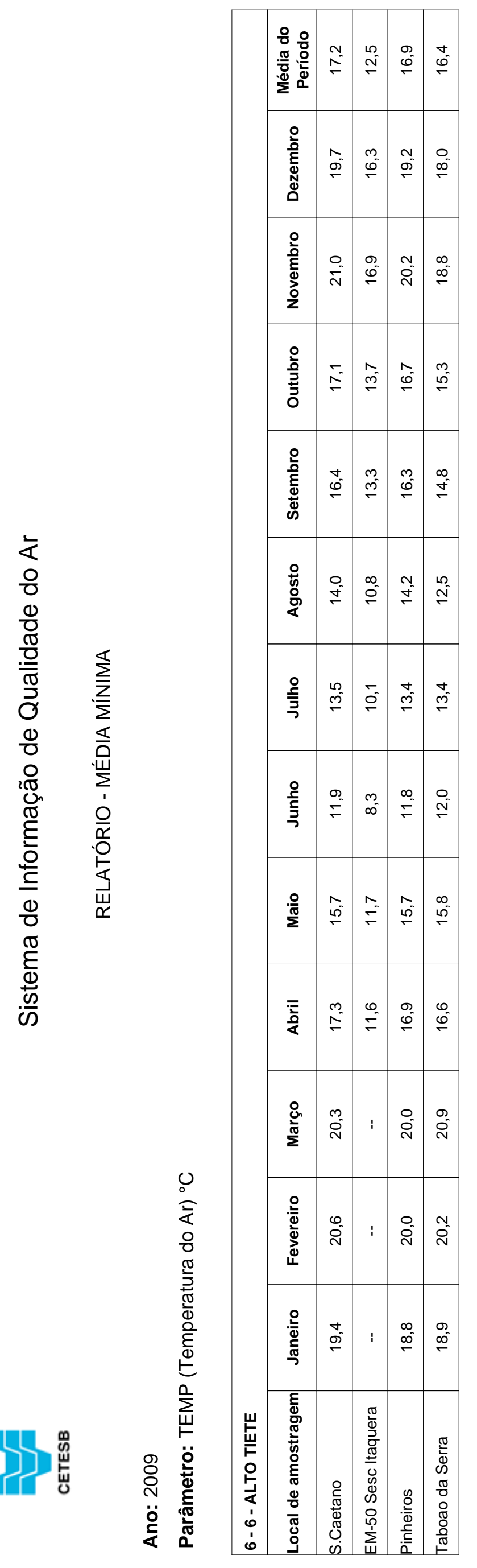




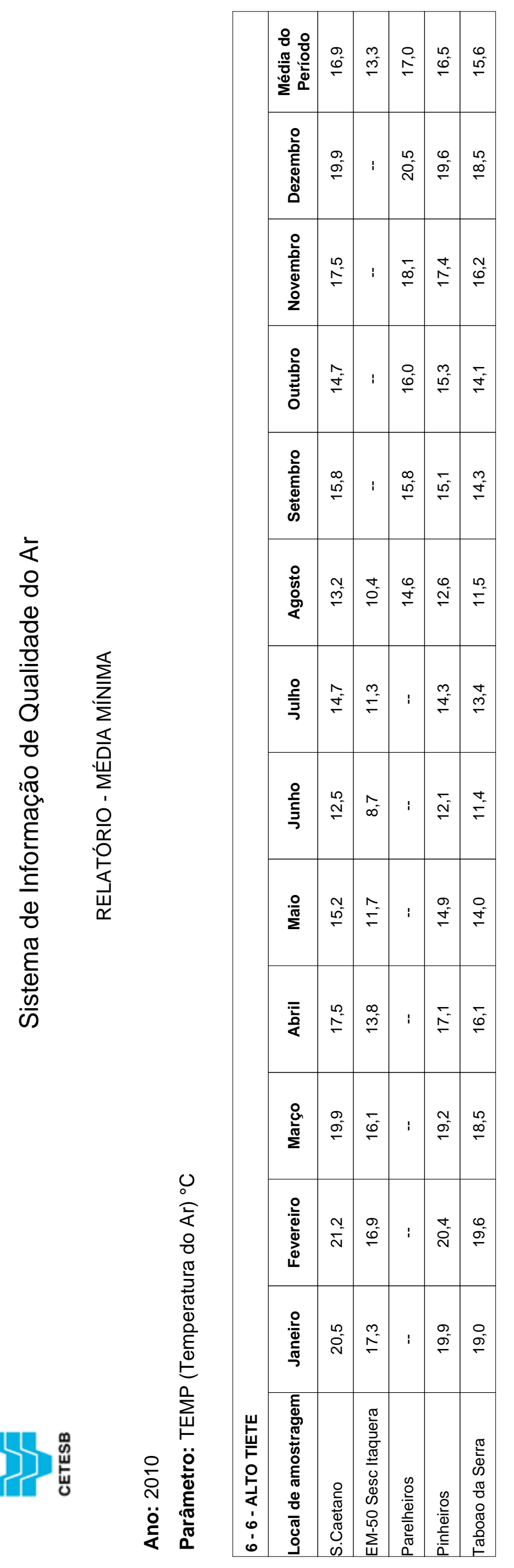




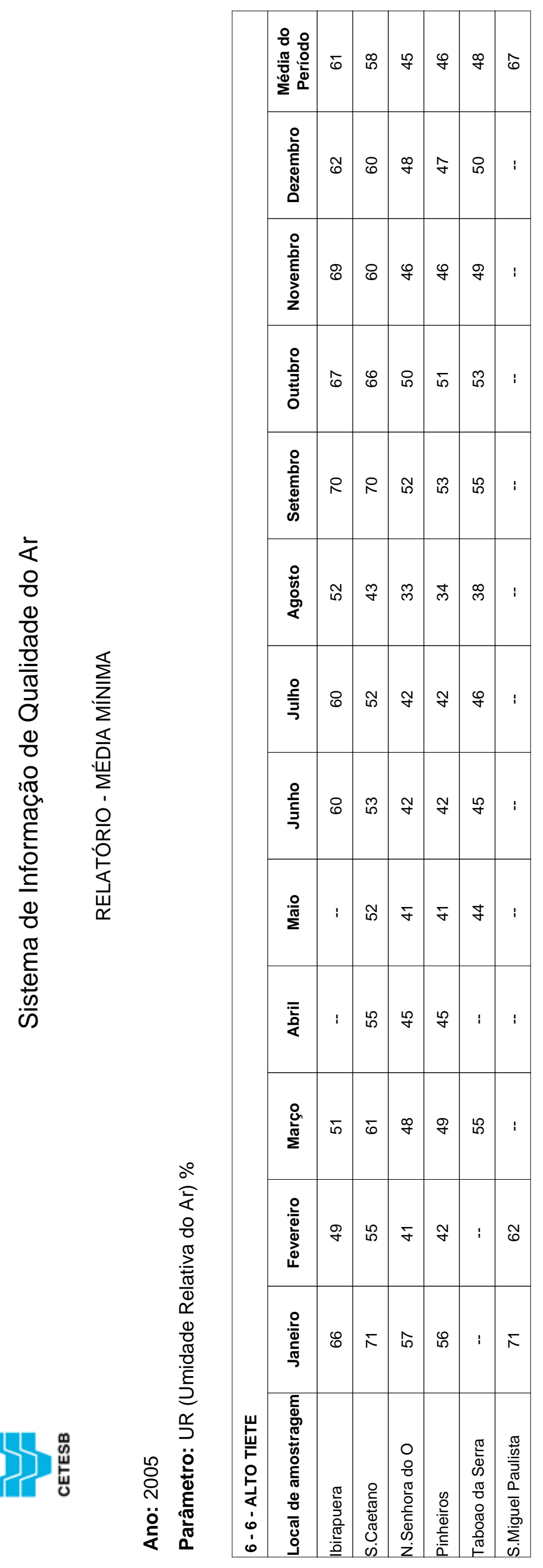




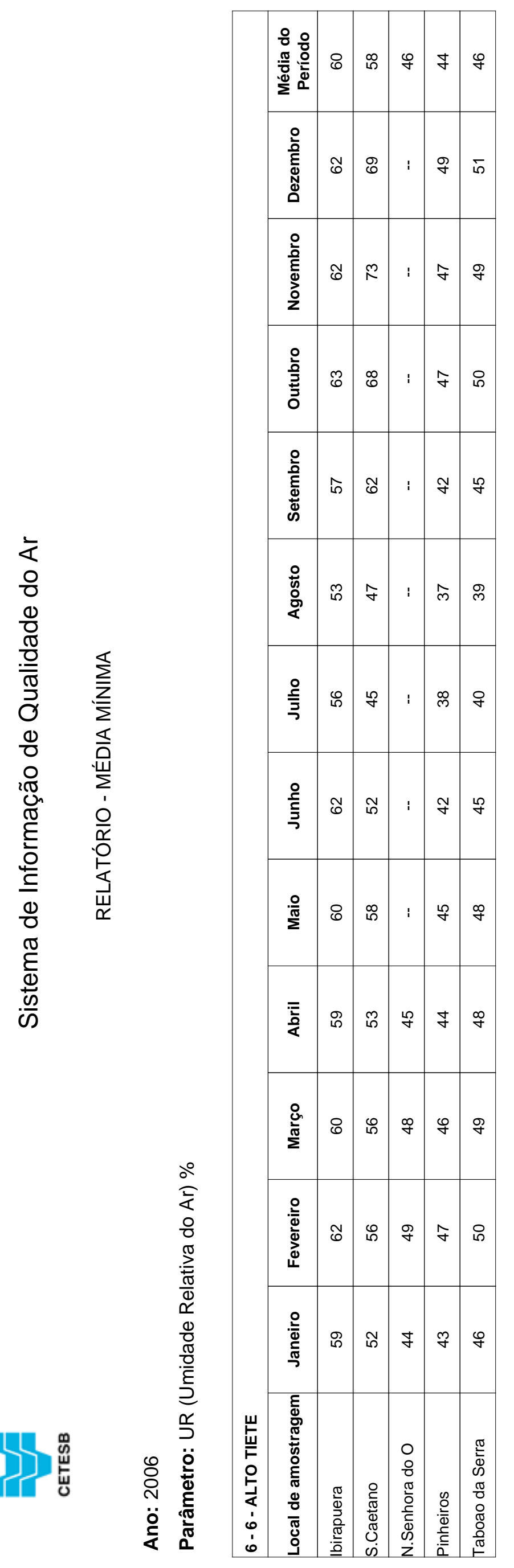




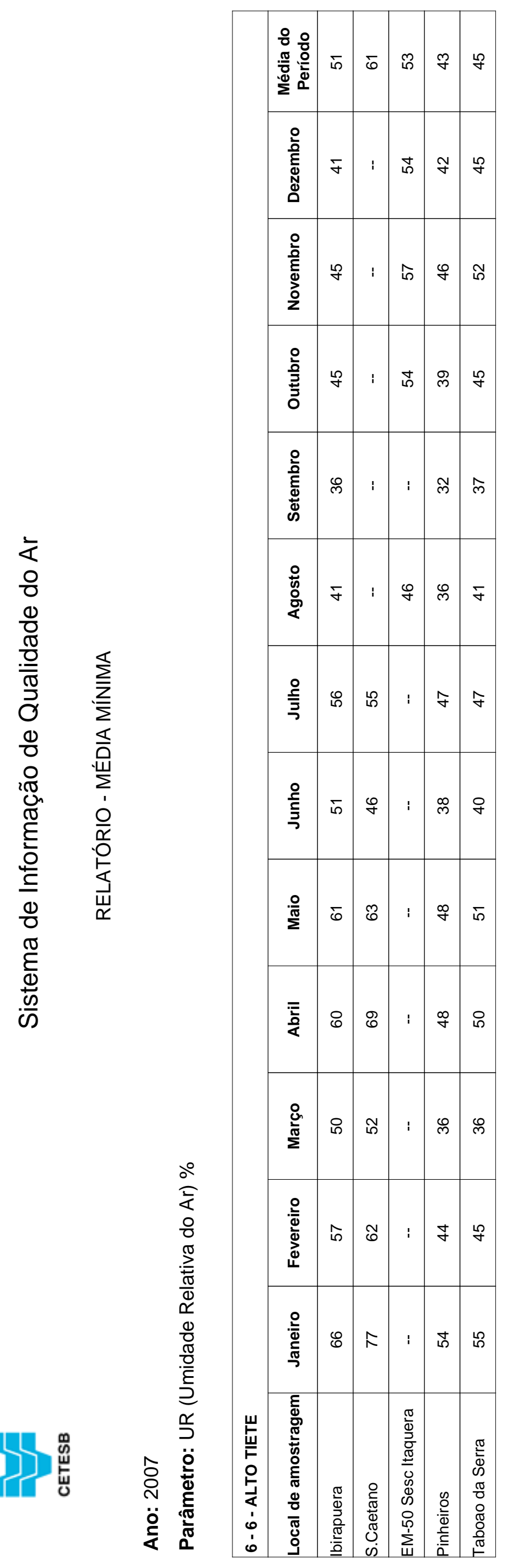




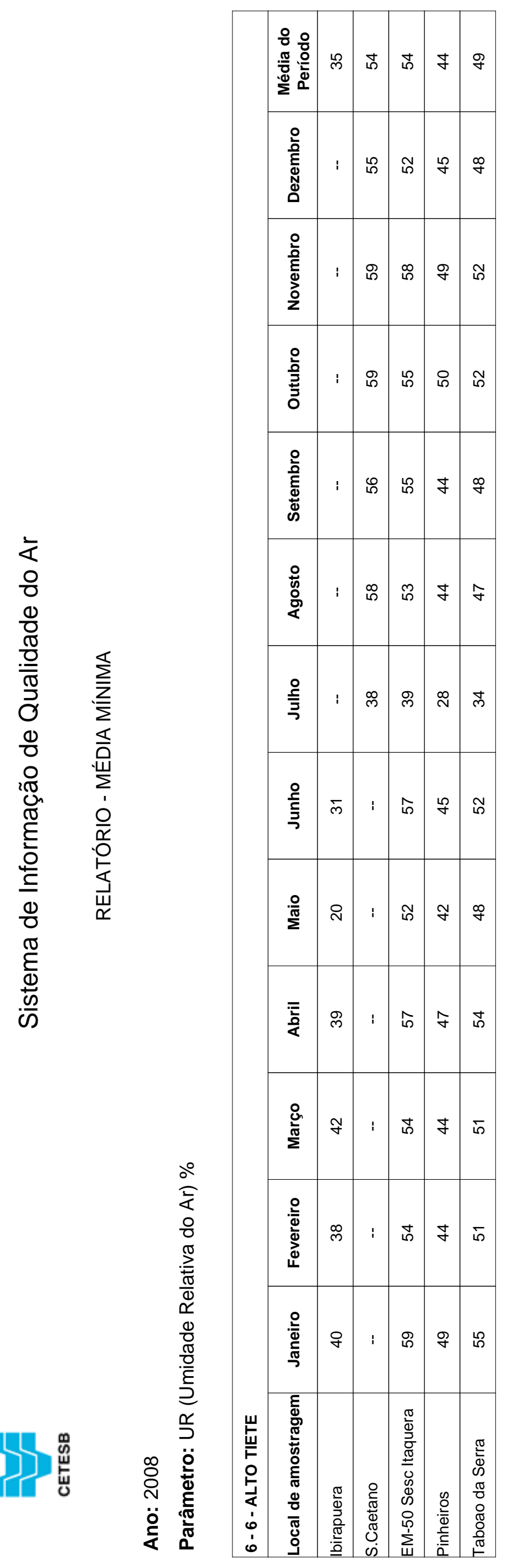




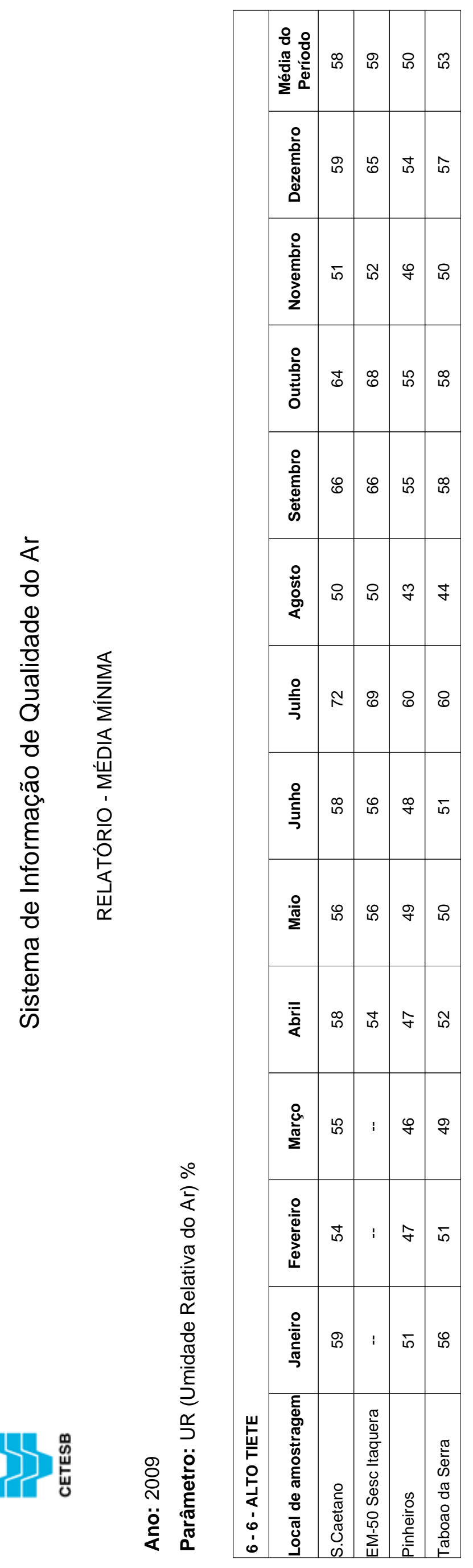




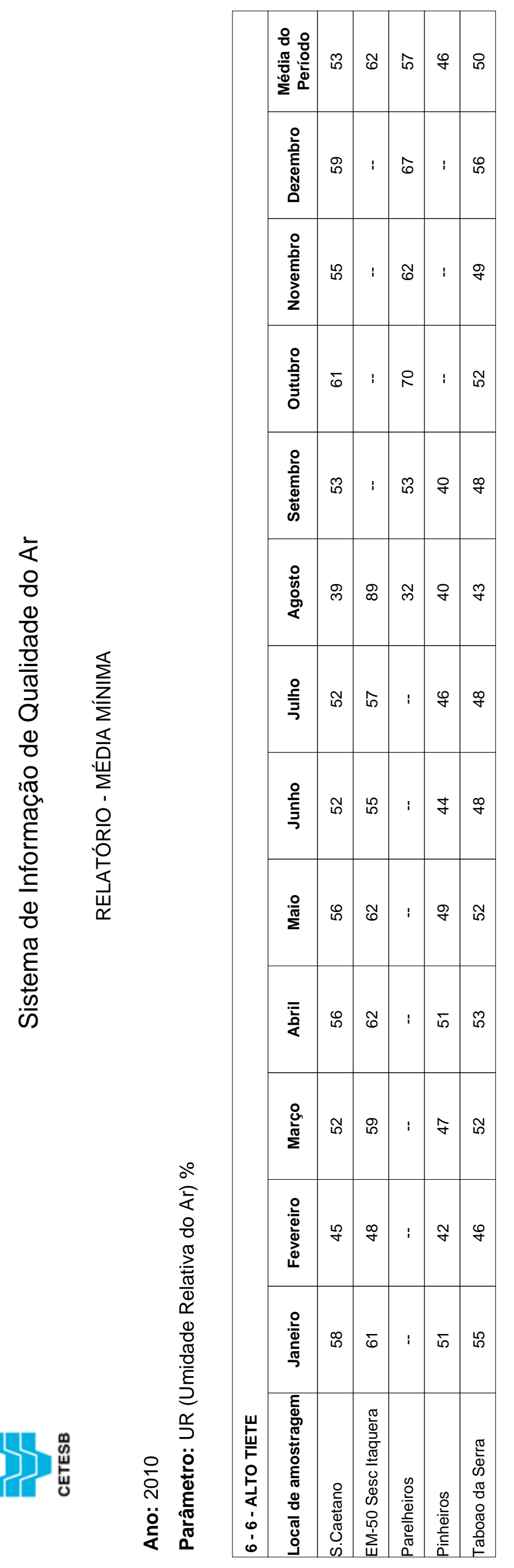

